\title{
Enantioselective Intermolecular [2+2] Photocycloaddition Reactions of 2(1H)-Quinolones Induced by Visible Light Irradiation
}

Andreas Tröster, Rafael Alonso, Andreas Bauer, and Thorsten Bach

Department Chemie and Catalysis Research Center (CRC), Technische Universität München, Lichtenbergstr. 4, D-85747 Garching

Table of Contents

1. General Information

2. Synthesis of Quinolones

3. Intermolecular [2+2] Photocycloaddition Reactions of Quinolones

4. Proof of Constitution and Configuration

5. Synthesis of the Thioxanthone Derivative 7

6. Competition Experiment

7. Emission Spectra of the Light Source

8. Solar Irradiation Experiment

9. NMR-Spectra of New Compounds

10. Representative HPLC/GC Traces 


\section{General Information}

All reactions sensitive to air or moisture were carried out in flame-dried glassware under argon pressure using standard Schlenk techniques. Dry tetrahydrofuran (THF) and dichloromethane $\left(\mathrm{CH}_{2} \mathrm{Cl}_{2}\right)$ were obtained from an MBraun MB-SPS 800 solvent purification system. Other dry solvents were obtained from Fluka and Acros in the highest purity available and used without further purification. Technical solvents used for aqueous workup and for column chromatography [n-pentane (pentane), ethyl acetate (EtOAc), methanol $(\mathrm{MeOH})$, dichloromethane $\left.\left(\mathrm{CH}_{2} \mathrm{Cl}_{2}\right)\right]$ were distilled prior to use. Photochemical experiments were performed in pyrex tubes $(\varnothing=1.0 \mathrm{~cm}$ for racemic reactions, $\varnothing=0.8 \mathrm{~cm}$ for enantioselective reactions and $\varnothing=1.8 \mathrm{~cm}$ for $0.15 \mathrm{mmol}$ scale reactions) in Rayonet type photochemical reactors equipped with 16 lamps $[\lambda=419 \mathrm{~nm}$ (RPR-4190 ̊, Rayonet)]. Prior to irradiation, the reaction mixture was degassed by purging with argon in an ultrasonicating bath for 15 minutes. Flash chromatography was performed on silica 60 (Merck, 230-400 mesh) with the indicated eluent mixtures. Thin layer chromatography (TLC) was performed on silica coated glass plates (silica $60 \mathrm{~F} 254)$ with detection by UV $(\lambda=254$ and $366 \mathrm{~nm})$. HPLC analyses were performed using a chiral stationary phase [ChiralPak AD-H (250 x $4.6 \mathrm{~mm})$, ChiralCell OD-H (250 x $4.6 \mathrm{~mm})$, Chiralcel, OJ-H, (250 x 4.6), Chiralpak AS-H (250 x $4.6 \mathrm{~mm}$ ), Chiralpak AS-RH, $150 \times 4.6 \mathrm{~mm}$ ) Daicel Chemical Industries] with UVD 340 Photodiode Array Detector, P580 Pump and an ASI-100 Automated Sample Injector at $20{ }^{\circ} \mathrm{C}$. Analytical gaschromatography was performed at a HP 6890 Series GC (Agilent, achiral stationary phase: HP-5 column, poly-dimethyl/diphenyl-siloxane, 95/5; chiral stationary phase: 2,3-dimethyl-6-TBDMS- $\beta$-cyclodextrine modified column) with a flame ionisation detector. The temperature method is given for the corresponding compounds. IR spectra were recorded on a JASCO IR-4100 (ATR), MS/HRMS measurements were performed on a Thermo Scientific DFS HRMS high resolution magnetic sector mass spectrometer. ${ }^{1} \mathrm{H}$ and ${ }^{13} \mathrm{C}$-NMR-spectra were recorded at $303 \mathrm{~K}$ either on a Bruker AVHD400 or a Bruker AVHD500 spectrometer. NMR spectra were calibrated to the respective residual solvent signals of $\mathrm{CDCl}_{3} \delta\left({ }^{1} \mathrm{H}\right)=7.26 \mathrm{ppm}, \delta\left({ }^{13} \mathrm{C}\right)$ $=77.16 \mathrm{ppm}\left(\mathrm{DMSO} \delta\left({ }^{1} \mathrm{H}\right)=2.50 \mathrm{ppm}\right)$. Apparent multiplets which occur as a result of accidental equality of coupling constants to those of magnetically non-equivalent protons are marked as virtual (virt.). The relative configuration of chiral products and the multiplicity of the ${ }^{13} \mathrm{C}$ NMR signals were determined by two-dimensional NMR spectra (COSY, HSQC, HMBC, NOESY). UV-Vis spectra were recorded on a PerkinElmer Lambda $35 \mathrm{UV}-\mathrm{V}$ is spectrometer. Melting points were measured on a Büchi 510 instrument and are not corrected. Specific rotations were determined with a ADP440+ Polarimeter. 


\section{Synthesis of Quinolones}

\section{5-Methylquinoline (S1)}

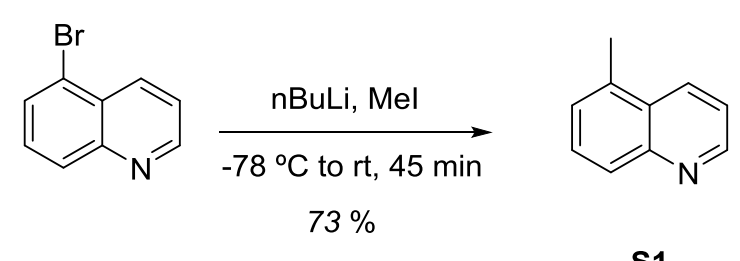

S1

A solution of 5-bromoquinoline (985 mg, $4.73 \mathrm{mmol}, 1.00 \mathrm{eq}$ ) in dry THF (47 mL) was cooled to $-78{ }^{\circ} \mathrm{C}$ under argon atmosphere and n-BuLi (2.5 $\mathrm{M}$ in hexane) $(2.46 \mathrm{ml}$, $6.15 \mathrm{mmol}, 1.30 \mathrm{eq})$ was added dropwise. After addition the solution was stirred at -78 ${ }^{\circ} \mathrm{C}$ for $15 \mathrm{~min}$. Iodomethane $(1.33 \mathrm{ml}, 21.30 \mathrm{mmol}, 4.5 \mathrm{eq})$ was added dropwise and the mixture was allowed to reach room temperature over $30 \mathrm{~min}$, quenched with a solution of ammonium choride and extracted with diethyl ether. The organic phase was dried with $\mathrm{Na}_{2} \mathrm{SO}_{4}$ and the solvent was removed under reduced pressure. The crude product was purified by column chromatography (silica, pentane/ethyl acetate 4:1). Quinoline S1 (497 mg, $3.47 \mathrm{mmol}, 73 \%$ ) was obtained as a colorless solid. The analytical data are in agreement with the literature. ${ }^{[R S C A d v .2014,4,21456-21464 .]}$

${ }^{1} \mathbf{H}-\mathbf{N M R}\left(500 \mathrm{MHz}, \mathrm{CDCl}_{3}, 303 \mathrm{~K}\right): \delta(\mathrm{ppm})=8.91\left(\mathrm{dd},{ }^{3} \mathrm{~J}=4.2 \mathrm{~Hz},{ }^{4} \mathrm{~J}=1.6 \mathrm{~Hz}, 1 \mathrm{H}\right)$, 8.32 (virt. td, $\left.{ }^{3} J \approx{ }^{3} J=8.5 \mathrm{~Hz},{ }^{4} J=1.6 \mathrm{~Hz}, 1 \mathrm{H}\right), 7.97\left(\mathrm{~d},{ }^{3} J=8.5 \mathrm{~Hz}, 1 \mathrm{H}\right), 7.64-7.57$ (m, 1H), $7.42\left(\mathrm{dd},{ }^{3} J=8.5 \mathrm{~Hz},{ }^{3} J=4.2 \mathrm{~Hz}, 1 \mathrm{H}\right), 7.37\left(\mathrm{~d},{ }^{3} J=7.1 \mathrm{~Hz}, 1 \mathrm{H}\right), 2.68(\mathrm{~s}, 3 \mathrm{H})$.

${ }^{13}$ C-NMR $\left(100 \mathrm{MHz}, \mathrm{CDCl}_{3}, 303 \mathrm{~K}\right): \delta(\mathrm{ppm})=150.0,148.6,134.7,132.7,129.3$, $127.8,127.8,127.2,120.8,18.7$.

\section{5-Methylquinoline 1-oxide (S2)}<smiles>Cc1cccc2ncccc12</smiles>

S1

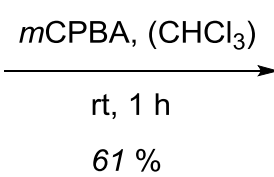

$61 \%$

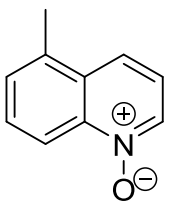

S2

A solution of 5-methylquinoline (S1) $(480 \mathrm{mg}, 3.35 \mathrm{mmol}, 1.00 \mathrm{eq})$ in $13 \mathrm{~mL}$ of chloroform was treated with meta-chloroperbenzoic acid (1.164 g, $5.20 \mathrm{mmol}, 1.55 \mathrm{eq})$. The mixture was stirred for $1 \mathrm{~h}$ at room temperature. Subsequently, saturated $\mathrm{NaHCO}_{3}$ $(17 \mathrm{~mL})$ and $2 \mathrm{M} \mathrm{NaOH}(17 \mathrm{~mL})$ were added and the mixture was extracted with dichloromethane. The combined organic layers were dried with $\mathrm{Na}_{2} \mathrm{SO}_{4}$ and the solvent was removed at reduced pressure yielding the desired N-oxide S2 (324 mg, $2.04 \mathrm{mmol}$, $61 \%)$ as a bright yellow solid which was used in the following step without further purification. The analytical data are in agreement with the literature. ${ }^{[J .}$ Chem. Soc. B, 1970, 440-443.]

${ }^{1} \mathbf{H}-\mathbf{N M R}\left(500 \mathrm{MHz}, \mathrm{CDCl}_{3}, 303 \mathrm{~K}\right): \delta(\mathrm{ppm})=8.74\left(\right.$ virt. $\mathrm{td},{ }^{3} \mathrm{~J} \approx{ }^{3} \mathrm{~J}=8.9 \mathrm{~Hz},{ }^{4} \mathrm{~J}=1.0$ $\mathrm{Hz}, 1 \mathrm{H}), 8.56\left(\mathrm{dd},{ }^{3} \mathrm{~J}=6.0 \mathrm{~Hz},{ }^{4} \mathrm{~J}=1.0 \mathrm{~Hz}, 1 \mathrm{H}\right), 8.09\left(\mathrm{dd},{ }^{3} \mathrm{~J}=8.8 \mathrm{~Hz},{ }^{4} \mathrm{~J}=1.0 \mathrm{~Hz}, 1 \mathrm{H}\right)$, 
$7.91\left(\mathrm{dd},{ }^{3} J=7.5 \mathrm{~Hz},{ }^{4} J=1.0 \mathrm{~Hz}, 1 \mathrm{H}\right), 7.59\left(\mathrm{dd},{ }^{3} J=8.8 \mathrm{~Hz},{ }^{3} J=7.5 \mathrm{~Hz}, 1 \mathrm{H}\right), 7.39$ (dd, $\left.{ }^{3} J=8.8 \mathrm{~Hz},{ }^{3} \mathrm{~J}=6.0 \mathrm{~Hz}, 1 \mathrm{H}\right), 2.72(\mathrm{~s}, 3 \mathrm{H})$.

${ }^{13} \mathrm{C}-\mathrm{NMR}\left(100 \mathrm{MHz}, \mathrm{CDCl}_{3}, 303 \mathrm{~K}\right): \delta(\mathrm{ppm})=142.0,135.5,135.4,130.1,130.0$, 129.3, 122.9, 120.4, 117.9, 19.1 .

\section{5-Methylquinolin-2-(1H)-one (S3)}<smiles>Cc1cccc2c1ccc[n+]2[O-]</smiles>

S2

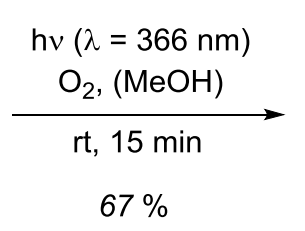<smiles>Cc1cccc2[nH]c(=O)ccc12</smiles>

5-Methylquinoline 1-oxide (S2) (90 mg, $0.57 \mathrm{mmol}$ ) was disolved in methanol (HPLC grade, $80 \mathrm{~mL}$ ). The solution was then saturated with oxygen, transferred into 7 phototubes and irradiated at $\lambda=366 \mathrm{~nm}$ in a Rayonet photoreactor at room temperature for $15 \mathrm{~min}$. Subsequently the solvent was removed under reduced pressure and the crude product was purified by column chromatography (silica, pentane/ethyl acetate 1:1). Quinolone S3 (60.4 mg, $0.38 \mathrm{mmol}, 67 \%$ ) was isolated as a colorless solid. The analytical data are in agreement with the literature. ${ }^{[P L O S O N E 2015,10, \text { e0113705] }}$

${ }^{1} \mathbf{H}$-NMR $\left(500 \mathrm{MHz}, \mathrm{CDCl}_{3}, 303 \mathrm{~K}\right): \delta$ (ppm) $=12.55$ (br. s, $\left.1 \mathrm{H}\right), 8.05\left(\mathrm{~d},{ }^{3} \mathrm{~J}=9.7 \mathrm{~Hz}\right.$, $1 \mathrm{H}), 7.41\left(\mathrm{dd},{ }^{3} \mathrm{~J}=8.2 \mathrm{~Hz},{ }^{3} \mathrm{~J}=7.2 \mathrm{~Hz}, 1 \mathrm{H}\right), 7.33\left(\mathrm{~d},{ }^{3} J=8.2 \mathrm{~Hz}, 1 \mathrm{H}\right), 7.06\left(\mathrm{~d},{ }^{3} J=7.2\right.$ $\mathrm{Hz}, 1 \mathrm{H}), 6.76\left(\mathrm{~d},{ }^{3} \mathrm{~J}=9.7 \mathrm{~Hz}, 1 \mathrm{H}\right), 2.64(\mathrm{~s}, 3 \mathrm{H})$.

${ }^{13}$ C-NMR $\left(400 \mathrm{MHz}, \mathrm{CDCl}_{3}, 303 \mathrm{~K}\right): \delta(\mathrm{ppm})=164.4,138.9,138.0,135.6,130.8$, 124.2, 120.7, 119.0, 114.7, 18.8 .

\section{6-Bromo-2-chloroquinoline (S4)}

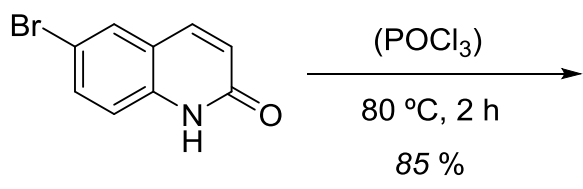<smiles>Clc1ccc2cc(Br)ccc2n1</smiles>

6-Bromoquinolin-2(1H)-one (700 mg, $3.12 \mathrm{mmol}, 1.00 \mathrm{eq}$ ) was suspended in $\mathrm{POCl}_{3}$ $(0.5 \mathrm{~mL}, 5.38 \mathrm{mmol}, 1.72 \mathrm{eq})$ under inert atmosphere. The suspension was heated at $80{ }^{\circ} \mathrm{C}$ for 2 hours. After the mixture was cooled at room temperature, the dark red thick suspension was poured onto an ice-water mixture. The mixture was then rendered basic with $8 \mathrm{M} \mathrm{NaOH}$ and extracted with dichloromethane. The organic layers were washed with water, brine, dried over $\mathrm{Na}_{2} \mathrm{SO}_{4}$ and concentrated in vacuo. The crude product was then purified by column chromatography (silica, pentane/ethyl acetate 9:1). Quinoline S4 (643 mg, $2.65 \mathrm{mmol}, 85 \%$ ) was isolated as a colorless solid. The analytical data are in agreement with the literature. ${ }^{\text {[Tetrahedron Lett. 2014, 55, 7130-7132.] }}$

${ }^{1} \mathbf{H}$-NMR $\left(500 \mathrm{MHz}, \mathrm{CDCl}_{3}, 303 \mathrm{~K}\right): \delta(\mathrm{ppm})=8.03\left(\mathrm{~d},{ }^{3} \mathrm{~J}=8.6 \mathrm{~Hz}, 1 \mathrm{H}\right), 7.99\left(\mathrm{~d},{ }^{4} J=\right.$ $2.1 \mathrm{~Hz}, 1 \mathrm{H}), 7.90\left(\mathrm{~d},{ }^{3} J=9.0 \mathrm{~Hz}, 1 \mathrm{H}\right), 7.81\left(\mathrm{dd},{ }^{3} J=9.0 \mathrm{~Hz},{ }^{4} J=2.1 \mathrm{~Hz}, 1 \mathrm{H}\right), 7.42(\mathrm{~d}$, $\left.{ }^{3} J=8.6 \mathrm{~Hz}, 1 \mathrm{H}\right)$. 


\section{6-Methyl-2-chloroquinoline (S5)}

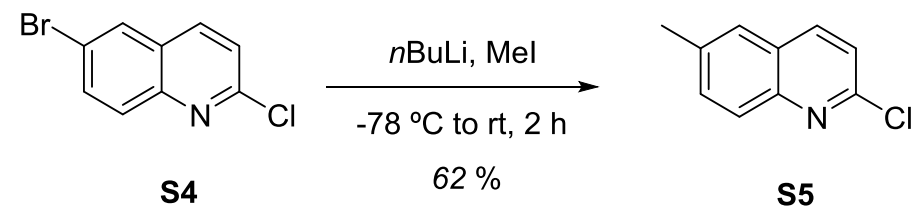

A solution of 6-bromo-2-chloroquinoline (S4) (300 mg, $1.24 \mathrm{mmol}, 1.00 \mathrm{eq})$ in dry THF $(10 \mathrm{~mL})$ was cooled to $-78{ }^{\circ} \mathrm{C}$ under argon atmosphere and $\mathrm{n}$-BuLi (2.5 $\mathrm{M}$ in hexane) (0.61 ml, $1.51 \mathrm{mmol}, 1.22 \mathrm{eq})$ was added dropwise. After addition the solution was stirred at $-78{ }^{\circ} \mathrm{C}$ for $1 \mathrm{~h}$. Iodomethane $(0.08 \mathrm{ml}, 1.24 \mathrm{mmol}, 1.00 \mathrm{eq})$ was added dropwise and the mixture was stirred at this temperature for $1 \mathrm{~h}$, then it was quenched with water at $-20{ }^{\circ} \mathrm{C}$, extracted with ethyl acetate, dried with $\mathrm{Na}_{2} \mathrm{SO}_{4}$ and the solvent was removed under reduced pressure. The crude product was purified by column chromatography (silica, pentane/ethyl acetate 4:1). Quinoline S5 (137 mg, $0.77 \mathrm{mmol}$, $62 \%)$ was obtained as a colorless solid. The analytical data are in agreement with the literature. [ Tetrahedron Lett. 2014, 55, 7130-7132]

${ }^{1} \mathbf{H}$-NMR $\left(500 \mathrm{MHz}, \mathrm{CDCl}_{3}, 303 \mathrm{~K}\right): \delta(\mathrm{ppm})=7.99\left(\mathrm{~d},{ }^{3} \mathrm{~J}=8.7 \mathrm{~Hz}, 1 \mathrm{H}\right), 7.91\left(\mathrm{~d},{ }^{3} J=\right.$ $9.1 \mathrm{~Hz}, 1 \mathrm{H}), 7.61-7.53(\mathrm{~m}, 2 \mathrm{H}), 7.33\left(\mathrm{~d},{ }^{3} \mathrm{~J}=8.7 \mathrm{~Hz}, 1 \mathrm{H}\right), 2.53$ (s, 3H).

\section{6-Methylquinolin-2-(1H)-one (S6)}

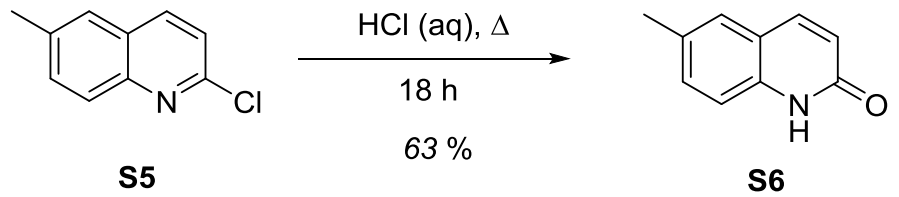

6-Methyl-2-chloroquinoline (75 mg, $0.42 \mathrm{mmol}, 1.00 \mathrm{eq}$ ) was refluxed in $\mathrm{HCl} 6 \mathrm{M}$ $(1.5 \mathrm{~mL})$ for $22 \mathrm{~h}$. The reaction mixture was cooled to room temperature, and it was extracted with dichloromethane and dried with $\mathrm{Na}_{2} \mathrm{SO}_{4}$. The crude residue was purified by column chromatography (silica, pentane/ethyl acetate 1:1). 6-Methylquinolin-2(1H)-one (S6) (42.1 mg, $0.26 \mathrm{mmol}, 63 \%$ ) was obtained as a colorless solid. The analytical data are in agreement with the literature. ${ }^{[O r g}$. Lett. 2015, 17, 222-225.]

${ }^{1} \mathbf{H}$-NMR (500 MHz, $\left.\mathrm{CDCl}_{3}, 303 \mathrm{~K}\right): \delta(\mathrm{ppm})=12.44$ (br. s, $\left.1 \mathrm{H}\right), 7.77\left(\mathrm{~d},{ }^{3} \mathrm{~J}=9.4 \mathrm{~Hz}\right.$, $1 \mathrm{H}), 7.41-7.30(\mathrm{~m}, 3 \mathrm{H}), 6.71\left(\mathrm{~d},{ }^{3} J=9.4 \mathrm{~Hz}, 1 \mathrm{H}\right), 2.41(\mathrm{~s}, 3 \mathrm{H})$.

${ }^{13}$ C-NMR $\left(100 \mathrm{MHz}, \mathrm{CDCl}_{3}, 303 \mathrm{~K}\right): \delta(\mathrm{ppm})=163.9,141.0,136.6,132.5,132.3$, 127.5, 121.3, 120.1, 116.2, 21.0. 
7-Methylquinoline 1-oxide (S7)

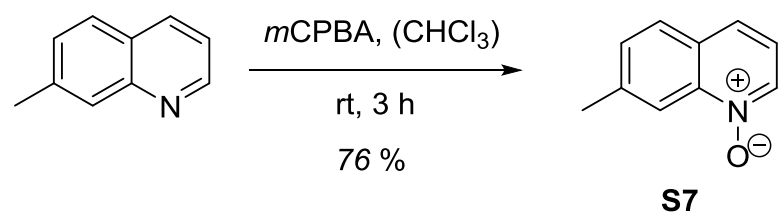

A solution of 7-methylquinoline $(1.00 \mathrm{~g}, 6.98 \mathrm{mmol}, 1.00 \mathrm{eq})$ in $20 \mathrm{~mL}$ of chloroform was treated with meta-chloroperbenzoic acid $(2.67 \mathrm{~g}, 7.57 \mathrm{mmol}, 1.55 \mathrm{eq})$. The mixture was stirred for $3 \mathrm{~h}$ at room temperature. Subsequently saturated $\mathrm{NaHCO}_{3}(25 \mathrm{~mL})$ and $2 \mathrm{M} \mathrm{NaOH}(25 \mathrm{~mL})$ were added and the mixture was extracted with dichloromethane. The combined organic layers were dried with $\mathrm{Na}_{2} \mathrm{SO}_{4}$ and the solvent was removed at reduced pressure yielding the desired N-oxide $\mathbf{S} 7(844 \mathrm{mg}, 5.30 \mathrm{mmol}, 76 \%$ ) as a yellow solid which was used in the following step without further purification. The

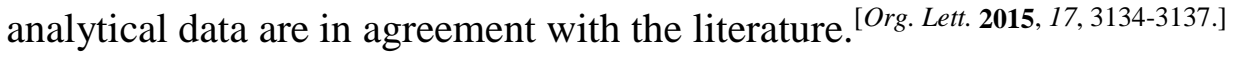

${ }^{1} \mathbf{H}-\mathbf{N M R}\left(500 \mathrm{MHz}, \mathrm{CDCl}_{3}, 303 \mathrm{~K}\right): \delta(\mathrm{ppm})=8.54(\mathrm{~s}, 1 \mathrm{H}), 8.51\left(\mathrm{~d},{ }^{3} J=5.9 \mathrm{~Hz}, 1 \mathrm{H}\right)$, $7.75\left(\mathrm{~d},{ }^{3} J=8.4 \mathrm{~Hz}, 1 \mathrm{H}\right), 7.70\left(\mathrm{~d},{ }^{3} J=8.4 \mathrm{~Hz}, 1 \mathrm{H}\right), 7.46\left(\mathrm{~d},{ }^{3} J=8.4 \mathrm{~Hz}, 1 \mathrm{H}\right), 7.22(\mathrm{dd}$, $\left.{ }^{3} J=8.4 \mathrm{~Hz},{ }^{3} J=5.9 \mathrm{~Hz}, 1 \mathrm{H}\right), 2.58(\mathrm{~s}, 3 \mathrm{H})$.

${ }^{13}$ C-NMR $\left(100 \mathrm{MHz}, \mathrm{CDCl}_{3}, 303 \mathrm{~K}\right): \delta(\mathrm{ppm})=141.8,141.6,135.9,131.1,128.8$, 128.0, 126.2, 120.1, 118.9, 22.2 .

\section{7-Methylquinolin-2-(1H)-one (S8)}
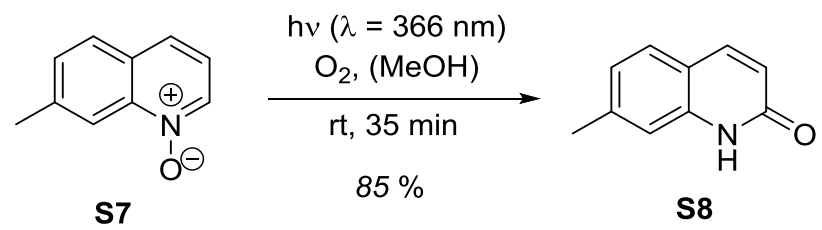

7-Methylquinoline 1-oxide (S7) (250 mg, $1.57 \mathrm{mmol}$ ) was disolved in methanol (HPLC grade, $50 \mathrm{~mL}$ ). The solution was then saturated with oxygen, transferred into 5 phototubes and irradiated at $\lambda=366 \mathrm{~nm}$ in a Rayonet photoreactor at room temperature for $35 \mathrm{~min}$. Subsequently the solvent was removed under reduced pressure and the crude product was then purified by column chromatography (silica, pentane/ethyl acetate 1:1). Quinolone S8 (212 $\mathrm{mg}, 1.33 \mathrm{mmol}, 85 \%)$ was isolated as a

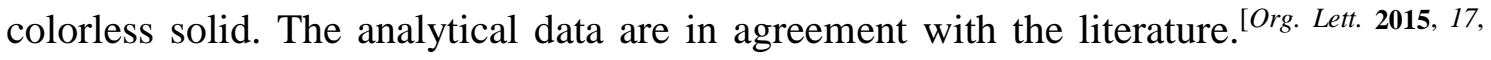
222-225.]

${ }^{1} \mathbf{H}$-NMR $\left(500 \mathrm{MHz}, \mathrm{CDCl}_{3}, 303 \mathrm{~K}\right): \delta(\mathrm{ppm})=12.63$ (br. s, $\left.1 \mathrm{H}\right), 7.80\left(\mathrm{~d},{ }^{3} \mathrm{~J}=9.4 \mathrm{~Hz}\right.$, $1 \mathrm{H}), 7.45\left(\mathrm{~d},{ }^{3} J=7.8 \mathrm{~Hz}, 1 \mathrm{H}\right), 7.27(\mathrm{~s}, 1 \mathrm{H}), 7.05\left(\mathrm{~d},{ }^{3} J=7.8 \mathrm{~Hz}, 1 \mathrm{H}\right), 6.68\left(\mathrm{~d},{ }^{3} J=9.4\right.$ $\mathrm{Hz}, 1 \mathrm{H}), 2.46$ (s, 3H).

${ }^{13}$ C-NMR $\left(100 \mathrm{MHz}, \mathrm{CDCl}_{3}, 303 \mathrm{~K}\right): \delta(\mathrm{ppm})=165.0,141.8,141.2,138.8,127.6$, 124.6, 120.1, 118.1, 116.3, 21.9. 
8-Methylquinoline 1-oxide (S9)

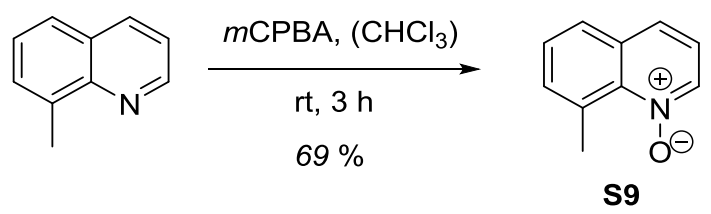

A solution of 8-methylquinoline $(1.00 \mathrm{~g}, 6.98 \mathrm{mmol}, 1.00 \mathrm{eq})$ in $20 \mathrm{~mL}$ of chloroform was treated with meta-chloroperbenzoic acid $(2.67 \mathrm{~g}, 7.57 \mathrm{mmol}, 1.55 \mathrm{eq})$. The mixture was stirred for $3 \mathrm{~h}$ at room temperature. Subsequently saturated $\mathrm{NaHCO}_{3}(25 \mathrm{~mL})$ and $2 \mathrm{M} \mathrm{NaOH}(25 \mathrm{~mL})$ were added and the mixture was extracted with dichloromethane. The combined organic layers were dried with $\mathrm{Na}_{2} \mathrm{SO}_{4}$ and the solvent was removed at reduced pressure yielding the desired N-oxide $\mathbf{S 9}$ (767 $\mathrm{mg}, 4.81 \mathrm{mmol}, 69 \%$ ) as a yellow solid which was used in the following step without further purification. The analytical data are in agreement with the literature. ${ }^{\text {[Org. Lett. 2015, 17, 3134-3137.] }}$

${ }^{1} \mathbf{H}-\mathbf{N M R}\left(500 \mathrm{MHz}, \mathrm{CDCl}_{3}, 303 \mathrm{~K}\right): \delta(\mathrm{ppm})=8.37\left(\mathrm{~d},{ }^{3} \mathrm{~J}=6.0 \mathrm{~Hz}, 1 \mathrm{H}\right), 7.71-7.53$ (m, 2H), $7.46-7.34(\mathrm{~m}, 2 \mathrm{H}), 7.16\left(\mathrm{dd},{ }^{3} J=7.0 \mathrm{~Hz},{ }^{3} J=5.2 \mathrm{~Hz}, 1 \mathrm{H}\right), 3.16(\mathrm{~s}, 3 \mathrm{H})$.

${ }^{13}$ C-NMR $\left(100 \mathrm{MHz}, \mathrm{CDCl}_{3}, 303 \mathrm{~K}\right): \delta(\mathrm{ppm})=141.5,137.4,133.7,133.5,132.6$, 128.2, 126.9, 126.6, 120.7, 25.0 .

8-Methylquinolin-2-(1H)-one (S10)<smiles>Cc1cccc2ccc[n+]([O-])c12</smiles>

S9<smiles>Cc1cccc2ccc(=O)[nH]c12</smiles>

S10

8-Methylquinoline 1-oxide (S9) (300 mg, $1.88 \mathrm{mmol}$ ) was disolved in methanol (HPLC grade, $60 \mathrm{~mL}$ ). The solution was then saturated with oxygen, transferred into 6 phototubes and irradiated at $\lambda=366 \mathrm{~nm}$ in a Rayonet photoreactor at room temperature for $35 \mathrm{~min}$. Subsequently the solvent was removed under reduced pressure and the crude product was purified by column chromatography (silica, pentane/ethyl acetate 1:1). Quinolone S10 (234 mg, $1.47 \mathrm{mmol}, 78 \%$ ) was isolated as a colorless solid. The analytical data are in agreement with the literature. [Org. Lett. 2015, 17, 222-225.]

${ }^{1} \mathbf{H}$-NMR $\left(500 \mathrm{MHz}, \mathrm{CDCl}_{3}, 303 \mathrm{~K}\right): \delta(\mathrm{ppm})=9.96$ (br. s, $\left.1 \mathrm{H}\right), 7.77$ (d, ${ }^{3} \mathrm{~J}=9.5 \mathrm{~Hz}$, $1 \mathrm{H}), 7.41\left(\mathrm{~d},{ }^{3} J=7.8 \mathrm{~Hz}, 1 \mathrm{H}\right), 7.35\left(\mathrm{~d},{ }^{3} J=7.3 \mathrm{~Hz}, 1 \mathrm{H}\right), 7.12$ (virt. t, ${ }^{3} J \approx{ }^{3} J=7.6 \mathrm{~Hz}$, $1 \mathrm{H}), 6.67\left(\mathrm{~d},{ }^{3} J=9.5 \mathrm{~Hz}, 1 \mathrm{H}\right), 2.52(\mathrm{~s}, 3 \mathrm{H})$.

${ }^{13}$ C-NMR $\left(100 \mathrm{MHz}, \mathrm{CDCl}_{3}, 303 \mathrm{~K}\right): \delta(\mathrm{ppm})=163.5,141.5,137.0,132.0,126.2$, 123.4, 122.5, 121.5, 119.9, 17.0 . 


\section{Intermolecular [2 + 2] Photocycloaddition Reactions of Quinolones}

Methyl $(1 R, 2 \mathrm{a} R, 8 \mathrm{~b} R)-3-0 x 0-1,2,2 \mathrm{a}, 3,4,8 \mathrm{~b}-\mathrm{hexahydrocyclobuta}[c]$ quinoline-1-carboxylate (4a) and Methyl (1S,2aR,8bR)-3-oxo-1,2,2a,3,4,8b-hexahydrocyclobuta[c]quinoline-1-carboxylate (S11)

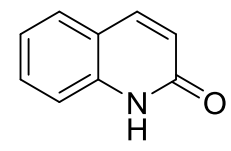<smiles>C=CC(=O)OC</smiles>

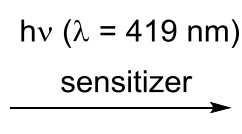

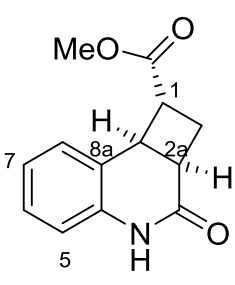

$4 a$<smiles>COC(=O)[C@H]1C[C@H]2C(=O)Nc3ccccc3[C@H]12</smiles>

S11

$\mathrm{C}_{13} \mathrm{H}_{13} \mathrm{NO}_{3}$ MW: $231,25 \mathrm{~g} / \mathrm{mol}$

\section{Racemic [2+2] Photocycloaddition}

2(1H)-Quinolone (20 mg, $0.14 \mathrm{mmol}, 1.00 \mathrm{eq}$.) and thioxanthen-9-one (15 mg, $0.07 \mathrm{mmol}, 0.50$ eq.) were dissolved in acetonitrile $(10 \mathrm{~mL}, c=14 \mathrm{mmol} / \mathrm{L})$. After degassing the solution, methyl acrylate ( $125 \mu \mathrm{l}, 1.4 \mathrm{mmol}, 10.00$ eq. $)$ was added and the mixture was irradiated at $419 \mathrm{~nm}$ for $5 \mathrm{~h}$ at room temperature. After removing the solvent, purification was achieved by flash column chromatography (silica, pentane/ethyl acetate 1:1). The title compounds $\mathbf{4 a}(22.4 \mathrm{mg}, 0.10 \mathrm{mmol}, 69 \%)$ and $\mathbf{S 1 1}$ (4.9 mg, $0.02 \mathrm{mmol}, 15 \%$ ) were obtained as colorless solids.

\section{Enantioselective [2+2] Photocycloaddition}

2(1H)-Quinolone (3.6 mg, $25.0 \mu \mathrm{mol}, 1.00$ eq.) and enantiomerically pure $3(1.1 \mathrm{mg}$, $2.5 \mu \mathrm{mol}, 10 \mathrm{~mol} \%$ ) were dissolved in $\alpha, \alpha, \alpha$-trifluorotoluene $(10 \mathrm{~mL}, c=2.5 \mathrm{mmol} / \mathrm{L})$. After degassing the solution, methyl acrylate (113 $\mu \mathrm{l}, 1.25 \mathrm{mmol}, 50.00$ eq.) was added and the mixture was irradiated at $419 \mathrm{~nm}$ for $6 \mathrm{~h}$ at $-25^{\circ} \mathrm{C}$. After removing the solvent, purification was achieved by flash column chromatography (silica, pentane/ethyl acetate 1:1). The title compound $4 \mathbf{a}(4.4 \mathrm{mg}, 19.0 \mu \mathrm{mol}, 76 \%, 81 \% e e)$ was obtained as a colorless solid.

Enantioselective [2+2] Photocycloaddition at $-40{ }^{\circ} \mathrm{C}$

2(1H)-Quinolone (3.6 mg, $25.0 \mu \mathrm{mol}, 1.00 \mathrm{eq}$.) and enantiomerically pure $3(1.1 \mathrm{mg}$, $2.5 \mu \mathrm{mol}, 10 \mathrm{~mol} \%)$ were dissolved in a solvent mixture [1,3-bis(trifluoromethyl)benzene and $\alpha, \alpha, \alpha$-trifluorotoluene $=2 / 1](10 \mathrm{~mL}, c=2.5 \mathrm{mmol} / \mathrm{L})$. After degassing the solution, methyl acrylate (113 $\mu \mathrm{l}, 1.25 \mathrm{mmol}, 50.00$ eq.) was added and the mixture was irradiated at $419 \mathrm{~nm}$ for $22 \mathrm{~h}$ at $-40{ }^{\circ} \mathrm{C}$. After removing the solvent, purification was achieved by flash column chromatography (silica, pentane/ethyl acetate 1:1). The title compound $\mathbf{4 a}(4.4 \mathrm{mg}, 19.0 \mu \mathrm{mol}, 85 \%, 83 \% e e)$ was obtained as a colorless solid. 
M.p.: $156-158^{\circ} \mathrm{C}$.

TLC: $R_{\mathrm{f}}=0.66$ (ethyl acetate, UV).

${ }^{1} \mathbf{H}$-NMR $\left(500 \mathrm{MHz}, \mathrm{CDCl}_{3}, 303 \mathrm{~K}\right): \delta(\mathrm{ppm})=9.36$ (br. s, $\left.1 \mathrm{H}, \mathrm{NH}\right), 7.17$ (virt. td, ${ }^{3} J \approx$ $\left.{ }^{3} J=7.7 \mathrm{~Hz},{ }^{4} J=1.6 \mathrm{~Hz}, 1 \mathrm{H}, \mathrm{H}-6\right), 7.10\left(\mathrm{dd},{ }^{3} J=7.5 \mathrm{~Hz},{ }^{4} J=1.6 \mathrm{~Hz}, 1 \mathrm{H}, \mathrm{H}-8\right), 6.98$ (virt. td, $\left.{ }^{3} J \approx{ }^{3} J=7.5 \mathrm{~Hz},{ }^{4} J=1.2 \mathrm{~Hz}, 1 \mathrm{H}, \mathrm{H}-7\right), 6.83\left(\mathrm{dd},{ }^{3} J=8.0 \mathrm{~Hz},{ }^{4} J=1.2 \mathrm{~Hz}, 1 \mathrm{H}\right.$, $\mathrm{H}-5), 4.02$ (virt. $\left.\mathrm{t},{ }^{3} \mathrm{~J} \approx{ }^{3} \mathrm{~J}=8.9 \mathrm{~Hz}, 1 \mathrm{H}, \mathrm{H}-8 \mathrm{~b}\right), 3.73(\mathrm{~s}, 3 \mathrm{H}, \mathrm{MeO}), 3.41-3.26(\mathrm{~m}, 2 \mathrm{H}$, $\mathrm{H}-1, \mathrm{H}-2 \mathrm{a}), 2.87$ (virt. dt, $\left.{ }^{2} J=12.1 \mathrm{~Hz},{ }^{3} J \approx{ }^{3} J=9.7 \mathrm{~Hz}, 1 \mathrm{H}, \mathrm{H}-2\right), 2.72-2.58(\mathrm{~m}, 1 \mathrm{H}$, $\mathrm{H}-2)$.

${ }^{1}$ H-NMR $(500 \mathrm{MHz}, \mathrm{DMSO}, 303 \mathrm{~K}): \delta(\mathrm{ppm})=10.18$ (br. s, $\left.1 \mathrm{H}, \mathrm{NH}\right), 7.14$ (virt. td, ${ }^{3} J$ $\left.\approx^{3} J=7.7 \mathrm{~Hz},{ }^{4} J=1.5 \mathrm{~Hz}, 1 \mathrm{H}, \mathrm{H}-6\right), 7.03\left(\mathrm{dd},{ }^{3} J=7.5 \mathrm{~Hz},{ }^{4} J=1.5 \mathrm{~Hz}, 1 \mathrm{H}, \mathrm{H}-8\right), 6.93-$ 6.85 (m, 2H, H-5, H-7), 3.86 (virt. $\left.\mathrm{t},{ }^{3} J \approx{ }^{3} J=9.0 \mathrm{~Hz}, 1 \mathrm{H}, \mathrm{H}-8 \mathrm{~b}\right), 3.64(\mathrm{~s}, 3 \mathrm{H}, \mathrm{MeO})$, 3.24 (virt. qd, ${ }^{3} J \approx{ }^{3} J \approx{ }^{3} J=9.1 \mathrm{~Hz},{ }^{4} J=1.3 \mathrm{~Hz}, 1 \mathrm{H}, \mathrm{H}-1$ ), 3.14 (virt. tdd, ${ }^{3} J \approx{ }^{3} J=9.8$ $\mathrm{Hz}, 3.0,{ }^{4} J=1.4 \mathrm{~Hz}, 1 \mathrm{H}, \mathrm{H}-2 \mathrm{a}$ ), 2.67 (virt. dt, ${ }^{2} J=11.8 \mathrm{~Hz},{ }^{3} J \approx{ }^{3} J=9.8 \mathrm{~Hz}, 1 \mathrm{H}, \mathrm{H}-2$ ), 2.35 (ddd, $\left.{ }^{2} J=11.8,{ }^{3} J=8.8 \mathrm{~Hz},{ }^{3} J=3.0 \mathrm{~Hz}, 1 \mathrm{H}, \mathrm{H}-2\right)$.

${ }^{13} \mathrm{C}-\mathrm{NMR}\left(100 \mathrm{MHz}, \mathrm{CDCl}_{3}, 303 \mathrm{~K}\right): \delta(\mathrm{ppm})=174.0$ (s, COOMe), 173.0 (s, C-3), 136.8 (s, C-4a), 128.5 (d, C-6), 128.0 (d, C-8), 123.8 (d, C-7), 122.5 (s, C-8a), 116.0 (d, C-5), 52.2 (q, OMe), 45.0 (d, C-2a), 40.1 (d, C-8b), 34.9 (d, C-1), 29.4 (t, C-2).

IR (ATR): $v\left(\mathrm{~cm}^{-1}\right)=1728,1671,1652,1592,1488,1383,1357,1304,1240,1201$, 1175, 1126, 1065, 1021, 942, 795, 766, 697, 669, 633, 620, 605.

MS (EI, $70 \mathrm{eV}): m / z(\%)=231(8)\left[\mathrm{M}^{+}\right], 216(0.4)\left[\mathrm{M}^{+}-\mathrm{CH}_{3}\right], 199(4)\left[\mathrm{M}^{+}-\mathrm{OCH}_{3}\right], 171$ (12) $\left[\mathrm{M}^{+}-\mathrm{C}_{2} \mathrm{H}_{3} \mathrm{O}_{2}\right], 145$ (100) $\left[\mathrm{M}^{+}-\mathrm{C}_{4} \mathrm{H}_{7} \mathrm{O}_{2}\right], 117$ (28), 90 (9).

HRMS (EI) $\left(\mathrm{C}_{13} \mathrm{H}_{13} \mathrm{NO}_{3}\right)$ : calc.: 231.0895; found: 231.0890 .

HPLC $\left(\right.$ AD-H, 250×4.6, $n$-hexane/iso-propanol = 90:10) $=\mathbf{4 a}, t_{\mathrm{R}}=15.2 \mathrm{~min}$; ent-4a, $16.8 \mathrm{~min})$.

\section{Endo isomer (S11):}

M.p.: $159-161^{\circ} \mathrm{C}$.

TLC: $R_{\mathrm{f}}=0.55$ (ethyl acetate, UV).

${ }^{1}$ H-NMR $\left(500 \mathrm{MHz}, \mathrm{CDCl}_{3}, 303 \mathrm{~K}\right): \delta(\mathrm{ppm})=7.96$ (br. s, $\left.1 \mathrm{H}, \mathrm{NH}\right), 7.14$ (virt. td, ${ }^{3} \boldsymbol{J} \approx$ $\left.{ }^{3} J=7.7 \mathrm{~Hz},{ }^{4} J=1.2 \mathrm{~Hz}, 1 \mathrm{H}, \mathrm{H}-6\right), 6.98\left(\mathrm{dd},{ }^{3} J=7.3 \mathrm{~Hz},{ }^{4} J=1.2 \mathrm{~Hz}, 1 \mathrm{H}, \mathrm{H}-8\right), 6.94$ (virt. td, $\left.{ }^{3} J \approx{ }^{3} J=7.4 \mathrm{~Hz},{ }^{4} J=1.2 \mathrm{~Hz}, 1 \mathrm{H}, \mathrm{H}-7\right), 6.68\left(\mathrm{dd},{ }^{3} J=7.9 \mathrm{~Hz},{ }^{4} J=1.2 \mathrm{~Hz}, 1 \mathrm{H}\right.$, $\mathrm{H}-5$ ), 4.25 (virt. $\mathrm{t},{ }^{3} J \approx{ }^{3} J=9.7 \mathrm{~Hz}, 1 \mathrm{H}, \mathrm{H}-8 \mathrm{~b}$ ), 3.64 (ddd, ${ }^{3} J=9.9 \mathrm{~Hz},{ }^{3} J=8.5 \mathrm{~Hz},{ }^{3} J=$ $7.2 \mathrm{~Hz}, 1 \mathrm{H}, \mathrm{H}-1$ ), 3.45 (virt. td, ${ }^{3} J \approx{ }^{3} J=9.9 \mathrm{~Hz},{ }^{3} J=7.0 \mathrm{~Hz}, 1 \mathrm{H}, \mathrm{H}-2 \mathrm{a}$ ), 3.39 (s, $3 \mathrm{H}$, $\mathrm{OMe}$ ), 2.89 (virt. dt, $\left.{ }^{2} J=12.6 \mathrm{~Hz},{ }^{3} J \approx{ }^{3} J=7.1 \mathrm{~Hz}, 1 \mathrm{H}, \mathrm{H}-2\right), 2.72\left(\mathrm{ddd},{ }^{2} J=12.6 \mathrm{~Hz},{ }^{3} J\right.$ $\left.=10.3 \mathrm{~Hz},{ }^{3} \mathrm{~J}=8.5 \mathrm{~Hz}, 1 \mathrm{H}, \mathrm{H}-2\right)$.

${ }^{13} \mathrm{C}$-NMR $\left(100 \mathrm{MHz}, \mathrm{CDCl}_{3}, 303 \mathrm{~K}\right): \delta(\mathrm{ppm})=172.3(\mathrm{~s}, \mathrm{COOMe}), 170.8(\mathrm{~s}, \mathrm{C}-3)$, 137.3 (s, C-4a), 129.0 (d, C-8), 128.6 (d, C-6), 123.3 (d, C-7), 119.5 (s, C-8a), 115.9 (d, C-5), 51.6 (q, OMe), 44.1 (d, C-1), 38.9 (d, C-8b), 34.9 (d, C-2a), 27.5 (t, C-2).

IR (ATR): $v\left(\mathrm{~cm}^{-1}\right)=1726,1669,1590,1492,1433,1400,1357,1323,1288,1241$, 1200, 1171, 1158, 1086, 1058, 1038, 938, 874, 842, 752, 731, 709, 679, 602.

MS (EI, $70 \mathrm{eV}): m / z(\%)=231(9)\left[\mathrm{M}^{+}\right], 216(0.2)\left[\mathrm{M}^{+}-\mathrm{CH}_{3}\right], 199(4)\left[\mathrm{M}^{+}-\mathrm{OCH}_{3}\right], 171$ (14) $\left[\mathrm{M}^{+}-\mathrm{C}_{2} \mathrm{H}_{3} \mathrm{O}_{2}\right], 145$ (100) $\left[\mathrm{M}^{+}-\mathrm{C}_{4} \mathrm{H}_{7} \mathrm{O}_{2}\right], 117$ (26), 90 (10).

HRMS (EI) $\left(\mathrm{C}_{13} \mathrm{H}_{13} \mathrm{NO}_{3}\right)$ : calc.: 231.0895; found: 231.0890 . 
<smiles>O=c1ccc2ccccc2[nH]1</smiles><smiles>C=CC(=O)OCC</smiles>

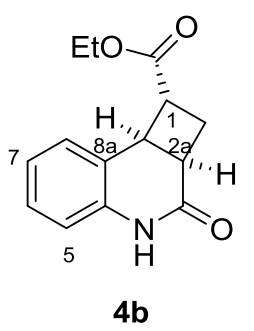<smiles>CCOC(=O)[C@H]1C[C@H]2C(=O)Nc3ccccc3[C@H]12</smiles>

S12

\section{Racemic [2+2] Photocycloaddition}

2(1H)-Quinolone (20 mg, $0.14 \mathrm{mmol}, 1.00$ eq.) and thioxanthen-9-one (15 mg, $0.07 \mathrm{mmol}, 0.50$ eq.) were dissolved in acetonitrile $(10 \mathrm{~mL}, c=14 \mathrm{mmol} / \mathrm{L})$. After degassing the solution, ethyl acrylate ( $150 \mu \mathrm{l}, 1.4 \mathrm{mmol}, 10.00$ eq.) was added and the mixture was irradiated at $419 \mathrm{~nm}$ for $5 \mathrm{~h}$ at room temperature. After removing the solvent, purification was achieved by flash column chromatography (silica, pentane/ethyl acetate 1:1). The title compounds $\mathbf{4 b}(22.0 \mathrm{mg}, 0.09 \mathrm{mmol}, 64 \%)$ and $\mathbf{S 1 2}$ (4.1 mg, $0.02 \mathrm{mmol}, 12 \%)$ were obtained as colorless solids.

\section{Enantioselective [2+2] Photocycloaddition}

2(1H)-Quinolone (3.6 mg, $25.0 \mu \mathrm{mol}, 1.00$ eq.) and enantiomerically pure $3(1.1 \mathrm{mg}$, $2.5 \mu \mathrm{mol}, 10 \mathrm{~mol} \%)$ were dissolved in $\alpha, \alpha, \alpha$-trifluorotoluene $(10 \mathrm{~mL}, c=2.5 \mathrm{mmol} / \mathrm{L})$. After degassing the solution, ethyl acrylate ( $138 \mu \mathrm{l}, 1.25 \mathrm{mmol}, 50.00$ eq.) was added and the mixture was irradiated at $419 \mathrm{~nm}$ for $6 \mathrm{~h}$ at $-25^{\circ} \mathrm{C}$. After removing the solvent, purification was achieved by flash column chromatography (silica, pentane/ethyl acetate 1:1). The title compound $\mathbf{4 b}(5.6 \mathrm{mg}, 23.0 \mu \mathrm{mol}, 82 \%, 78 \% e e)$ was obtained as a colorless solid.

Enantioselective [2+2] Photocycloaddition at $-40{ }^{\circ} \mathrm{C}$

2(1H)-Quinolone (3.6 mg, $25.0 \mu \mathrm{mol}, 1.00$ eq.) and enantiomerically pure $3(1.1 \mathrm{mg}$, $2.5 \mu \mathrm{mol}, \quad 10 \mathrm{~mol} \%)$ were dissolved in a solvent mixture [1,3-bis(trifluoromethyl)benzene and $\alpha, \alpha, \alpha$-trifluorotoluene $=2 / 1](10 \mathrm{~mL}, c=2.5 \mathrm{mmol} / \mathrm{L})$. After degassing the solution, ethyl acrylate (138 $\mu \mathrm{l}, 1.25 \mathrm{mmol}, 50.00$ eq. $)$ was added and the mixture was irradiated at $419 \mathrm{~nm}$ for $45 \mathrm{~h}$ at $-40{ }^{\circ} \mathrm{C}$. After removing the solvent, purification was achieved by flash column chromatography (silica, pentane/ethyl acetate 1:1). The title compound $\mathbf{4 b}(4.8 \mathrm{mg}, 19.7 \mu \mathrm{mol}, 79 \%, 80 \% e e)$ was obtained as a colorless solid. 
Enantioselective [2+2] Photocycloaddition (0.15 mmol scale)

2(1H)-Quinolone (22.0 mg, $0.15 \mathrm{mmol}, 1.00$ eq.) and enantiomerically pure $\mathbf{3}$ (6.6 mg, $15.0 \mu \mathrm{mol}, 10 \mathrm{~mol} \%)$ were dissolved in $\alpha, \alpha, \alpha$-trifluorotoluene $(60 \mathrm{~mL}, c=2.5 \mathrm{mmol} / \mathrm{L})$. After degassing the solution, methyl acrylate ( $807 \mu \mathrm{l}, 7.50 \mathrm{mmol}, 50.00$ eq.) was added and the mixture was irradiated at $419 \mathrm{~nm}$ for $8 \mathrm{~h}$ at $-25^{\circ} \mathrm{C}$. After removing the solvent, purification was achieved by flash column chromatography (silica, pentane/ethyl acetate 1:1). The title compound $\mathbf{4 b}(30.3 \mathrm{mg}, 124 \mu \mathrm{mol}, 82 \%, 76 \% e e)$ was obtained as a colorless solid.

Exo isomer $(4 \boldsymbol{b})$ :

M.p.: $111-113{ }^{\circ} \mathrm{C}$.

TLC: $R_{\mathrm{f}}=0.84$ (ethyl acetate, UV).

${ }^{1}$ H-NMR $\left(500 \mathrm{MHz}, \mathrm{CDCl}_{3}, 303 \mathrm{~K}\right): \delta(\mathrm{ppm})=9.37$ (br. s, $\left.1 \mathrm{H}, \mathrm{NH}\right), 7.17$ (virt. t, ${ }^{3} \boldsymbol{J} \approx$ $\left.{ }^{3} J=7.6 \mathrm{~Hz}, 1 \mathrm{H}, \mathrm{H}-6\right), 7.10\left(\mathrm{~d},{ }^{3} J=7.3 \mathrm{~Hz}, 1 \mathrm{H}, \mathrm{H}-8\right), 6.99$ (virt. $\mathrm{t},{ }^{3} J \approx{ }^{3} J=7.4 \mathrm{~Hz}, 1 \mathrm{H}$, $\mathrm{H}-7), 6.84\left(\mathrm{~d},{ }^{3} \mathrm{~J}=7.9 \mathrm{~Hz}, 1 \mathrm{H}, \mathrm{H}-5\right), 4.27-4.09\left(\mathrm{~m}, 2 \mathrm{H}, \mathrm{OCH}_{2} \mathrm{CH}_{3}\right), 4.01$ (virt. t, ${ }^{3} \mathrm{~J} \approx$ $\left.{ }^{3} J=8.8 \mathrm{~Hz}, 1 \mathrm{H}, \mathrm{H}-8 \mathrm{~b}\right), 3.48-3.22(\mathrm{~m}, 2 \mathrm{H}, \mathrm{H}-1, \mathrm{H}-2 \mathrm{a}), 2.87$ (virt. dt, ${ }^{2} J=11.9 \mathrm{~Hz},{ }^{3} J \approx$ $\left.{ }^{3} J=9.8 \mathrm{~Hz}, 1 \mathrm{H}, \mathrm{H}-2\right), 2.64\left(\mathrm{ddd},{ }^{2} J=11.9 \mathrm{~Hz},{ }^{3} J=9.1 \mathrm{~Hz},{ }^{3} J=3.2 \mathrm{~Hz}, 1 \mathrm{H}, \mathrm{H}-2\right), 1.28$ $\left(\mathrm{t}, 3 \mathrm{H},{ }^{3} \mathrm{~J}=7.1 \mathrm{~Hz}, \mathrm{OCH}_{2} \mathrm{CH}_{3}\right)$.

${ }^{13} \mathbf{C}-N M R\left(100 \mathrm{MHz}, \mathrm{CDCl}_{3}, 303 \mathrm{~K}\right): \delta(\mathrm{ppm})=173.5$ (s, COOEt), 173.0 (s, C-3), 136.7 (s, C-4a), 128.5 (d, C-6), 128.0 (d, C-8), 123.9 (d, C-7), 122.6 (s, C-8a), 116.1 (d, C-5), 61.0 (t, $\mathrm{OCH}_{2} \mathrm{CH}_{3}$ ), 45.2 (d, C-2a), 40.1 (d, C-8b), 34.8 (d, C-1), 29.3 (t, C-2), $14.4\left(\mathrm{q}, \mathrm{OCH}_{2} \mathrm{CH}_{3}\right)$.

IR (ATR): $v\left(\mathrm{~cm}^{-1}\right)=3204,3062,2982,1726,1666,1593,1491,1374,1350,1301$, 1239, 1198, 1166, 1063, 1035, 859, 753.

MS (EI, $70 \mathrm{eV}): m / z(\%)=245(7)\left[\mathrm{M}^{+}\right], 199(4)\left[\mathrm{M}^{+}-\mathrm{C}_{2} \mathrm{H}_{5} \mathrm{O}\right], 171$ (11) $\left[\mathrm{M}^{+}-\mathrm{C}_{3} \mathrm{H}_{5} \mathrm{O}_{2}\right]$, 145 (100) $\left[\mathrm{M}^{+}-\mathrm{C}_{5} \mathrm{H}_{8} \mathrm{O}_{2}\right], 117$ (23), 90 (7).

HRMS (EI) $\left(\mathrm{C}_{14} \mathrm{H}_{15} \mathrm{NO}_{3}\right)$ : calc.: 245.1046; found: 245.1052.

HPLC $(A D-H, 250 \times 4.6, n$-hexane/iso-propanol $=90: 10)=4 \mathbf{b}, t_{\mathrm{R}}=12.4 \mathrm{~min} ;$ ent-4b, $13.7 \mathrm{~min})$.

Specific rotation $[\alpha]_{\mathrm{D}}^{20}=-88.6\left(c=0.54, \mathrm{CHCl}_{3}\right)[76 \% e e]$.

Endo isomer (S12):

M.p.: $131-133{ }^{\circ} \mathrm{C}$.

TLC: $R \mathrm{f}=0.54$ (ethyl acetate, UV).

${ }^{1} \mathbf{H}-N M R\left(500 \mathrm{MHz}, \mathrm{CDCl}_{3}, 303 \mathrm{~K}\right): \delta(\mathrm{ppm})=8.21$ (br. s, $\left.1 \mathrm{H}, \mathrm{NH}\right), 7.14$ (virt. td, ${ }^{3} \mathrm{~J} \approx$ $\left.{ }^{3} J=7.7 \mathrm{~Hz},{ }^{4} J=1.5 \mathrm{~Hz}, 1 \mathrm{H}, \mathrm{H}-6\right), 7.00\left(\mathrm{dd},{ }^{3} J=7.6 \mathrm{~Hz},{ }^{4} J=1.5 \mathrm{~Hz}, 1 \mathrm{H}, \mathrm{H}-8\right), 6.93$ (virt. td, $\left.{ }^{3} J \approx{ }^{3} J=7.5 \mathrm{~Hz},{ }^{4} J=1.2 \mathrm{~Hz}, 1 \mathrm{H}, \mathrm{H}-7\right), 6.70\left(\mathrm{dd},{ }^{3} J=8.0 \mathrm{~Hz},{ }^{4} J=1.2 \mathrm{~Hz}, 1 \mathrm{H}\right.$, $\mathrm{H}-5$ ), 4.25 (virt. t, $\left.{ }^{3} J \approx{ }^{3} J=9.8 \mathrm{~Hz}, 1 \mathrm{H}, \mathrm{H}-8 \mathrm{~b}\right), 3.89-3.76\left(\mathrm{~m}, 2 \mathrm{H}, \mathrm{OCH}_{2} \mathrm{CH}_{3}\right), 3.59$ (ddd, ${ }^{3} J=9.9 \mathrm{~Hz},{ }^{3} J=8.5 \mathrm{~Hz},{ }^{3} J=6.8 \mathrm{~Hz}, 1 \mathrm{H}, \mathrm{H}-1$ ), 3.45 (virt. td, ${ }^{3} J \approx{ }^{3} J=9.9 \mathrm{~Hz},{ }^{3} J=$ $6.7 \mathrm{~Hz}, 1 \mathrm{H}, \mathrm{H}-2 \mathrm{a}), 2.88$ (m, 1H, H-2), $2.78-2.67$ (m, 1H, H-2), 0.98 (t, ${ }^{3} \mathrm{~J}=7.1 \mathrm{~Hz}$, $\left.3 \mathrm{H}, \mathrm{OCH}_{2} \mathrm{CH}_{3}\right)$.

${ }^{13}$ C-NMR $\left(100 \mathrm{MHz}, \mathrm{CDCl}_{3}, 303 \mathrm{~K}\right): \delta(\mathrm{ppm})=174.7$ (s, COOEt), 172.0 (s, C-3), 137.2 (s, C-4a), 129.2 (d, C-8), 128.6 (d, C-6), 123.3 (d, C-7), 119.6 (s, C-8a), 115.9 (d, C-5), 60.7 (t, $\mathrm{OCH}_{2} \mathrm{CH}_{3}$ ), 44.2 (d, C-1), 38.8 (d, C-8b), 34.9 (d, C-2a), 27.5 (t, C-2), $14.3\left(\mathrm{q}, \mathrm{OCH}_{2} \mathrm{CH}_{3}\right)$.

IR (ATR): $v\left(\mathrm{~cm}^{-1}\right)=2981,2936,1728,1677,1446,1378,1299,1243,1176,1176$, 1160, 1097, 1023, 853, 757. 
HRMS (EI) $\left(\mathrm{C}_{14} \mathrm{H}_{15} \mathrm{NO}_{3}\right)$ : calc.: 245.1046; found: 245.1049 .

MS (EI, $70 \mathrm{eV}): m / z(\%)=245(5)\left[\mathrm{M}^{+}\right], 199(4)\left[\mathrm{M}^{+}-\mathrm{C}_{2} \mathrm{H}_{5} \mathrm{O}\right], 171(9)\left[\mathrm{M}^{+}-\mathrm{C}_{3} \mathrm{H}_{5} \mathrm{O}_{2}\right]$, 145 (100) $\left[\mathrm{M}^{+}-\mathrm{C}_{5} \mathrm{H}_{8} \mathrm{O}_{2}\right], 117$ (22), 90 (8). 
Benzyl $(1 R, 2 \mathrm{a} R, 8 \mathrm{~b} R)-3-0 x 0-1,2,2 \mathrm{a}, 3,4,8 \mathrm{~b}$-hexahydrocyclobuta[c]quinoline-1-carboxylate (4c) and Benzyl (1S,2aR,8bR)-3-oxo-1,2,2a,3,4,8b-hexahydrocyclobuta[c]quinoline-1-carboxylate (S13)<smiles>O=c1ccc2ccccc2[nH]1</smiles><smiles>C=CC(=O)OCc1ccccc1</smiles>
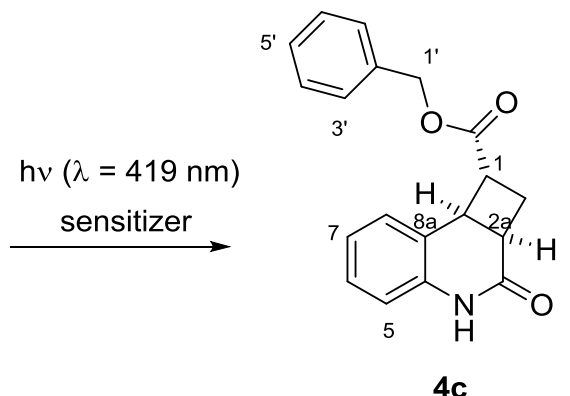<smiles>O=C(OCc1ccccc1)[C@H]1C[C@H]2C(=O)Nc3ccccc3[C@@H]12</smiles>

$\mathrm{C}_{19} \mathrm{H}_{17} \mathrm{NO}_{3}$ MW: $307,35 \mathrm{~g} / \mathrm{mol}$

\section{Racemic [2+2] Photocycloaddition}

2(1H)-Quinolone (20 mg, $0.14 \mathrm{mmol}, 1.00$ eq.) and thioxanthen-9-one (15 mg, $0.07 \mathrm{mmol}, 0.50$ eq.) were dissolved in acetonitrile $(10 \mathrm{~mL}, c=14 \mathrm{mmol} / \mathrm{L})$. After degassing the solution, benzyl acrylate ( $207 \mu \mathrm{l}, 1.4 \mathrm{mmol}, 10.00$ eq.) was added and the mixture was irradiated at $419 \mathrm{~nm}$ for $3 \mathrm{~h}$ at room temperature. After removing the solvent, purification was achieved by flash column chromatography (silica, pentane/ethyl acetate 1:1). The title compounds $\mathbf{4 c}(34.5 \mathrm{mg}, 0.11 \mathrm{mmol}, 79 \%)$ and $\mathbf{S 1 3}$ (7.4 mg, $0.02 \mathrm{mmol}, 17 \%$ ) were obtained as colorless solids.

\section{Enantioselective [2+2] Photocycloaddition}

2(1H)-Quinolone (3.6 mg, $25.0 \mu \mathrm{mol}, 1.00$ eq.) and enantiomerically pure $3(1.1 \mathrm{mg}$, $2.5 \mu \mathrm{mol}, 10 \mathrm{~mol} \%$ ) were dissolved in $\alpha, \alpha, \alpha$-trifluorotoluene $(10 \mathrm{~mL}, c=2.5 \mathrm{mmol} / \mathrm{L})$. After degassing the solution, benyl acrylate (188 $\mu \mathrm{l}, 1.25 \mathrm{mmol}, 50.00$ eq.) was added and the mixture was irradiated at $419 \mathrm{~nm}$ for $6 \mathrm{~h}$ at $-25^{\circ} \mathrm{C}$. After removing the solvent, purification was achieved by flash column chromatography (silica, pentane/ethyl acetate 1:1). The title compound $4 \mathrm{c}(6.4 \mathrm{mg}, 21.0 \mu \mathrm{mol}, 83 \%, 82 \% e e)$ was obtained as a colorless solid.

\section{Enantioselective [2+2] Photocycloaddition (0.15 mmol scale)}

2(1H)-Quinolone (22.0 mg, $0.15 \mathrm{mmol}, 1.00$ eq.) and enantiomerically pure $3(6.6 \mathrm{mg}$, $15.0 \mu \mathrm{mol}, 10 \mathrm{~mol} \%)$ were dissolved in $\alpha, \alpha, \alpha$-trifluorotoluene $(60 \mathrm{~mL}, c=2.5 \mathrm{mmol} / \mathrm{L})$. After degassing the solution, benzyl acrylate $(1.16 \mathrm{ml}, 7.50 \mathrm{mmol}, 50.00$ eq.) was added and the mixture was irradiated at $419 \mathrm{~nm}$ for $8 \mathrm{~h}$ at $-25^{\circ} \mathrm{C}$. After removing the solvent, purification was achieved by flash column chromatography (silica, pentane/ethyl acetate $7: 3 \rightarrow 1: 1)$. The title compound $4 \mathbf{c}(36.3 \mathrm{mg}, 119 \mu \mathrm{mol}, 79 \%, 80 \% e e)$ was obtained as a colorless solid. 
M.p.: $119-121^{\circ} \mathrm{C}$.

TLC: $R_{\mathrm{f}}=0.73$ (ethyl acetate, UV).

${ }^{1} \mathbf{H}-\mathbf{N M R}\left(500 \mathrm{MHz}, \mathrm{CDCl}_{3}, 303 \mathrm{~K}\right): \delta(\mathrm{ppm})=9.36$ (br. s, $\left.1 \mathrm{H}, \mathrm{NH}\right), 7.45-7.30(\mathrm{~m}$, $4 \mathrm{H}, \mathrm{Ar}$ ), $7.23-7.20(\mathrm{~m}, 1 \mathrm{H}, \mathrm{Ar}), 7.17$ (virt. td, ${ }^{3} J \approx{ }^{3} J=7.7 \mathrm{~Hz},{ }^{4} J=1.2 \mathrm{~Hz}, 1 \mathrm{H}, \mathrm{H}-6$ ), $7.01\left(\mathrm{dd},{ }^{3} J=7.1 \mathrm{~Hz},{ }^{4} J=1.2 \mathrm{~Hz}, 1 \mathrm{H}, \mathrm{H}-8\right), 6.95$ (virt. td, ${ }^{3} J \approx{ }^{3} J=7.3 \mathrm{~Hz},{ }^{4} J=1.2 \mathrm{~Hz}$, $1 \mathrm{H}, \mathrm{H}-7), 6.83\left(\mathrm{dd},{ }^{3} J=7.9 \mathrm{~Hz},{ }^{4} J=1.2 \mathrm{~Hz}, 1 \mathrm{H}, \mathrm{H}-5\right), 5.22\left(\mathrm{~d},{ }^{2} J=12.2 \mathrm{~Hz}, 1 \mathrm{H}, \mathrm{H}-1^{\prime}\right)$, $5.13\left(\mathrm{~d},{ }^{2} J=12.2 \mathrm{~Hz}, 1 \mathrm{H}, \mathrm{H}-1\right.$ '), 4.01 (virt. t, ${ }^{3} J \approx{ }^{3} J=8.9 \mathrm{~Hz}, 1 \mathrm{H}, \mathrm{H}-8 \mathrm{~b}$ ), $3.47-3.23$ (m, 2H, H-1, H-2a), 2.90 (virt. dt, ${ }^{2} J=12.1 \mathrm{~Hz},{ }^{3} J \approx{ }^{3} J=9.7 \mathrm{~Hz}, 1 \mathrm{H}, \mathrm{H}-2$ ), 2.65 (ddd, ${ }^{2} J$ $\left.=12.1 \mathrm{~Hz},{ }^{3} J=9.2 \mathrm{~Hz},{ }^{3} J=3.3 \mathrm{~Hz}, 1 \mathrm{H}, \mathrm{H}-2\right)$.

${ }^{13} \mathbf{C}$-NMR $\left(100 \mathrm{MHz}, \mathrm{CDCl}_{3}, 303 \mathrm{~K}\right): \delta(\mathrm{ppm})=173.2(\mathrm{~s}, \mathrm{COOBn}), 172.9$ (s, C-3), 136.8 (s, C-4a), 135.8 (s, C-2'), 128.7 (d, C-4'), 128.5 (d, C-5'), 128.4 (d, C-6), 128.3 (d, C-3'), 128.0 (d, C-8), 123.8 (d, C-7), 122.4 (s, C-8a), 116.0 (d, C-5), 66.7 (t, C-1'), 45.1 (d, C-2a), 40.2 (d, C-8b), 34.9 (d, C-1), 29.2 (t, C-2).

IR (ATR): $v\left(\mathrm{~cm}^{-1}\right)=1728,1665,1593,1493,1456,1382,1350,1250,1236,1195$, 1173, 1151, 1123, 1065, 1025, 907, 870, 854, 831, 758, 701, 679, 620.

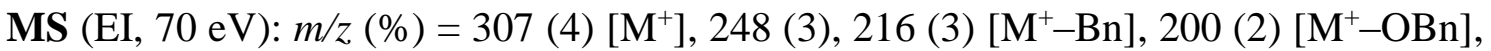
172 (3) $\left[\mathrm{M}^{+}-\mathrm{C}_{8} \mathrm{H}_{7} \mathrm{O}_{2}\right], 159$ (2) $\left[\mathrm{M}^{+}-\mathrm{C}_{9} \mathrm{H}_{9} \mathrm{O}_{2}\right], 145$ (100) $\left[\mathrm{M}^{+}-\mathrm{C}_{10} \mathrm{H}_{11} \mathrm{O}_{2}\right], 117$ (18), 91 (14).

HRMS (EI) $\left(\mathrm{C}_{19} \mathrm{H}_{17} \mathrm{NO}_{3}\right)$ : calc.: 307.1208; found: 307.1216.

HPLC $\left(\right.$ AD-H, 250×4.6, $n$-hexane/iso-propanol = 90:10) $=\mathbf{4 c}, t_{\mathrm{R}}=18.1 \mathrm{~min} ;$ ent-4c, $24.2 \mathrm{~min})$.

Specific rotation $[\alpha]_{\mathrm{D}}^{20}=-86.8\left(c=0.67, \mathrm{CHCl}_{3}\right)[80 \% e e]$.

Endo isomer (S13):

M.p.: $121-123{ }^{\circ} \mathrm{C}$.

TLC: $R \mathrm{f}=0.64$ (ethyl acetate, UV).

${ }^{1} \mathbf{H}$-NMR $\left(500 \mathrm{MHz}, \mathrm{CDCl}_{3}, 303 \mathrm{~K}\right): \delta(\mathrm{ppm})=8.03$ (br. s, $\left.1 \mathrm{H}, \mathrm{NH}\right), 7.39-7.26(\mathrm{~m}$, $3 \mathrm{H}, \mathrm{Ar}), 7.26-7.06$ (m, 3H, Ar, H-6), $6.94\left(\mathrm{dd},{ }^{3} J=7.7 \mathrm{~Hz},{ }^{4} J=1.6 \mathrm{~Hz}, 1 \mathrm{H}, \mathrm{H}-8\right), 6.86$ (virt. td, $\left.{ }^{3} J \approx{ }^{3} J=7.4 \mathrm{~Hz},{ }^{4} J=1.1 \mathrm{~Hz}, 1 \mathrm{H}, \mathrm{H}-7\right), 6.65\left(\mathrm{dd},{ }^{3} J=8.0 \mathrm{~Hz},{ }^{4} J=1.1 \mathrm{~Hz}, 1 \mathrm{H}\right.$, H-5), $4.85\left(\mathrm{~d},{ }^{2} J=12.1 \mathrm{~Hz}, 1 \mathrm{H}, \mathrm{H}-1^{\prime}\right), 4.71\left(\mathrm{~d},{ }^{2} J=12.1 \mathrm{~Hz}, 1 \mathrm{H}, \mathrm{H}-1^{\prime}\right), 4.25$ (virt. t, ${ }^{3} J \approx$ $\left.{ }^{3} J=9.9 \mathrm{~Hz}, 1 \mathrm{H}, \mathrm{H}-8 \mathrm{~b}\right), 3.66\left(\mathrm{ddd},{ }^{3} J=9.8 \mathrm{~Hz},{ }^{3} J=8.5 \mathrm{~Hz},{ }^{3} J=6.9 \mathrm{~Hz}, 1 \mathrm{H}, \mathrm{H}-1\right), 3.45$ (virt. td, ${ }^{3} J \approx{ }^{3} J=9.9 \mathrm{~Hz},{ }^{3} J=6.8 \mathrm{~Hz}, 1 \mathrm{H}, \mathrm{H}-2 \mathrm{a}$ ), 2.91 (virt. dt, ${ }^{2} J=12.4 \mathrm{~Hz},{ }^{3} J \approx{ }^{3} J=$ $6.9 \mathrm{~Hz}, 1 \mathrm{H}, \mathrm{H}-2), 2.82-2.64(\mathrm{~m}, 1 \mathrm{H}, \mathrm{H}-2)$.

${ }^{13} \mathbf{C}-\mathbf{N M R}\left(100 \mathrm{MHz}, \mathrm{CDCl}_{3}, 303 \mathrm{~K}\right): \delta(\mathrm{ppm})=171.8$ (s, COOBn), 170.9 (s, C-3), 137.3 (s, C-4a), 135.5 (s, C-2'), 129.1 (d, C-5'), 128.7 (d, C-6), 128.6 (d, C-4'), 128.6 (d, C-3'), 128.4 (d, C-8), 123.3 (d, C-7), 119.4 (s, C-8a), 115.9 (d, C-5), 66.7 (t, C-1'), 44.2 (d, C-1), 39.0 (d, C-8b), 34.9 (d, C-2a), 27.6 (t, C-2).

IR (ATR): $v\left(\mathrm{~cm}^{-1}\right)=1728,1713,1685,1594,1495,1455,1383,1348,1322,1308$, 1251, 1185, 1173, 1152, 1124, 1094, 1065, 1037, 1011, 946, 909, 871, 853, 810, 752, $685,666,648,620$.

MS (EI, $70 \mathrm{eV}): m / z(\%)=307(3)\left[\mathrm{M}^{+}\right], 248(2), 216(2)\left[\mathrm{M}^{+}-\mathrm{Bn}\right], 200(1)\left[\mathrm{M}^{+}-\mathrm{OBn}\right]$, 172 (2) $\left[\mathrm{M}^{+}-\mathrm{C}_{8} \mathrm{H}_{7} \mathrm{O}_{2}\right], 159$ (1) $\left[\mathrm{M}^{+}-\mathrm{C}_{9} \mathrm{H}_{9} \mathrm{O}_{2}\right], 145$ (100) $\left[\mathrm{M}^{+}-\mathrm{C}_{10} \mathrm{H}_{11} \mathrm{O}_{2}\right], 117$ (18), 91 (16).

HRMS (EI) $\left(\mathrm{C}_{19} \mathrm{H}_{17} \mathrm{NO}_{3}\right)$ : calc.: 307.1208; found: 307.1203. 
Benzyl (1R,2a $R, 8 \mathrm{~b} S)$-1-methyl-3-oxo-1,2,2a,3,4,8b-hexahydrocyclobuta[c]quinoline-1-carboxylate (4d) and Benzyl (1S,2aR,8bS)-1-methyl-3-oxo-1,2,2a,3,4,8bhexahydrocyclobuta[c]quinoline-1-carboxylate (S14)<smiles>O=c1ccc2ccccc2[nH]1</smiles><smiles>C=C(C)C(=O)OCc1ccccc1</smiles>

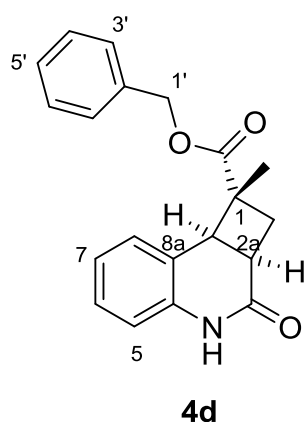

4d<smiles>CCCCCCC(=O)OCc1ccccc1</smiles>

$\mathrm{C}_{20} \mathrm{H}_{19} \mathrm{NO}_{3}$ $\mathrm{MW}: 321,38 \mathrm{~g} / \mathrm{mol}$

\section{Racemic [2+2] Photocycloaddition}

2(1H)-Quinolone (20 mg, $0.14 \mathrm{mmol}, 1.00$ eq.) and thioxanthen-9-one (15 mg, $0.07 \mathrm{mmol}, 0.50$ eq.) were dissolved in acetonitrile $(10 \mathrm{~mL}, c=14 \mathrm{mmol} / \mathrm{L})$. After degassing the solution, benzyl methacrylate $(234 \mu \mathrm{l}, 1.4 \mathrm{mmol}, 10.00$ eq.) was added and the mixture was irradiated at $419 \mathrm{~nm}$ for $6 \mathrm{~h}$ at room temperature. After removing the solvent, purification was achieved by flash column chromatography (silica, pentane/ethyl acetate 1:1). The title compounds $4 \mathbf{d}(28.2 \mathrm{mg}, 0.09 \mathrm{mmol}, 64 \%)$ and $\mathbf{S 1 4}$ $(6.8 \mathrm{mg}, 0.02 \mathrm{mmol}, 15 \%)$ were obtained as colorless solids.

\section{Enantioselective [2+2] Photocycloaddition}

2(1H)-Quinolone (3.6 mg, $25.0 \mu \mathrm{mol}, 1.00$ eq.) and enantiomerically pure 3 (1.1 mg, $2.5 \mu \mathrm{mol}, 10 \mathrm{~mol} \%$ ) were dissolved in $\alpha, \alpha, \alpha$-trifluorotoluene $(10 \mathrm{~mL}, c=2.5 \mathrm{mmol} / \mathrm{L})$. After degassing the solution, benzyl methacrylate $(212 \mu \mathrm{l}, 1.25 \mathrm{mmol}, 50.00$ eq.) was added and the mixture was irradiated at $419 \mathrm{~nm}$ for $6 \mathrm{~h}$ at $-25{ }^{\circ} \mathrm{C}$. After removing the solvent, purification was achieved by flash column chromatography (silica, pentane/ethyl acetate 1:1). The title compounds $4 \mathbf{d}$ (5.3 mg, $16.0 \mu \mathrm{mol}, 66 \%, 90 \%$ ee ) and S14 (1.4 mg, $4.0 \mu \mathrm{mol}, 17 \%, 91 \% e e$ ) were obtained as colorless solids.

\section{Enantioselective [2+2] Photocycloaddition (0.15 mmol scale)}

2(1H)-Quinolone (22.0 mg, $0.15 \mathrm{mmol}, 1.00$ eq.) and enantiomerically pure 3 (6.6 mg, $15.0 \mu \mathrm{mol}, 10 \mathrm{~mol} \%)$ were dissolved in $\alpha, \alpha, \alpha$-trifluorotoluene $(60 \mathrm{~mL}, c=2.5 \mathrm{mmol} / \mathrm{L})$. After degassing the solution, benzyl methacrylate $(1.28 \mathrm{ml}, 7.50 \mathrm{mmol}, 50.00$ eq.) was added and the mixture was irradiated at $419 \mathrm{~nm}$ for $8 \mathrm{~h}$ at $-25{ }^{\circ} \mathrm{C}$. After removing the solvent, purification was achieved by flash column chromatography (silica, pentane/ethyl acetate 7:3 $\rightarrow$ 1:1). The title compounds 4 d (37.2 mg, $116 \mu \mathrm{mol}, 76 \%$, $88 \% e e$ ) and S14 (7.1 mg, $22.0 \mu \mathrm{mol}, 15 \%, 85 \% e e$ ) were obtained as colorless solids. 
M.p.: $157-159^{\circ} \mathrm{C}$.

TLC: $R_{\mathrm{f}}=0.78$ (ethyl acetate, UV).

${ }^{1} \mathbf{H}-\mathbf{N M R}\left(500 \mathrm{MHz}, \mathrm{CDCl}_{3}, 303 \mathrm{~K}\right): \delta(\mathrm{ppm})=9.11$ (br. s, $\left.1 \mathrm{H}, \mathrm{NH}\right), 7.44-7.31(\mathrm{~m}$, $5 \mathrm{H}, \mathrm{Ar}$ ), 7.16 (virt. t, $\left.{ }^{3} J \approx{ }^{3} J=7.6 \mathrm{~Hz}, 1 \mathrm{H}, \mathrm{H}-6\right), 6.99\left(\mathrm{~d},{ }^{3} J=6.8 \mathrm{~Hz}, 1 \mathrm{H}, \mathrm{H}-8\right), 6.95$ (virt. $\left.\mathrm{t},{ }^{3} J \approx{ }^{3} J=7.1 \mathrm{~Hz}, 1 \mathrm{H}, \mathrm{H}-7\right), 6.80\left(\mathrm{~d},{ }^{3} J=7.9 \mathrm{~Hz}, 1 \mathrm{H}, \mathrm{H}-5\right), 5.23\left(\mathrm{~d},{ }^{2} J=12.4 \mathrm{~Hz}\right.$, $\left.1 \mathrm{H}, \mathrm{H}-1^{\prime}\right), 5.18\left(\mathrm{~d},{ }^{2} J=12.4 \mathrm{~Hz}, 1 \mathrm{H}, \mathrm{H}-1^{\prime}\right), 4.20$ (d, ${ }^{3} J=10.4 \mathrm{~Hz}, 1 \mathrm{H}, \mathrm{H}-8 \mathrm{~b}$ ), 3.33 (virt. $\left.\mathrm{td},{ }^{3} J \approx{ }^{3} J=10.6 \mathrm{~Hz},{ }^{3} J=3.9 \mathrm{~Hz}, 1 \mathrm{H}, \mathrm{H}-2 \mathrm{a}\right), 3.19\left(\mathrm{dd},{ }^{2} J=12.5 \mathrm{~Hz},{ }^{3} J=10.8 \mathrm{~Hz}, 1 \mathrm{H}\right.$, $\mathrm{H}-2), 2.30\left(\mathrm{dd},{ }^{2} J=12.5 \mathrm{~Hz},{ }^{3} J=3.9 \mathrm{~Hz}, 1 \mathrm{H}, \mathrm{H}-2\right), 1.16$ (s, 3H, Me).

${ }^{13}$ C-NMR $\left(100 \mathrm{MHz}, \mathrm{CDCl}_{3}, 303 \mathrm{~K}\right): \delta(\mathrm{ppm})=176.4(\mathrm{~s}, \mathrm{COOBn}), 173.2$ (s, C-3), 138.0 (s, C-4a), 136.2 (s, C-2'), 129.3 (d, C-5'), 128.9 (d, C-4'), 128.6 (d, C-6), 128.6 (d, C-3'), 128.3 (d, C-8), 123.7 (d, C-7), 119.5 (s, C-8a), 115.9 (d, C-5), 66.9 (t, C-1'), 47.9 (s, C-1), 42.5 (d, C-8b), 36.4 (t, C-2), 32.5 (d, C-2a), 20.3 (q, Me).

IR (ATR): $v\left(\mathrm{~cm}^{-1}\right)=1728,1667,1594,1456,1382,1350,1323,1308,1250,1236$, 1194, 1173, 1151, 1124, 1093, 1065, 1037, 1026, 908, 870, 830, 755, 696, 679, 667, 620.

MS (EI, $70 \mathrm{eV}): m / z(\%)=321(5)\left[\mathrm{M}^{+}\right], 176(8), 158(8), 145(100)\left[\mathrm{M}^{+}-\mathrm{C}_{11} \mathrm{H}_{12} \mathrm{O}_{2}\right]$, 131 (20), 117 (33), 91 (49), 69 (17).

HRMS (EI) $\left(\mathrm{C}_{20} \mathrm{H}_{19} \mathrm{NO}_{3}\right)$ : calc.: 321.1359 ; found: 321.1361 .

HPLC $($ AD-H, 250×4.6, $n$-hexane/iso-propanol $=90: 10)=4 \mathbf{d}, t_{\mathrm{R}}=14.4 \mathrm{~min} ;$ ent-4d, $19.6 \mathrm{~min})$.

Specific rotation $[\alpha]_{\mathrm{D}}{ }^{20}=-74.9\left(c=0.67, \mathrm{CHCl}_{3}\right)[88 \% e e]$.

Endo isomer (S14):

M.p.: $162-164{ }^{\circ} \mathrm{C}$.

TLC: $R_{\mathrm{f}}=0.72$ (ethyl acetate, UV).

${ }^{1}$ H-NMR $\left(500 \mathrm{MHz}, \mathrm{CDCl}_{3}, 303 \mathrm{~K}\right): \delta(\mathrm{ppm})=7.89$ (br. s, $\left.1 \mathrm{H}, \mathrm{NH}\right), 7.33-7.26(\mathrm{~m}$, $3 \mathrm{H}, \mathrm{Ar}$ ), $7.14-7.05(\mathrm{~m}, 3 \mathrm{H}, \mathrm{Ar}, \mathrm{H}-6), 6.95\left(\mathrm{~d},{ }^{3} \mathrm{~J}=7.4 \mathrm{~Hz}, 1 \mathrm{H}, \mathrm{H}-8\right), 6.87$ (virt. $\mathrm{t},{ }^{3} J \approx$ $\left.{ }^{3} J=7.4 \mathrm{~Hz}, 1 \mathrm{H}, \mathrm{H}-7\right), 6.55\left(\mathrm{~d},{ }^{3} J=7.9 \mathrm{~Hz}, 1 \mathrm{H}, \mathrm{H}-5\right), 4.69\left(\mathrm{~d},{ }^{2} J=12.3 \mathrm{~Hz}, 1 \mathrm{H}, \mathrm{H}-1^{\prime}\right)$, $4.65\left(\mathrm{~d},{ }^{2} J=12.3 \mathrm{~Hz}, 1 \mathrm{H}, \mathrm{H}-1\right.$ '), $3.76\left(\mathrm{~d},{ }^{3} J=10.1 \mathrm{~Hz}, 1 \mathrm{H}, \mathrm{H}-8 \mathrm{~b}\right), 3.44$ (virt. $\mathrm{td},{ }^{3} J \approx{ }^{3} J=$ $\left.10.2 \mathrm{~Hz},{ }^{3} J=5.8 \mathrm{~Hz}, 1 \mathrm{H}, \mathrm{H}-2 \mathrm{a}\right), 3.17\left(\mathrm{dd},{ }^{2} J=12.5 \mathrm{~Hz},{ }^{3} J=5.8 \mathrm{~Hz}, 1 \mathrm{H}, \mathrm{H}-2\right), 2.44$ (virt. $\left.\mathrm{t},{ }^{2} J \approx^{3} J=11.4 \mathrm{~Hz}, 1 \mathrm{H}, \mathrm{H}-2\right), 1.62$ (s, 3H, Me).

${ }^{13} \mathrm{C}$-NMR $\left(100 \mathrm{MHz}, \mathrm{CDCl}_{3}, 303 \mathrm{~K}\right): \delta(\mathrm{ppm})=173.8$ (s, COOBn), 171.5 (s, C-3), 137.2 (s, C-4a), 135.7 (s, C-2'), 128.9 (d, C-5'), 128.7 (d, C-6), 128.6 (d, C-4'), 128.5 (d, C-3'), 128.4 (d, C-8), 123.3 (d, C-7), 120.0 (s, C-8a), 115.8 (d, C-5), 66.8 (t, C-1'), 50.9 (s, C-1), 47.3 (d, C-8b), 35.2 (t, C-2), 32.5 (d, C-2a), 25.5 (q, Me).

IR (ATR): $v\left(\mathrm{~cm}^{-1}\right)=1729,1715,1667,1594,1494,1456,1382,1350,1323,1308$, 1250, 1236, 1187, 173, 1153, 1124, 1065, 911, 755, 696, 678, 667, 621, 601.

MS (EI, $70 \mathrm{eV}): m / z(\%)=321(5)\left[\mathrm{M}^{+}\right], 176(7), 158(8), 145(100)\left[\mathrm{M}^{+}-\mathrm{C}_{11} \mathrm{H}_{12} \mathrm{O}_{2}\right]$, 131 (22), 117 (34), 91 (46), 69 (12).

HRMS (EI) $\left(\mathrm{C}_{20} \mathrm{H}_{19} \mathrm{NO}_{3}\right)$ : calc.: 321.1359 ; found: 321.1365 .

HPLC (AD-H, 250×4.6, $n$-hexane/iso-propanol = 80:20) $=\mathbf{S 1 3}, t_{\mathrm{R}}=13.0 \mathrm{~min}$; ent-S13, $22.8 \mathrm{~min})$.

Specific rotation $[\alpha]_{\mathrm{D}}^{20}=-43.0\left(c=1.53, \mathrm{CHCl}_{3}\right)[85 \% e e]$. 

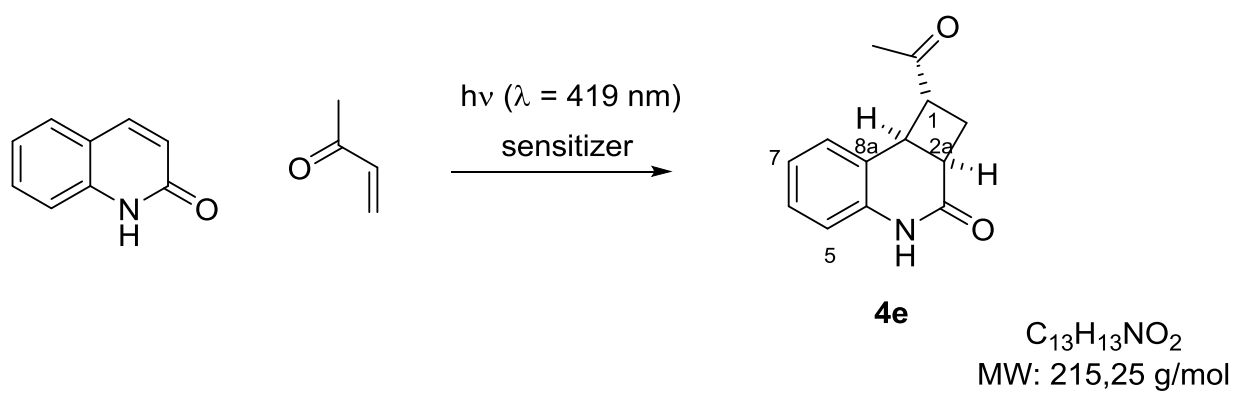

\section{Racemic [2+2] Photocycloaddition}

2(1H)-Quinolone (20 mg, $0.14 \mathrm{mmol}, 1.00$ eq.) and thioxanthen-9-one (15 mg, $0.07 \mathrm{mmol}, 0.50$ eq.) were dissolved in acetonitrile $(10 \mathrm{~mL}, c=14 \mathrm{mmol} / \mathrm{L})$. After degassing the solution, 3-buten-2-one (115 $\mu \mathrm{l}, 1.4 \mathrm{mmol}, 10.00$ eq. $)$ was added and the mixture was irradiated at $419 \mathrm{~nm}$ for $3 \mathrm{~h}$ at room temperature. After removing the solvent, purification was achieved by flash column chromatography (silica, pentane/ethyl acetate 1:1). The title compound $4 \mathrm{e}(21.0 \mathrm{mg}, 0.10 \mathrm{mmol}, 70 \%)$ was obtained as a colorless solid.

\section{Enantioselective [2+2] Photocycloaddition}

2(1H)-Quinolone (3.6 mg, $25.0 \mu \mathrm{mol}, 1.00 \mathrm{eq}$.) and enantiomerically pure $3(1.1 \mathrm{mg}$, $2.5 \mu \mathrm{mol}, 10 \mathrm{~mol} \%$ ) were dissolved in $\alpha, \alpha, \alpha$-trifluorotoluene $(10 \mathrm{~mL}, c=2.5 \mathrm{mmol} / \mathrm{L})$. After degassing the solution, 3-buten-2-one (104 $\mu \mathrm{l}, 1.25 \mathrm{mmol}, 50.00$ eq.) was added and the mixture was irradiated at $419 \mathrm{~nm}$ for $7 \mathrm{~h}$ at $-25^{\circ} \mathrm{C}$. After removing the solvent, purification was achieved by flash column chromatography (silica, pentane/ethyl acetate 1:1). The title compound $4 \mathrm{e}(4.3 \mathrm{mg}, 20.0 \mu \mathrm{mol}, 80 \%, 91 \% e e)$ was obtained as a colorless solid.

M.p.: $157-159^{\circ} \mathrm{C}$.

TLC: $R_{\mathrm{f}}=0.62$ (ethyl acetate, UV).

${ }^{1}$ H-NMR $\left(500 \mathrm{MHz}, \mathrm{CDCl}_{3}, 303 \mathrm{~K}\right): \delta(\mathrm{ppm})=9.52$ (br. s, $\left.1 \mathrm{H}, \mathrm{NH}\right), 7.18$ (virt. td, ${ }^{3} \boldsymbol{J} \approx$ $\left.{ }^{3} J=7.6 \mathrm{~Hz},{ }^{4} J=1.6 \mathrm{~Hz}, 1 \mathrm{H}, \mathrm{H}-6\right), 7.09\left(\mathrm{dd},{ }^{3} J=7.5 \mathrm{~Hz},{ }^{4} J=1.6 \mathrm{~Hz}, 1 \mathrm{H}, \mathrm{H}-8\right), 6.99$ (virt. td, $\left.{ }^{3} J \approx{ }^{3} J=7.4 \mathrm{~Hz},{ }^{4} J=1.2 \mathrm{~Hz}, 1 \mathrm{H}, \mathrm{H}-7\right), 6.87\left(\mathrm{dd},{ }^{3} J=7.9 \mathrm{~Hz},{ }^{4} J=1.2 \mathrm{~Hz}, 1 \mathrm{H}\right.$, H-5), 3.97 (virt. t, ${ }^{3} J \approx{ }^{3} J=8.8 \mathrm{~Hz}, 1 \mathrm{H}, \mathrm{H}-8 \mathrm{~b}$ ), 3.47 (ddd, ${ }^{3} \mathrm{~J}=11.6 \mathrm{~Hz},{ }^{3} \mathrm{~J}=9.2 \mathrm{~Hz},{ }^{3} \mathrm{~J}=$ $8.5 \mathrm{~Hz}, 1 \mathrm{H}, \mathrm{H}-1), 3.24$ (ddd, ${ }^{3} J=9.8 \mathrm{~Hz},{ }^{3} J=9.1 \mathrm{~Hz},{ }^{3} J=3.1 \mathrm{~Hz}, 1 \mathrm{H}, \mathrm{H}-2 \mathrm{a}$ ), 2.80 (virt. $\left.\mathrm{td},{ }^{2} J \approx{ }^{3} J=11.8 \mathrm{~Hz},{ }^{3} J=9.8 \mathrm{~Hz}, 1 \mathrm{H}, \mathrm{H}-2\right), 2.60\left(\mathrm{ddd},{ }^{2} J=12.0 \mathrm{~Hz},{ }^{3} J=9.2 \mathrm{~Hz},{ }^{3} J=\right.$ $3.1 \mathrm{~Hz}, 1 \mathrm{H}, \mathrm{H}-2), 2.10$ (s, 3H, Me).

${ }^{13}$ C-NMR $\left(100 \mathrm{MHz}, \mathrm{CDCl}_{3}, 303 \mathrm{~K}\right): \delta(\mathrm{ppm})=207.1(\mathrm{~s}, \mathrm{COMe}), 173.2(\mathrm{~s}, \mathrm{C}-3), 136.9$ (s, C-4a), 128.5 (d, C-6), 127.9 (d, C-8), 123.9 (d, C-7), 122.8 (s, C-8a), 116.1 (d, C-5), 52.8 (d, C-1), 39.1 (d, C-8b), 34.3 (d, C-2a), 28.8 (t, C-2), 28.2 (q, Me).

IR (ATR): $v\left(\mathrm{~cm}^{-1}\right)=1699,1662,1592,1492,1440,1388,1363,1317,1302,1250$, 1237, 1189, 1175, 948, 932, 869, 833, 804, 752, 704, 607, 637, 620, 601.

MS (EI, $70 \mathrm{eV}): m / z(\%)=215(7)\left[\mathrm{M}^{+}\right], 200(1)\left[\mathrm{M}^{+}-\mathrm{Me}\right], 172(55)\left[\mathrm{M}^{+}-\mathrm{C}_{2} \mathrm{H}_{3} \mathrm{O}\right], 154$ (4), 145 (100) $\left[\mathrm{M}^{+}-\mathrm{C}_{4} \mathrm{H}_{6} \mathrm{O}\right], 117$ (37), 90 (12). 
HRMS (EI) $\left(\mathrm{C}_{13} \mathrm{H}_{13} \mathrm{NO}_{2}\right)$ : calc.: 215.0941; found: 215.0935 .

HPLC (OJ-H, 250×4.6, $n$-hexane/iso-propanol $=80: 20)=$ ent-4e, $t_{\mathrm{R}}=17.5 \mathrm{~min} ; \mathbf{4 e}$, $20.9 \mathrm{~min})$. 


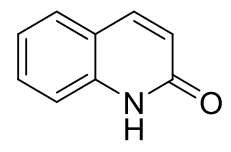<smiles>C=CC(=O)CC</smiles>
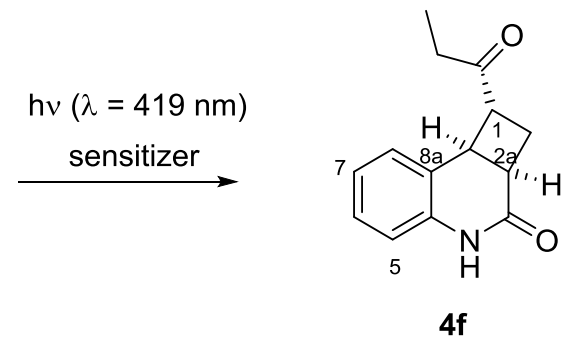

$\mathrm{C}_{14} \mathrm{H}_{15} \mathrm{NO}_{2}$

MW: $229,28 \mathrm{~g} / \mathrm{mol}$

\section{Racemic [2+2] Photocycloaddition}

2(1H)-Quinolone (20 mg, $0.14 \mathrm{mmol}, 1.00$ eq.) and thioxanthen-9-one (15 mg, $0.07 \mathrm{mmol}, 0.50$ eq.) were dissolved in acetonitrile $(10 \mathrm{~mL}, c=14 \mathrm{mmol} / \mathrm{L})$. After degassing the solution, 1-penten-3-one (136 $\mu \mathrm{l}, 1.4 \mathrm{mmol}, 10.00$ eq.) was added and the mixture was irradiated at $419 \mathrm{~nm}$ for $3 \mathrm{~h}$ at room temperature. After removing the solvent, purification was achieved by flash column chromatography (silica, pentane/ethyl acetate 1:1). The title compound $\mathbf{4 f}(22.9 \mathrm{mg}, 0.10 \mathrm{mmol}, 72 \%)$ was obtained as a colorless solid.

\section{Enantioselective [2+2] Photocycloaddition}

2(1H)-Quinolone (3.6 mg, $25.0 \mu \mathrm{mol}, 1.00$ eq.) and enantiomerically pure $3(1.1 \mathrm{mg}$, $2.5 \mu \mathrm{mol}, 10 \mathrm{~mol} \%)$ were dissolved in $\alpha, \alpha, \alpha$-trifluorotoluene $(10 \mathrm{~mL}, c=2.5 \mathrm{mmol} / \mathrm{L})$. After degassing the solution, 1-penten-3-one (124 $\mu$ l, $1.25 \mathrm{mmol}, 50.00$ eq.) was added and the mixture was irradiated at $419 \mathrm{~nm}$ for $6 \mathrm{~h}$ at $-25^{\circ} \mathrm{C}$. After removing the solvent, purification was achieved by flash column chromatography (silica, pentane/ethyl acetate 1:1). The title compound $\mathbf{4 f}(4.0 \mathrm{mg}, 18.0 \mu \mathrm{mol}, 70 \%, 92 \% e e)$ was obtained as a colorless solid.

\section{Enantioselective [2+2] Photocycloaddition (0.15 mmol scale)}

2(1H)-Quinolone (22.0 mg, $0.15 \mathrm{mmol}, 1.00$ eq.) and enantiomerically pure $3(6.6 \mathrm{mg}$, $15.0 \mu \mathrm{mol}, 10 \mathrm{~mol} \%)$ were dissolved in $\alpha, \alpha, \alpha$-trifluorotoluene $(60 \mathrm{~mL}, c=2.5 \mathrm{mmol} / \mathrm{L})$. After degassing the solution, 1-penten-3-one $(750 \mu 1,7.50 \mathrm{mmol}, 50.00 \mathrm{eq}$.) was added and the mixture was irradiated at $419 \mathrm{~nm}$ for $12 \mathrm{~h}$ at $-25{ }^{\circ} \mathrm{C}$. After removing the solvent, purification was achieved by flash column chromatography (silica, pentane/ethyl acetate $2: 1 \rightarrow 1: 1)$. The title compound 4 f $(30.9 \mathrm{mg}, 135 \mu \mathrm{mol}, 89 \%$, $91 \%$ ee) was obtained as a colorless solid.

M.p.: $154-156^{\circ} \mathrm{C}$.

TLC: $R_{\mathrm{f}}=0.76$ (ethyl acetate, UV).

${ }^{\mathbf{1}} \mathbf{H}-\mathbf{N M R}\left(500 \mathrm{MHz}, \mathrm{CDCl}_{3}, 303 \mathrm{~K}\right): \delta(\mathrm{ppm})=9.38$ (br. s, $\left.1 \mathrm{H}, \mathrm{NH}\right), 7.17$ (virt. td, ${ }^{3} \boldsymbol{J} \approx$ $\left.{ }^{3} J=7.7 \mathrm{~Hz},{ }^{4} J=1.6 \mathrm{~Hz}, 1 \mathrm{H}, \mathrm{H}-6\right), 7.07\left(\mathrm{dd},{ }^{3} J=7.5 \mathrm{~Hz},{ }^{4} J=1.6 \mathrm{~Hz}, 1 \mathrm{H}, \mathrm{H}-8\right), 6.97$ (virt. td, $\left.{ }^{3} J \approx{ }^{3} J=7.5 \mathrm{~Hz},{ }^{4} J=1.2 \mathrm{~Hz}, 1 \mathrm{H}, \mathrm{H}-7\right), 6.84\left(\mathrm{dd},{ }^{3} J=7.9 \mathrm{~Hz},{ }^{4} J=1.2 \mathrm{~Hz}, 1 \mathrm{H}\right.$, $\mathrm{H}-5$ ), 3.98 (virt. t, ${ }^{3} J \approx{ }^{3} J=8.8 \mathrm{~Hz}, 1 \mathrm{H}, \mathrm{H}-8 \mathrm{~b}$ ), 3.47 (virt. dt, ${ }^{3} J=9.8 \mathrm{~Hz},{ }^{3} J \approx{ }^{3} J=8.0$ 
$\mathrm{Hz}, 1 \mathrm{H}, \mathrm{H}-1$ ), 3.24 (ddd, ${ }^{3} \mathrm{~J}=9.8 \mathrm{~Hz},{ }^{3} \mathrm{~J}=9.1 \mathrm{~Hz},{ }^{3} \mathrm{~J}=3.1 \mathrm{~Hz}, 1 \mathrm{H}, \mathrm{H}-2 \mathrm{a}$ ), 2.78 (virt. td, $\left.{ }^{2} J \approx{ }^{3} J=11.9 \mathrm{~Hz},{ }^{3} J=9.8 \mathrm{~Hz}, 1 \mathrm{H}, \mathrm{H}-2\right), 2.59\left(\mathrm{ddd},{ }^{2} J=12.0 \mathrm{~Hz},{ }^{3} J=9.1 \mathrm{~Hz},{ }^{3} J=3.0\right.$ $\mathrm{Hz}, 1 \mathrm{H}, \mathrm{H}-2), 2.45-2.22\left(\mathrm{~m}, 2 \mathrm{H}, \mathrm{CH}_{2} \mathrm{CH}_{3}\right), 1.05\left(\mathrm{t},{ }^{3} \mathrm{~J}=7.3 \mathrm{~Hz}, 3 \mathrm{H}, \mathrm{CH}_{2} \mathrm{CH}_{3}\right)$.

${ }^{13} \mathrm{C}$-NMR $\left(100 \mathrm{MHz}, \mathrm{CDCl}_{3}, 303 \mathrm{~K}\right): \delta(\mathrm{ppm})=210.0(\mathrm{~s}, \mathrm{COMe}), 173.2$ (s, C-3), 137.1 (s, C-4a), 128.5 (d, C-6), 128.0 (d, C-8), 123.9 (d, C-7), 123.0 (s, C-8a), 116.1 (d, C-5), 51.9 (d, C-1), 39.3 (d, C-8b), 34.6 (d, C-2a), 34.4 (t, $\left.\mathrm{CH}_{2} \mathrm{CH}_{3}\right), 29.2$ (t, C-2), 7.7 (q, $\left.\mathrm{CH}_{2} \mathrm{CH}_{3}\right)$.

IR (ATR): $v\left(\mathrm{~cm}^{-1}\right)=1699,1662,1592,1492,1440,1388,1363,1317,1302,1250$, 1237, 1189, 1175, 948, 932, 869, 833, 804, 752, 704, 607, 637, 620, 601.

MS (EI, $70 \mathrm{eV}): m / z(\%)=229(8)\left[\mathrm{M}^{+}\right], 200(1)\left[\mathrm{M}^{+}-\mathrm{C}_{2} \mathrm{H}_{5}\right], 172(26)\left[\mathrm{M}^{+}-\mathrm{C}_{3} \mathrm{H}_{5} \mathrm{O}\right]$, 154 (4), 145 (100) [ $\left.\mathrm{M}^{+}-\mathrm{C}_{5} \mathrm{H}_{8} \mathrm{O}\right], 128$ (4), 117 (18), 90 (7), 77 (3).

HRMS (EI) $\left(\mathrm{C}_{14} \mathrm{H}_{15} \mathrm{NO}_{2}\right)$ : calc.: 229.1097; found: 229.1096 .

HPLC $(\mathrm{AD}-\mathrm{H}, 250 \times 4.6, n$-hexane/iso-propanol $=90: 10)=\mathbf{4 f}, t_{\mathrm{R}}=15.4 \mathrm{~min}$; ent-4f, $16.9 \mathrm{~min})$.

Specific rotation $[\alpha]_{\mathrm{D}}^{20}=-93.9(\mathrm{c}=0.50, \mathrm{MeOH})[88 \% e e]$. 
$(1 S, 2 \mathrm{a} S, 8 \mathrm{~b} S)$-1-Acetyl-2,2-dimethyl-2,2a,4,8b-tetrahydrocyclobuta[c]quinolin3(1H)-one $(4 \mathrm{~g}),(2 R, 2 \mathrm{a} S, 8 \mathrm{~b} R)$-2-acetyl-1,1-dimethyl-2,2a,4,8b-tetrahydrocyclobuta[c]quinolin-3(1H)-one (S15) and (2S,2aS,8bR)-2-acetyl-1,1-dimethyl-2,2a,4,8btetrahydrocyclobuta[c]quinolin-3(1H)-one (S16)<smiles>O=c1ccc2ccccc2[nH]1</smiles>
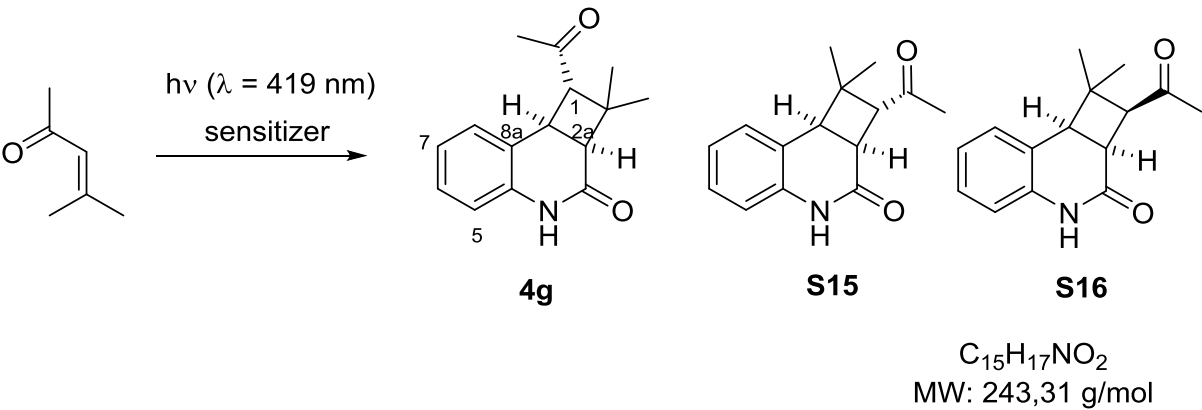

Racemic [2+2] Photocycloaddition

2(1H)-Quinolone (20 mg, $0.14 \mathrm{mmol}, 1.00$ eq.) and thioxanthen-9-one (15 mg, $0.07 \mathrm{mmol}, 0.50$ eq.) were dissolved in acetonitrile $(10 \mathrm{~mL}, c=14 \mathrm{mmol} / \mathrm{L})$. After degassing the solution, mesityl oxide ( $158 \mu \mathrm{l}, 1.4 \mathrm{mmol}, 10.00$ eq.) was added and the mixture was irradiated at $419 \mathrm{~nm}$ for $22 \mathrm{~h}$ at room temperature (92\% conversion). After removing the solvent, purification was achieved by flash column chromatography (silica, pentane/ethyl acetate 1:1). The title compounds $\mathbf{4 g}$ (14.6 mg, $0.06 \mathrm{mmol}, 43 \%$ ), S15 (5.2 mg, $0.02 \mathrm{mmol}, 15 \%)$ and $\mathbf{S 1 6}(2.7 \mathrm{mg}, 0.01 \mathrm{mmol}, 8 \%)$ were obtained as colorless solids.

\section{Enantioselective [2+2] Photocycloaddition}

2(1H)-Quinolone (3.6 mg, $25.0 \mu \mathrm{mol}, 1.00$ eq.) and enantiomerically pure 3 (1.1 mg, $2.5 \mu \mathrm{mol}, 10 \mathrm{~mol} \%)$ were dissolved in $\alpha, \alpha, \alpha$-trifluorotoluene $(10 \mathrm{~mL}, c=2.5 \mathrm{mmol} / \mathrm{L})$. After degassing the solution, mesityl oxide (143 $\mu \mathrm{l}, 1.25 \mathrm{mmol}, 50.00$ eq.) was added and the mixture was irradiated at $419 \mathrm{~nm}$ for $8 \mathrm{~h}$ at $-25{ }^{\circ} \mathrm{C}(72 \%$ conversion $)$. After removing the solvent, purification was achieved by flash column chromatography (silica, pentane/ethyl acetate 1:1). The title compound $4 \mathrm{~g}(2.1 \mathrm{mg}, 8.5 \mu \mathrm{mol}, 34 \%$, $88 \% e e, 47 \%$ based on recovered starting material) was obtained as a colorless solid.

\section{Major regioisomer $(\mathbf{4 g})$ :}

M.p.: $151-153{ }^{\circ} \mathrm{C}$.

TLC: $R_{\mathrm{f}}=0.76$ (ethyl acetate, UV).

${ }^{1} \mathbf{H}-\mathbf{N M R}\left(500 \mathrm{MHz}, \mathrm{CDCl}_{3}, 303 \mathrm{~K}\right): \delta(\mathrm{ppm})=8.88$ (br. s, $\left.1 \mathrm{H}, \mathrm{NH}\right), 7.13$ (virt. td, ${ }^{3} \mathrm{~J} \approx$ $\left.{ }^{3} J=7.7 \mathrm{~Hz},{ }^{4} J=1.5 \mathrm{~Hz}, 1 \mathrm{H}, \mathrm{H}-6\right), 7.08\left(\mathrm{dd},{ }^{3} J=7.5 \mathrm{~Hz},{ }^{4} J=1.5 \mathrm{~Hz}, 1 \mathrm{H}, \mathrm{H}-8\right), 6.94$ (virt. td, $\left.{ }^{3} J \approx{ }^{3} J=7.5 \mathrm{~Hz},{ }^{4} J=1.2 \mathrm{~Hz}, 1 \mathrm{H}, \mathrm{H}-7\right), 6.77\left(\mathrm{dd},{ }^{3} J=7.9 \mathrm{~Hz},{ }^{4} J=1.2 \mathrm{~Hz}, 1 \mathrm{H}\right.$, H-5), 4.20 (virt. t, ${ }^{3} J \approx^{3} J=9.3 \mathrm{~Hz}, 1 \mathrm{H}, \mathrm{H}-8 \mathrm{~b}$ ), 3.09 (d, $\left.{ }^{3} J=8.7 \mathrm{~Hz}, 1 \mathrm{H}, \mathrm{H}-1\right), 2.88$ (d, ${ }^{3} J$ $=9.8 \mathrm{~Hz}, 1 \mathrm{H}, \mathrm{H}-2 \mathrm{a}), 2.07\left(\mathrm{~s}, 3 \mathrm{H}, \mathrm{COCH}_{3}\right), 1.41(\mathrm{~s}, 3 \mathrm{H}, \mathrm{Me}), 1.27(\mathrm{~s}, 3 \mathrm{H}, \mathrm{Me})$.

${ }^{13}$ C-NMR $\left(100 \mathrm{MHz}, \mathrm{CDCl}_{3}, 303 \mathrm{~K}\right): \delta(\mathrm{ppm})=206.7\left(\mathrm{~s}, \mathrm{COCH}_{3}\right), 169.9(\mathrm{~s}, \mathrm{C}-3)$, 136.7 (s, C-4a), 128.2 (d, C-8), 127.9 (d, C-6), 123.7 (d, C-7), 123.5 (s, C-8a), 115.6 (d, 
C-5), 64.4 (s, C-2), 45.2 (d, C-2a), 43.6 (d, C-1), 31.3 (d, C-8b), 30.4 (q, $\mathrm{COCH}_{3}$ ), 27.1 (q, Me), 25.6 (q, Me).

IR (ATR): $v\left(\mathrm{~cm}^{-1}\right)=1699,1660,1593,1493,1459,1389,1371,1352,1319,1301$, 1243, 1220, 1193, 1164, 1143, 875, 832, 750, 715, 676, 624, 602.

MS (EI, $70 \mathrm{eV}): m / z(\%)=243(10)\left[\mathrm{M}^{+}\right], 228(2)\left[\mathrm{M}^{+}-\mathrm{CH}_{3}\right], 200(16)\left[\mathrm{M}^{+}-\mathrm{C}_{2} \mathrm{H}_{3} \mathrm{O}\right]$, 186 (3), 173 (2), 158 (2), 145 (100) [ $\left.\mathrm{M}^{+}-\mathrm{C}_{6} \mathrm{H}_{10} \mathrm{O}\right], 117$ (16), 91 (7).

HRMS (EI) $\left(\mathrm{C}_{15} \mathrm{H}_{17} \mathrm{NO}_{2}\right)$ : calc.: 243.1254; found: 243.1255.

HPLC $\left(\right.$ AD-H, 250×4.6, $n$-hexane/iso-propanol = 90:10) $=e n t-4 \mathbf{g}, t_{\mathrm{R}}=16.7 \mathrm{~min} ; \mathbf{4 g}$, $30.1 \mathrm{~min})$.

Exo minor regioisomer $(\mathbf{S 1 5})$ :

M.p.: $176-178{ }^{\circ} \mathrm{C}$.

TLC: $R_{\mathrm{f}}=0.69$ (ethyl acetate, UV).

${ }^{1} \mathbf{H}-\mathbf{N M R}\left(500 \mathrm{MHz}, \mathrm{CDCl}_{3}, 303 \mathrm{~K}\right): \delta(\mathrm{ppm})=8.49$ (br. s, $\left.1 \mathrm{H}, \mathrm{NH}\right), 7.16$ (virt. td, ${ }^{3} \mathrm{~J} \approx$ $\left.{ }^{3} J=7.6 \mathrm{~Hz},{ }^{4} J=1.6 \mathrm{~Hz}, 1 \mathrm{H}, \mathrm{H}-6\right), 6.97$ (virt. td, ${ }^{3} J \approx{ }^{3} J=7.3 \mathrm{~Hz},{ }^{4} J=1.2 \mathrm{~Hz}, 1 \mathrm{H}, \mathrm{H}-7$ ), $6.90\left(\mathrm{dd},{ }^{3} J=7.2 \mathrm{~Hz},{ }^{4} J=1.6 \mathrm{~Hz}, 1 \mathrm{H}, \mathrm{H}-8\right), 6.74\left(\mathrm{dd},{ }^{3} J=7.8 \mathrm{~Hz},{ }^{4} J=1.2 \mathrm{~Hz}, 1 \mathrm{H}, \mathrm{H}-5\right)$, $3.80\left(\mathrm{dd},{ }^{3} \mathrm{~J}=10.4 \mathrm{~Hz},{ }^{3} \mathrm{~J}=5.3 \mathrm{~Hz}, 1 \mathrm{H}, \mathrm{H}-2 \mathrm{a}\right), 3.46\left(\mathrm{~d},{ }^{3} \mathrm{~J}=10.4 \mathrm{~Hz}, 1 \mathrm{H}, \mathrm{H}-2\right), 3.35$ (d, $\left.{ }^{3} J=5.3 \mathrm{~Hz}, 1 \mathrm{H}, \mathrm{H}-8 \mathrm{~b}\right), 2.17$ (s, 3H, COCH$\left.{ }_{3}\right), 1.17$ (s, 3H, Me), 1.06 (s, 3H, Me).

${ }^{13} \mathbf{C}-\mathbf{N M R}\left(100 \mathrm{MHz}, \mathrm{CDCl}_{3}, 303 \mathrm{~K}\right): \delta(\mathrm{ppm})=205.9\left(\mathrm{~s}, \mathrm{COCH}_{3}\right), 171.9(\mathrm{~s}, \mathrm{C}-3)$, 137.2 (s, C-4a), 129.2 (d, C-8), 128.2 (d, C-6), 123.4 (d, C-7), 120.3 (s, C-8a), 115.8 (d, C-5), 61.0 (d, C-8b), 44.2 (d, C-2), 43.9 (s, C-1), 33.2 (d, C-2a), 31.1 (q, $\mathrm{COCH}_{3}$ ), 26.4 (q, Me), 25.8 (q, Me).

MS (EI, $70 \mathrm{eV}): m / z(\%)=243(1)\left[\mathrm{M}^{+}\right], 228(1)\left[\mathrm{M}^{+}-\mathrm{CH}_{3}\right], 200(4)\left[\mathrm{M}^{+}-\mathrm{C}_{2} \mathrm{H}_{3} \mathrm{O}\right], 158$ (2), 145 (100) $\left[\mathrm{M}^{+}-\mathrm{C}_{6} \mathrm{H}_{10} \mathrm{O}\right], 130$ (4), 117 (15), 91 (4).

HRMS (EI) $\left(\mathrm{C}_{15} \mathrm{H}_{17} \mathrm{NO}_{2}\right)$ : calc.: 243.1254; found: 243.1252 .

Endo minor regioisomer (S16):

TLC: $R_{\mathrm{f}}=0.58$ (ethyl acetate, UV).

${ }^{1}$ H-NMR $\left(500 \mathrm{MHz}, \mathrm{CDCl}_{3}, 303 \mathrm{~K}\right): \delta$ (ppm) = 7.67 (br. s, $\left.1 \mathrm{H}, \mathrm{NH}\right), 7.15$ (virt. td, ${ }^{3} J \approx$ $\left.{ }^{3} J=7.6 \mathrm{~Hz},{ }^{4} J=1.6 \mathrm{~Hz}, 1 \mathrm{H}, \mathrm{H}-6\right), 6.95$ (virt. td, ${ }^{3} J \approx{ }^{3} J=7.4 \mathrm{~Hz},{ }^{4} J=1.2 \mathrm{~Hz}, 1 \mathrm{H}, \mathrm{H}-7$ ), $6.91\left(\mathrm{dd},{ }^{3} J=7.6 \mathrm{~Hz},{ }^{4} J=1.6 \mathrm{~Hz}, 1 \mathrm{H}, \mathrm{H}-8\right), 6.67\left(\mathrm{dd},{ }^{3} J=7.7 \mathrm{~Hz},{ }^{4} J=1.2 \mathrm{~Hz}, 1 \mathrm{H}, \mathrm{H}-5\right)$, 3.65 (virt. t, $\left.{ }^{3} J \approx{ }^{3} J=10.1 \mathrm{~Hz}, 1 \mathrm{H}, \mathrm{H}-2 \mathrm{a}\right), 3.54$ (d, $\left.{ }^{3} \mathrm{~J}=10.0 \mathrm{~Hz}, 1 \mathrm{H}, \mathrm{H}-2\right), 3.43\left(\mathrm{~d},{ }^{3} J=\right.$ $10.2 \mathrm{~Hz}, 1 \mathrm{H}, \mathrm{H}-8 \mathrm{~b}), 2.20$ (s, 3H, $\left.\mathrm{COCH}_{3}\right), 1.40$ (s, 3H, Me), 0.89 (s, 3H, Me).

${ }^{13}$ C-NMR $\left(100 \mathrm{MHz}, \mathrm{CDCl}_{3}, 303 \mathrm{~K}\right): \delta(\mathrm{ppm})=206.9\left(\mathrm{~s}, \mathrm{COCH}_{3}\right), 169.6(\mathrm{~s}, \mathrm{C}-3)$, 137.4 (s, C-4a), 129.0 (d, C-8), 128.1 (d, C-6), 123.0 (d, C-7), 120.1 (s, C-8a), 115.5 (d, C-5), 58.0 (d, C-2), 44.7 (d, C-8b), 44.4 (s, C-1), 36.1 (d, C-2a), 32.4 (q, Me), 31.6 (q, $\left.\mathrm{COCH}_{3}\right), 20.0$ (q, Me).

MS (EI, $70 \mathrm{eV}): m / z(\%)=243(1)\left[\mathrm{M}^{+}\right], 228(1)\left[\mathrm{M}^{+}-\mathrm{CH}_{3}\right], 200(4)\left[\mathrm{M}^{+}-\mathrm{C}_{2} \mathrm{H}_{3} \mathrm{O}\right], 158$ (2), 145 (100) [ $\left.\mathrm{M}^{+}-\mathrm{C}_{6} \mathrm{H}_{10} \mathrm{O}\right], 130$ (4), 117 (15), 91 (4). 


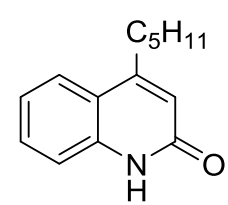<smiles>C=CC(C)=O</smiles>
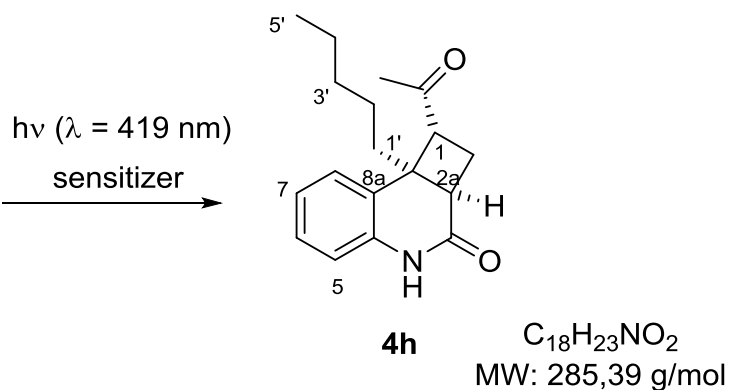

\section{Racemic [2+2] Photocycloaddition}

4-Pentylquinolin-2-(1H)-one ${ }^{[J . ~ O r g . ~ C h e m . ~ 1969, ~ 34, ~ 3263-3268 .] ~}(30.1 \mathrm{mg}, 0.14 \mathrm{mmol}, 1.00$ eq.) and thioxanthen-9-one $(15 \mathrm{mg}, 0.07 \mathrm{mmol}, 0.50 \mathrm{eq}$.) were dissolved in acetonitrile $(10 \mathrm{~mL}, c=14 \mathrm{mmol} / \mathrm{L})$. After degassing the solution, 3-buten-2-one (115 $\mu \mathrm{l}, 1.4$ mmol, 10.00 eq.) was added and the mixture was irradiated at $419 \mathrm{~nm}$ for $10 \mathrm{~h}$ at room temperature ( $85 \%$ conversion). After removing the solvent, purification was achieved by flash column chromatography ( silica, pentane/ethyl acetate 2:1). The title compound 4h (31.5 mg, $0.11 \mathrm{mmol}, 79 \%)$ was obtained as a colorless solid.

\section{Enantioselective [2+2] Photocycloaddition}

4-Pentylquinolin-2-( $1 H)$-one (5.4 mg, $25.0 \mu \mathrm{mol}, 1.00$ eq.) and enantiomerically pure 3 $(1.1 \mathrm{mg}, 2.5 \mu \mathrm{mol}, 10 \mathrm{~mol} \%)$ were dissolved in $\alpha, \alpha, \alpha$-trifluorotoluene $(10 \mathrm{~mL}, c=$ $2.5 \mathrm{mmol} / \mathrm{L})$. After degassing the solution, 3-buten-2-one (104 $\mu \mathrm{l}, 1.25 \mathrm{mmol}, 50.00$ eq.) was added and the mixture was irradiated at $419 \mathrm{~nm}$ for $18 \mathrm{~h}$ at $-25^{\circ} \mathrm{C}$. After removing the solvent, purification was achieved by flash column chromatography (silica, pentane/ethyl acetate 2:1). The title compound $\mathbf{4 h}$ (6.7 mg, $23.0 \mu \mathrm{mol}, 94 \%, 90$ $\%$ ee) was obtained as a colorless solid.

M.p.: $88-90{ }^{\circ} \mathrm{C}$.

TLC: $R_{\mathrm{f}}=0.82$ (ethyl acetate, UV).

${ }^{1}$ H-NMR $\left(500 \mathrm{MHz}, \mathrm{CDCl}_{3}, 303 \mathrm{~K}\right): \delta(\mathrm{ppm})=9.27$ (br. s, $\left.1 \mathrm{H}, \mathrm{NH}\right), 7.27-7.17(\mathrm{~m}$, $2 \mathrm{H}, \mathrm{H}-6, \mathrm{H}-8$ ), 7.09 (virt. td, ${ }^{3} J \approx{ }^{3} J=7.6 \mathrm{~Hz},{ }^{4} J=1.2 \mathrm{~Hz}, 1 \mathrm{H}, \mathrm{H}-7$ ), 6.86 (dd, ${ }^{3} J=7.9$ $\mathrm{Hz},{ }^{4} J=1.2 \mathrm{~Hz}, 1 \mathrm{H}, \mathrm{H}-5$ ), 3.44 (virt. $\mathrm{t},{ }^{3} J \approx{ }^{3} J=7.3 \mathrm{~Hz}, 1 \mathrm{H}, \mathrm{H}-1$ ), 3.05 (dd, ${ }^{3} J=10.5$ $\left.\mathrm{Hz},{ }^{3} J=6.4 \mathrm{~Hz}, 1 \mathrm{H}, \mathrm{H}-2 \mathrm{a}\right), 2.92\left(\mathrm{ddd},{ }^{2} J=12.1 \mathrm{~Hz},{ }^{3} J=10.5 \mathrm{~Hz},{ }^{3} J=6.4 \mathrm{~Hz}, 1 \mathrm{H}, \mathrm{H}-2\right)$, $2.24\left(\mathrm{ddd},{ }^{2} J=12.1 \mathrm{~Hz},{ }^{3} J=8.8 \mathrm{~Hz},{ }^{3} J=6.4 \mathrm{~Hz}, 1 \mathrm{H}, \mathrm{H}-2\right), 2.19(\mathrm{~s}, 3 \mathrm{H}, \mathrm{Me}), 1.81\left(\mathrm{dt},{ }^{2} J\right.$ $=13.4 \mathrm{~Hz},{ }^{3} J=4.3 \mathrm{~Hz}, 1 \mathrm{H}, \mathrm{H}-1$ '), $1.66\left(\mathrm{dt},{ }^{2} J=13.4 \mathrm{~Hz},{ }^{3} J=3.5 \mathrm{~Hz}, 1 \mathrm{H}, \mathrm{H}-1^{\prime}\right), 1.21-$ 1.04 (m, 6H, H-2', H-3', H-4'), 0.79 (t, $\left.{ }^{3} J=6.7 \mathrm{~Hz}, 3 \mathrm{H}, \mathrm{H}-5^{\prime}\right)$.

${ }^{13} \mathrm{C}-\mathrm{NMR}\left(100 \mathrm{MHz}, \mathrm{CDCl}_{3}, 303 \mathrm{~K}\right): \delta(\mathrm{ppm})=207.1(\mathrm{~s}, \mathrm{COMe}), 172.2(\mathrm{~s}, \mathrm{C}-3), 136.9$ (s, C-4a), 128.4 (d, C-8), 126.8 (s, C-8a), 126.2 (d, C-6), 124.2 (d, C-7), 116.4 (d, C-5), 56.8 (d, C-1), 48.3 (s, C-8b), 40.1 (d, C-2a), 36.0 (t, C-1'), 32.0 (t, C-2'), 31.1 (q, Me), 24.2 (t, C-2), 23.2 (t, C-3'), 22.5 (t, C-4'), 14.1 (q, C-5').

IR (ATR): $v\left(\mathrm{~cm}^{-1}\right)=1749,1734,1716,1697,1671,1654,1637,1593,1558,1542$, 1522, 1508, 1490, 1473, 1457, 1437, 1457, 1438, 1418, 1388, 1362, 1339, 1168, 756, $728,707,662,620,603$. 
MS (EI, $70 \mathrm{eV}): m / z(\%)=285(1)\left[\mathrm{M}^{+}\right], 270(1)\left[\mathrm{M}^{+}-\mathrm{CH}_{3}\right], 256(1)\left[\mathrm{M}^{+}-\mathrm{C}_{2} \mathrm{H}_{5}\right], 242$ (26) $\left[\mathrm{M}^{+}-\mathrm{C}_{3} \mathrm{H}_{7}\right], 229$ (9) $\left[\mathrm{M}^{+}-\mathrm{C}_{4} \mathrm{H}_{9}\right], 215$ (24) $\left[\mathrm{M}^{+}-\mathrm{C}_{5} \mathrm{H}_{11}\right], 200$ (1) $\left[\mathrm{M}^{+}-\mathrm{C}_{6} \mathrm{H}_{14}\right], 186$ (6), $172(9)\left[\mathrm{M}^{+}-\mathrm{C}_{7} \mathrm{H}_{14} \mathrm{O}\right], 159(100)\left[\mathrm{M}^{+}-\mathrm{C}_{8} \mathrm{H}_{15} \mathrm{O}\right], 146(4)\left[\mathrm{M}^{+}-\mathrm{C}_{9} \mathrm{H}_{17} \mathrm{O}\right], 130$ (27).

HRMS (EI) $\left(\mathrm{C}_{18} \mathrm{H}_{23} \mathrm{NO}_{2}\right)$ : calc.: 285.1723; found: 285.1722 .

HPLC $(\mathrm{OJ}-\mathrm{H}, 250 \times 4.6, n$-hexane $/$ iso -propanol $=95: 5)=$ ent $-\mathbf{4 h}, t_{\mathrm{R}}=22.5 \mathrm{~min} ; \mathbf{4 h}, 25.0$ $\min )$. 

one (4i)

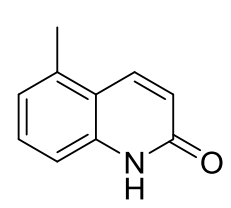

S3

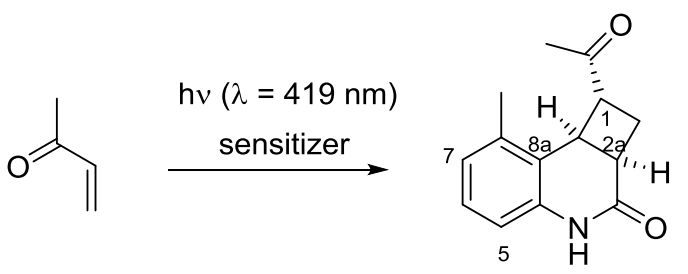

$4 \mathbf{i} \quad \mathrm{C}_{14} \mathrm{H}_{15} \mathrm{NO}_{2}$ MW: $229,28 \mathrm{~g} / \mathrm{mol}$

\section{Racemic [2+2] Photocycloaddition}

5-Methylquinolin-2(1H)-one (S3) $(22.0 \mathrm{mg}, 0.14 \mathrm{mmol}, 1.00 \mathrm{eq}$.$) and thioxanthen-9-$ one $(15 \mathrm{mg}, 0.07 \mathrm{mmol}, 0.50$ eq.) were dissolved in acetonitrile $(10 \mathrm{~mL}$, $c=14 \mathrm{mmol} / \mathrm{L})$. After degassing the solution, 3-buten-2-one (115 $\mu \mathrm{l}, 1.4 \mathrm{mmol}$, 10.00 eq.) was added and the mixture was irradiated at $419 \mathrm{~nm}$ for $5 \mathrm{~h}$ at room temperature. After removing the solvent, purification was achieved by flash column chromatography (silica, pentane/ethyl acetate $1: 1)$. The title compound $\mathbf{4 i}(17.7 \mathrm{mg}$, $0.08 \mathrm{mmol}, 56 \%$ ) was obtained as a colorless solid.

\section{Enantioselective [2+2] Photocycloaddition}

5-Methylquinolin-2(1H)-one $(4.0 \mathrm{mg}, 25.0 \mu \mathrm{mol}, 1.00 \mathrm{eq}$.) and enantiomerically pure 3 $(1.1 \mathrm{mg}, 2.5 \mu \mathrm{mol}, 10 \mathrm{~mol} \%)$ were dissolved in $\alpha, \alpha, \alpha$-trifluorotoluene $(10 \mathrm{~mL}, c=$ $2.5 \mathrm{mmol} / \mathrm{L})$. After degassing the solution, 3-buten-2-one (104 $\mu \mathrm{l}, 1.25 \mathrm{mmol}, 50.00$ eq.) was added and the mixture was irradiated at $419 \mathrm{~nm}$ for $10 \mathrm{~h}$ at $-25{ }^{\circ} \mathrm{C}(73 \%$ conversion). After removing the solvent, purification was achieved by flash column chromatography (silica, pentane/ethyl acetate 1:1). The title compound $\mathbf{4 i}$ ( $2.0 \mathrm{mg}, 8.8$ $\mu \mathrm{mol}, 35 \%, 91 \% e e, 48 \%$ based on recovered starting material) was obtained as a colorless solid.

M.p.: $162-164{ }^{\circ} \mathrm{C}$.

TLC: $R_{\mathrm{f}}=0.58$ (ethyl acetate, UV).

${ }^{1} \mathbf{H}$-NMR $\left(500 \mathrm{MHz}, \mathrm{CDCl}_{3}, 303 \mathrm{~K}\right): \delta(\mathrm{ppm})=9.02$ (br. s, $\left.1 \mathrm{H}, \mathrm{NH}\right), 7.07$ (virt. t, ${ }^{3} \mathrm{~J} \approx$ $\left.{ }^{3} J=7.7 \mathrm{~Hz}, 1 \mathrm{H}, \mathrm{H}-6\right), 6.83\left(\mathrm{~d},{ }^{3} J=7.5 \mathrm{~Hz}, 1 \mathrm{H}, \mathrm{H}-7\right), 6.66\left(\mathrm{~d},{ }^{3} J=7.9 \mathrm{~Hz}, 1 \mathrm{H}, \mathrm{H}-5\right)$, 4.22 (virt. $\mathrm{t},{ }^{3} J \approx{ }^{3} J=8.6 \mathrm{~Hz}, 1 \mathrm{H}, \mathrm{H}-8 \mathrm{~b}$ ), 3.51 (virt. q, ${ }^{3} J \approx{ }^{3} J \approx{ }^{3} J=9.0 \mathrm{~Hz}, 1 \mathrm{H}, \mathrm{H}-1$ ), 3.28 (virt. td, ${ }^{3} J \approx{ }^{3} J=9.1 \mathrm{~Hz},{ }^{3} J=4.2 \mathrm{~Hz}, 1 \mathrm{H}, \mathrm{H}-2 \mathrm{a}$ ), $2.77-2.60(\mathrm{~m}, 2 \mathrm{H}, \mathrm{H}-2), 2.19$ (s, 3H, Me), 2.12 (s, 3H, $\left.\mathrm{COCH}_{3}\right)$.

${ }^{13} \mathrm{C}-\mathrm{NMR}\left(100 \mathrm{MHz}, \mathrm{CDCl}_{3}, 303 \mathrm{~K}\right): \delta(\mathrm{ppm})=207.5\left(\mathrm{~s}, \mathrm{COCH}_{3}\right), 172.5(\mathrm{~s}, \mathrm{C}-3)$, 137.1 (s, C-4a), 136.8 (s, C-8), 128.2 (d, C-6), 125.8 (d, C-7), 121.3 (s, C-8a), 113.9 (d, C-5), 51.9 (d, C-1), 35.7 (d, C-8b), 34.3 (d, C-2a), 30.1 (t, C-2), 28.7 (q, $\left.\mathrm{COCH}_{3}\right), 19.3$ (q, Me).

IR (ATR): $v\left(\mathrm{~cm}^{-1}\right)=1699,1667,1590,1558,1542,1521,1507,1489,1474,1457$, 1437, 1418, 1396, 1362, 1340, 1314, 1233, 1200, 1177, 1162, 887, 852, 828, 804, 789, $755,704,668,620$.

MS (EI, $70 \mathrm{eV}): m / z(\%)=229(7)\left[\mathrm{M}^{+}\right], 186(56)\left[\mathrm{M}^{+}-\mathrm{C}_{2} \mathrm{H}_{3} \mathrm{O}\right], 168$ (4), 159 (100) $\left[\mathrm{M}^{+}-\mathrm{C}_{4} \mathrm{H}_{6} \mathrm{O}\right], 141$ (2), 130 (35), 115 (4), 103 (5), 77 (8) [ $\left.\mathrm{C}_{6} \mathrm{H}_{5}\right]$. 
HRMS (ESI) $\left(\mathrm{C}_{14} \mathrm{H}_{16} \mathrm{NO}_{2}{ }^{+}\right)$: calc.: 230.1176; found: 230.1174 .

Chiral GC $\left[60{ }^{\circ} \mathrm{C}(1 \mathrm{~min}), 15^{\circ} \mathrm{C} / \mathrm{min} \rightarrow 200{ }^{\circ} \mathrm{C}(30 \mathrm{~min})\right]=$ ent $-4 \mathbf{i}, t_{\mathrm{R}}=34.1 \mathrm{~min} ; \mathbf{4 i}, 34.8$ $\min )$. 
$(1 R, 2 \mathrm{a} R, 8 \mathrm{bS})$-1-Acetyl-7-bromo-2,2a,4,8b-tetrahydrocyclobuta[c]quinolin-3(1H)one $(\mathbf{4 j})$

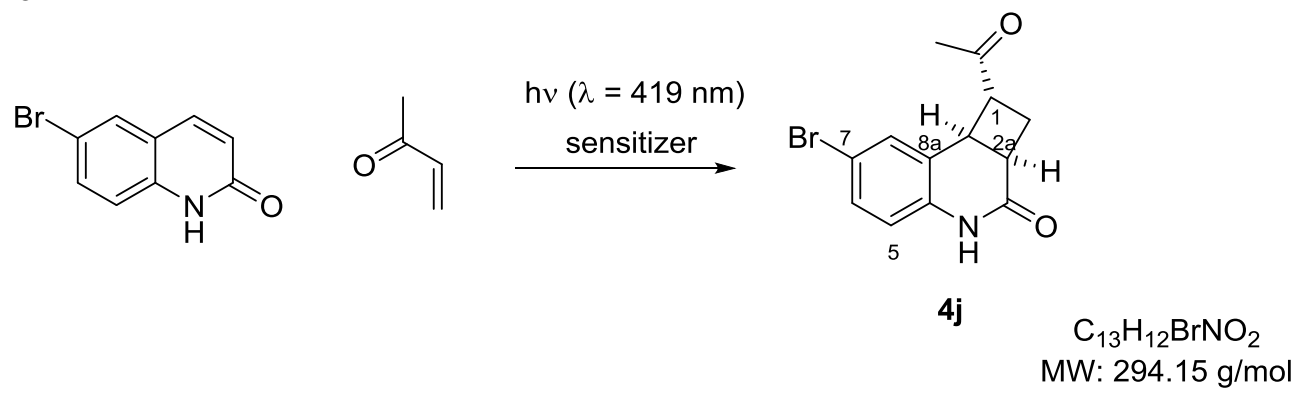

Racemic [2+2] Photocycloaddition

6-Bromoquinolin-2(1H)-one (31.4 mg, $0.14 \mathrm{mmol}, 1.00 \mathrm{eq}$.$) and thioxanthen-9-one$ (15 mg, $0.07 \mathrm{mmol}, 0.50$ eq.) were dissolved in acetonitrile $(10 \mathrm{~mL}, c=14 \mathrm{mmol} / \mathrm{L})$. After degassing the solution, 3-buten-2-one (115 $\mu \mathrm{l}, 1.4 \mathrm{mmol}, 10.00 \mathrm{eq}$.) was added and the mixture was irradiated at $419 \mathrm{~nm}$ for $9 \mathrm{~h}$ at room temperature (78 $\%$ conversion). After removing the solvent, purification was achieved by flash column chromatography (silica, pentane/ethyl acetate 1:1). The title compound $\mathbf{4 j}$ (21.9 $\mathrm{mg}$, $0.07 \mathrm{mmol}, 54 \%$ ) was obtained as a colorless solid.

\section{Enantioselective [2+2] Photocycloaddition}

6-Bromoquinolin-2(1H)-one (5.6 mg, $25.0 \mu \mathrm{mol}, 1.00 \mathrm{eq}$.) and enantiomerically pure 3 (1.1 mg, $2.5 \mu \mathrm{mol}, 10 \mathrm{~mol} \%)$ were dissolved in $\alpha, \alpha, \alpha$-trifluorotoluene $(10 \mathrm{~mL}, c=2.5$ $\mathrm{mmol} / \mathrm{L}$ ). After degassing the solution, 3-buten-2-one (104 $\mu \mathrm{l}, 1.25 \mathrm{mmol}, 50.00 \mathrm{eq}$.) was added and the mixture was irradiated at $419 \mathrm{~nm}$ for $9 \mathrm{~h}$ at $-25{ }^{\circ} \mathrm{C}(61 \%$ conversion). After removing the solvent, purification was achieved by flash column chromatography (silica, pentane/ethyl acetate $1: 1$ ). The title compound $\mathbf{4 j}$ ( $2.7 \mathrm{mg}, 9.0$ $\mu \mathrm{mol}, 36 \%, 94 \% e e, 59 \%$ based on recovered starting material) was obtained as a colorless solid.

M.p.: $167-169^{\circ} \mathrm{C}$.

TLC: $R_{\mathrm{f}}=0.68$ (ethyl acetate, UV).

${ }^{1} \mathbf{H}-\mathbf{N M R}\left(500 \mathrm{MHz}, \mathrm{CDCl}_{3}, 303 \mathrm{~K}\right): \delta(\mathrm{ppm})=9.32$ (br. s, $\left.1 \mathrm{H}, \mathrm{NH}\right), 7.28\left(\mathrm{dd},{ }^{3} J=8.4\right.$ $\left.\mathrm{Hz},{ }^{4} J=2.2 \mathrm{~Hz}, 1 \mathrm{H}, \mathrm{H}-6\right), 7.24\left(\mathrm{~d},{ }^{4} \mathrm{~J}=2.2 \mathrm{~Hz}, 1 \mathrm{H}, \mathrm{H}-8\right), 6.72\left(\mathrm{~d},{ }^{3} J=8.4 \mathrm{~Hz}, 1 \mathrm{H}, \mathrm{H}-5\right)$, 4.00 (virt. $\mathrm{t},{ }^{3} J \approx{ }^{3} J=8.9 \mathrm{~Hz}, 1 \mathrm{H}, \mathrm{H}-8 \mathrm{~b}$ ), 3.44 (virt. $\mathrm{td},{ }^{3} J \approx{ }^{3} J=10.1 \mathrm{~Hz},{ }^{3} J=8.2 \mathrm{~Hz}$, $1 \mathrm{H}, \mathrm{H}-1$ ), 3.22 (virt. td, ${ }^{3} J \approx{ }^{3} J=9.4 \mathrm{~Hz},{ }^{3} J=2.9 \mathrm{~Hz}, 1 \mathrm{H}, \mathrm{H}-2 \mathrm{a}$ ), 2.74 (virt. dt, ${ }^{2} J=12.0$ $\left.\mathrm{Hz},{ }^{3} J \approx{ }^{3} J=9.7 \mathrm{~Hz}, 1 \mathrm{H}, \mathrm{H}-2\right), 2.64\left(\mathrm{ddd},{ }^{2} J=12.0 \mathrm{~Hz},{ }^{3} J=9.3 \mathrm{~Hz},{ }^{3} J=2.9 \mathrm{~Hz}, 1 \mathrm{H}, \mathrm{H}-\right.$ 2), $2.11\left(\mathrm{~s}, 3 \mathrm{H}, \mathrm{COCH}_{3}\right)$.

${ }^{13}$ C-NMR $\left(100 \mathrm{MHz}, \mathrm{CDCl}_{3}, 303 \mathrm{~K}\right): \delta(\mathrm{ppm})=206.6\left(\mathrm{~s}, \mathrm{COCH}_{3}\right), 172.6(\mathrm{~s}, \mathrm{C}-3)$, 136.1 (s, C-4a), 131.3 (d, C-6), 130.8 (d, C-8), 124.9 (s, C-8a), 117.5 (d, C-5), 116.1 (s, C-7), 52.6 (d, C-1), 38.2 (d, C-8b), 34.0 (d, C-2a), 29.3 (t, C-2), 27.9 (q, $\mathrm{COCH}_{3}$ ).

IR (ATR): $v\left(\mathrm{~cm}^{-1}\right)=1698,1669,1586,1489,1446,1412,1377,1362,1342,1308$, 1281, 1248, 1197, 1180, 1155, 1132, 1076, 1045, 986, 939, 906, 881, 851, 822, 807, $755,725,701,626$. 
MS (EI, $70 \mathrm{eV}): m / z(\%)=295(8)\left[\mathrm{M}^{+}\left({ }^{81} \mathrm{Br}\right)\right], 293(9)\left[\mathrm{M}^{+}\left({ }^{79} \mathrm{Br}\right)\right], 252(42)\left[\mathrm{M}^{+}\right.$ $\left.\left({ }^{81} \mathrm{Br}\right)-\mathrm{C}_{2} \mathrm{H}_{3} \mathrm{O}\right], 250(42)\left[\mathrm{M}^{+}\left({ }^{79} \mathrm{Br}\right)-\mathrm{C}_{2} \mathrm{H}_{3} \mathrm{O}\right], 225(93)\left[\mathrm{M}^{+}\left({ }^{81} \mathrm{Br}\right)-\mathrm{C}_{4} \mathrm{H}_{6} \mathrm{O}\right], 223$ (100) $\left[\mathrm{M}^{+}\left({ }^{79} \mathrm{Br}\right)-\mathrm{C}_{4} \mathrm{H}_{6} \mathrm{O}\right], 197$ (19), 195 (21), 116 (27), 89 (21).

HRMS (EI) $\left(\mathrm{C}_{13} \mathrm{H}_{12} \mathrm{BrNO}_{2}\right)$ : calc.: 295.0025; found: 295.0004 .

HPLC (AD-H, 250×4.6, $n$-hexane/iso-propanol $=90: 10)=\mathbf{4 j}$, $t_{\mathrm{R}}=17.7 \mathrm{~min} ;$ ent-4j, $21.3 \mathrm{~min})$.

Specific rotation $[\alpha]_{\mathrm{D}}^{20}=-54.7(c=0.28, \mathrm{MeOH})[>99 \% e e]$. 
<smiles>Cc1ccc2[nH]c(=O)ccc2c1</smiles>

56

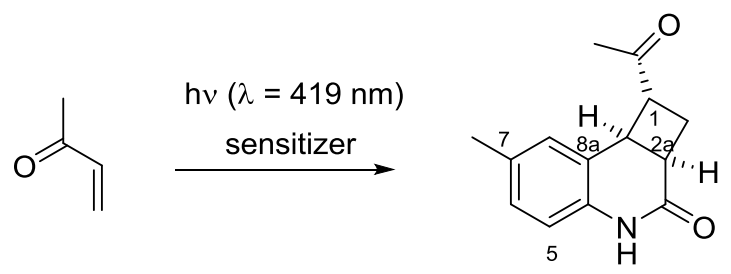

$4 \mathbf{k}$
$\mathrm{C}_{14} \mathrm{H}_{15} \mathrm{NO}_{2}$ MW: $229.28 \mathrm{~g} / \mathrm{mol}$

\section{Racemic [2+2] Photocycloaddition}

6-Methylquinolin-2(1H)-one (22 $\mathrm{mg}, 0.14 \mathrm{mmol}, 1.00 \mathrm{eq}$.$) and thioxanthen-9-one$ (15 mg, $0.07 \mathrm{mmol}, 0.50$ eq.) were dissolved in acetonitrile $(10 \mathrm{~mL}, c=14 \mathrm{mmol} / \mathrm{L})$. After degassing the solution, 3-buten-2-one (115 $\mu \mathrm{l}, 1.4 \mathrm{mmol}, 10.00 \mathrm{eq}$.) was added and the mixture was irradiated at $419 \mathrm{~nm}$ for $9 \mathrm{~h}$ at room temperature $(61 \%$ conversion). After removing the solvent, purification was achieved by flash column chromatography (silica, pentane/ethyl acetate 1:1). The title compound $4 \mathbf{k}$ (14.2 $\mathrm{mg}$, $0.06 \mathrm{mmol}, 45 \%$ ) was obtained as a colorless solid. A second fraction was collected containing a non-characterized cyclobutane $(0.9 \mathrm{mg}, 4 \mu \mathrm{mol}, 4 \%, R \mathrm{f}=0.72$ (ethyl acetate, UV)).

\section{Enantioselective [2+2] Photocycloaddition}

6-Methylquinolin-2(1H)-one (4.0 mg, $25.0 \mu \mathrm{mol}, 1.00 \mathrm{eq}$.) and enantiomerically pure 3 $(1.1 \mathrm{mg}, 2.5 \mu \mathrm{mol}, 10 \mathrm{~mol} \%)$ were dissolved in $\alpha, \alpha, \alpha$-trifluorotoluene $(10 \mathrm{~mL}, c=$ $2.5 \mathrm{mmol} / \mathrm{L})$. After degassing the solution, 3-buten-2-one (104 $\mu \mathrm{l}, 1.25 \mathrm{mmol}, 50.00$ eq.) was added and the mixture was irradiated at $419 \mathrm{~nm}$ for $7 \mathrm{~h}$ at $-25{ }^{\circ} \mathrm{C}(55 \%$ conversion). After removing the solvent, purification was achieved by flash column chromatography (silica, pentane/ethyl acetate $2: 1)$. The title compound $4 \mathbf{k}(2.2 \mathrm{mg}, 9.8$ $\mu$ mol, $39 \%, 85 \% e e, 71 \%$ based on recovered starting material) was obtained as a colorless solid.

M.p.: $120-122^{\circ} \mathrm{C}$.

TLC: $R_{\mathrm{f}}=0.62$ (ethyl acetate, UV).

${ }^{1}$ H-NMR $\left(500 \mathrm{MHz}, \mathrm{CDCl}_{3}, 303 \mathrm{~K}\right): \delta(\mathrm{ppm})=8.60$ (br. s, $\left.1 \mathrm{H}, \mathrm{NH}\right), 6.98\left(\mathrm{~d},{ }^{3} J=7.8\right.$ $\mathrm{Hz}, 1 \mathrm{H}, \mathrm{H}-6), 6.91$ (s, $1 \mathrm{H}, \mathrm{H}-8), 6.68$ (d, $\left.{ }^{3} J=7.8 \mathrm{~Hz}, 1 \mathrm{H}, \mathrm{H}-5\right), 3.95$ (virt. t, ${ }^{3} J \approx^{3} J=$ $8.8 \mathrm{~Hz}, 1 \mathrm{H}, \mathrm{H}-8 \mathrm{~b}$ ), 3.44 (virt. td, ${ }^{3} J \approx^{3} J=10.6 \mathrm{~Hz},{ }^{3} J=8.5 \mathrm{~Hz}, 1 \mathrm{H}, \mathrm{H}-1$ ), 3.21 (virt. td, ${ }^{3} J \approx{ }^{3} J=9.5 \mathrm{~Hz},{ }^{3} J=3.2 \mathrm{~Hz}, 1 \mathrm{H}, \mathrm{H}-2 \mathrm{a}$ ), 2.75 (virt. dt, ${ }^{2} J=12.2 \mathrm{~Hz},{ }^{3} J \approx{ }^{3} J=9.7 \mathrm{~Hz}$, $1 \mathrm{H}, \mathrm{H}-2), 2.60$ (ddd, $\left.{ }^{2} J=12.2 \mathrm{~Hz},{ }^{3} J=9.5 \mathrm{~Hz},{ }^{3} J=3.2 \mathrm{~Hz}, 1 \mathrm{H}, \mathrm{H}-2\right), 2.27$ (s, 3H, Me), $2.11\left(\mathrm{~s}, 3 \mathrm{H}, \mathrm{COCH}_{3}\right)$.

${ }^{13} \mathrm{C}-\mathrm{NMR}\left(100 \mathrm{MHz}, \mathrm{CDCl}_{3}, 303 \mathrm{~K}\right): \delta(\mathrm{ppm})=207.2\left(\mathrm{~s}, \mathrm{COCH}_{3}\right), 172.4(\mathrm{~s}, \mathrm{C}-3)$, 134.4 (s, C-4a), 133.5 (s, C-7), 128.9 (d, C-6), 128.5 (d, C-8), 122.8 (s, C-8a), 115.7 (d, C-5), 52.9 (d, C-1), 39.0 (d, C-8b), 34.3 (d, C-2a), 29.0 (t, C-2), 28.1 (q, $\mathrm{COCH}_{3}$ ), 20.8 (q, Me). 
IR (ATR): $v\left(\mathrm{~cm}^{-1}\right)=1715,1698,1666,1617,1602,1507,1489,1457,1447,1417$, 1389, 1362, 1311, 1248, 1233, 1193, 1175, 1151, 954, 842, 818, 796, 736, 729, 703, 620.

MS (EI, $70 \mathrm{eV}): m / z(\%)=229(10)\left[\mathrm{M}^{+}\right], 186(47)\left[\mathrm{M}^{+}-\mathrm{C}_{2} \mathrm{H}_{3} \mathrm{O}\right], 159$ (100) $\left[\mathrm{M}^{+}-\right.$ $\left.\mathrm{C}_{4} \mathrm{H}_{6} \mathrm{O}\right], 130$ (22), 115 (3), 103 (4), 77 (4).

HRMS (EI) $\left(\mathrm{C}_{14} \mathrm{H}_{15} \mathrm{NO}_{2}\right)$ : calc.: 229.1097; found: 229.1097 .

HPLC $($ AD-H, 250×4.6, $n$-hexane/iso-propanol $=95: 5)=4 \mathbf{k}, t_{\mathrm{R}}=35.7 \mathrm{~min} ;$ ent-4k, $44.3 \mathrm{~min})$. 


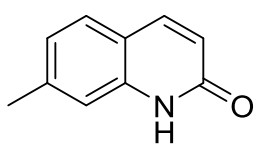

S8

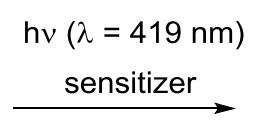

4!<smiles>CC(=O)[C@H]1C[C@H]2c3ccc(C)cc3NC(=O)[C@H]12</smiles>

S17

$\mathrm{C}_{14} \mathrm{H}_{15} \mathrm{NO}_{2}$

MW: $229.28 \mathrm{~g} / \mathrm{mol}$

\section{Racemic [2+2] Photocycloaddition}

7-Methylquinolin-2(1H)-one (22 mg, $0.14 \mathrm{mmol}, 1.00$ eq.) and thioxanthen-9-one (15 mg, $0.07 \mathrm{mmol}, 0.50$ eq.) were dissolved in acetonitrile $(10 \mathrm{~mL}, c=14 \mathrm{mmol} / \mathrm{L})$. After degassing the solution, 3-buten-2-one (115 $\mu \mathrm{l}, 1.4 \mathrm{mmol}, 10.00$ eq.) was added and the mixture was irradiated at $419 \mathrm{~nm}$ for $6 \mathrm{~h}$ at room temperature. After removing the solvent, purification was achieved by flash column chromatography (silica, pentane/ethyl acetate 1:1). The title compounds $\mathbf{4 l}(21.4 \mathrm{mg}, 0.09 \mathrm{mmol}, 68 \%)$ and $\mathbf{S 1 7}$ (4.4 mg, $0.02 \mathrm{mmol}, 14 \%$ ) were obtained as colorless solids.

\section{Enantioselective [2+2] Photocycloaddition}

7-Methylquinolin-2(1H)-one (4.0 mg, $25.0 \mu \mathrm{mol}, 1.00 \mathrm{eq}$.) and enantiomerically pure 3 $(1.1 \mathrm{mg}, 2.5 \mu \mathrm{mol}, 10 \mathrm{~mol} \%)$ were dissolved in $\alpha, \alpha, \alpha$-trifluorotoluene $(10 \mathrm{~mL}, c=$ $2.5 \mathrm{mmol} / \mathrm{L})$. After degassing the solution, 3-buten-2-one (104 $\mu \mathrm{l}, 1.25 \mathrm{mmol}$, 50.00 eq.) was added and the mixture was irradiated at $419 \mathrm{~nm}$ for $8 \mathrm{~h}$ at $-25^{\circ} \mathrm{C}$. After removing the solvent, purification was achieved by flash column chromatography (silica, pentane/ethyl acetate 2:1). The title compound $4 \mathbf{l}(4.1 \mathrm{mg}, 18.0 \mu \mathrm{mol}, 72 \%$, $95 \%$ ee) was obtained as a colorless solid.

\section{Major regioisomer $(4 \mathrm{ll})$ :}

M.p.: $154-156^{\circ} \mathrm{C}$.

TLC: $R_{\mathrm{f}}=0.66$ (ethyl acetate, UV).

${ }^{1}$ H-NMR (500 MHz, $\left.\mathrm{CDCl}_{3}, 303 \mathrm{~K}\right): \delta(\mathrm{ppm})=9.21$ (br. s, $\left.1 \mathrm{H}, \mathrm{NH}\right), 6.97\left(\mathrm{~d},{ }^{3} \mathrm{~J}=7.6\right.$ $\mathrm{Hz}, 1 \mathrm{H}, \mathrm{H}-8), 6.80\left(\mathrm{dd},{ }^{3} J=7.6 \mathrm{~Hz},{ }^{4} J=2.1 \mathrm{~Hz}, 1 \mathrm{H}, \mathrm{H}-7\right), 6.65\left(\mathrm{~d},{ }^{3} J=2.1 \mathrm{~Hz}, 1 \mathrm{H}, \mathrm{H}-\right.$ 5), 3.91 (virt. t, ${ }^{3} J \approx{ }^{3} J=8.8 \mathrm{~Hz}, 1 \mathrm{H}, \mathrm{H}-8 \mathrm{~b}$ ), 3.43 (virt. $\mathrm{td},{ }^{3} J \approx{ }^{3} J=10.1 \mathrm{~Hz},{ }^{3} J=8.2 \mathrm{~Hz}$, $1 \mathrm{H}, \mathrm{H}-1$ ), 3.23 (virt. td, ${ }^{3} J \approx^{3} J=8.8 \mathrm{~Hz},{ }^{3} J=2.6 \mathrm{~Hz}, 1 \mathrm{H}, \mathrm{H}-2 \mathrm{a}$ ), 2.79 (virt. dt, ${ }^{2} J=11.8$ $\left.\mathrm{Hz},{ }^{3} J \approx{ }^{3} J=9.8 \mathrm{~Hz}, 1 \mathrm{H}, \mathrm{H}-2\right), 2.57\left(\mathrm{ddd},{ }^{2} J=11.8 \mathrm{~Hz},{ }^{3} J=9.2 \mathrm{~Hz},{ }^{3} J=2.6 \mathrm{~Hz}, 1 \mathrm{H}, \mathrm{H}-\right.$ 2), 2.30 (s, 3H, Me), 2.10 (s, 3H, $\left.\mathrm{COCH}_{3}\right)$.

${ }^{13}$ C-NMR $\left(100 \mathrm{MHz}, \mathrm{CDCl}_{3}, 303 \mathrm{~K}\right): \delta(\mathrm{ppm})=207.2\left(\mathrm{~s}, \mathrm{COCH}_{3}\right), 173.2(\mathrm{~s}, \mathrm{C}-3)$, 138.7 (s, C-6), 136.9 (s, C-4a), 122.8 (d, C-8), 124.6 (d, C-7), 120.0 (s, C-8a), 116.5 (d, C-5), 53.0 (d, C-1), 39.1 (d, C-8b), 34.4 (d, C-2a), 28.6 (t, C-2), 28.2 (q, $\mathrm{COCH}_{3}$ ), 21.3 (q, Me). 
IR (ATR): $v\left(\mathrm{~cm}^{-1}\right)=1696,1662,1267,1588,1524,1487,1407,1361,1348,1314$, 1305, 1265, 1204, 1174, 871, 835, 801, 638.

MS (EI, $70 \mathrm{eV}): m / z(\%)=229(6)\left[\mathrm{M}^{+}\right], 186(55)\left[\mathrm{M}^{+}-\mathrm{C}_{2} \mathrm{H}_{3} \mathrm{O}\right], 159$ (100) $\left[\mathrm{M}^{+}-\right.$ $\left.\mathrm{C}_{4} \mathrm{H}_{6} \mathrm{O}\right], 130$ (29), 115 (4), 103 (5), 77 (5).

HRMS (EI) $\left(\mathrm{C}_{14} \mathrm{H}_{15} \mathrm{NO}_{2}\right)$ : calc.: 229.1097 ; found: 229.1097 .

HPLC (AD-H, 250×4.6, $n$-hexane/iso-propanol $=90: 10)=4 \mathbf{l}, t_{\mathrm{R}}=16.4 \mathrm{~min} ;$ ent-4l, $21.2 \mathrm{~min})$.

\section{Minor regioisomer $(\mathbf{S 1 7})$ :}

TLC: $R_{\mathrm{f}}=0.72$ (ethyl acetate, UV).

${ }^{1} \mathrm{H}-\mathrm{NMR}\left(500 \mathrm{MHz}, \mathrm{CDCl}_{3}, 303 \mathrm{~K}\right): \delta(\mathrm{ppm})=7.59$ (br. s, $\left.1 \mathrm{H}, \mathrm{NH}\right), 6.96\left(\mathrm{~d},{ }^{3} J=7.7\right.$ $\mathrm{Hz}, 1 \mathrm{H}, \mathrm{H}-8), 6.83$ (d, $\left.{ }^{3} J=7.7 \mathrm{~Hz}, 1 \mathrm{H}, \mathrm{H}-7\right), 6.50$ (s, 1H, H-5), 3.69 (virt. td, ${ }^{3} J \approx{ }^{3} J=$ $\left.9.0 \mathrm{~Hz},{ }^{3} J=5.5 \mathrm{~Hz}, 1 \mathrm{H}, \mathrm{H}-8 \mathrm{~b}\right), 3.58$ (virt. dt, ${ }^{3} J=9.4 \mathrm{~Hz},{ }^{3} J \approx{ }^{3} J=7.3 \mathrm{~Hz}, 1 \mathrm{H}, \mathrm{H}-2$ ), 3.53 (virt. t, $\left.{ }^{3} J \approx{ }^{3} J=7.4 \mathrm{~Hz}, 1 \mathrm{H}, \mathrm{H}-2 \mathrm{a}\right), 2.89-2.71(\mathrm{~m}, 1 \mathrm{H}, \mathrm{H}-1), 2.31$ (s, 3H, Me), $2.29-2.24(\mathrm{~m}, 1 \mathrm{H}, \mathrm{H}-1), 2.23\left(\mathrm{~s}, 3 \mathrm{H}, \mathrm{COCH}_{3}\right)$.

${ }^{13}$ C-NMR $\left(100 \mathrm{MHz}, \mathrm{CDCl}_{3}, 303 \mathrm{~K}\right): \delta(\mathrm{ppm})=207.1\left(\mathrm{~s}, \mathrm{COCH}_{3}\right), 170.0$ (s, C-3), 138.3 (s, C-6), 135.9 (s, C-4a), 128.6 (d, C-8), 125.0 (d, C-7), 121.5 (s, C-8a), 116.3 (d, C-5), 49.6 (d, C-2), 40.3 (d, C-2a), 32.5 (d, C-8b), 31.9 (t, C-1), 27.9 (q, $\left.\mathrm{COCH}_{3}\right), 21.3$ (q, Me).

MS (EI, $70 \mathrm{eV}): m / z(\%)=229(6)\left[\mathrm{M}^{+}\right], 186(23)\left[\mathrm{M}^{+}-\mathrm{C}_{2} \mathrm{H}_{3} \mathrm{O}\right], 159$ (100) $\left[\mathrm{M}^{+}-\right.$ $\mathrm{C}_{4} \mathrm{H}_{6} \mathrm{O}$ ], 130 (19), 115 (3), 103 (3), 77 (3).

HRMS (EI) $\left(\mathrm{C}_{14} \mathrm{H}_{15} \mathrm{NO}_{2}\right)$ : calc.: 229.1097; found: 229.1096 . 
$(1 R, 2 \mathrm{a} R, 8 \mathrm{bS})$-1-Acetyl-5-methyl-2,2a,4,8b-tetrahydrocyclobuta[c]quinolin-3(1H)one $(4 \mathrm{~m})$
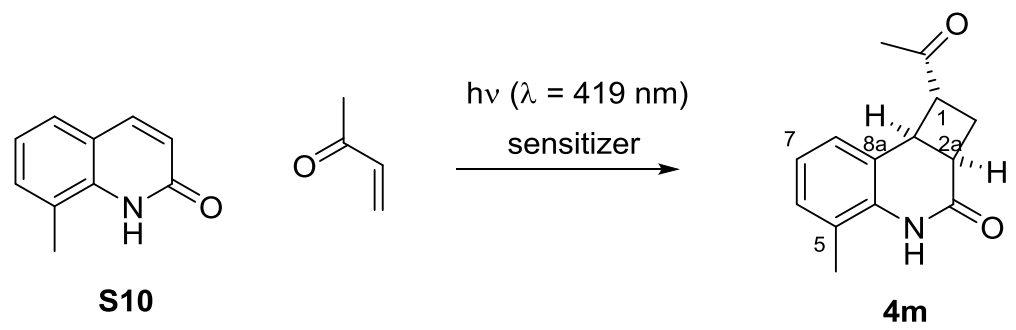

$$
\begin{gathered}
\mathrm{C}_{14} \mathrm{H}_{15} \mathrm{NO}_{2} \\
\text { MW: } 229.28 \mathrm{~g} / \mathrm{mol}
\end{gathered}
$$

Racemic [2+2] Photocycloaddition

8-Methylquinolin-2(1H)-one $(22.0 \mathrm{mg}, 0.14 \mathrm{mmol}, 1.00 \mathrm{eq}$.$) and thioxanthen-9-one$ (15 mg, $0.07 \mathrm{mmol}, 0.50$ eq.) were dissolved in acetonitrile $(10 \mathrm{~mL}, c=14 \mathrm{mmol} / \mathrm{L})$. After degassing the solution, 3-buten-2-one (115 $\mu \mathrm{l}, 1.4 \mathrm{mmol}, 10.00 \mathrm{eq}$.) was added and the mixture was irradiated at $419 \mathrm{~nm}$ for $7 \mathrm{~h}$ at room temperature. After removing the solvent, purification was achieved by flash column chromatography (silica, pentane/ethyl acetate 2:1). The title compound $4 \mathrm{~m}$ (20.6 $\mathrm{mg}, 0.09 \mathrm{mmol}, 65 \%)$ was obtained as a colorless solid. A second fraction was collected containing a mixture of two non-characterized cyclobutanes $(4.4 \mathrm{mg}, 0.02 \mathrm{mmol}, 14 \%, R \mathrm{f}=0.74$ (ethyl acetate, UV)).

\section{Enantioselective [2+2] Photocycloaddition}

8-Methylquinolin-2(1H)-one (4.0 mg, $25.0 \mu \mathrm{mol}, 1.00$ eq.) and enantiomerically pure 3 (1.1 mg, $2.5 \mu \mathrm{mol}, 10 \mathrm{~mol} \%)$ were dissolved in $\alpha, \alpha, \alpha$-trifluorotoluene $(10 \mathrm{~mL}, c=$ $2.5 \mathrm{mmol} / \mathrm{L})$. After degassing the solution, 3-buten-2-one (104 $\mu \mathrm{l}, 1.25 \mathrm{mmol}$, 50.00 eq.) was added and the mixture was irradiated at $419 \mathrm{~nm}$ for $9 \mathrm{~h}$ at $-25^{\circ} \mathrm{C}$. After removing the solvent, purification was achieved by flash column chromatography (silica, pentane/ethyl acetate 2:1). The title compound $\mathbf{4 m}(3.9 \mathrm{mg}, 17 \mu \mathrm{mol}, 68 \%, 80$ $\%$ ee) was obtained as a colorless solid.

M.p.: $146-148{ }^{\circ} \mathrm{C}$.

TLC: $R_{\mathrm{f}}=0.68$ (ethyl acetate, UV).

${ }^{1}$ H-NMR $\left(500 \mathrm{MHz}, \mathrm{CDCl}_{3}, 303 \mathrm{~K}\right): \delta(\mathrm{ppm})=7.70$ (br. s, $\left.1 \mathrm{H}, \mathrm{NH}\right), 7.04\left(\mathrm{~d},{ }^{3} J=7.1\right.$ $\mathrm{Hz}, 1 \mathrm{H}, \mathrm{H}-6), 6.96\left(\mathrm{~d},{ }^{3} J=7.4 \mathrm{~Hz}, 1 \mathrm{H}, \mathrm{H}-8\right), 6.90$ (virt. $\mathrm{t},{ }^{3} J \approx{ }^{3} J=7.3 \mathrm{~Hz}, 1 \mathrm{H}, \mathrm{H}-7$ ), 3.94 (virt. t, ${ }^{3} J \approx{ }^{3} J=8.9 \mathrm{~Hz}, 1 \mathrm{H}, \mathrm{H}-8 \mathrm{~b}$ ), 3.45 (virt. $\mathrm{td},{ }^{3} J \approx{ }^{3} J=9.9 \mathrm{~Hz},{ }^{3} J=7.9 \mathrm{~Hz}, 1 \mathrm{H}$, $\mathrm{H}-1$ ), 3.20 (virt. td, ${ }^{3} J \approx{ }^{3} J=10.0 \mathrm{~Hz},{ }^{3} J=2.8 \mathrm{~Hz}, 1 \mathrm{H}, \mathrm{H}-2 \mathrm{a}$ ), 2.76 (virt. $\mathrm{dt},{ }^{4} J=11.8$ $\left.\mathrm{Hz},{ }^{3} J \approx{ }^{3} J=9.8 \mathrm{~Hz}, 1 \mathrm{H}, \mathrm{H}-2\right), 2.56\left(\mathrm{ddd},{ }^{3} J=11.8 \mathrm{~Hz},{ }^{3} J=9.0 \mathrm{~Hz},{ }^{3} J=2.8 \mathrm{~Hz}, 1 \mathrm{H}, \mathrm{H}-\right.$ 2), 2.25 (s, 3H, Me), 2.09 (s, 3H, $\left.\mathrm{COCH}_{3}\right)$.

${ }^{13}$ C-NMR $\left(100 \mathrm{MHz}, \mathrm{CDCl}_{3}, 303 \mathrm{~K}\right): \delta(\mathrm{ppm})=207.0\left(\mathrm{~s}, \mathrm{COCH}_{3}\right), 172.2(\mathrm{~s}, \mathrm{C}-3)$, 135.1 (s, C-4a), 130.0 (d, C-6), 125.9 (d, C-8), 123.2 (d, C-7), 122.7 (s, C-8a), 122.6 (s, C-5), 52.7 (d, C-1), 39.6 (d, C-8b), 34.3 (d, C-2a), 28.8 (t, C-2), 28.2 (q, $\left.\mathrm{COCH}_{3}\right), 16.9$ (q, Me).

IR (ATR): $v\left(\mathrm{~cm}^{-1}\right)=1715,1698,1666,1617,1602,1507,1489,1457,1447,1417$, 1389, 1362, 1311, 1248, 1233, 1193, 1175, 1151, 954, 842, 818, 796, 736, 729, 703, 620.

MS (EI, $70 \mathrm{eV}): m / z(\%)=229(7)\left[\mathrm{M}^{+}\right], 186(51)\left[\mathrm{M}^{+}-\mathrm{C}_{2} \mathrm{H}_{3} \mathrm{O}\right], 159$ (100) $\left[\mathrm{M}^{+}-\right.$ $\left.\mathrm{C}_{4} \mathrm{H}_{6} \mathrm{O}\right], 141$ (14), 130 (21), 114 (5), 103 (4), 77 (4). 
HRMS (EI) $\left(\mathrm{C}_{14} \mathrm{H}_{15} \mathrm{NO}_{2}\right)$ : calc.: 229.1097 ; found: 229.1098 .

Chiral HPLC (AS-H, 250×4.6, $n$-hexane/iso-propanol = 70:30) $=\mathbf{4 m}, t_{\mathrm{R}}=11.6 \mathrm{~min}$; ent-4m, $14.7 \mathrm{~min})$. 
$(1 R, 2 \mathrm{a} R, 8 \mathrm{~b} S)-3-0 x 0-1,2,2 \mathrm{a}, 3,4,8 \mathrm{~b}-$-hexahydrocyclobuta[c]quinoline-1-carbaldehyde (4n)<smiles>O=c1ccc2ccccc2[nH]1</smiles><smiles>C=CC=O</smiles>

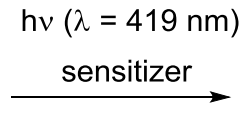<smiles></smiles>

$4 n$

$\mathrm{C}_{12} \mathrm{H}_{11} \mathrm{NO}_{2}$ MW: $201,23 \mathrm{~g} / \mathrm{mol}$

\section{Racemic [2+2] Photocycloaddition}

2(1H)-Quinolone (20 mg, $0.14 \mathrm{mmol}, 1.00$ eq.) and thioxanthen-9-one (15 mg, $0.07 \mathrm{mmol}, 0.50$ eq.) were dissolved in acetonitrile $(10 \mathrm{~mL}, c=14 \mathrm{mmol} / \mathrm{L})$. After degassing the solution, acrolein ( $92 \mu \mathrm{l}, 1.4 \mathrm{mmol}, 10.00$ eq.) was added and the mixture was irradiated at $419 \mathrm{~nm}$ for $8 \mathrm{~h}$ at room temperature. After removing the solvent, purification was achieved by flash column chromatography (silica, pentane/ethyl acetate 1:1). The title compound $4 \mathrm{n}(17.6 \mathrm{mg}, 0.07 \mathrm{mmol}, 47 \%)$ was obtained as a colorless solid.

\section{Enantioselective [2+2] Photocycloaddition}

2(1H)-Quinolone (3.6 mg, $25.0 \mu \mathrm{mol}, 1.00$ eq.) and enantiomerically pure 3 (1.1 mg, $2.5 \mu \mathrm{mol}, 10 \mathrm{~mol} \%$ ) were dissolved in $\alpha, \alpha, \alpha$-trifluorotoluene $(10 \mathrm{~mL}, c=2.5 \mathrm{mmol} / \mathrm{L})$. After degassing the solution, acrolein ( $83 \mu \mathrm{l}, 1.25 \mathrm{mmol}, 50.00$ eq.) was added and the mixture was irradiated at $419 \mathrm{~nm}$ for $18 \mathrm{~h}$ at $-25{ }^{\circ} \mathrm{C}$. After removing the solvent, purification was achieved by flash column chromatography (silica, pentane/ethyl acetate 1:1). The title compound $4 \mathrm{n}(3.0 \mathrm{mg}, 11.0 \mu \mathrm{mol}, 44 \%, 91 \%$ ee $)$ was obtained as a colorless solid.

M.p.: Decomposition.

TLC: $R_{\mathrm{f}}=0.58$ (ethyl acetate, UV).

${ }^{1} \mathbf{H}-N M R\left(500 \mathrm{MHz}, \mathrm{CDCl}_{3}, 303 \mathrm{~K}\right): \delta(\mathrm{ppm})=9.81(\mathrm{~s}, 1 \mathrm{H}, \mathrm{CHO}), 8.89$ (br. s, $1 \mathrm{H}$, $\mathrm{NH}), 7.19$ (virt. td, $\left.{ }^{3} J \approx{ }^{3} J=7.6 \mathrm{~Hz},{ }^{4} J=1.5 \mathrm{~Hz}, 1 \mathrm{H}, \mathrm{H}-6\right), 7.08\left(\mathrm{dd},{ }^{3} J=7.5 \mathrm{~Hz},{ }^{4} J=1.5\right.$ $\mathrm{Hz}, 1 \mathrm{H}, \mathrm{H}-8$ ), 7.00 (virt. td, ${ }^{3} J \approx{ }^{3} J=7.4 \mathrm{~Hz},{ }^{4} J=1.2 \mathrm{~Hz}, 1 \mathrm{H}, \mathrm{H}-7$ ), 6.81 (dd, ${ }^{3} J=8.0$ $\mathrm{Hz},{ }^{4} J=1.2 \mathrm{~Hz}, 1 \mathrm{H}, \mathrm{H}-5$ ), 4.08 (virt. $\mathrm{t},{ }^{3} J \approx{ }^{3} J=8.7 \mathrm{~Hz}, 1 \mathrm{H}, \mathrm{H}-8 \mathrm{~b}$ ), 3.45 (virt. $\mathrm{td},{ }^{3} J \approx^{3} J$ $=9.2 \mathrm{~Hz},{ }^{3} J=8.0 \mathrm{~Hz}, 1 \mathrm{H}, \mathrm{H}-1$ ), 3.31 (virt. td, ${ }^{3} J \approx{ }^{3} J=9.3 \mathrm{~Hz},{ }^{3} J=3.4 \mathrm{~Hz}, 1 \mathrm{H}, \mathrm{H}-2 \mathrm{a}$ ), 2.84 (virt. dt, $\left.{ }^{2} J=12.0 \mathrm{~Hz},{ }^{3} J \approx{ }^{3} J=9.8 \mathrm{~Hz}, 1 \mathrm{H}, \mathrm{H}-2\right), 2.62\left(\mathrm{ddd},{ }^{2} J=12.0 \mathrm{~Hz},{ }^{3} J=9.3\right.$ $\left.\mathrm{Hz},{ }^{3} \mathrm{~J}=3.4 \mathrm{~Hz}, 1 \mathrm{H}, \mathrm{H}-2\right)$.

${ }^{13}$ C-NMR $\left(100 \mathrm{MHz}, \mathrm{CDCl}_{3}, 303 \mathrm{~K}\right): \delta(\mathrm{ppm})=200.1(\mathrm{~s}, \mathrm{CHO}), 172.1$ (s, C-3), 136.8 (s, C-4a), 128.6 (d, C-6), 128.1 (d, C-8), 124.0 (d, C-7), 122.4 (s, C-8a), 116.0 (d, C-5), 52.4 (d, C-1), 37.4 (d, C-8b), 34.8 (d, C-2a), 27.0 (t, C-2).

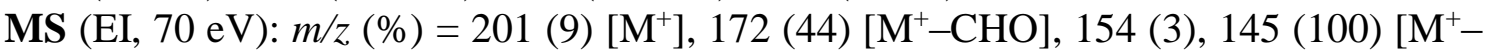
$\left.\mathrm{C}_{3} \mathrm{H}_{4} \mathrm{O}\right], 117$ (42), 90 (14), 77 (3).

HRMS (EI) $\left(\mathrm{C}_{12} \mathrm{H}_{11} \mathrm{NO}_{2}\right)$ : calc.: 201.0784; found: 201.0789 .

HPLC $(\mathrm{OJ}-\mathrm{H}, 250 \times 4.6, n$-hexane/iso-propanol $=90: 10)=e n t-4 \mathbf{n}, t_{\mathrm{R}}=19.4 \mathrm{~min} ; \mathbf{4 n}$, $22.6 \mathrm{~min})$. 

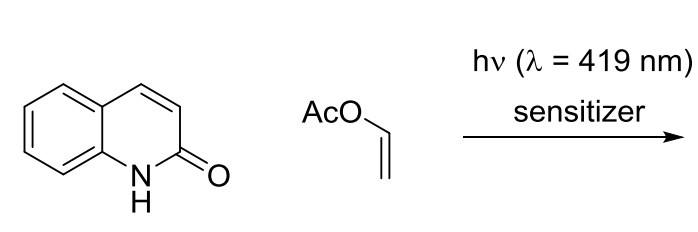
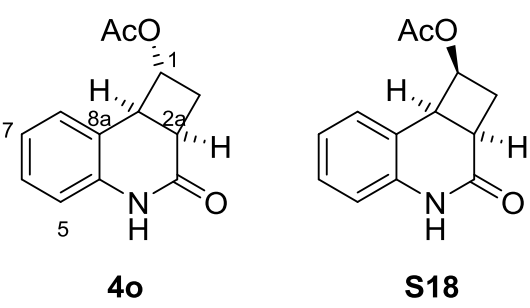

$\mathrm{C}_{13} \mathrm{H}_{13} \mathrm{NO}_{3}$ MW: $231,25 \mathrm{~g} / \mathrm{mol}$

\section{Racemic [2+2] Photocycloaddition}

2(1H)-Quinolone (20 mg, $0.14 \mathrm{mmol}, 1.00$ eq.) and thioxanthen-9-one (15 mg, $0.07 \mathrm{mmol}, 0.50$ eq.) were dissolved in acetonitrile $(10 \mathrm{~mL}, c=14 \mathrm{mmol} / \mathrm{L})$. After degassing the solution, vinyl acetate (127 $\mu \mathrm{l}, 1.4 \mathrm{mmol}, 10.00$ eq.) was added and the mixture was irradiated at $419 \mathrm{~nm}$ for $3 \mathrm{~h}$ at room temperature. After removing the solvent, purification was achieved by flash column chromatography (silica, pentane/ethyl acetate 6:4). The title compounds $\mathbf{4 o}(14.8 \mathrm{mg}, 64.0 \mu \mathrm{mol}, 46 \%)$ and $\mathbf{S 1 8}$ (13.2 mg, $57.8 \mu \mathrm{mol}, 40 \%$ ) were obtained as colorless solids.

\section{Enantioselective [2+2] Photocycloaddition}

2(1H)-Quinolone (3.6 mg, $25.0 \mu \mathrm{mol}, 1.00 \mathrm{eq}$.) and enantiomerically pure $3(1.1 \mathrm{mg}$, $2.5 \mu \mathrm{mol}, 10 \mathrm{~mol} \%)$ were dissolved in $\alpha, \alpha, \alpha$-trifluorotoluene $(10 \mathrm{~mL}, c=2.5 \mathrm{mmol} / \mathrm{L})$. After degassing the solution, vinyl acetate (115 $\mu \mathrm{l}, 1.25 \mathrm{mmol}, 50.00$ eq.) was added and the mixture was irradiated at $419 \mathrm{~nm}$ for $9 \mathrm{~h}$ at $-25{ }^{\circ} \mathrm{C}(87 \%$ conversion $)$. After removing the solvent, purification was achieved by flash column chromatography (silica, pentane/ethyl acetate 6:4). The title compounds 40 (2.4 mg, $10.3 \mu \mathrm{mol}, 42 \%$, $58 \% e e, 48 \%$ based on recovered starting material) and S18 (2.1 mg, $9.08 \mu \mathrm{mol}, 41 \%$, $43 \%$ ee) were obtained as a colorless solid.

Exo isomer (4o):

M.p.: $181^{\circ} \mathrm{C}$.

TLC: $R_{\mathrm{f}}=0.31$ (cylohexane:ethyl acetate, $1: 1, \mathrm{UV}$ ).

${ }^{1}$ H-NMR (500 MHz, $\left.\mathrm{CDCl}_{3}, 303 \mathrm{~K}\right): \delta(\mathrm{ppm})=7.99$ (br. s, $\left.1 \mathrm{H}, \mathrm{NH}\right), 7.22-7.12$ (m, $2 \mathrm{H}, \mathrm{H}-6, \mathrm{H}-8$ ), 7.00 (virt. td, ${ }^{3} J \approx{ }^{3} J=7.5 \mathrm{~Hz},{ }^{4} J=1.2 \mathrm{~Hz}, 1 \mathrm{H}, \mathrm{H}-7$ ), $6.72\left(\mathrm{dd},{ }^{3} J=7.9\right.$ $\left.\mathrm{Hz},{ }^{4} J=1.2 \mathrm{~Hz}, 1 \mathrm{H}, \mathrm{H}-5\right), 5.12\left(\mathrm{dt},{ }^{3} J=7.6 \mathrm{~Hz},{ }^{3} J \approx{ }^{3} J=6.2 \mathrm{~Hz}, 1 \mathrm{H}, \mathrm{H}-1\right), 3.78\left(\mathrm{dd},{ }^{3} J\right.$ $\left.=10.0 \mathrm{~Hz},{ }^{3} J=6.5 \mathrm{~Hz}, 1 \mathrm{H}, \mathrm{H}-8 \mathrm{~b}\right), 3.36\left(\mathrm{dddd},{ }^{3} J=11.1 \mathrm{~Hz},{ }^{3} J=9.9 \mathrm{~Hz},{ }^{3} J=3.6 \mathrm{~Hz},{ }^{4} J\right.$ $=1.3 \mathrm{~Hz}, 1 \mathrm{H}, \mathrm{H}-2 \mathrm{a}), 2.89\left(\mathrm{dddd},{ }^{2} J=12.3 \mathrm{~Hz},{ }^{3} J=7.5 \mathrm{~Hz},{ }^{3} J=3.6 \mathrm{~Hz},{ }^{4} J=1.1 \mathrm{~Hz}, 1 \mathrm{H}\right.$, $\mathrm{H}-2), 2.70-2.60(\mathrm{~m}, 1 \mathrm{H}, \mathrm{H}-2), 2.08\left(\mathrm{~s}, 3 \mathrm{H}, \mathrm{CH}_{3}\right)$. 
${ }^{13}$ C-NMR $\left(100 \mathrm{MHz}, \mathrm{CDCl}_{3}, 303 \mathrm{~K}\right): \delta(\mathrm{ppm})=171.5(\mathrm{~s}, \mathrm{C}-3), 170.2\left(\mathrm{~s}, \mathrm{OCOCH}_{3}\right)$, 136.9 (s, C-4a), 128.7 (d, C-6), 128.5 (d, C-8), 123.8 (d, C-7), 121.2 (s, C-8a), 115.6 (d, C-5), 74.0 (d, C-1), 44.9 (d, C-8b), 35.2 (t, C-2), 31.7 (d, C-2a), 21.0 (q, $\mathrm{CH}_{3}$ ).

IR (ATR): $v\left(\mathrm{~cm}^{-1}\right)=3202,3063,1738,1670,1594,1491,1436,1378,1327,1303$, 1236, 1185, 1082, 1051, 815, 755, 733 .

MS (EI, $70 \mathrm{eV}): m / z(\%)=231(5)\left[\mathrm{M}^{+}\right], 188(14)\left[\mathrm{M}^{+}-\mathrm{C}_{2} \mathrm{H}_{3} \mathrm{O}\right], 145$ (100) $\left[\mathrm{M}^{+}-\right.$ $\left.\mathrm{C}_{4} \mathrm{H}_{6} \mathrm{O}_{2}\right], 117$ (22).

HRMS (EI) $\left(\mathrm{C}_{13} \mathrm{H}_{13} \mathrm{NO}_{3}\right)$ : calc.: 231.0890; found: 231.0892 .

HPLC $($ AD-H, 250×4.6, $n$-hexane/iso-propanol $=90: 10)=4 \mathbf{o}, t_{\mathrm{R}}=16.2 \mathrm{~min}$; ent-4o, $30.7 \mathrm{~min})$.

Endo isomer (S18):

M.p.: $153^{\circ} \mathrm{C}$.

TLC: $R \mathrm{f}=0.24$ (cylohexane:ethyl acetate, $1: 1, \mathrm{UV}$ ).

${ }^{1} \mathbf{H}-\mathbf{N M R}\left(500 \mathrm{MHz}, \mathrm{CDCl}_{3}, 303 \mathrm{~K}\right): \delta(\mathrm{ppm})=8.44$ (br. s, $\left.1 \mathrm{H}, \mathrm{NH}\right), 7.19$ (dddd, ${ }^{3} J=$ $7.8 \mathrm{~Hz},{ }^{3} J=7.0 \mathrm{~Hz},{ }^{4} J=1.8 \mathrm{~Hz},{ }^{4} J=0.7 \mathrm{~Hz}, 1 \mathrm{H}, \mathrm{H}-6$ ), 6.99 (virt. td, ${ }^{3} J \approx{ }^{3} J=7.4 \mathrm{~Hz},{ }^{4} J$ $=1.2 \mathrm{~Hz}, 1 \mathrm{H}, \mathrm{H}-7), 6.95\left(\mathrm{ddd},{ }^{3} J=7.7 \mathrm{~Hz},{ }^{4} J=1.8 \mathrm{~Hz},{ }^{4} J=0.8 \mathrm{~Hz}, \mathrm{H}-8\right), 6.78\left(\mathrm{dd},{ }^{3} J=\right.$ $7.9 \mathrm{~Hz},{ }^{4} J=1.2 \mathrm{~Hz}, 1 \mathrm{H}, \mathrm{H}-5$ ), 5.42 (virt. qd, ${ }^{3} J \approx{ }^{3} J \approx{ }^{3} J=7.3 \mathrm{~Hz},{ }^{4} J=0.6 \mathrm{~Hz}, 1 \mathrm{H}, \mathrm{H}-1$ ), $4.19-4.12$ (m, $1 \mathrm{H}, \mathrm{H}-8 \mathrm{~b}$ ), 3.21 (virt. q, ${ }^{3} J \approx{ }^{3} J \approx{ }^{3} J=8.6 \mathrm{~Hz}, 1 \mathrm{H}, \mathrm{H}-2 \mathrm{a}$ ), 2.95 (dddd, ${ }^{2} J$ $\left.=12.2 \mathrm{~Hz},{ }^{3} \mathrm{~J}=8.9 \mathrm{~Hz},{ }^{3} \mathrm{~J}=7.3 \mathrm{~Hz},{ }^{4} \mathrm{~J}=3.1 \mathrm{~Hz}, 1 \mathrm{H}, \mathrm{H}-2\right), 2.53\left(\mathrm{dddd},{ }^{2} J=12.2 \mathrm{~Hz},{ }^{3} J\right.$ $\left.=8.4 \mathrm{~Hz},{ }^{3} \mathrm{~J}=7.3 \mathrm{~Hz},{ }^{4} \mathrm{~J}=1.1 \mathrm{~Hz}, 1 \mathrm{H}, \mathrm{H}-2\right), 1.88\left(\mathrm{~s}, 3 \mathrm{H}, \mathrm{CH}_{3}\right)$.

${ }^{13}$ C-NMR $\left(100 \mathrm{MHz}, \mathrm{CDCl}_{3}, 303 \mathrm{~K}\right): \delta(\mathrm{ppm})=170.45(\mathrm{~s}, \mathrm{C}-3), 170.2\left(\mathrm{~s}, \mathrm{OCOCH}_{3}\right)$, 137.4 (s, C-4a), 130.5 (d, C-8), 128.4 (d, C-6), 123.3 (d, C-7), 117.5 (s, C-8a), 116.0 (d, C-5), 69.4 (d, C-1), 42.5 (d, C-8b), 34.6 (t, C-2), 31.9 (d, C-2a), 20.9 (q, $\mathrm{CH}_{3}$ ).

IR (ATR): $v\left(\mathrm{~cm}^{-1}\right)=3202,3064,2987,1735,1671,1593,1559,1507,1490,1436$, 1388, 1324, 1296, 1235, 1087, 1014, 756, 735.

MS (EI, $70 \mathrm{eV}): m / z(\%)=231(5)\left[\mathrm{M}^{+}\right], 188(14)\left[\mathrm{M}^{+}-\mathrm{C}_{2} \mathrm{H}_{3} \mathrm{O}\right], 145$ (100) $\left[\mathrm{M}^{+}-\right.$ $\left.\mathrm{C}_{4} \mathrm{H}_{6} \mathrm{O}_{2}\right], 117$ (22).

HRMS (EI) $\left(\mathrm{C}_{13} \mathrm{H}_{13} \mathrm{NO}_{3}\right)$ : calc.: 231.0890; found: 231.0893 .

HPLC $\left(A D-H, 250 \times 4.6, n\right.$-hexane/iso-propanol = 95:5) $=\mathbf{S 1 7}, t_{\mathrm{R}}=34.5 \mathrm{~min} ;$ ent-S17, $36.8 \mathrm{~min})$. 

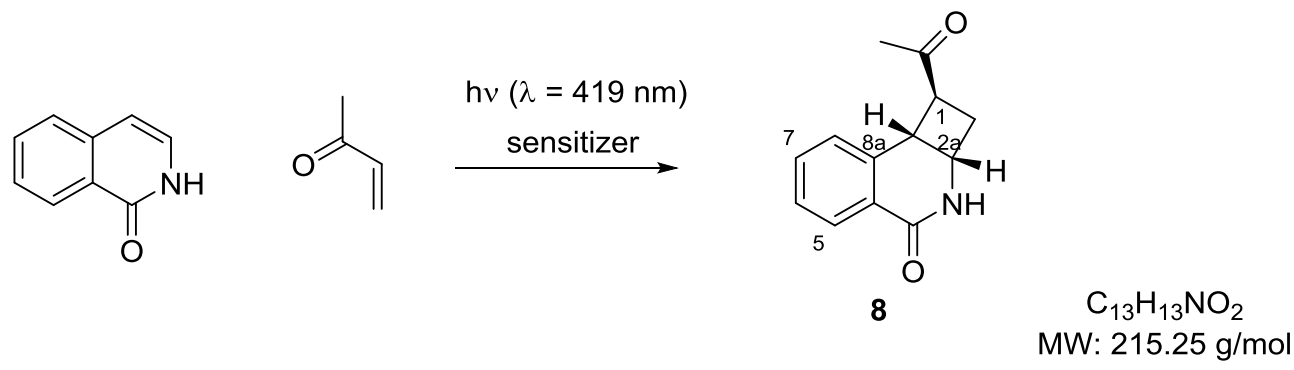

\section{Racemic [2+2] Photocycloaddition}

Isoquinolone (20.0 mg, $0.14 \mathrm{mmol}, 1.00$ eq.) and thioxanthen-9-one (15 mg, $0.07 \mathrm{mmol}$, 0.50 eq.) were dissolved in acetonitrile $(10 \mathrm{~mL}, c=14 \mathrm{mmol} / \mathrm{L})$. After degassing the solution, 3-buten-2-one ( $115 \mu \mathrm{l}, 1.4 \mathrm{mmol}, 10.00 \mathrm{eq}$.) was added and the mixture was irradiated at $419 \mathrm{~nm}$ for $9 \mathrm{~h}$ at room temperature (conversion $93 \%$ ). After removing the solvent, purification was achieved by flash column chromatography (silica, pentane/ethyl acetate 1:1). The title compound 8 (16.6 $\mathrm{mg}, 0.08 \mathrm{mmol}, 56 \%)$ was obtained as a colorless solid.

\section{Enantioselective [2+2] Photocycloaddition}

Isoquinolone (3.6 mg, $25.0 \mu \mathrm{mol}, 1.00$ eq.) and enantiomerically pure $3(1.1 \mathrm{mg}$, $2.5 \mu \mathrm{mol}, 10 \mathrm{~mol} \%$ ) were dissolved in $\alpha, \alpha, \alpha$-trifluorotoluene $(10 \mathrm{~mL}, c=2.5 \mathrm{mmol} / \mathrm{L})$. After degassing the solution, 3-buten-2-one (104 $\mu \mathrm{l}, 1.25 \mathrm{mmol}, 50.00$ eq.) was added and the mixture was irradiated at $419 \mathrm{~nm}$ for $9 \mathrm{~h}$ at $-25{ }^{\circ} \mathrm{C}$ ( $86 \%$ conversion). After removing the solvent, purification was achieved by flash column chromatography (silica, pentane/ethyl acetate 1:1). The title compound 8 (4.0 mg, $19 \mu \mathrm{mol}, 74 \%, 91 \%$ $e e, 86 \%$ based on recovered starting material) was obtained as a colorless solid.

M.p.: $143-145^{\circ} \mathrm{C}$.

TLC: $R \mathrm{f}=0.44$ (ethyl acetate, UV).

${ }^{1} \mathbf{H}$-NMR $\left(500 \mathrm{MHz}, \mathrm{CDCl}_{3}, 303 \mathrm{~K}\right): \delta(\mathrm{ppm})=8.15\left(\mathrm{dd},{ }^{3} \mathrm{~J}=7.9 \mathrm{~Hz},{ }^{4} \mathrm{~J}=1.4 \mathrm{~Hz}, 1 \mathrm{H}\right.$, $\mathrm{H}-5$ ), 7.47 (virt. td, ${ }^{3} J \approx{ }^{3} J=7.5 \mathrm{~Hz},{ }^{4} J=1.4 \mathrm{~Hz}, 1 \mathrm{H}, \mathrm{H}-7$ ), 7.36 (virt. td, ${ }^{3} J \approx{ }^{3} J=7.6$ $\mathrm{Hz},{ }^{4} J=1.2 \mathrm{~Hz}, 1 \mathrm{H}, \mathrm{H}-6$ ), 7.18 (dd, $\left.{ }^{3} J=7.6 \mathrm{~Hz},{ }^{4} J=1.2 \mathrm{~Hz}, 1 \mathrm{H}, \mathrm{H}-8\right), 6.77$ (br. s, $1 \mathrm{H}$, $\mathrm{NH}), 4.32-4.20(\mathrm{~m}, 1 \mathrm{H}, \mathrm{H}-2 \mathrm{a}), 3.99$ (virt. $\left.\mathrm{t},{ }^{3} \mathrm{~J} \approx{ }^{3} \mathrm{~J}=7.7 \mathrm{~Hz}, 1 \mathrm{H}, \mathrm{H}-8 \mathrm{~b}\right), 3.52$ (virt. td, ${ }^{3} J \approx{ }^{3} J=9.0 \mathrm{~Hz},{ }^{3} J=7.5 \mathrm{~Hz}, 1 \mathrm{H}, \mathrm{H}-1$ ), 2.54 (virt. dt, ${ }^{2} J=11.8 \mathrm{~Hz},{ }^{3} J \approx{ }^{3} J=7.2 \mathrm{~Hz}$, $1 \mathrm{H}, \mathrm{H}-2), 2.37\left(\mathrm{ddd},{ }^{2} J=11.8 \mathrm{~Hz},{ }^{3} J=9.3 \mathrm{~Hz},{ }^{3} J=2.4 \mathrm{~Hz}, 1 \mathrm{H}, \mathrm{H}-2\right), 2.14(\mathrm{~s}, 3 \mathrm{H}$, $\left.\mathrm{COCH}_{3}\right)$.

${ }^{13}$ C-NMR $\left(100 \mathrm{MHz}, \mathrm{CDCl}_{3}, 303 \mathrm{~K}\right): \delta(\mathrm{ppm})=207.5\left(\mathrm{~s}, \mathrm{COCH}_{3}\right), 164.5$ (s, C-4), 138.8 (s, C-8a), 133.1 (d, C-7), 128.5 (d, C-5), 127.6 (d, C-6), 127.3 (d, C-8), 126.5 (s, C-4a), 53.3 (d, C-1), 46.5 (d, C-2a), 37.1 (d, C-8b), 33.6 (t, C-2), 28.2 (q, $\mathrm{COCH}_{3}$ ).

IR (ATR): $v\left(\mathrm{~cm}^{-1}\right)=3186,2923,1686,1492,1458,1413,1362,1342,1174,1161$, 811, 786, 766, 734.

MS (EI, $70 \mathrm{eV}): m / z(\%)=215(1)\left[\mathrm{M}^{+}\right], 145(100)\left[\mathrm{M}^{+}-\mathrm{C}_{4} \mathrm{H}_{6} \mathrm{O}\right], 118$ (24), 90 (8).

HRMS (EI) $\left(\mathrm{C}_{13} \mathrm{H}_{13} \mathrm{NO}_{2}\right)$ : calc.: 215.0946; found: 215.0949

HPLC $\left(A D-H, 250 \times 4.6, n\right.$-hexane/iso-propanol = 90:10) $=$ ent-8, $t_{\mathrm{R}}=18.9 \mathrm{~min} ; \mathbf{8}, 29.4$ $\min )$. 


\section{Proof of Constitution and Absolute Configuration}

\section{Regioselectivity and Relative Configuration}

The complete set of ${ }^{1} \mathrm{H}$ and ${ }^{13} \mathrm{C}$ NMR signals was assigned by a combination of $1 \mathrm{D}$ and 2D NMR experiments. HMBC was used to determine the constitution of the isomers while NOESY spectra gave information about the spatial correlations between proximate protons (i.e. relative configuration). The most important HMBC and NOESY correlations for compound $\mathbf{4 a}$ and $\mathbf{S 1 1}$ are depicted in Figure S1 and Figure S2.

Important HMBC correlations (4a):

Important NOESY correlations (4a):

- $\mathrm{C}-3 \rightarrow \mathrm{H}-2, \mathrm{H}-8 \mathrm{~b}$

- $\mathrm{C}-4 \mathrm{a} \rightarrow \mathrm{H}-6, \mathrm{H}-8, \mathrm{H}-8 \mathrm{~b}$

- $\mathrm{C}-8 \rightarrow \mathrm{H}-6, \mathrm{H}-8 \mathrm{~b}$

- $\mathrm{C}-8 \mathrm{a} \rightarrow \mathrm{H}-1, \mathrm{H}-2 \mathrm{a}, \mathrm{N}-\mathrm{H}$

- $\mathrm{N}-\mathrm{H} \leftrightarrow \mathrm{H}-1$

- $\mathrm{N}-\mathrm{H} \leftrightarrow \mathrm{H}-2(2.60 \mathrm{ppm})$

- $\mathrm{N}-\mathrm{H} \leftrightarrow \mathrm{H}-5$

- $\mathrm{H}-8 \mathrm{~b} \leftrightarrow \mathrm{H}-2(2.87 \mathrm{ppm})$
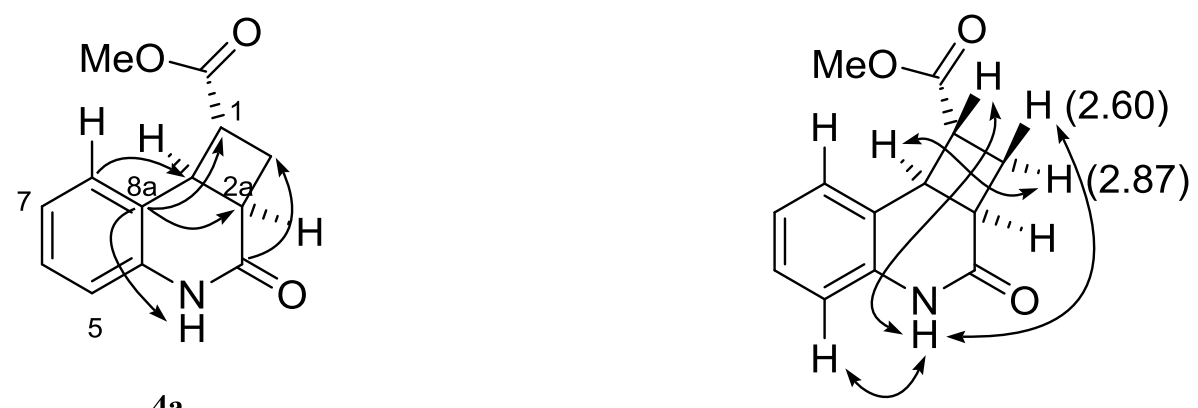

4a

Figure S1: HMBC/NOESY correlations of compound 4a (exo).

Important HMBC correlations (S11):

Important NOESY correlations (S11):

- $\mathrm{C}-3 \rightarrow \mathrm{H}-2, \mathrm{H}-8 \mathrm{~b}$

- $\mathrm{C}-4 \mathrm{a} \rightarrow \mathrm{H}-6, \mathrm{H}-8, \mathrm{H}-8 \mathrm{~b}$

- $\mathrm{N}-\mathrm{H} \leftrightarrow \mathrm{H}-2$ (2.89 ppm)

- $\mathrm{C}-8 \rightarrow \mathrm{H}-6, \mathrm{H}-8 \mathrm{~b}$

- $\mathrm{N}-\mathrm{H} \leftrightarrow \mathrm{H}-5$

- $\mathrm{C}-8 \mathrm{a} \rightarrow \mathrm{H}-1, \mathrm{H}-2 \mathrm{a}, \mathrm{N}-\mathrm{H}$

- $\mathrm{H}-8 \mathrm{~b} \leftrightarrow \mathrm{H}-2$ (2.72 ppm)
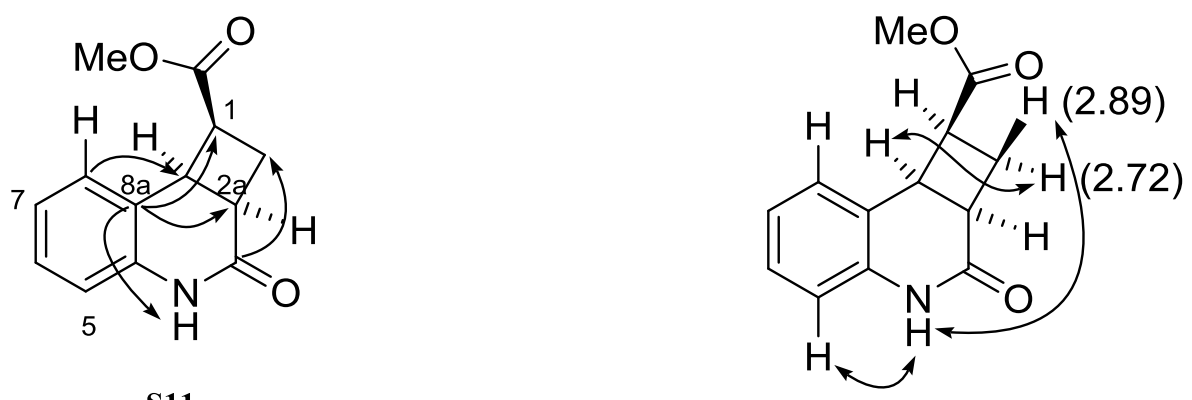

S11

Figure S2: HMBC/NOESY correlations of compound S11 (endo). 
The most important HMBC and NOESY correlations for compound 4d and S14 are depicted in Figure S3 and Figure S4.

Important HMBC correlations (4d): $\quad$ Important NOESY correlations $(\mathbf{4 d})$ :

- $\mathrm{C}-3 \rightarrow \mathrm{H}-2, \mathrm{H}-8 \mathrm{~b}$

- $\mathrm{C}-4 \mathrm{a} \rightarrow \mathrm{H}-6, \mathrm{H}-8, \mathrm{H}-8 \mathrm{~b}$

- $\mathrm{C}-8 \rightarrow \mathrm{H}-6, \mathrm{H}-8 \mathrm{~b}$

- $\mathrm{C}-8 \mathrm{a} \rightarrow \mathrm{H}-2 \mathrm{a}, \mathrm{N}-\mathrm{H}$

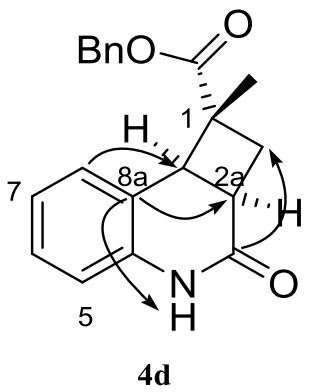

- $\mathrm{N}-\mathrm{H} \leftrightarrow \mathrm{H}-2(2.33 \mathrm{ppm})$

- $\mathrm{N}-\mathrm{H} \leftrightarrow \mathrm{CH}_{3}, \mathrm{H}-5$

- $\mathrm{CH}_{3} \leftrightarrow \mathrm{H}-2(2.33 \mathrm{ppm})$

- $\mathrm{H}-8 \mathrm{~b} \leftrightarrow \mathrm{H}-2(3.21 \mathrm{ppm})$

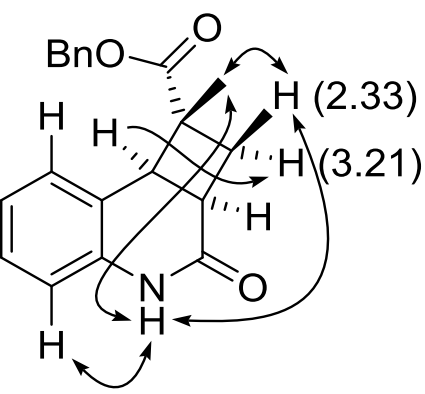

Figure S3: HMBC/NOESY correlations of compound 4d (exo).

Important HMBC correlations (S14):

Important NOESY correlations (S14):

- $\mathrm{C}-3 \rightarrow \mathrm{H}-2, \mathrm{H}-8 \mathrm{~b}$

- $\mathrm{C}-4 \mathrm{a} \rightarrow \mathrm{H}-6, \mathrm{H}-8, \mathrm{H}-8 \mathrm{~b}$

- $\mathrm{C}-8 \rightarrow \mathrm{H}-6, \mathrm{H}-8 \mathrm{~b}$

- $\mathrm{C}-8 \mathrm{a} \rightarrow \mathrm{H}-2 \mathrm{a}, \mathrm{N}-\mathrm{H}$

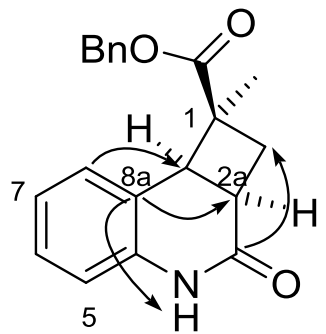

S14
- $\mathrm{N}-\mathrm{H} \leftrightarrow \mathrm{H}-2$ (3.17 ppm), H-5

- $\mathrm{H}-2 \mathrm{a} \leftrightarrow \mathrm{CH}_{3}$

- $\mathrm{H}-8 \mathrm{~b} \leftrightarrow \mathrm{H}-2$ (2.44 ppm), $\mathrm{CH}_{3}$

- $\mathrm{CH}_{3} \leftrightarrow \mathrm{H}-2(2.44 \mathrm{ppm})$

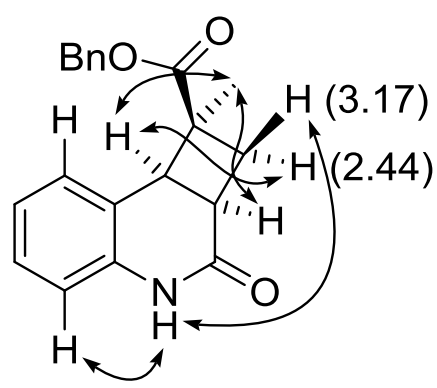

Figure S4: HMBC/NOESY correlations of compound S14 (endo). 


\section{Absolute Configuration}

In previous work [Nature 2005, 436, 1139-1140], it has been established that the dihedral angle of the dihydroquinolone chromophore is responsible for the specific rotation of a given dihydroquinolone. When applied to products of a [2+2] photocycloaddition the relevant dihedral angle relates to atoms $\mathrm{C} 3-\mathrm{N} 4-\mathrm{C} 4 \mathrm{a}-\mathrm{C} 8 \mathrm{a}$, which are in the precursor quinolones atoms $\mathrm{C} 2-\mathrm{N} 1-\mathrm{C} 8 \mathrm{a}-\mathrm{C} 4 \mathrm{a}$. In products with core $\mathbf{A}$, the twist of the chromophore results from the annelated cyclobutane. The dihedral angle becomes negative if the cyclobutane ring and hydrogen atoms at C-2a and C-8b are positioned as shown for $\mathbf{A}$ [in $\mathbf{A}$ this would correlate to an $(R)$-configuration at $\mathrm{C}-2 \mathrm{a}$ ]. Photocycloaddition products with this absolute configuration are levorotatory. This scenario is shown in the adjacent molecular model of $\mathbf{A}$. In the opposite scenario, the dihedral angle is positive and the photocycloaddition products are dextrorotatory.
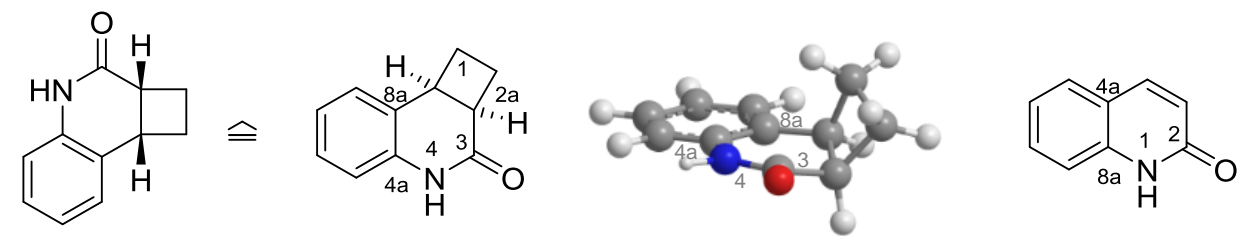

A

The method has been applied to assign the absolute configuration in intramolecular [2+2] photocycloaddition reactions [J. Am. Chem. Soc. 2011, 133, 16689-16697].

To substantiate further the direction of the enantioface differentiation, the reaction of $1(2 H)$-quinolone with ethyl vinyl ketone was performed under conditions of direct excitation $\left(\lambda=366 \mathrm{~nm},-70{ }^{\circ} \mathrm{C}, \mathrm{PhCH}_{3}\right)$ in the presence of stoichiometric amounts (2.5 eq.) of chiral template (+)-T, which has proven in the last 15 years to provide a complete enantioface differentiation according to the model shown below [Review: Bauer, A.; Alonso, R. Templated Enantioselective Photocatalysis. In Chemical Photocatalysis; König, B., Ed.; DeGruyter: Berlin, 2013; pp 67-90]. The product enantiomer $4 \mathbf{f}$ was according to its HPLC trace identical to the product enantiomer obtained with catalyst 3 .
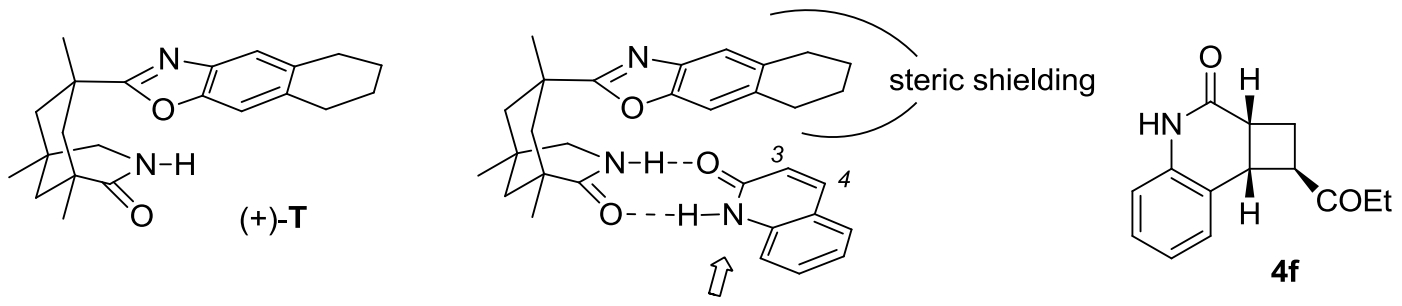
HPLC traces (for details, see section 10)

HPLC trace of $r a c-\mathbf{4 f}$

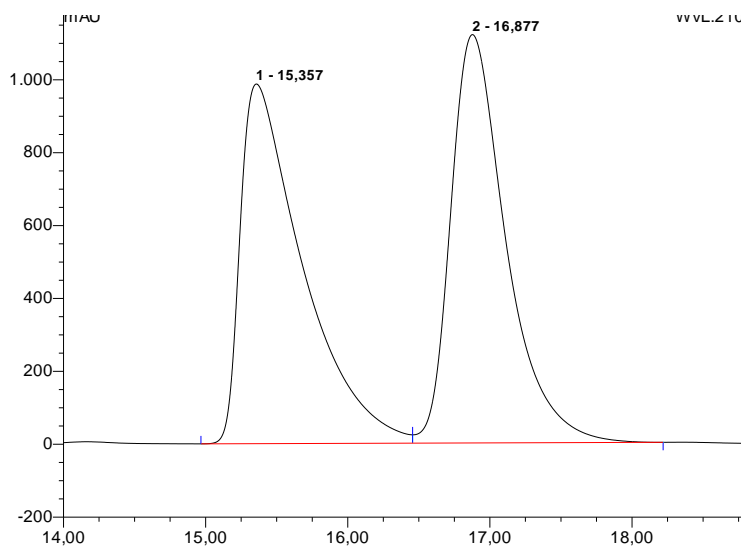

HPLC trace of $\mathbf{4 f}($ from (+)-T)

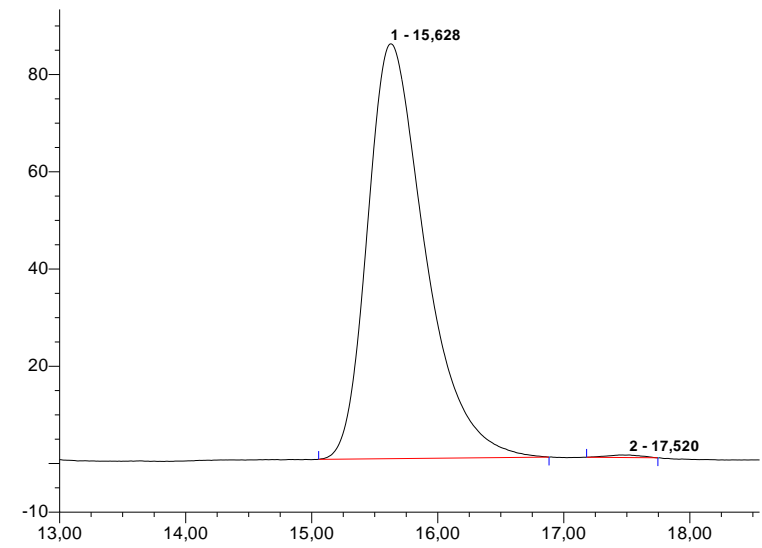

HPLC trace of $\mathbf{4 f}$ (from $\mathbf{3}$ )

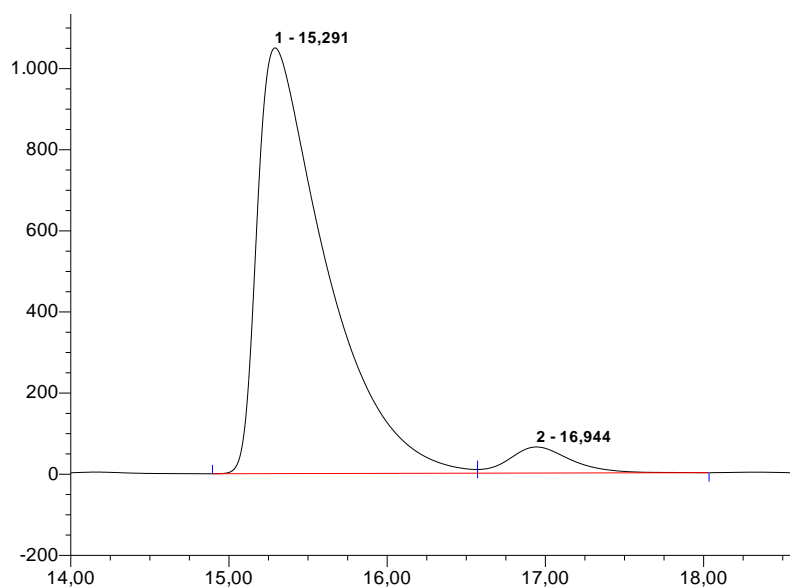




\section{Synthesis of the Thioxanthone Derivative 7}

\section{2-Nitro-9-oxo-9H-thioxanthen-3-yl pivaloate (S19)}<smiles>CCN(CC)C(C)C(C)C</smiles>

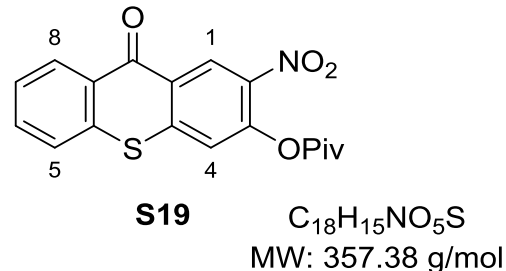

PivCl (356 $\mu \mathrm{L}, 2.90 \mathrm{mmol}, 1.05$ eq.) was added dropwise via syringe to a solution of 3-

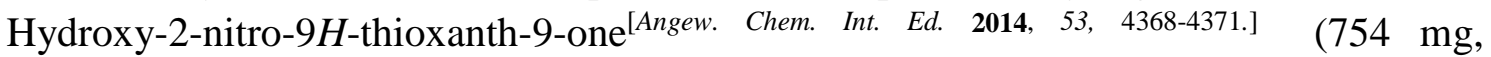
$2.76 \mathrm{mmol}, 1.0$ eq.), $\mathrm{Et}_{3} \mathrm{~N}(1.2 \mathrm{~mL}, 8.3 \mathrm{mmol}, 1.15$ eq.) and $\mathrm{THF}(50 \mathrm{~mL}, c=$ $50 \mathrm{mmol} / \mathrm{L}$ ). The mixture was stirred at room temperature for $30 \mathrm{~min}$ and then poured into $0.1 \mathrm{M} \mathrm{HCl}(200 \mathrm{~mL})$. The precipitate was filtered and dried in the desiccator to yield the desired pivaloate $\mathbf{S 1 9}$ (980 mg, $2.74 \mathrm{mmol}, 99 \%$ ).

M.p.: $233{ }^{\circ} \mathrm{C}$.

TLC: $R \mathrm{f}=0.66$ (cylohexane:ethyl acetate, $1: 1, \mathrm{UV}$ ).

${ }^{1} \mathbf{H}-\mathbf{N M R}\left(500 \mathrm{MHz}, \mathrm{CDCl}_{3}, 303 \mathrm{~K}\right): \delta(\mathrm{ppm})=9.30(\mathrm{~s}, 1 \mathrm{H}, \mathrm{C}-1-\mathrm{H}), 8.61\left(\mathrm{dd},{ }^{3} J=8.1\right.$ $\left.\mathrm{Hz},{ }^{4} \mathrm{~J}=1.6 \mathrm{~Hz}, 1 \mathrm{H}, \mathrm{C}-8-\mathrm{H}\right), 7.70\left(\mathrm{ddd},{ }^{3} \mathrm{~J}=8.4,7.1 \mathrm{~Hz},{ }^{4} \mathrm{~J}=1.5 \mathrm{~Hz}, 1 \mathrm{H}, \mathrm{C}-6-\mathrm{H}\right), 7.61-$ $7.55(\mathrm{~m}, 2 \mathrm{H}, \mathrm{C}-5-\mathrm{H}, \mathrm{C}-7-\mathrm{H}), 7.40(\mathrm{~s}, 1 \mathrm{H}, \mathrm{C}-4-\mathrm{H}), 1.42$ [s, 9H, C $\left.\left(\mathrm{CH}_{3}\right)_{3}\right]$.

${ }^{13} \mathbf{C}$-NMR $\left(126 \mathrm{MHz}, \mathrm{CDCl}_{3}, 303 \mathrm{~K}\right): \delta(\mathrm{ppm})=178.0$ (s, CO), 175.7 (s, COO), 146.6 (s, C-4a), 144.1 (s, C-2), 140.4 (s, C-3), 135.8 (s, C-5a), 133.4 (d, C-6), 130.3 (d, C-8), 128.5 (s, C-8a), 128.4 (d, C-1), 127.8 (d, C-7), 127.0 (s, C-9a), 126.3 (d, C-5), 122.1 (d, $\mathrm{C}-4), 39.5$ [s, $\left.\mathrm{C}\left(\mathrm{CH}_{3}\right)_{3}\right], 27.14(\mathrm{q}, \mathrm{CH} 3)$.

IR (ATR): $v\left(\mathrm{~cm}^{-1}\right)=1759,1636,1604,1553,1343,1212,1099,929,753,743$.

MS (EI, $70 \mathrm{eV}): m / z(\%)=357(18)\left[\mathrm{M}^{+}\right], 309$ (14), 294 (24), $273(73)\left[\mathrm{M}-\mathrm{CO}_{2} \mathrm{tBu}\right]^{+}$, 227 (12) $\left[\mathrm{M}-\mathrm{CO}_{2} \mathrm{tBu}-\mathrm{NO}_{2}\right]^{+}, 171(9), 85(13)\left[\mathrm{CO}_{2} \mathrm{tBu}^{+}\right], 57(100)\left[\mathrm{tBu}^{+}\right]$.

HRMS (EI) $\left(\mathrm{C}_{18} \mathrm{H}_{15} \mathrm{NO}_{2} \mathrm{~S}\right)$ : calc.: 357.0665 ; found: 357.0664 
2-(tert-Butyl)-10H-thioxantheno[2,3-d]oxazol-10-one (7)<smiles>[13CH3]Oc1cc2sc3ccccc3c(=O)c2cc1[N+](=O)[O-]</smiles>

1. $\mathrm{SnCl}_{2}$

(THF) $66^{\circ} \mathrm{C}$

2. $\mathrm{SOCl}_{2}$, pyr

(PhH) $80{ }^{\circ} \mathrm{C}$

$32 \%$

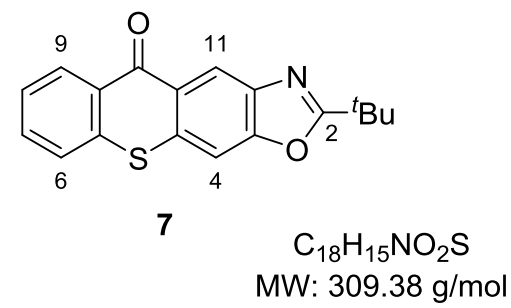

A solution of pivaloate $\mathbf{S 1 9}$ (910 $\mathrm{mg}, 2.55 \mathrm{mmol}, 1.00$ eq.) and dry tin(II) chloride (2.41 g, $12.7 \mathrm{mmol}, 5.00$ eq.) in anhydrous tetrahydrofuran $(50 \mathrm{~mL}, c=$ $50 \mathrm{mmol} / \mathrm{L}$ ) was heated to $70{ }^{\circ} \mathrm{C}$ for $20 \mathrm{~h}$. The mixture was allowed to cool to room temperature and water $(35 \mathrm{~mL})$ was added. The resulting suspension was repeatedly extracted with chloroform until the product cannot be detected in the extract (TLC). The combined organic layers were filtered over celite. The solvent was removed in vacuo to yield $1.46 \mathrm{~g}$ of crude material which was used without further purification.

A solution of the crude product and pyridine $(1.33 \mathrm{~mL}, 16.5 \mathrm{mmol}, 6.50$ eq. $)$ in dry benzene $(17 \mathrm{~mL}, c=0.15 \mathrm{~mol} / \mathrm{L})$ was treated dropwise with thionyl chloride ( $0.55 \mathrm{~mL}, 7.60 \mathrm{mmol}, 3.00$ eq.) and heated to $90{ }^{\circ} \mathrm{C}$ for $20 \mathrm{~h}$. The solution was allowed to cool to room temperature and some silica gel was added carefully. The solvent was removed in vacuo and the mixture was submitted to purification by column chromatography (silica gel, dichloromethane/diethyl ether $=100: 0 \rightarrow 95: 5$ ). Further purification by flash column chromatography (silica gel, hexane/ethyl acetate/dichloromethane $=8: 1: 1)$ gave the pure pure benzoxazole $7(249 \mathrm{mg}, 0.805 \mathrm{mmol}, 32 \%$ over two steps).

M.p.: $156^{\circ} \mathrm{C}$.

TLC: $R_{\mathrm{f}}=0.50$ (cylohexane:ethyl acetate, $8: 2, \mathrm{UV}$ ).

${ }^{1}$ H-NMR $\left(500 \mathrm{MHz}, \mathrm{CDCl}_{3}, 303 \mathrm{~K}\right): \delta(\mathrm{ppm})=8.98(\mathrm{~s}, 1 \mathrm{H}, \mathrm{C}-11-\mathrm{H}), 8.64\left(\mathrm{dd},{ }^{3} J=8.0\right.$ $\left.\mathrm{Hz},{ }^{4} J=1.4 \mathrm{~Hz}, 1 \mathrm{H}, \mathrm{C}-9-\mathrm{H}\right), 7.67$ (s, 1H, C-4-H), 7.66-7.57 (m, 2H, C-6-H, C-8-H), 7.50 (ddd, $\left.{ }^{3} \mathrm{~J}=8.2,7.0,{ }^{4} \mathrm{~J}=1.3 \mathrm{~Hz}, 1 \mathrm{H}, \mathrm{C}-7-\mathrm{H}\right), 1.53\left[\mathrm{~s}, 9 \mathrm{H}, \mathrm{C}\left(\mathrm{CH}_{3}\right)_{3}\right]$.

${ }^{13} \mathrm{C}$-NMR $\left(126 \mathrm{MHz}, \mathrm{CDCl}_{3}, 303 \mathrm{~K}\right): \delta(\mathrm{ppm})=179.9$ (s, CO), 175.5 (s, C-2), 153.5 (s, C-3a), 141.3 (s, C-11a), 137.2 (s, C-5a), 134.0 (s, C-10a), 132.4 (d, C-7), 130.1 (d, C-9), 128.8 (s, C-9a), 126.5 (s, C-4a), 126.5 (d, C-8), 125.8 (d, C-6), 121.9 (d, C-11), 106.7 (d, C-4) , $34.6\left[\mathrm{~s}, \mathrm{C}\left(\mathrm{CH}_{3}\right)_{3}\right], 28.6\left(\mathrm{q}, \mathrm{CH}_{3}\right)$.

IR (ATR): $v\left(\mathrm{~cm}^{-1}\right)=2973,1635,1617,1589,1436,1412,1289,1246,1112,1101$, 1043, 1032, 915, 860, 809, 744.

MS (EI, $70 \mathrm{eV}): m / z(\%)=309(72)\left[\mathrm{M}^{+}\right], 294(100)\left[\mathrm{M}-\mathrm{CH}_{3}\right]^{+}, 267(14)\left[\mathrm{M}-\mathrm{CO}-\mathrm{CH}_{3}\right]^{+}$, $253(7)[\mathrm{M}-t \mathrm{Bu}]^{+}, 225$ (12) $[\mathrm{M}-\mathrm{CO}-t \mathrm{Bu}]^{+}$.

HRMS (EI) $\left(\mathrm{C}_{18} \mathrm{H}_{15} \mathrm{NO}_{2} \mathrm{~S}\right)$ : calc.: 309.0818 ; found: 309.0821 


\section{Competition Experiment}

Prior to the experiment the GC calibration parameters were determined for every compound.

Dodecane: $\quad y=78.12904 x+0.34781$

Quinolone S20: $\quad y=67.42409 x-1.17052$

Product 4f: $\quad y=92.76575 x-0.25822$

Product 4o: $\quad y=107.6437 x+0.38640$

Thioxanthone 7: $\quad y=174.4212 x-1.56069$

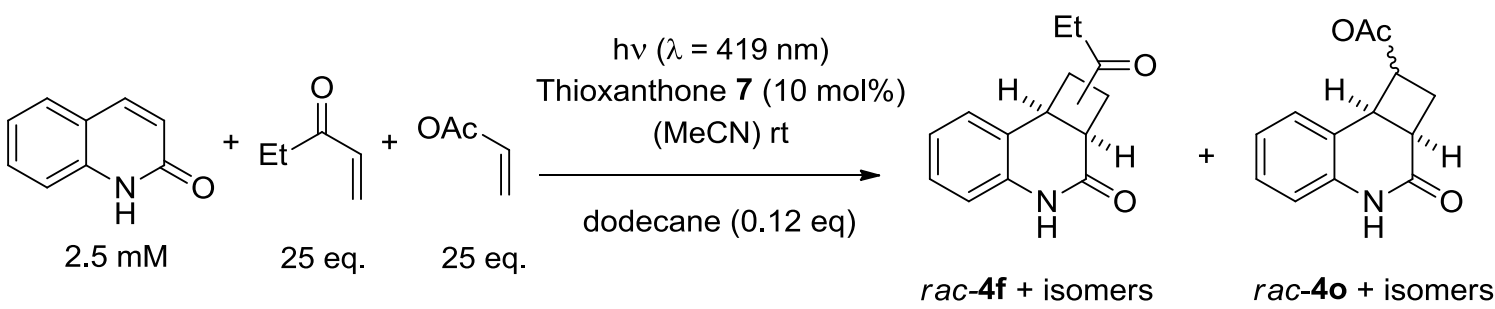

In the experiment 2(1H)-quinolone (S20) $(7.20 \mathrm{mg}, 50.0 \mu \mathrm{mol}, 1.00$ eq.) and thioxanthone 7 ( $1.56 \mathrm{mg}, 5.04 \mu \mathrm{mol}, 0.10$ eq.) were dissolved in degassed acetonitrile $(20 \mathrm{~mL}, c=2.5 \mathrm{mmol} / \mathrm{L})$. Dodecane was used as internal standard $(1.36 \mu \mathrm{L}, 5.99 \mu \mathrm{mol}$, 0.12 eq.). Subsequently, pent-1-en-3-one (123 $\mu \mathrm{l}, 1.24 \mathrm{mmol}, 25$ eq.) and vinyl acetate (114 $\mu 1,1.24 \mathrm{mmol}, 25$ eq.) were added and the solution was irradiated at $419 \mathrm{~nm}$ at room temperature. Aliquots of $100 \mu \mathrm{L}$ were taken from the reaction mixture after 0,10 , 20, 30, 45, 60, 75, 90 and 120 mins reaction time, and measured without dilution. GC analysis was carried using the following temperature profile: $60{ }^{\circ} \mathrm{C}$ (hold $3 \mathrm{~min}$ ), then $15^{\circ} \mathrm{C} / \mathrm{min}$ to $250^{\circ} \mathrm{C}$ (hold $3 \mathrm{~min}$ ), then $40^{\circ} \mathrm{C} / \mathrm{min}$ to $300{ }^{\circ} \mathrm{C}$ (hold $5 \mathrm{~min}$ ).

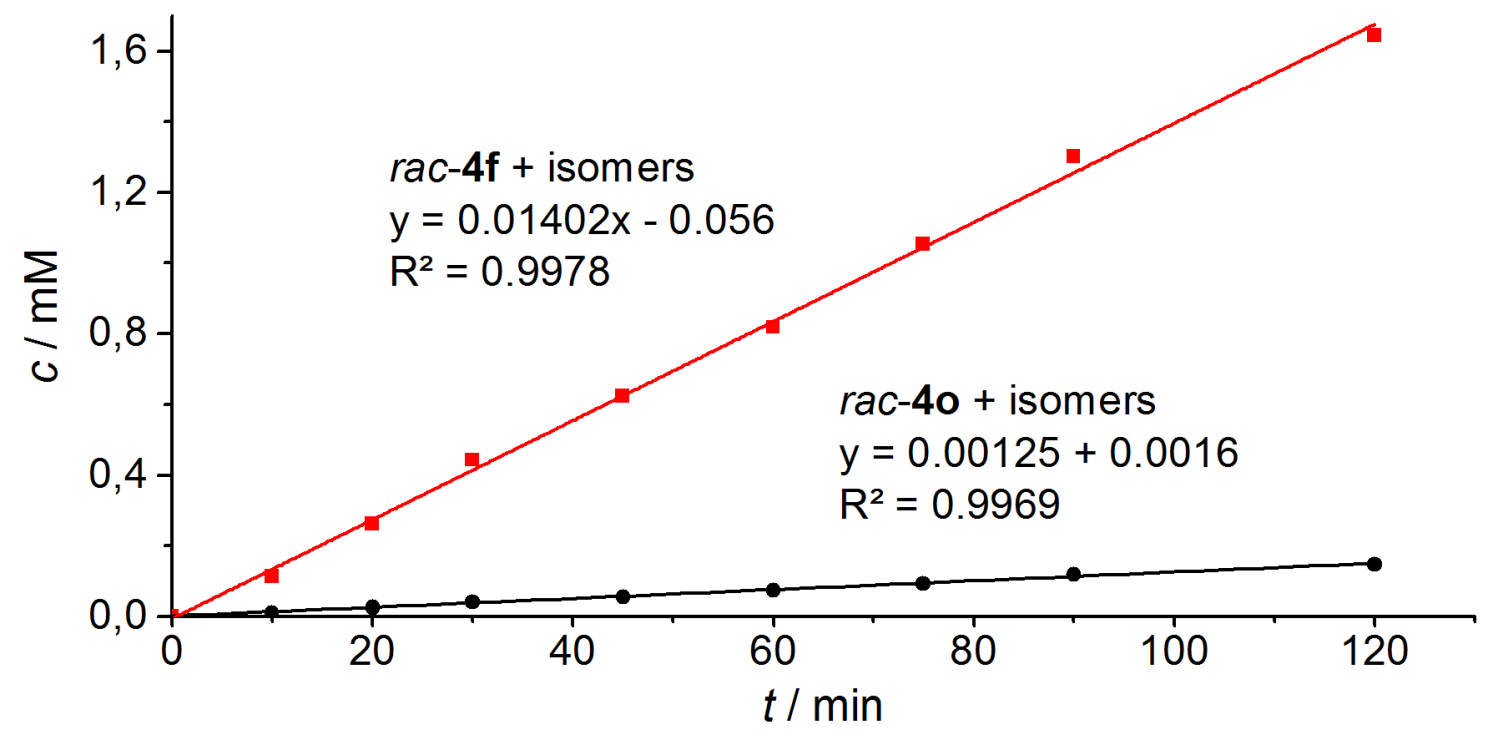




\section{Areas measured by GC}

Table S1: GC data (area) for Quinolone S20 product $\mathbf{4 f}$ (+isomers) and product $4 \mathbf{0}$ (+isomers) at different reaction times. The conversion of quinolone $\mathbf{S 2 0}$ was calculated as the change of the area of $\mathbf{S 2 0}$.

\begin{tabular}{c|ccccc}
\hline \hline Time [min] & Dodecane & Quinolone S20 & $\begin{array}{c}\text { Product 4f } \\
\text { + isomers }\end{array}$ & $\begin{array}{c}\text { Product 4o } \\
\text { + isomers }\end{array}$ & $\begin{array}{c}\text { Conversion } \\
(\%)\end{array}$ \\
\hline \hline 0 & 23.6 & 158.7 & 0.0 & 0.0 & 0.0 \\
10 & 22.1 & 126.7 & 1.7 & 13.4 & 14.8 \\
20 & 21.1 & 107.8 & 3.4 & 29.5 & 24.2 \\
30 & 24.2 & 106.4 & 6.3 & 57.2 & 34.7 \\
45 & 23.6 & 84.7 & 8.4 & 78.6 & 46.8 \\
60 & 21.5 & 66.2 & 10.1 & 94.0 & 54.3 \\
75 & 23.6 & 53.8 & 13.8 & 132.7 & 66.2 \\
90 & 19.5 & 38.8 & 14.4 & 135.5 & 70.5 \\
120 & 18.0 & 21.8 & 16.6 & 158.0 & 82.0 \\
\hline \hline
\end{tabular}

\section{Concentrations of starting material S20 and products $4 \mathbf{f} / 40$}

Table S2: Calculated concentrations for quinolone S20, product $\mathbf{4 f}$ (+isomers) and product 40 (+isomers).

\begin{tabular}{c|ccccccccc}
\hline \hline Time [min] & $\mathbf{0}$ & $\mathbf{1 0}$ & $\mathbf{2 0}$ & $\mathbf{3 0}$ & $\mathbf{4 5}$ & $\mathbf{6 0}$ & $\mathbf{7 5}$ & $\mathbf{9 0}$ & $\mathbf{1 2 0}$ \\
\hline \hline Quinolone S20 (mM) & 2.74 & 2.33 & 2.08 & 1.79 & 1.46 & 1.25 & 0.93 & 0.81 & 0.49 \\
$\mathbf{4 f}+$ isomers $(\mathrm{mM})$ & 0.00 & 0.11 & 0.26 & 0.44 & 0.62 & 0.82 & 1.05 & 1.30 & 1.62 \\
$\mathbf{4 0}+$ isomers $(\mathrm{mM})$ & 0.00 & 0.01 & 0.03 & 0.04 & 0.06 & 0.08 & 0.09 & 0.12 & 0.15 \\
\hline \hline
\end{tabular}

\section{Competition Experiment Using Catalyst 3}

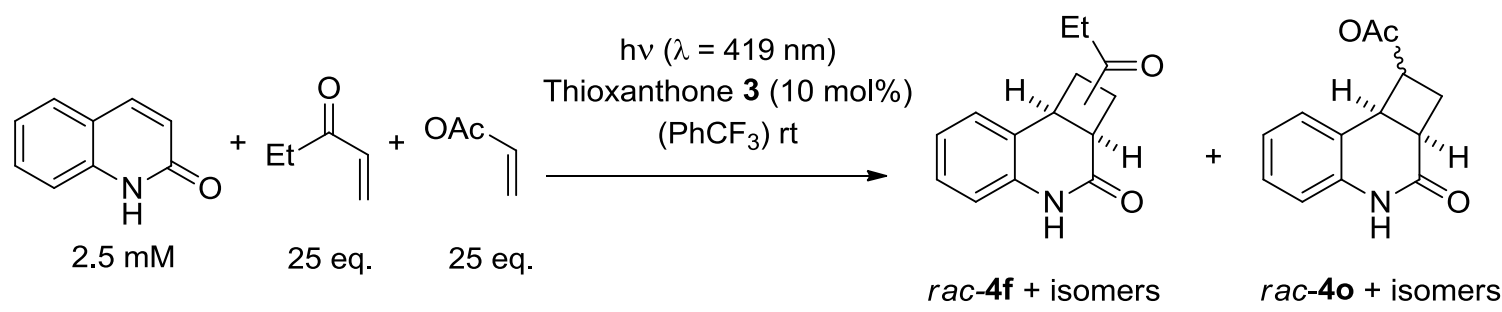

In the experiment 2(1H)-quinolone (S20) $(3.65 \mathrm{mg}, 25.0 \mu \mathrm{mol}, 1.00 \mathrm{eq}$.$) and$ enantiomerically pure $3(1.08 \mathrm{mg}, 2.50 \mu \mathrm{mol}, 0.10$ eq.) were dissolved in degassed $\alpha, \alpha, \alpha$-trifluorotoluene $(10 \mathrm{~mL}, c=2.5 \mathrm{mmol} / \mathrm{L})$. Subsequently, pent-1-en-3-one $(62.2$ $\mu 1,0.63 \mathrm{mmol}, 25$ eq.) and vinyl acetate $(57.6 \mu \mathrm{l}, 0.63 \mathrm{mmol}, 25$ eq.) were added and the solution was irradiated at $419 \mathrm{~nm}$ at room temperature for $30 \mathrm{~min}$. The reaction was 
stopped and the reaction mixture was analyzed by GLC after removal of the catalyst. GC analysis was carried using the following temperature profile: $60{ }^{\circ} \mathrm{C}$ (hold $3 \mathrm{~min}$ ), then $15^{\circ} \mathrm{C} / \mathrm{min}$ to $250^{\circ} \mathrm{C}$ (hold $3 \mathrm{~min}$ ), then $40^{\circ} \mathrm{C} / \mathrm{min}$ to $300{ }^{\circ} \mathrm{C}$ (hold $5 \mathrm{~min}$ ).

Table S3: GC data (area) and calculated concentrations for product $4 \mathbf{f}$ (+isomers) and product 4o (+isomers) after $30 \mathrm{~min}$. The ratio of $\mathrm{k}_{\mathrm{COE}} / \mathrm{k}_{\mathrm{OAc}}$ determined under these conditions was 7.7 .

\begin{tabular}{c|ccccc}
\hline \hline Time [min] & $\begin{array}{c}\text { Product 4f } \\
+ \text { isomers }\end{array}$ & $\begin{array}{c}\text { Product 4o } \\
+ \text { isomers }\end{array}$ & $\begin{array}{c}\mathbf{4 f}+\text { isomers } \\
(\mathrm{mM})\end{array}$ & $\begin{array}{c}\mathbf{4 o}+\text { isomers } \\
(\mathrm{mM})\end{array}$ & $\mathrm{k}_{\text {COEt }} / \mathrm{k}_{\mathrm{OAc}}$ \\
\hline \hline 30 & 73.8 & 12.1 & 0.23 & 0.03 & 7.7
\end{tabular}




\section{Emission Spectra of the Light Sources.}

Emission spectra of the light sources used in this study (Rayonet RPR-4190A) were measured using a USB-4000 spectrometer (OceanOptics Inc. Dunedin, FL, USA; 600 lines grating blazed at $300 \mathrm{~nm}$; bandwidth $200-850 \mathrm{~nm} ; 10 \mu \mathrm{m}$ slit) through a cosine corrector connected with a $400 \mu \mathrm{m}$ fibre to the spectrometer. The light intensity was measured at the lamp centre in a distance of $10 \mathrm{~cm}$ and refers to a calibration of the above described setup against radiometric calibration standards (OceanOptics Inc. Dunedin, FL, USA) traceable to NIST references.

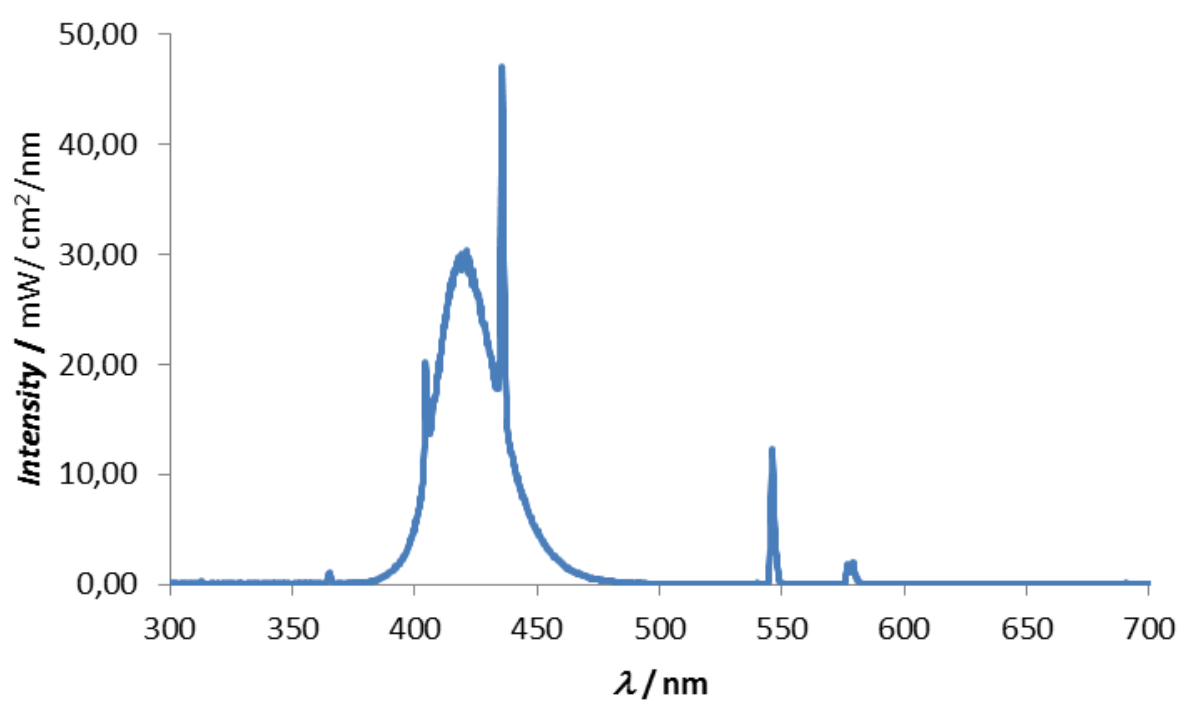




\section{Solar Irradiation Experiment}

Solar irradiation experiments were conducted in the standard low-temperature setup consisting of the irradiation tube in a vaccum-insulated cooling finger. The cooling finger was placed in a surrounding cylinder filled with a slightly acidified $(0.075 \mathrm{~mol} / \mathrm{L}$ $\mathrm{HCl}$ ) iron(III) sulfate solution ( $\mathrm{c}=10.5 \mathrm{~g} / \mathrm{L}$ ) to cut off the UV-content of the incident light. ${ }^{1}$ Both, cooling finger and phototube were held in place by spacers to result in a stable, concentrical setup; the resulting thickness of the filter solution was $10 \mathrm{~mm}$.

The irradiation setup was placed in the focus of a parabolic mirror (see Figure S5). During prolonged irradiation, the complete device was turned and tilted, to follow the course of the sun.

Figure S5: Apparatus for solar irradiation and UV-Vis transmission spectra of various concentrations of a iron(III) sulfate solution (10 mm optical pathlength).
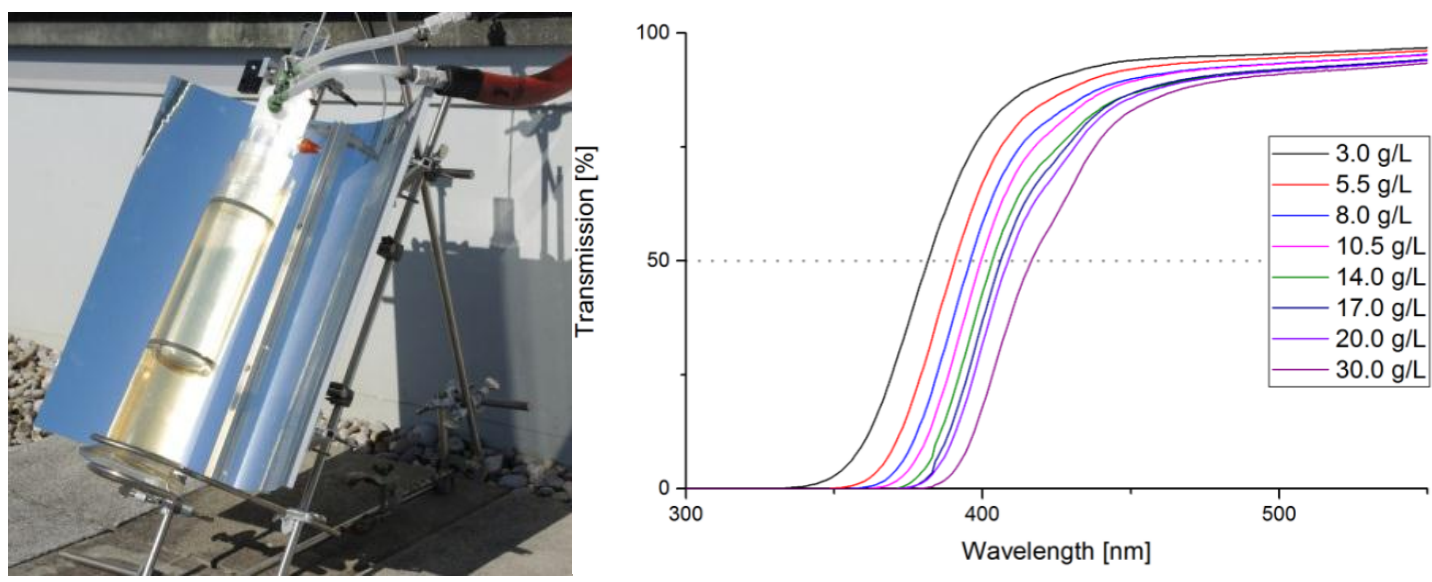

Enantioselective [2+2] Photocycloaddition

2(1H)-Quinolone $(2.9 \mathrm{mg}, 20.0 \mu \mathrm{mol}, 1.00$ eq.) and enantiomerically pure $3(0.9 \mathrm{mg}$, $2.0 \mu \mathrm{mol}, 10 \mathrm{~mol} \%)$ were dissolved in $\alpha, \alpha, \alpha$-trifluorotoluene $(8 \mathrm{~mL}, c=2.5 \mathrm{mmol} / \mathrm{L})$. After degassing the solution, pent-1-en-3-one ( $99 \mu \mathrm{l}, 1.00 \mathrm{mmol}, 50.0$ eq.) was added and the mixture was exposed to sunlight irradiation for $4 \mathrm{~h} 15 \mathrm{~min}$ at $-25{ }^{\circ} \mathrm{C}(21 \%$ conversion). After removing the solvent, purification was achieved by flash column chromatography (silica, pentane/ethyl acetate $7: 3 \rightarrow 1: 1)$. The title compound $\mathbf{4 f}(0.8$ $\mathrm{mg}, 3.5 \mu \mathrm{mol}, 17 \%, 86 \%$ ee, $81 \%$ based on recovered starting material) and remaining starting material $(2.3 \mathrm{mg}, 16.0 \mu \mathrm{mol}, 79 \%)$ were obtained as a colorless solid.

\footnotetext{
${ }^{1}$ The concentration needs to be optimized for different sunlight-intensities; $10.5 \mathrm{~g} / \mathrm{L}$ where used for low intensity sun light (Munich, late October 2015).
} 
9. NMR-Spectra of New Compounds

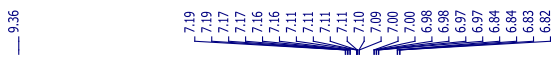

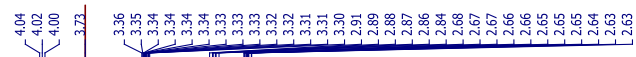<smiles>CCCCC1CCC(=O)Nc2ccccc21</smiles><smiles>C1#CC2C=CC12</smiles>
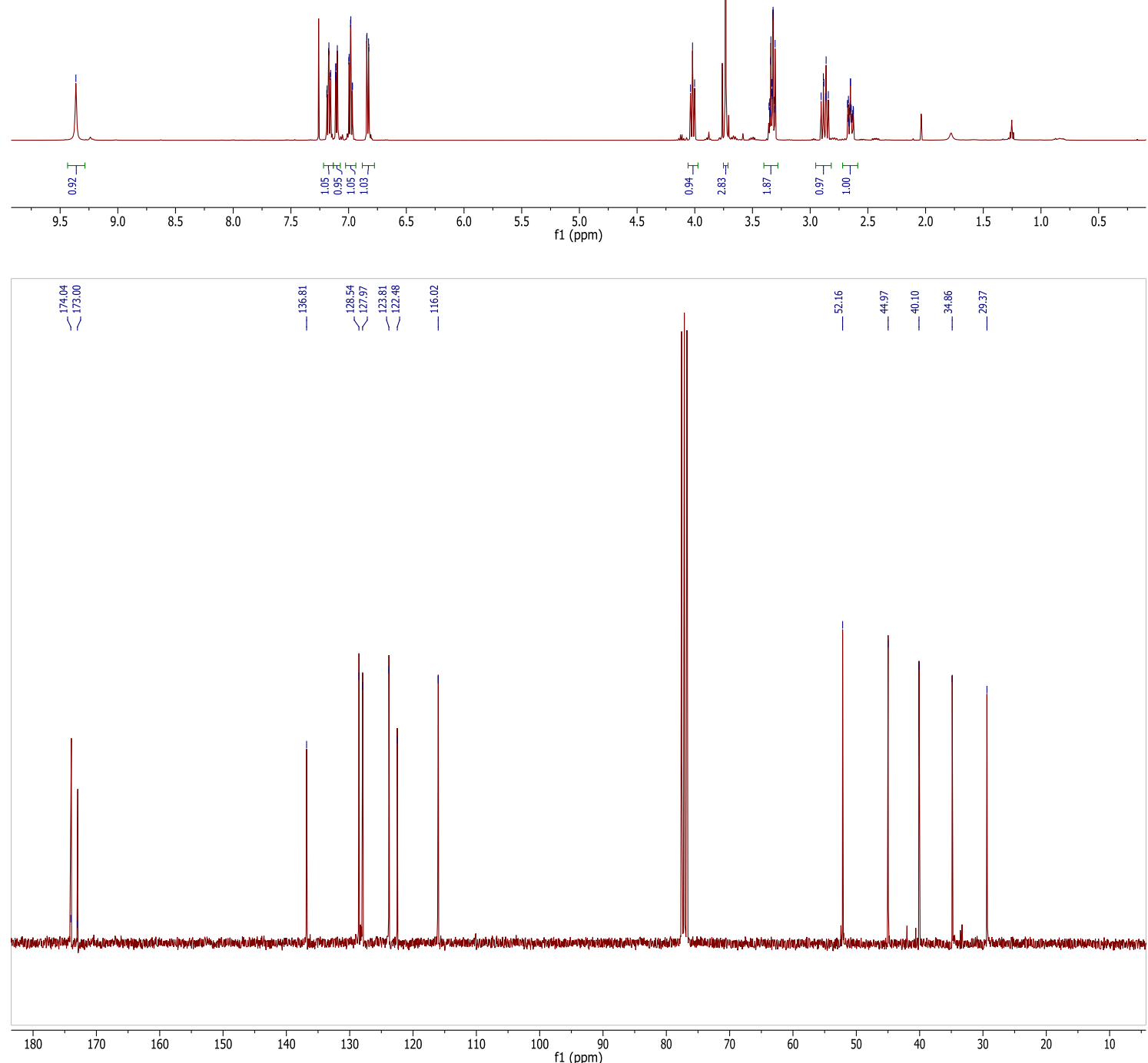

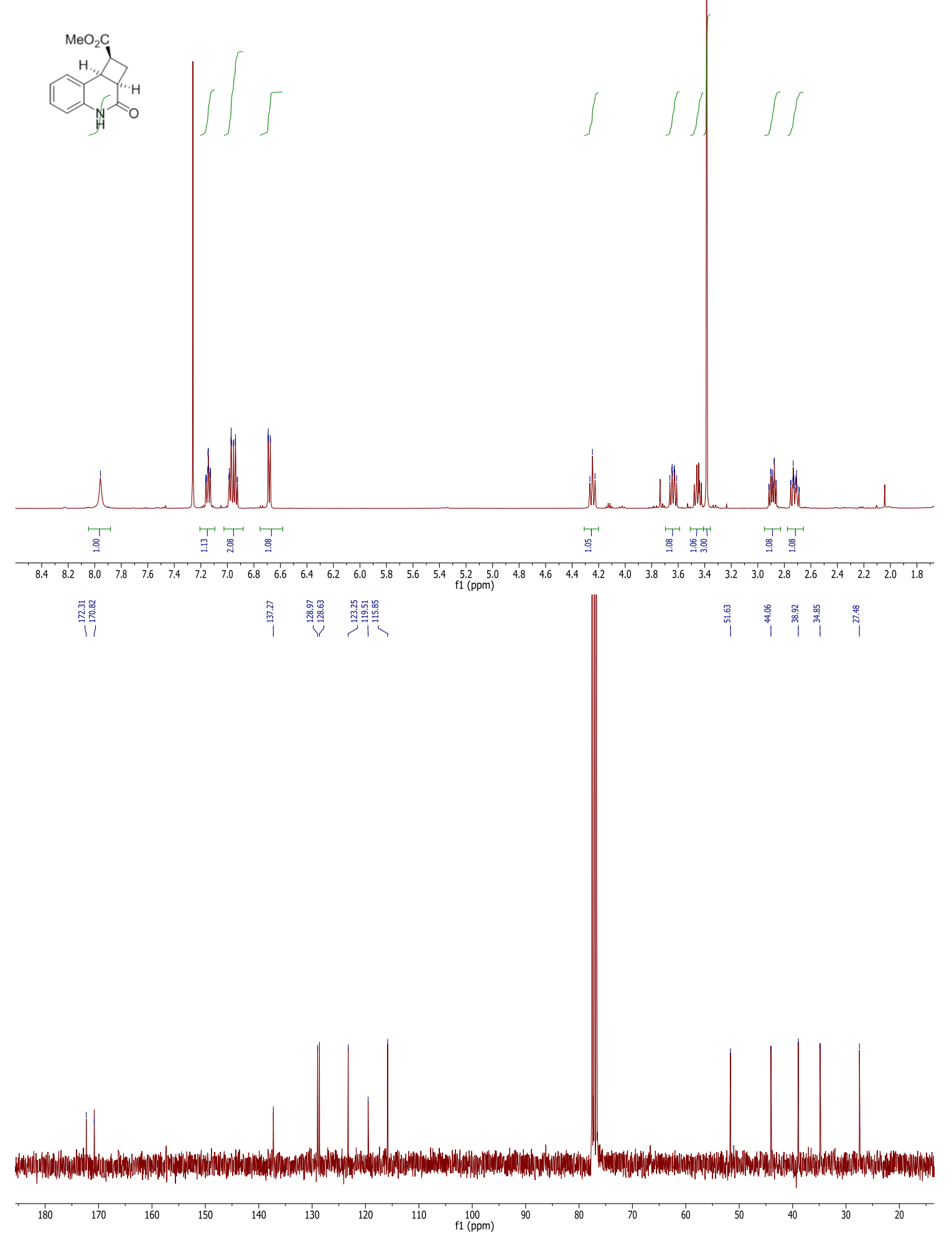


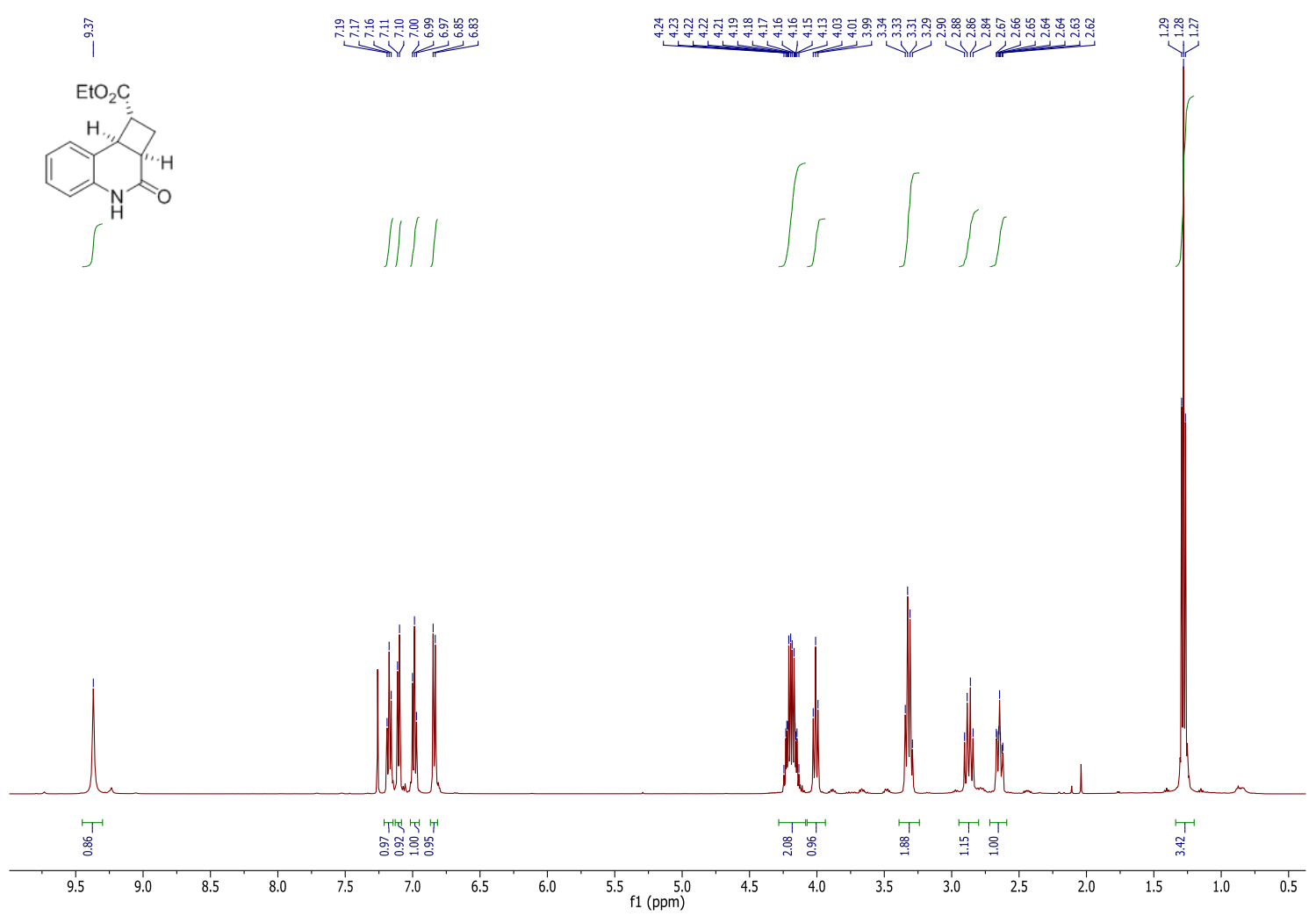

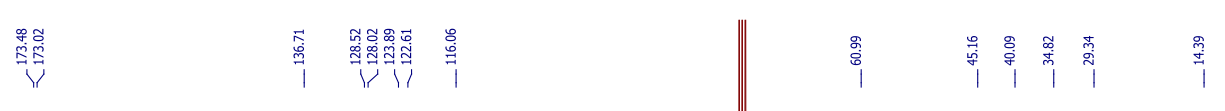

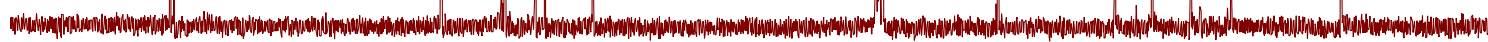

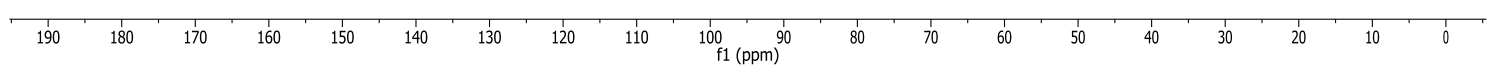



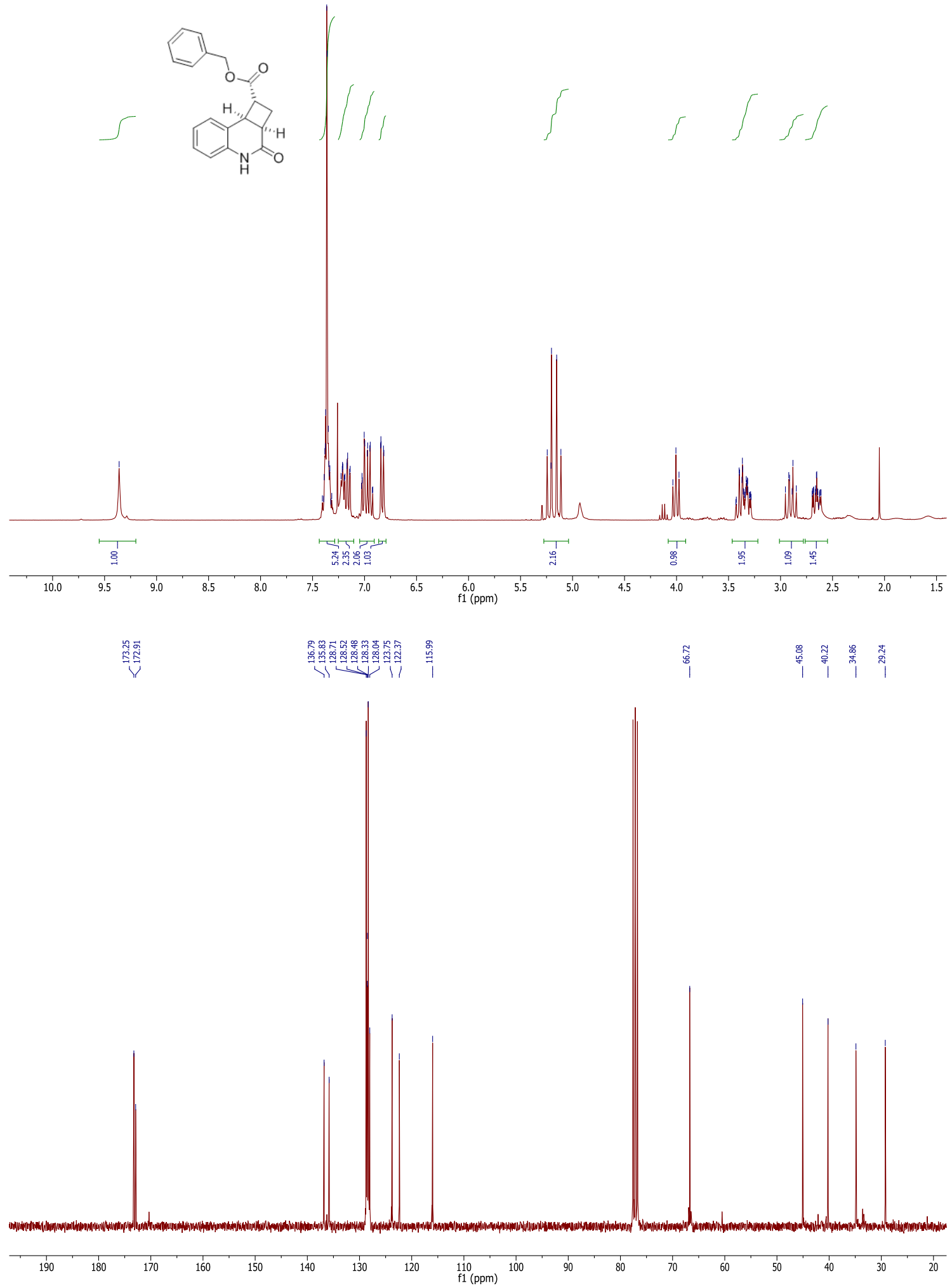

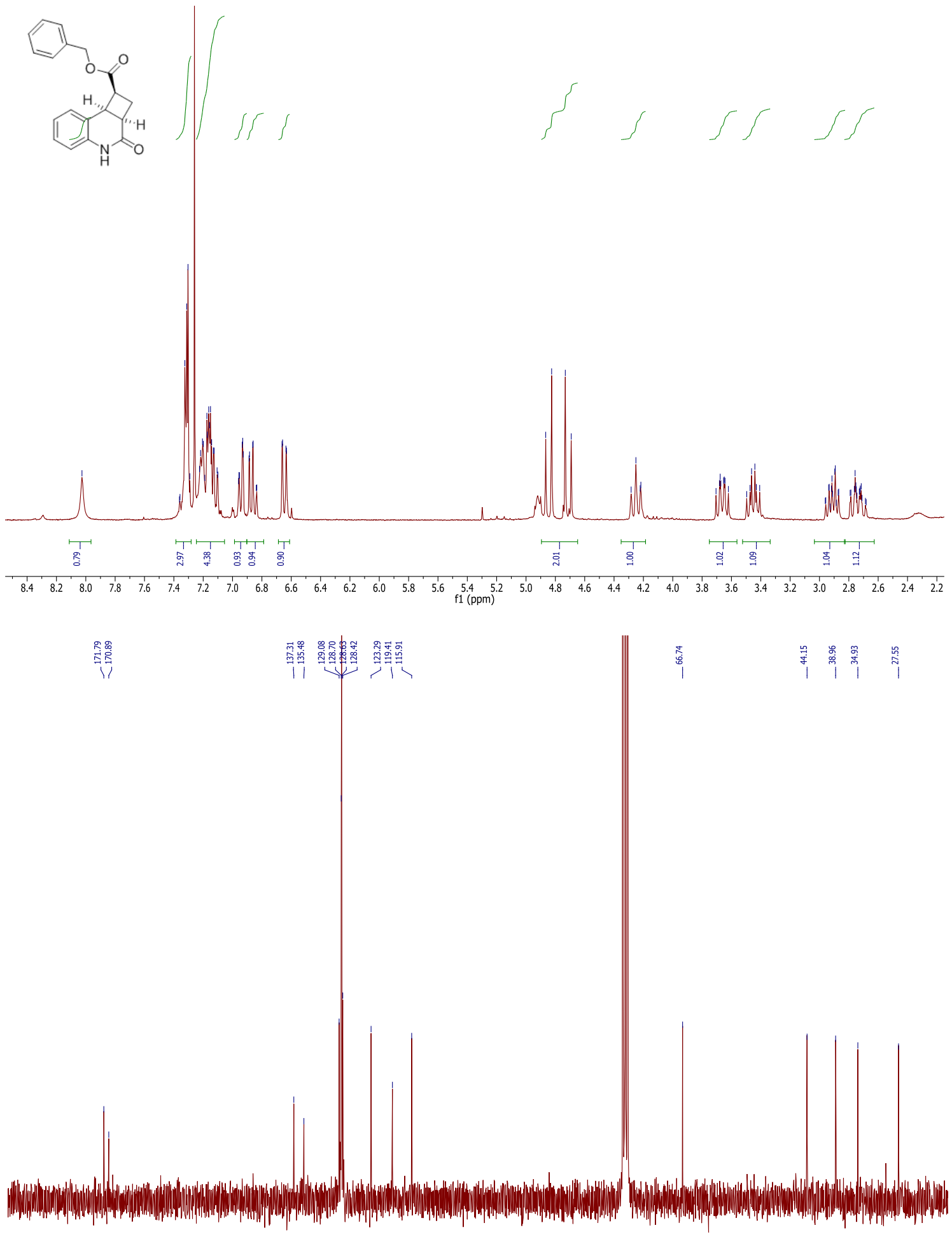

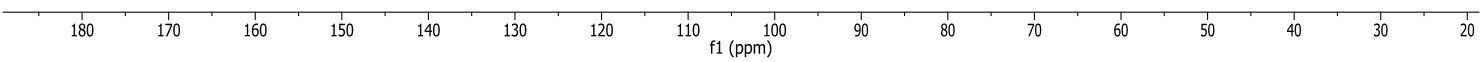




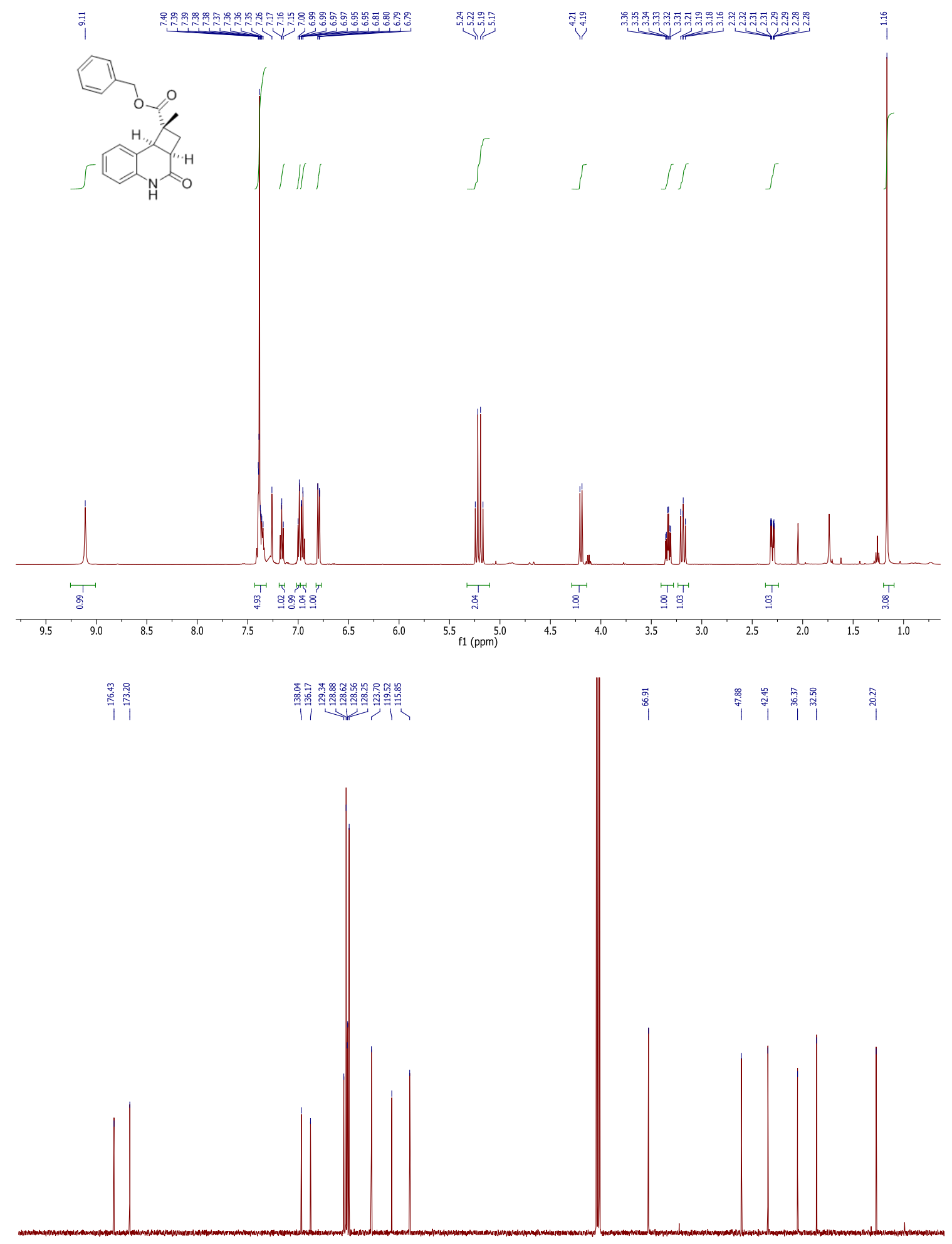

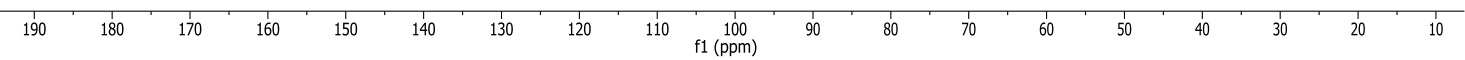




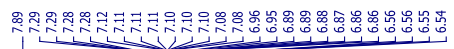

匀

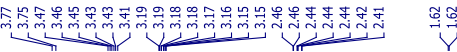
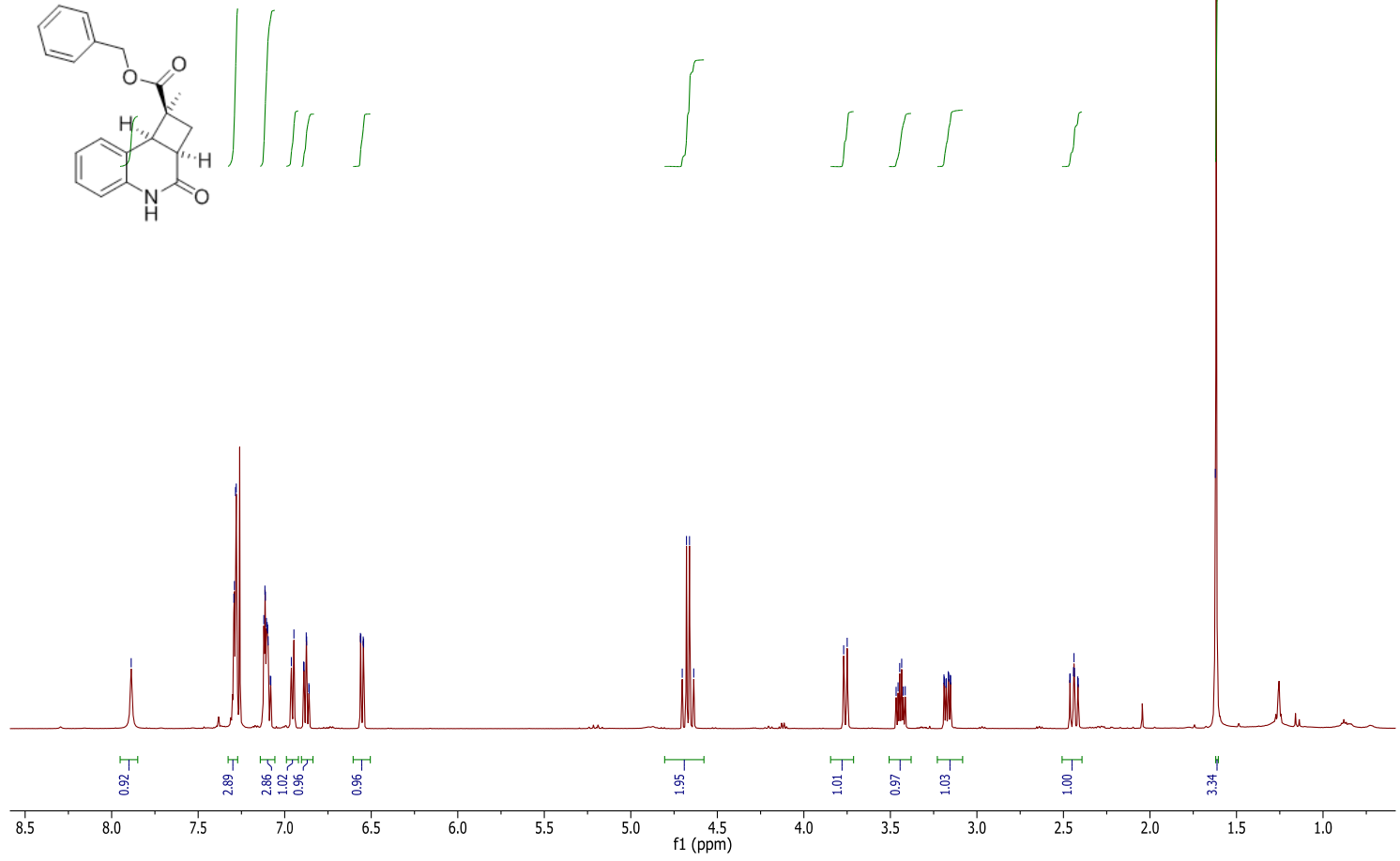

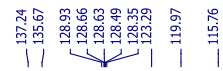

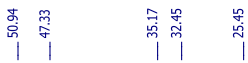

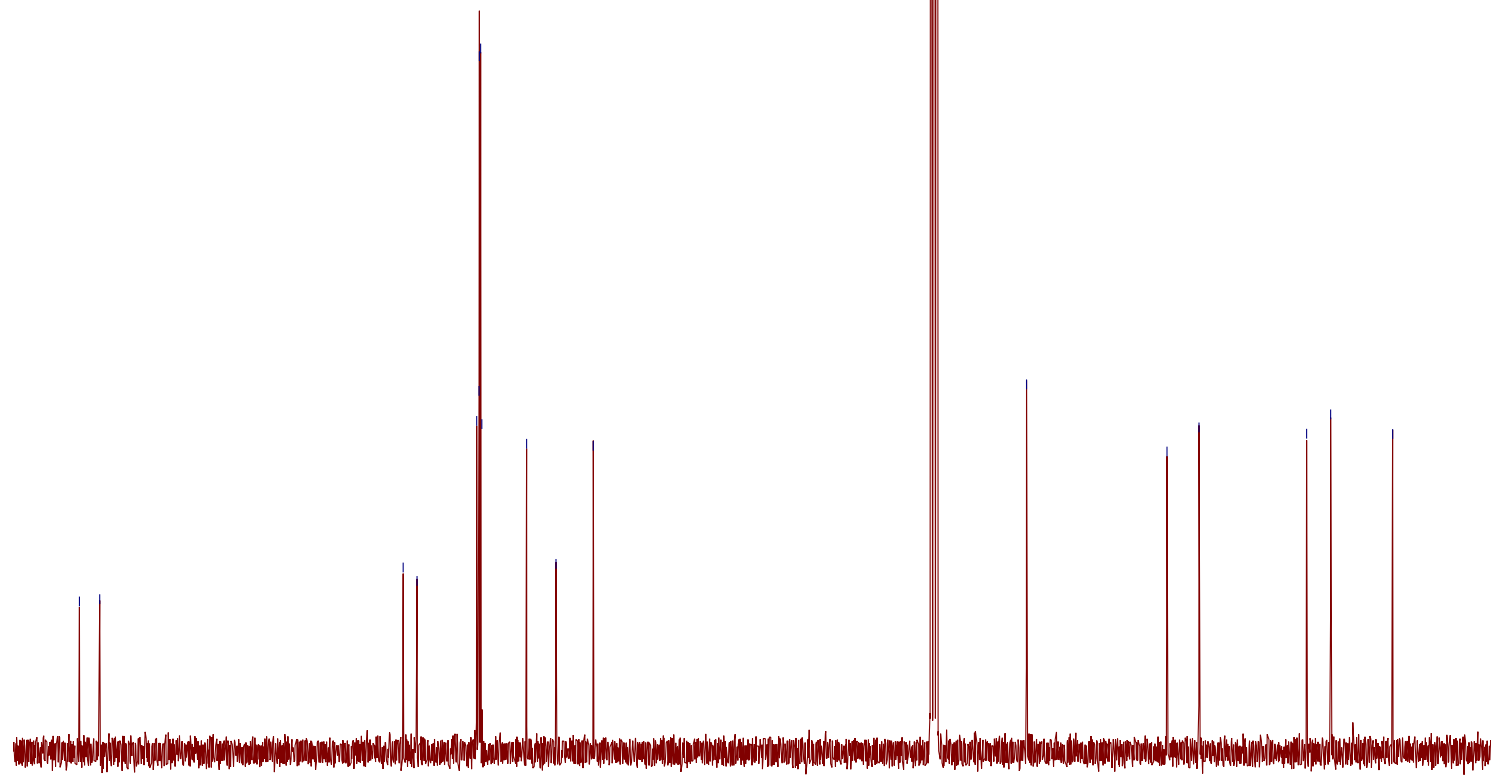

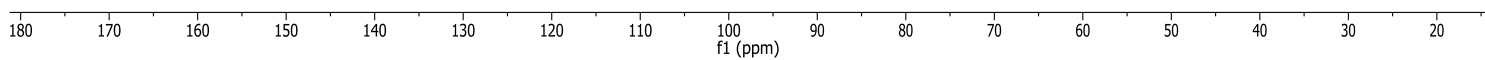



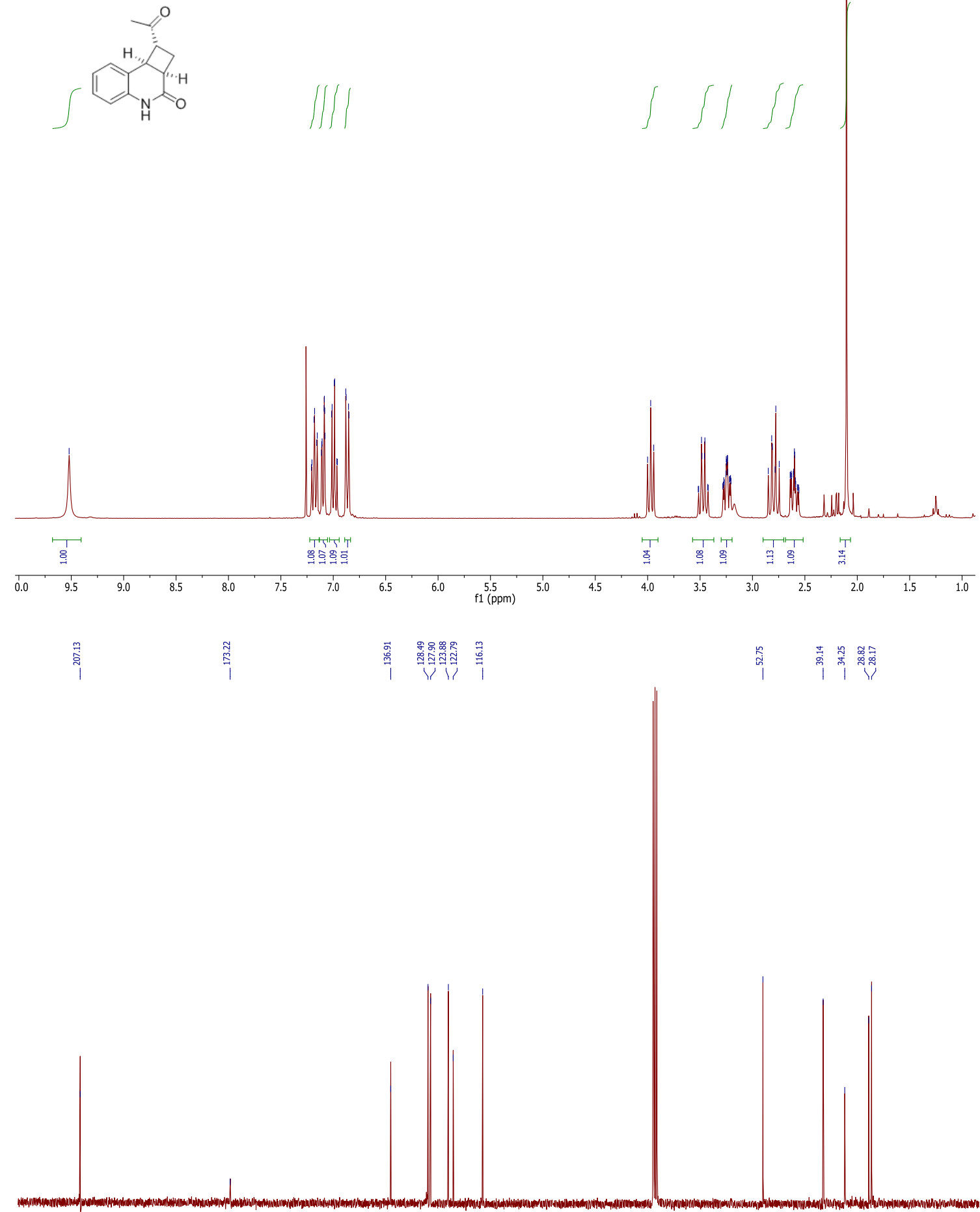

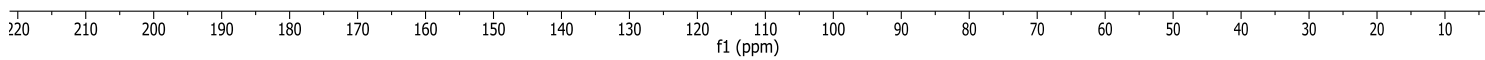




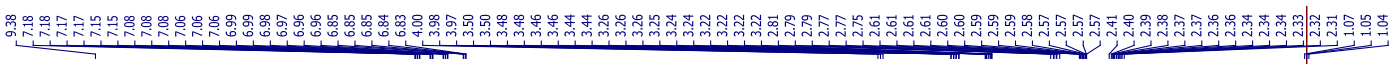

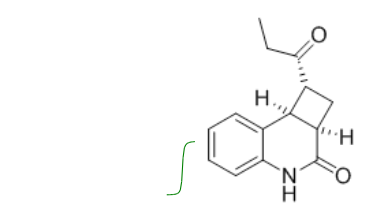

IIII

$\iiint\|\|$
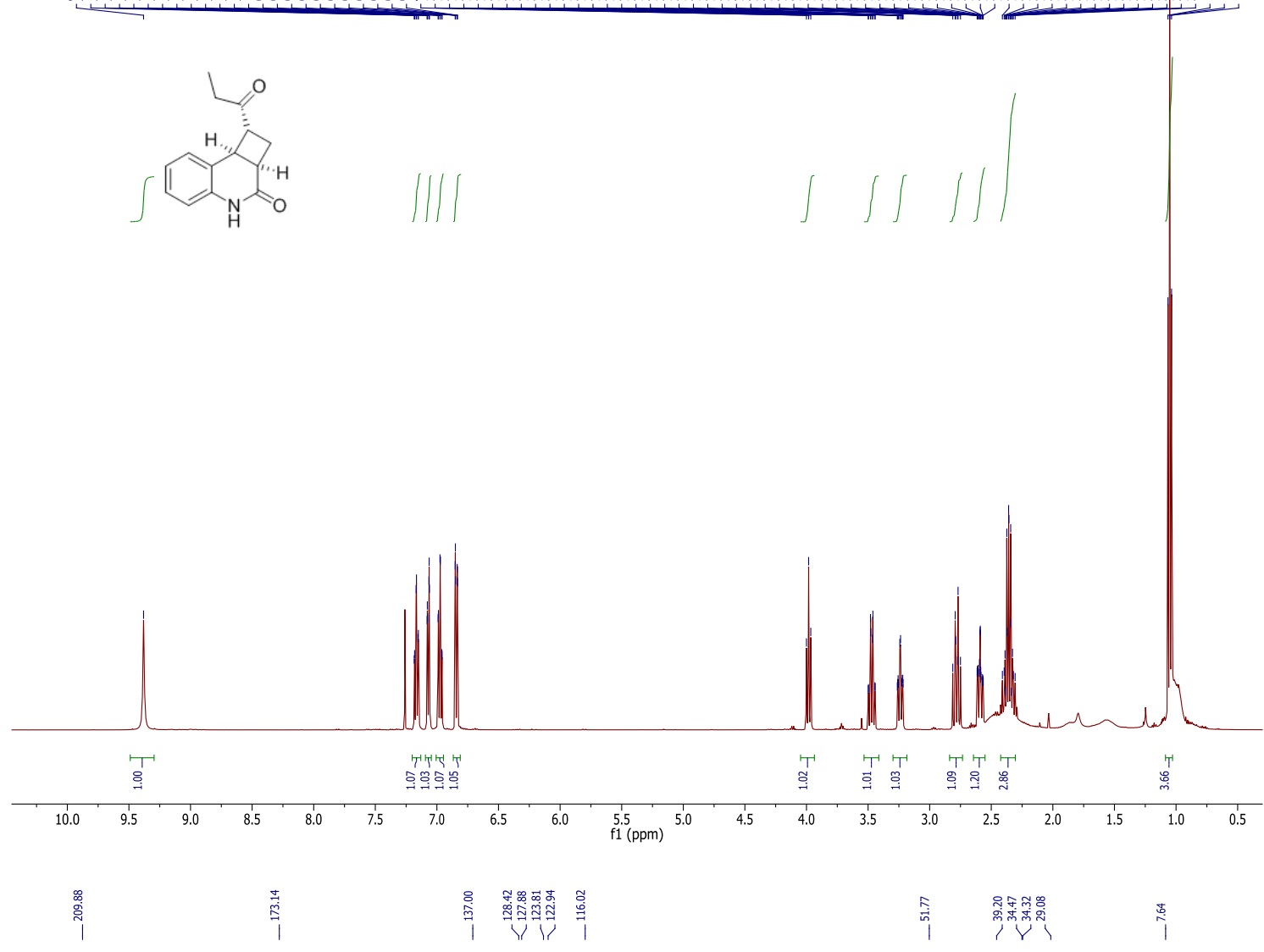

$\stackrel{\text { ¿ }}{\text { i }}$

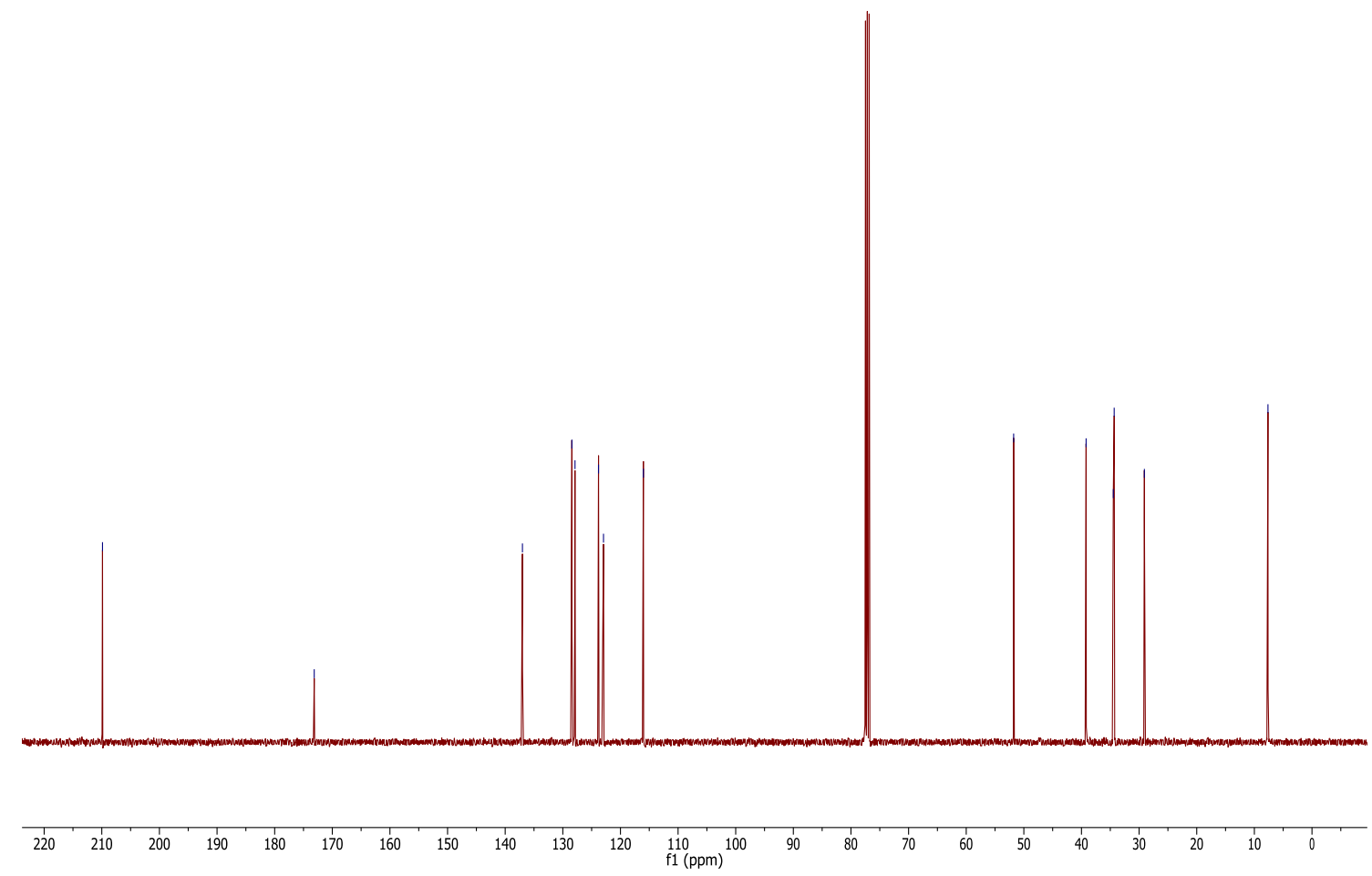

-S58- 

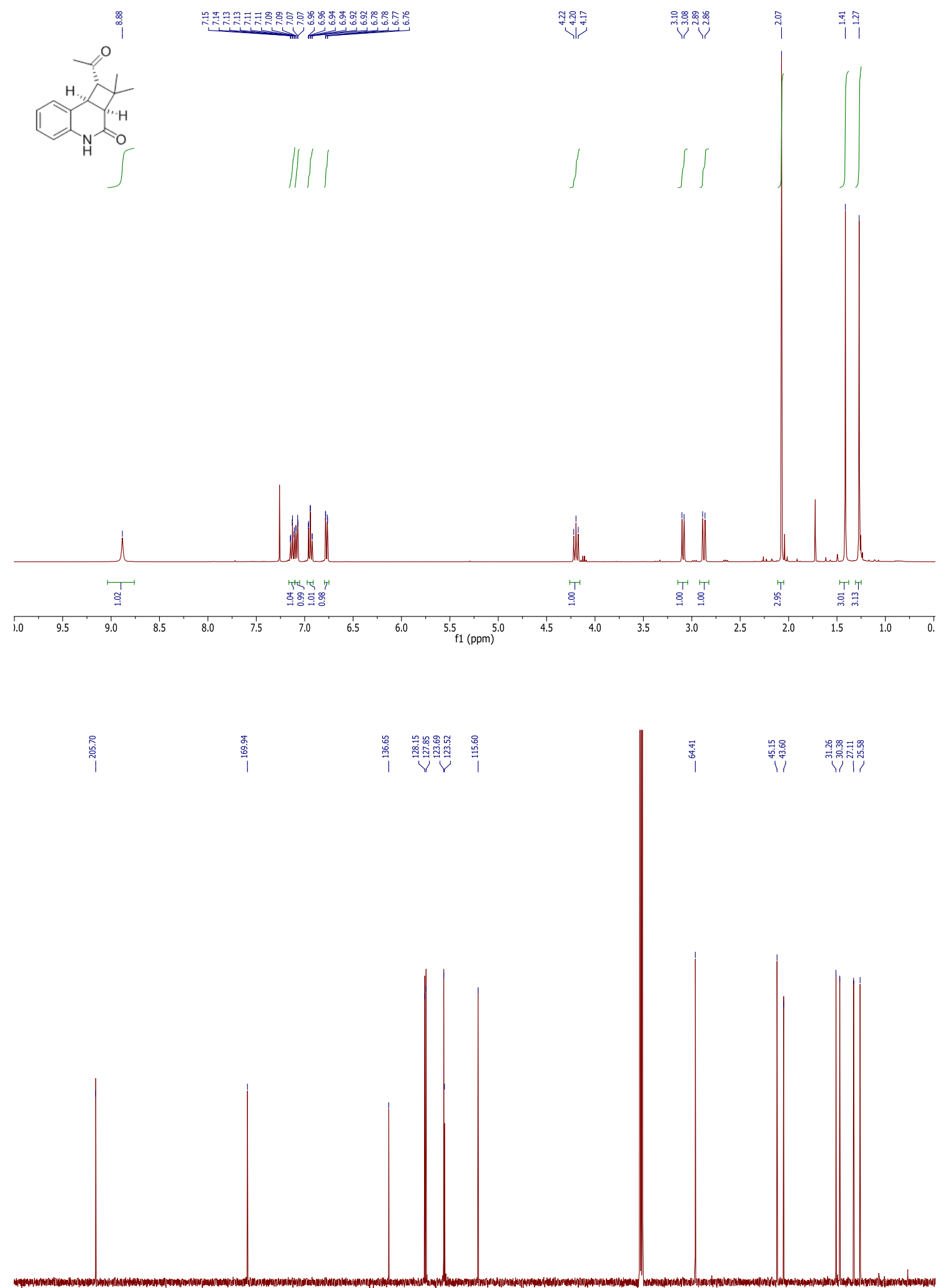

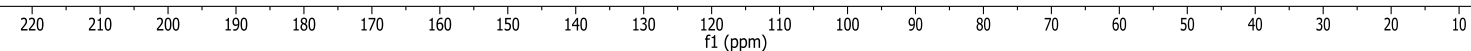


Y

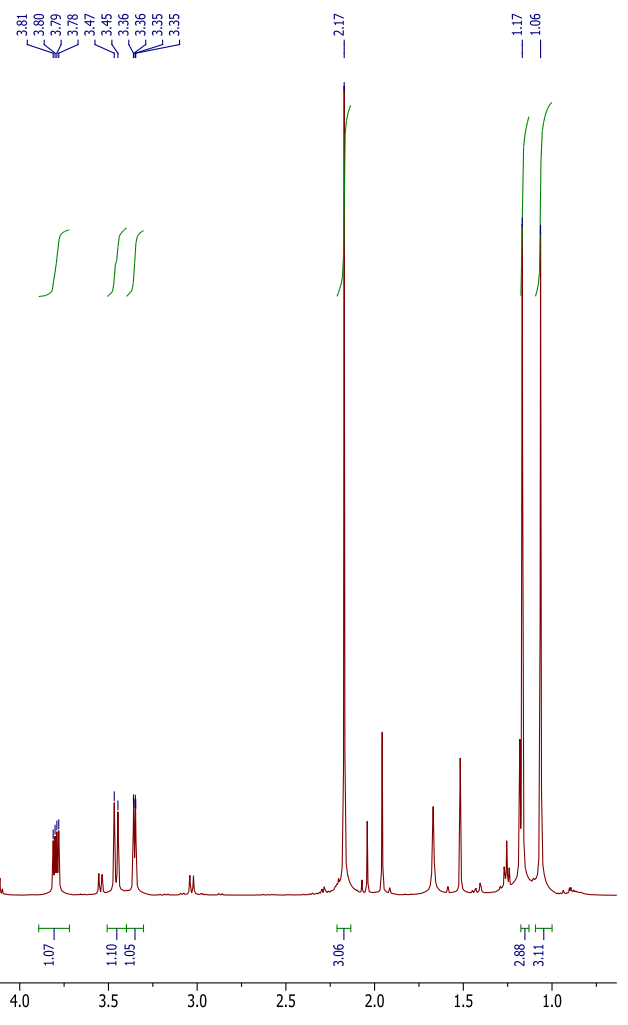
总

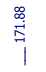

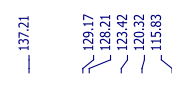
||

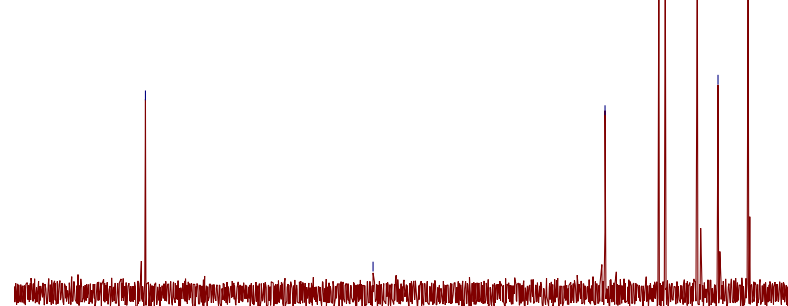

\begin{tabular}{|llllllllllll}
\hline 220 & 210 & 200 & 190 & 180 & 170 & 160 & 150 & 140 & 130 & $\frac{1}{120} 110$ \\
$\mathrm{f} 1(\mathrm{ppm})$ & 110
\end{tabular} 


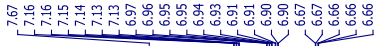<smiles>COC1Nc2ccccc2C1(C)C(C)O</smiles>

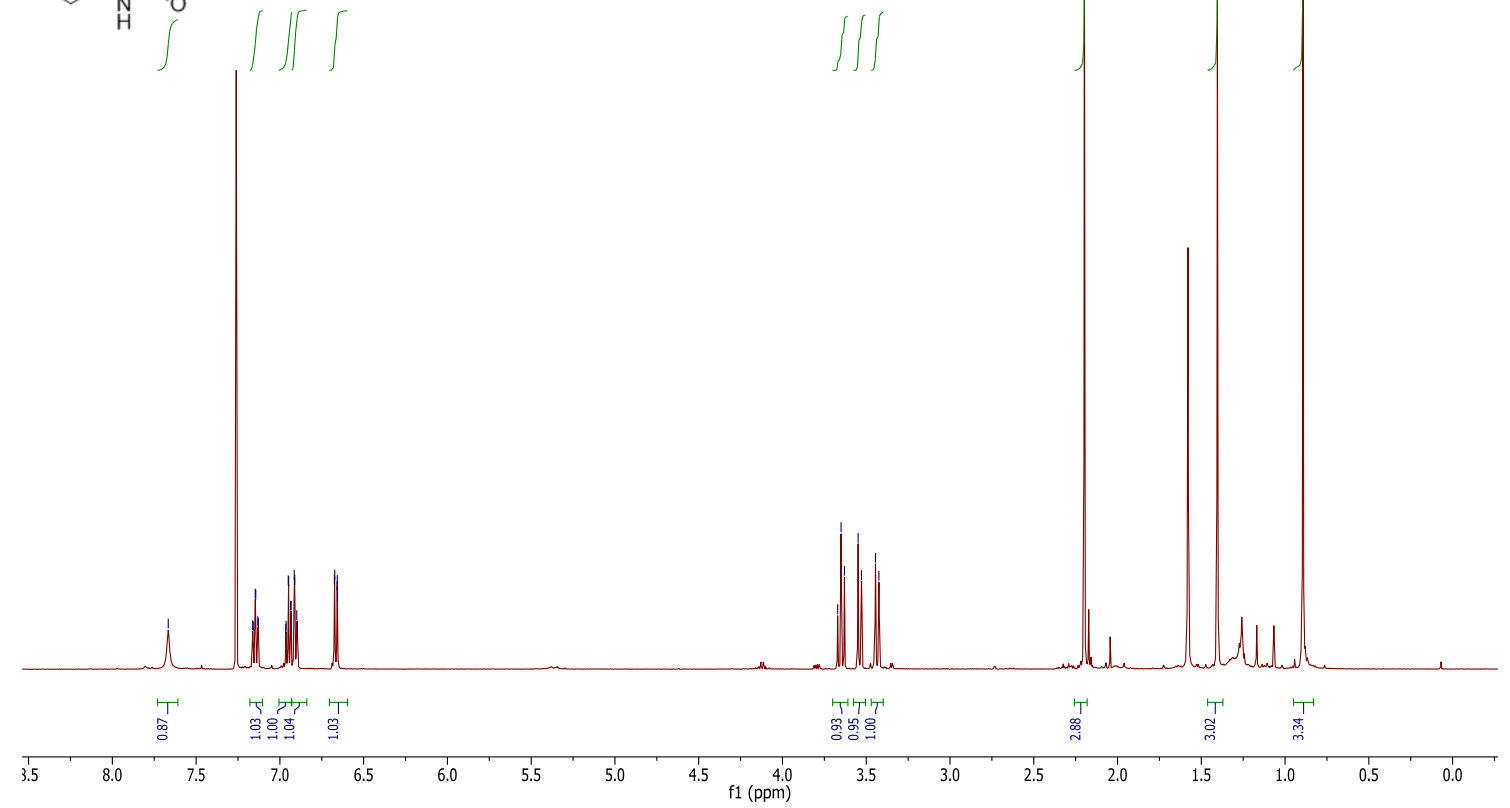

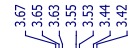

สิ

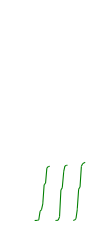

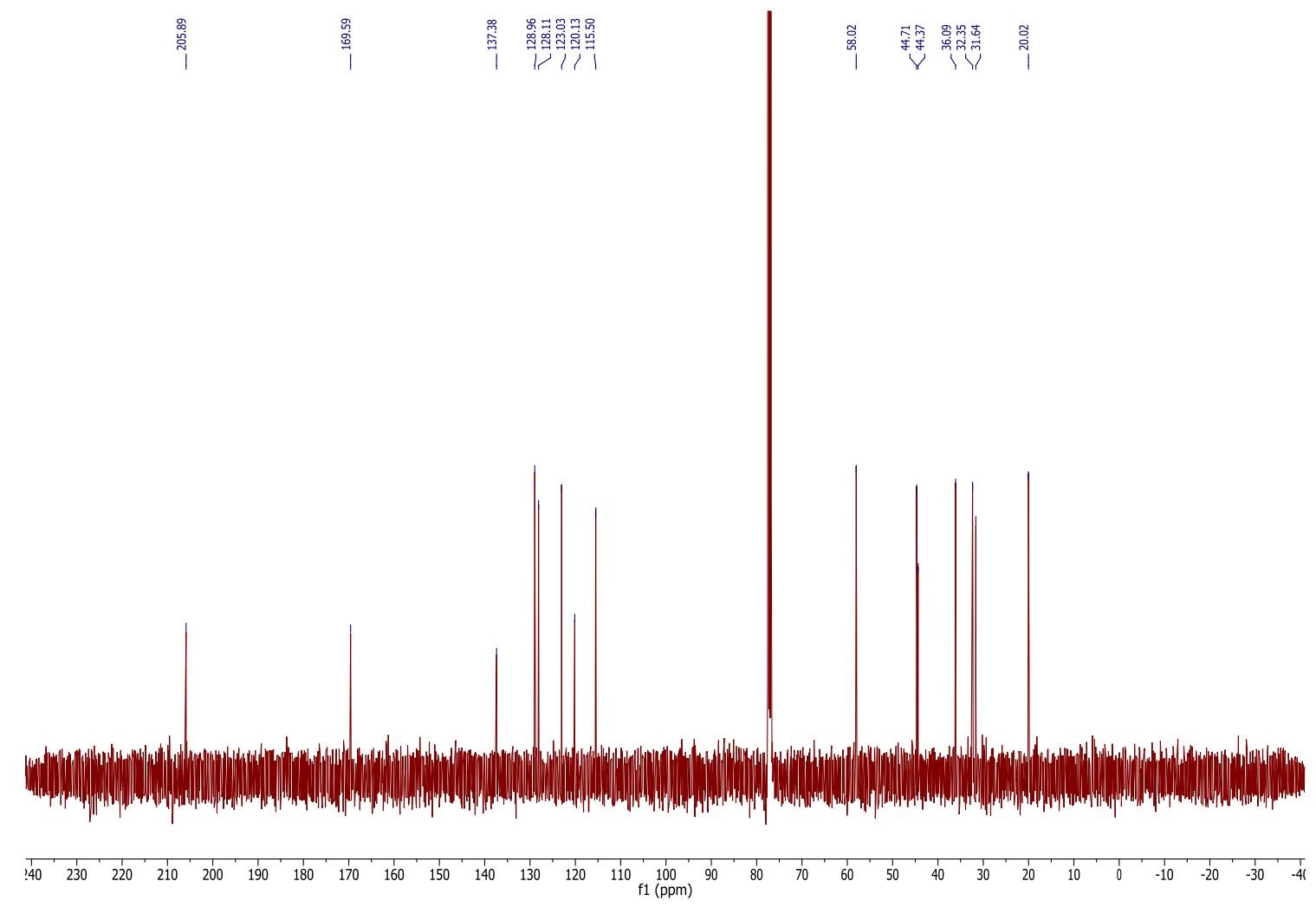



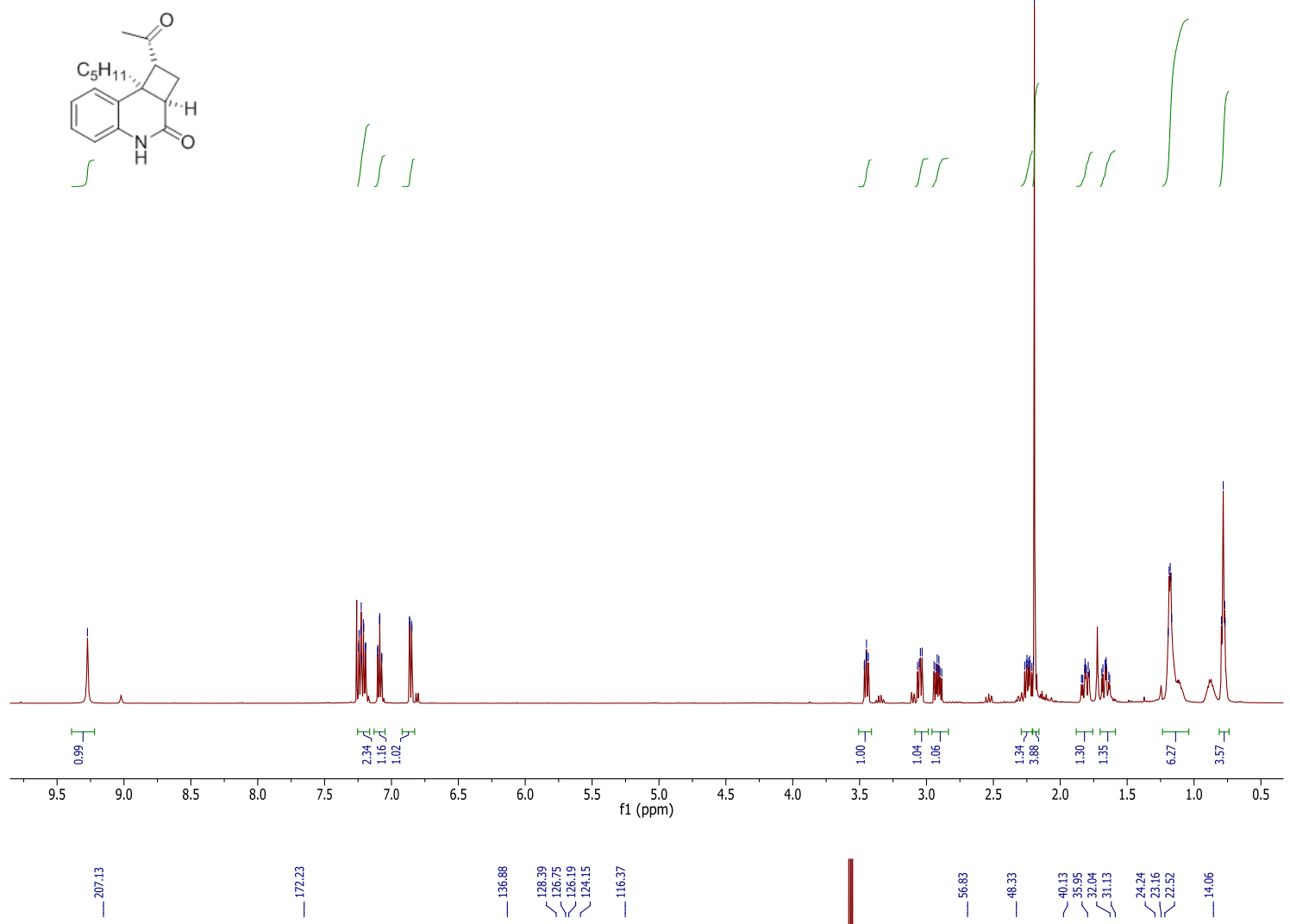

总

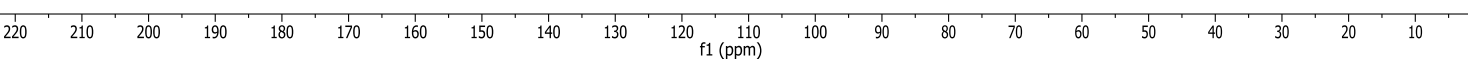



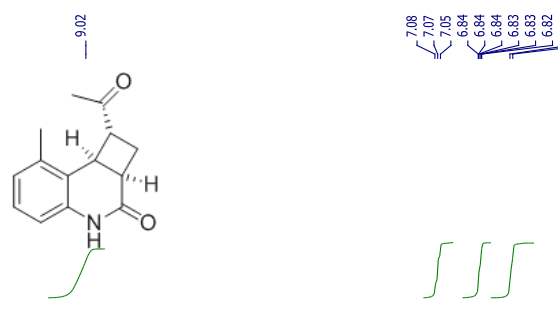

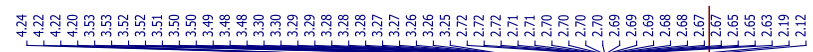
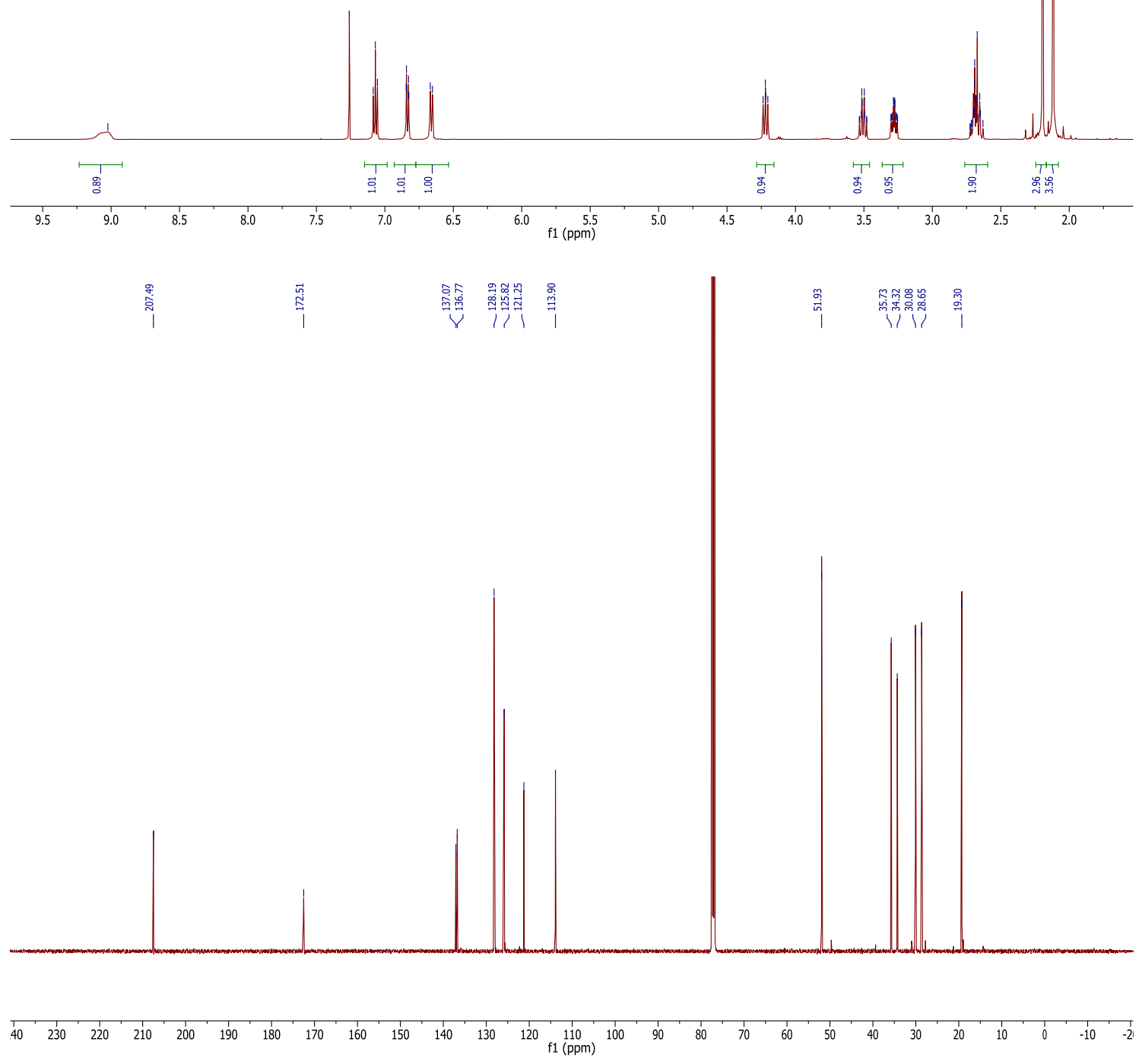

- S63- 


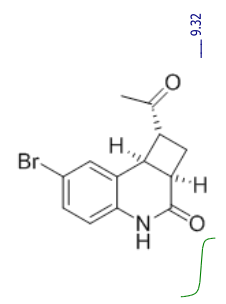

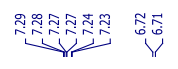

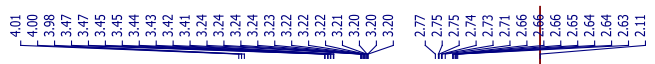

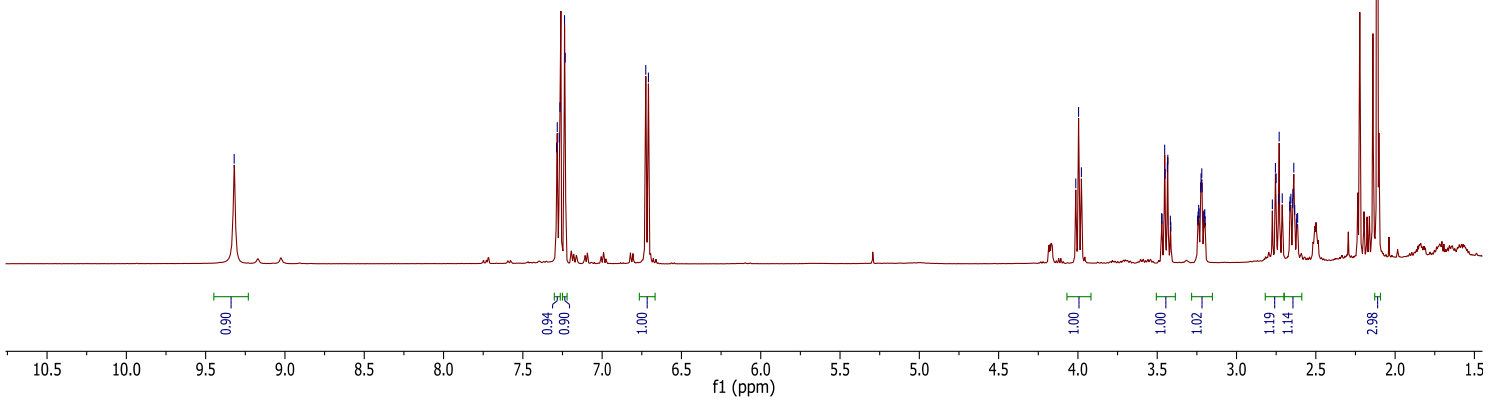

$\vec{\circ}$

总

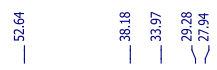
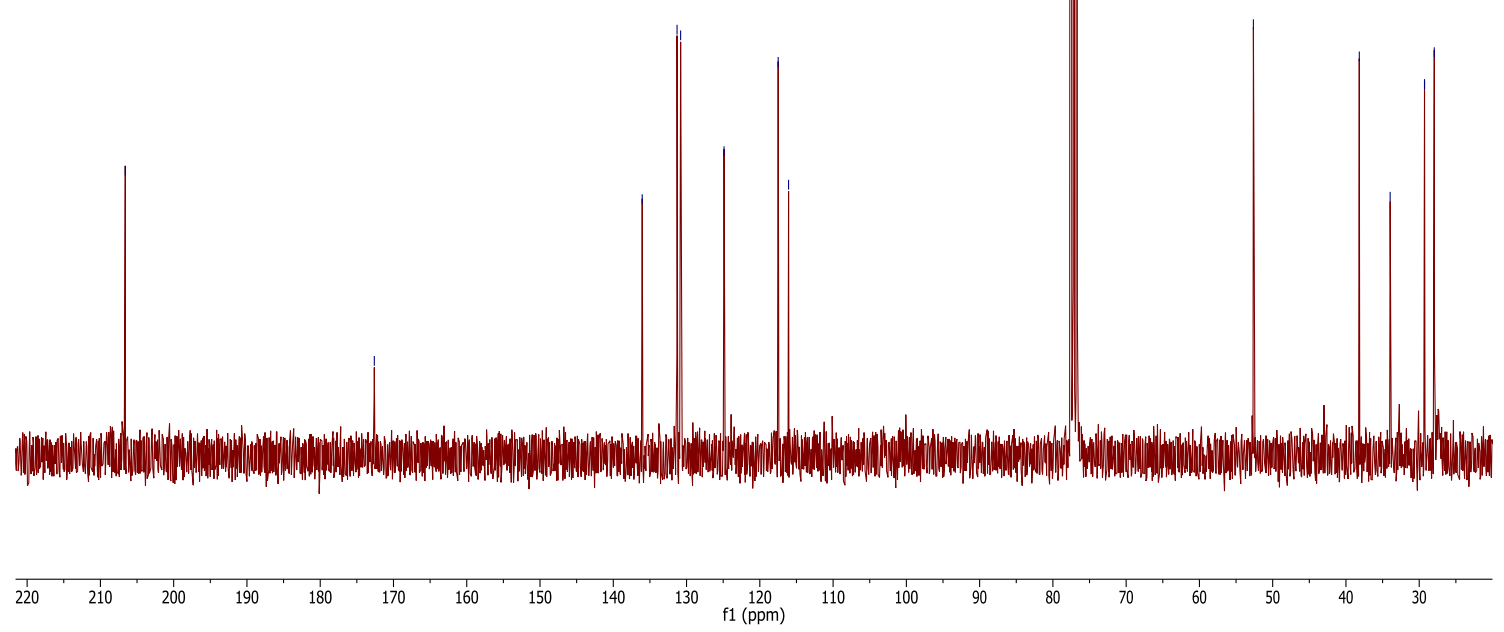

-S64- 


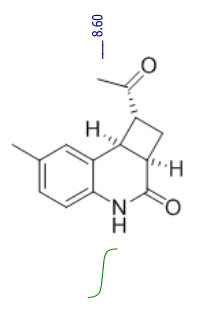

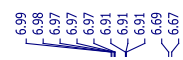

운.
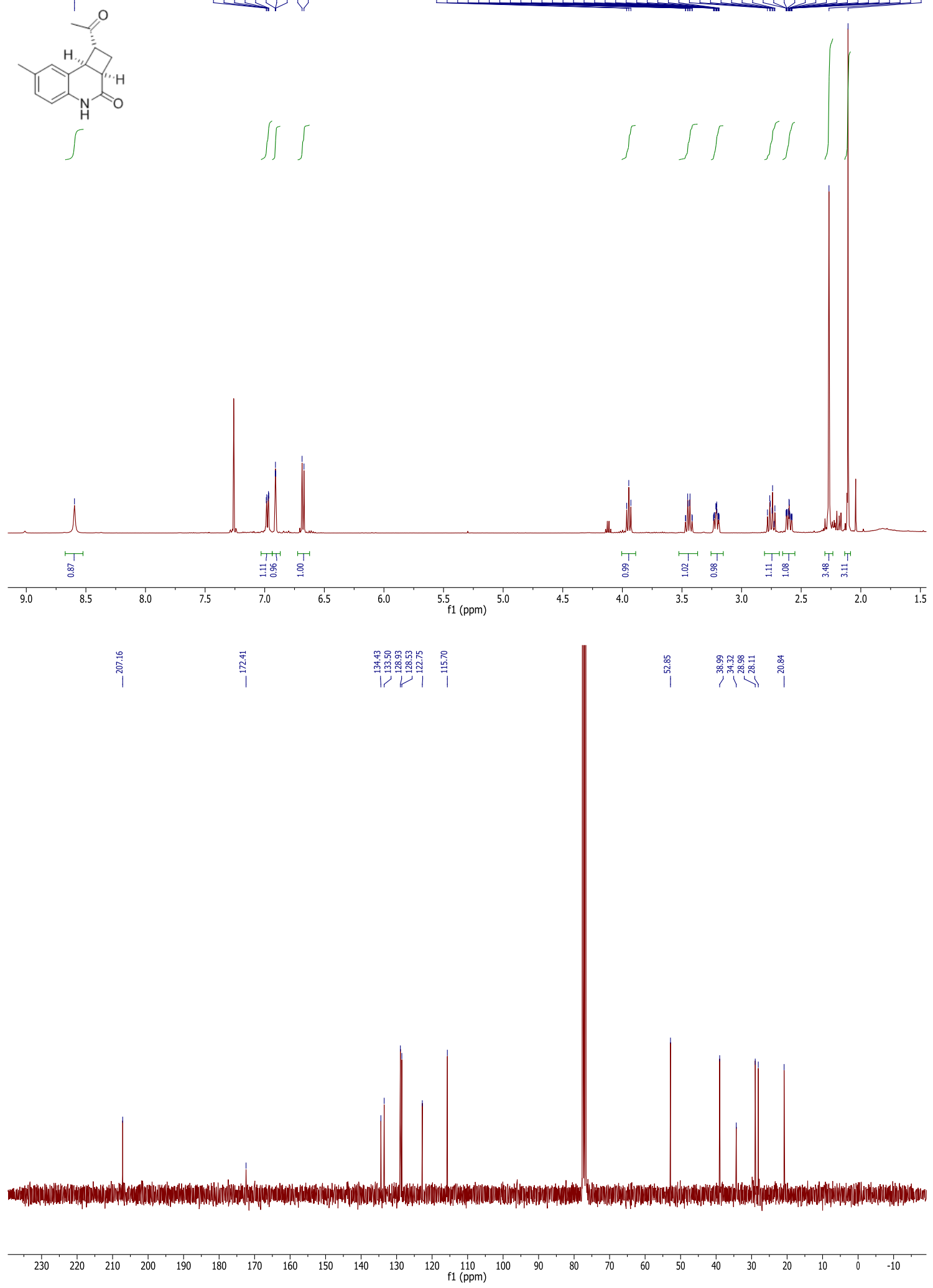

-S65- 


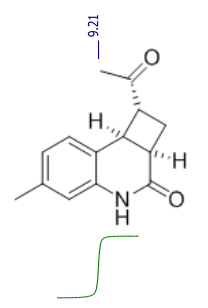

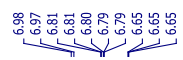

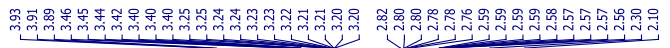

$\iiint$

IJIJ
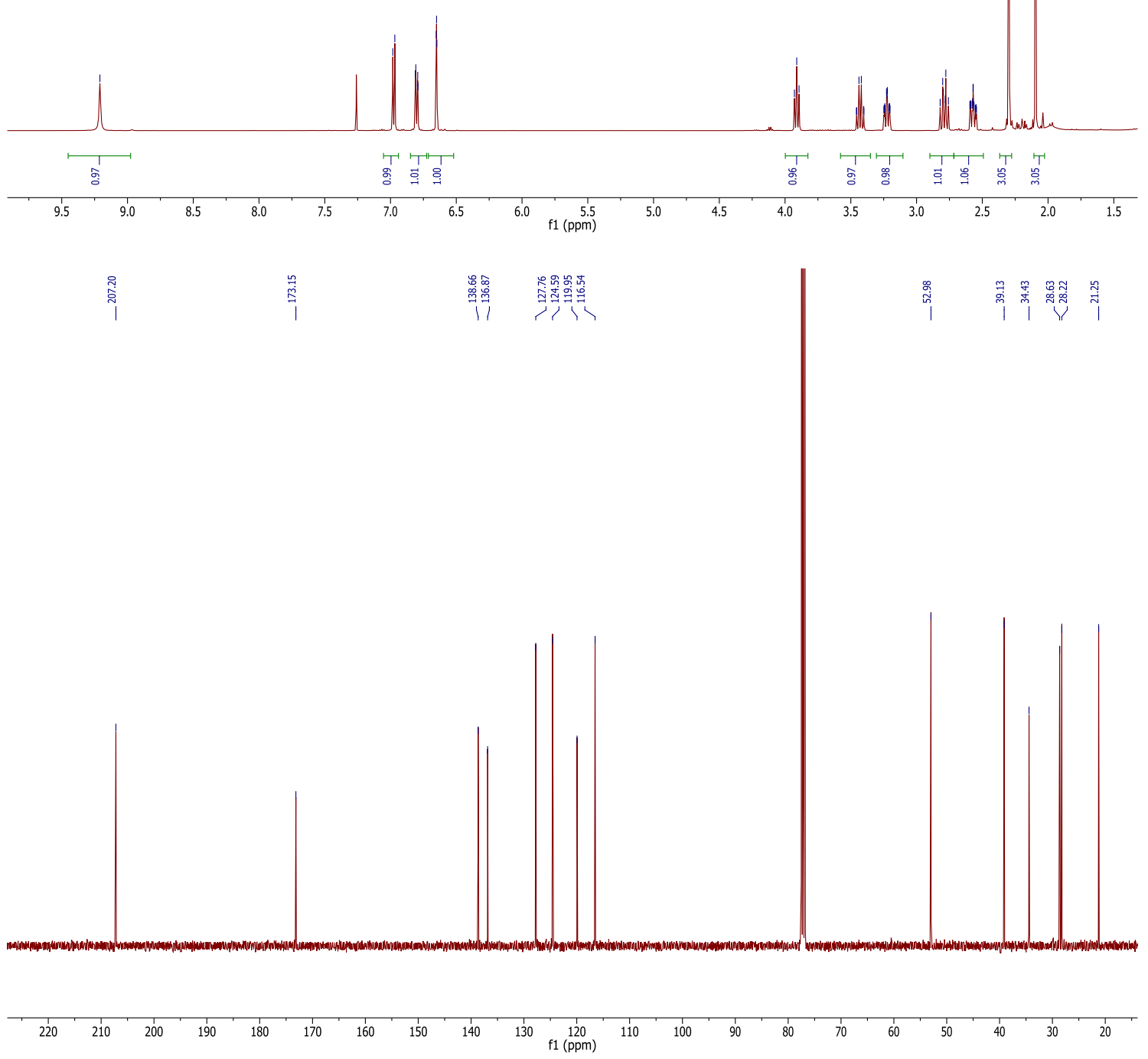

- S66- 

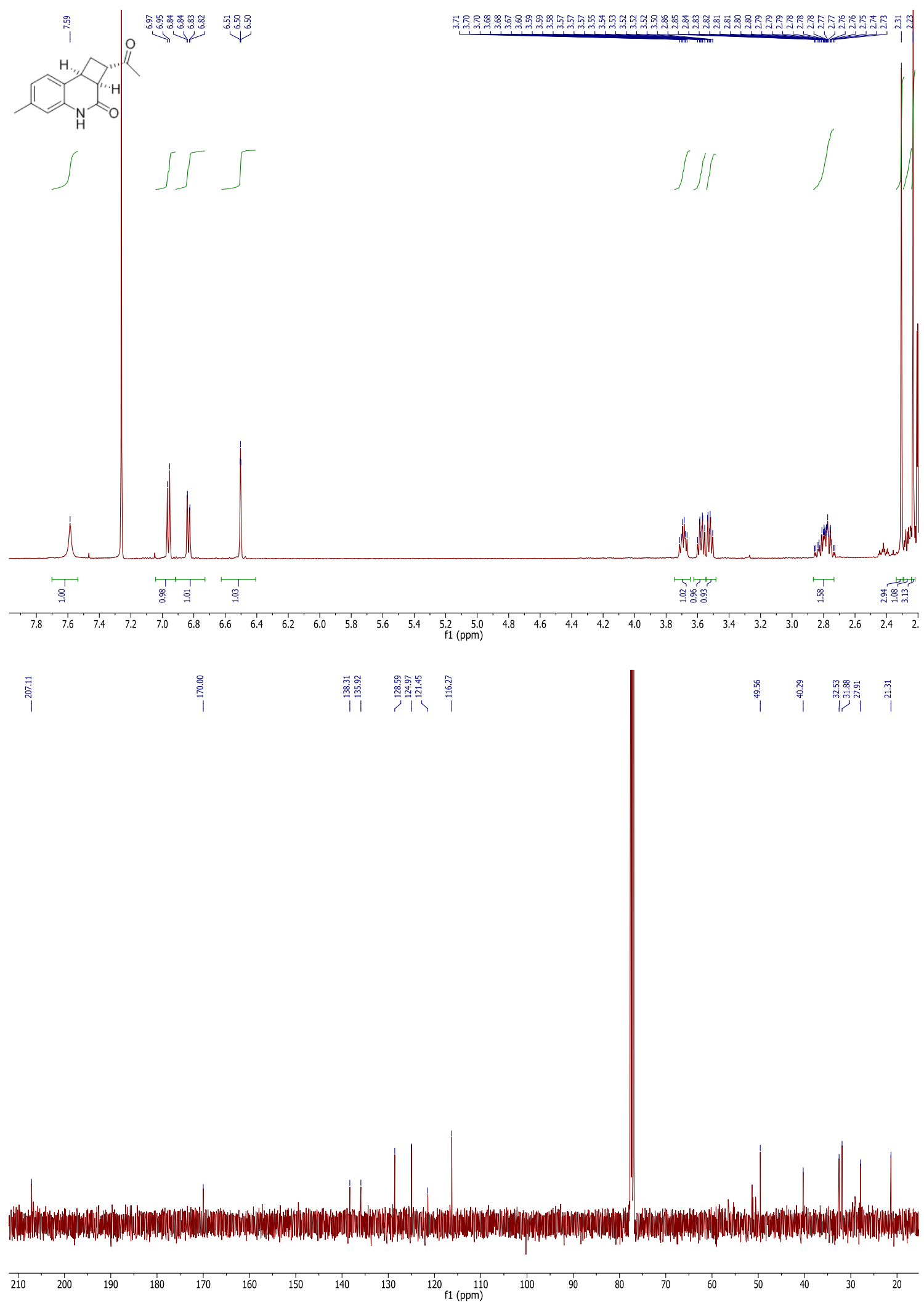


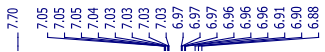

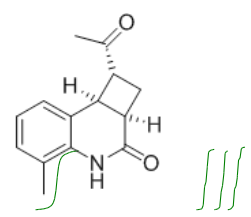

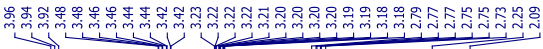

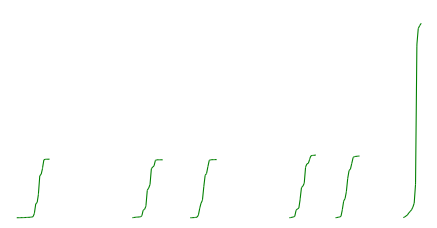

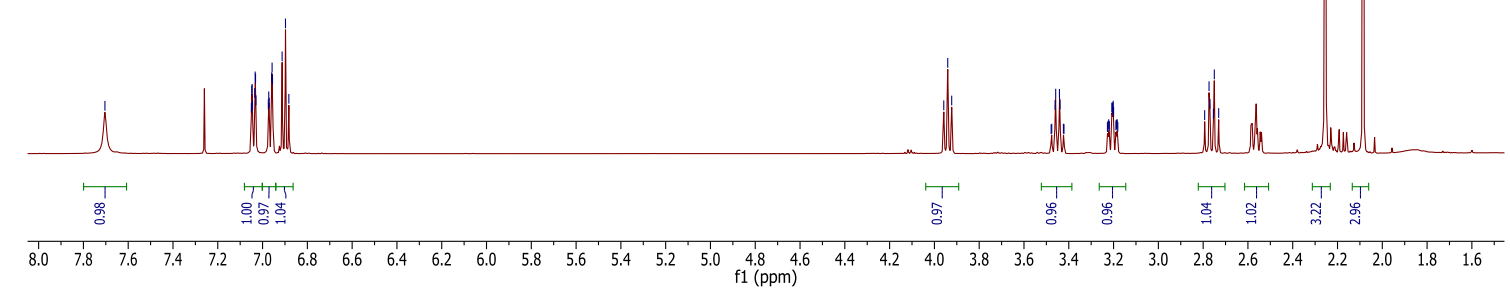

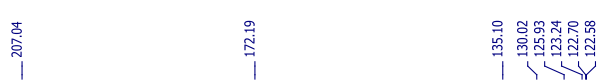

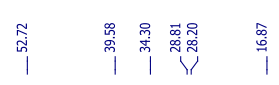

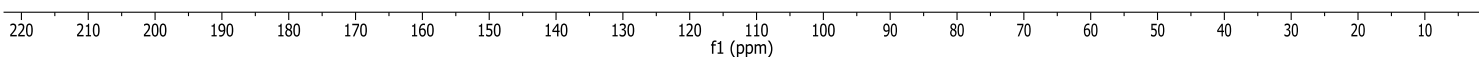



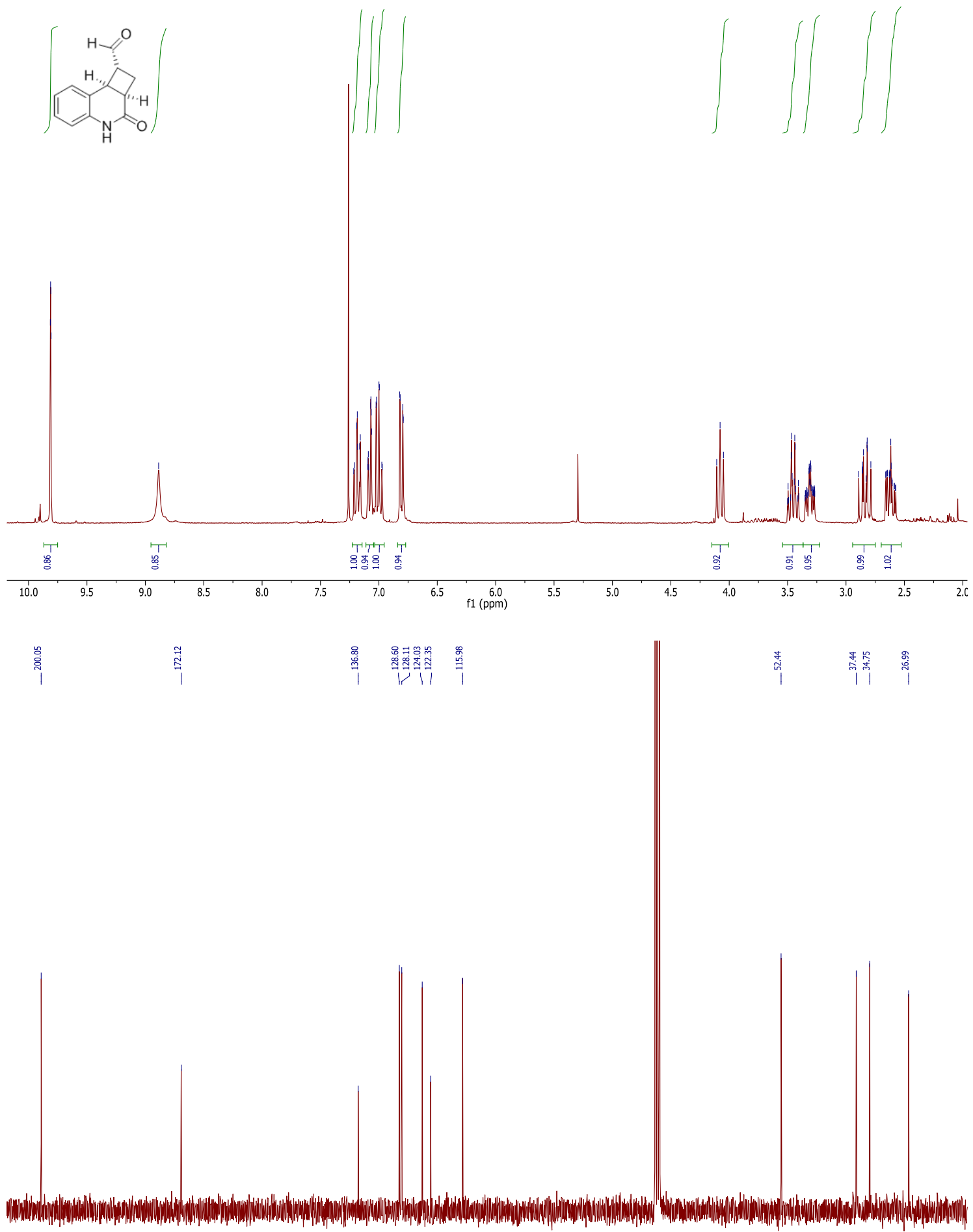

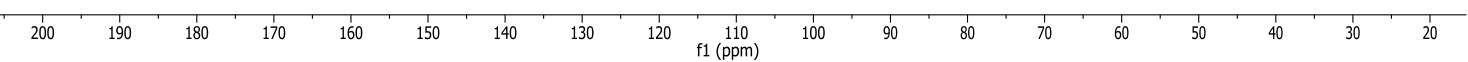



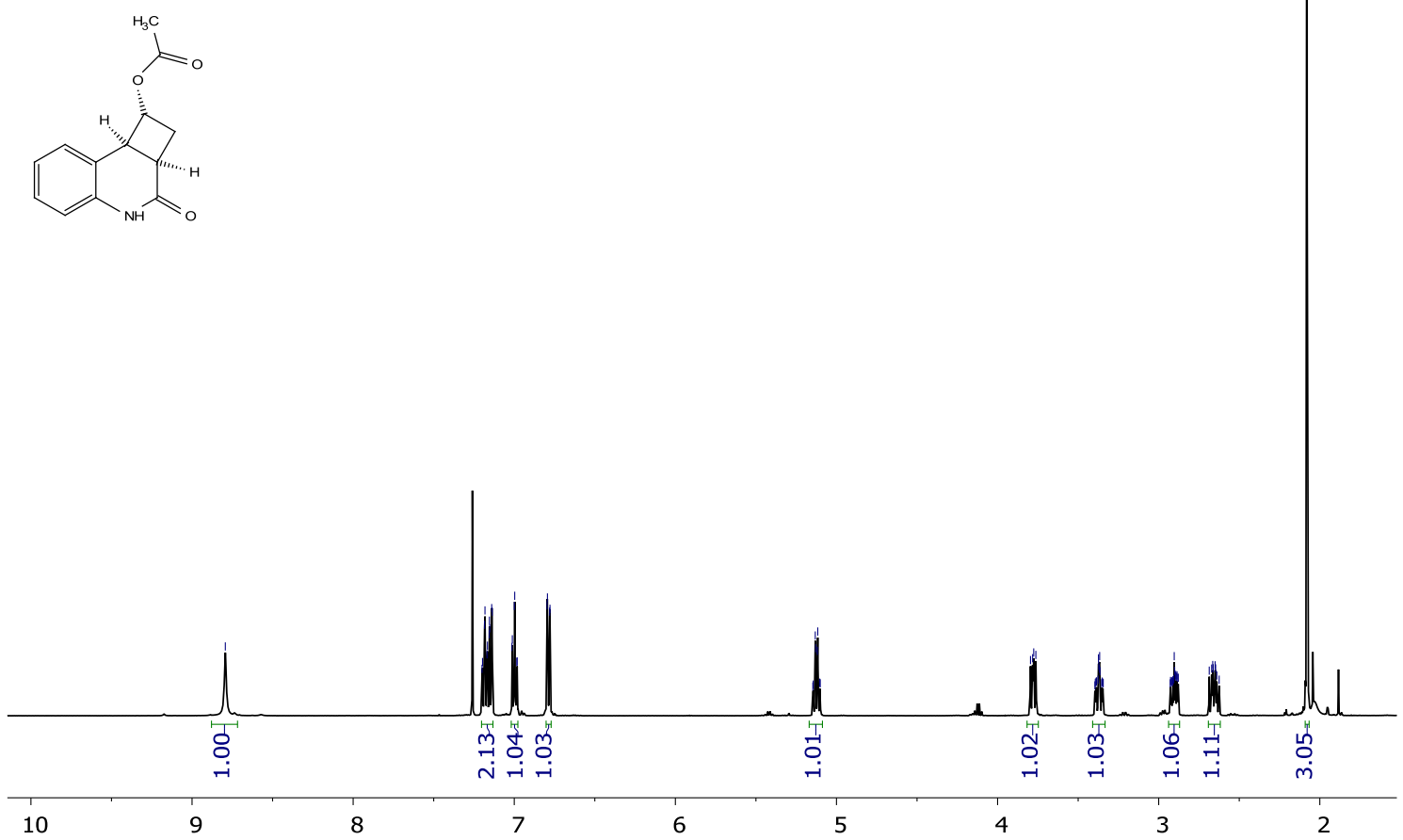

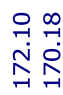

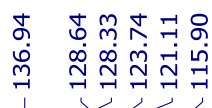

离

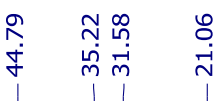

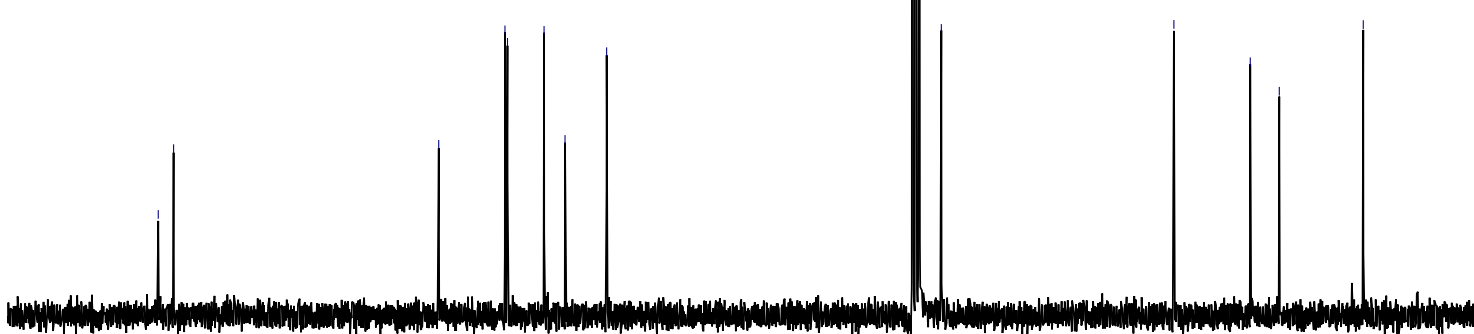

180

160

140

120

100

80

60

40

20 

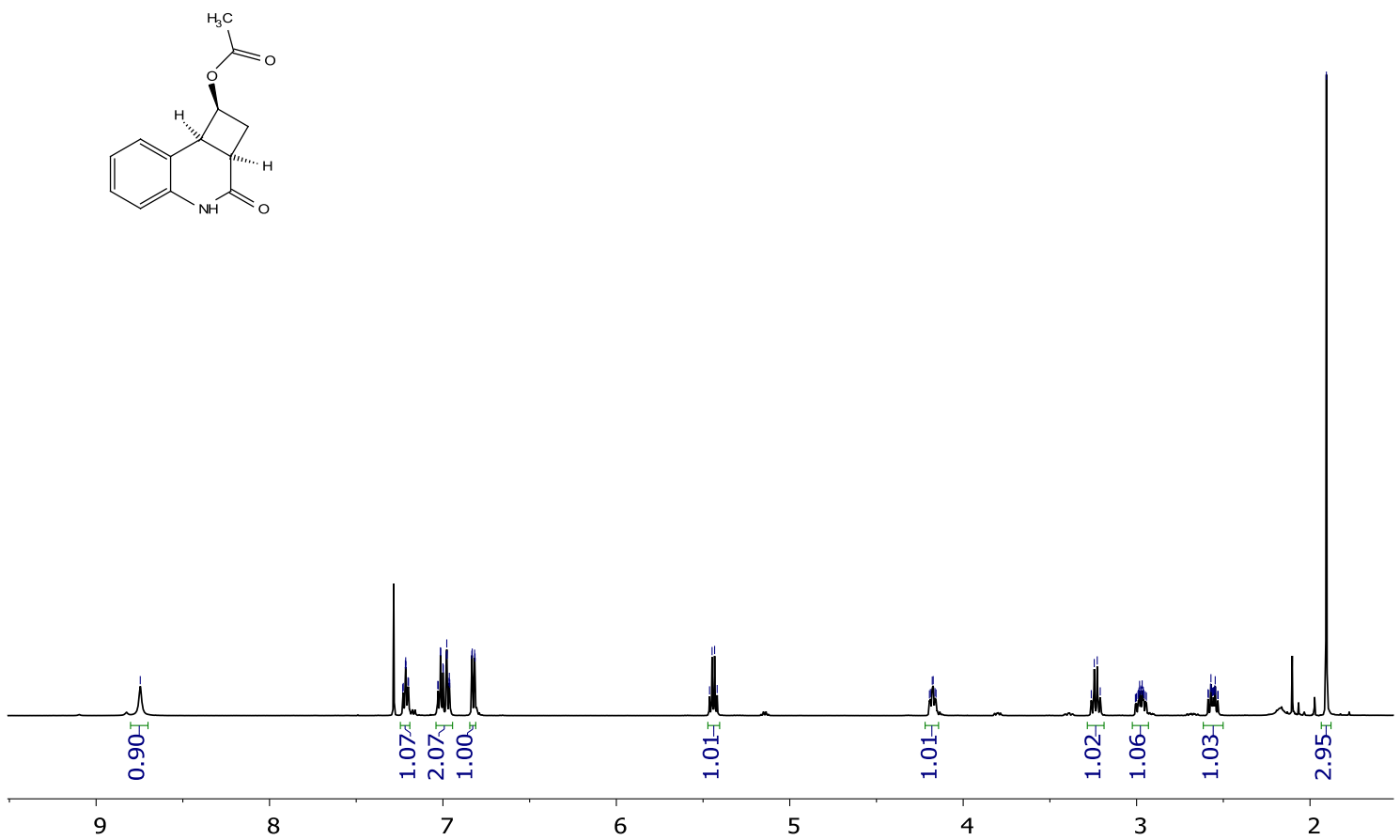

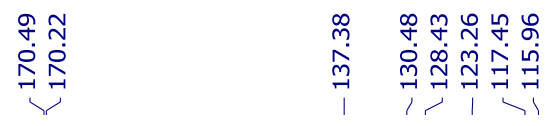

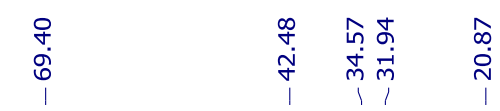

$\begin{array}{llllllllllllllll}170 & 160 & 150 & 140 & 130 & 120 & 110 & 100 & 90 & 80 & 70 & 60 & 50 & 40 & 30 & 20\end{array}$ 


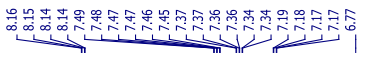

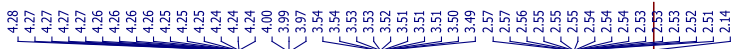

$\overbrace{\mathrm{NH}}^{\mathrm{NH}}$

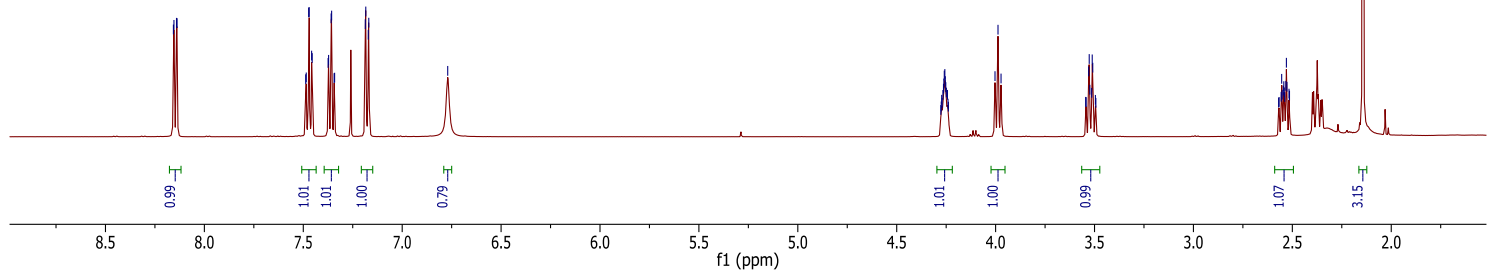

)

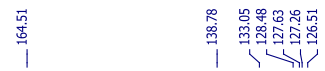

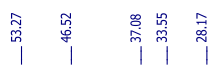

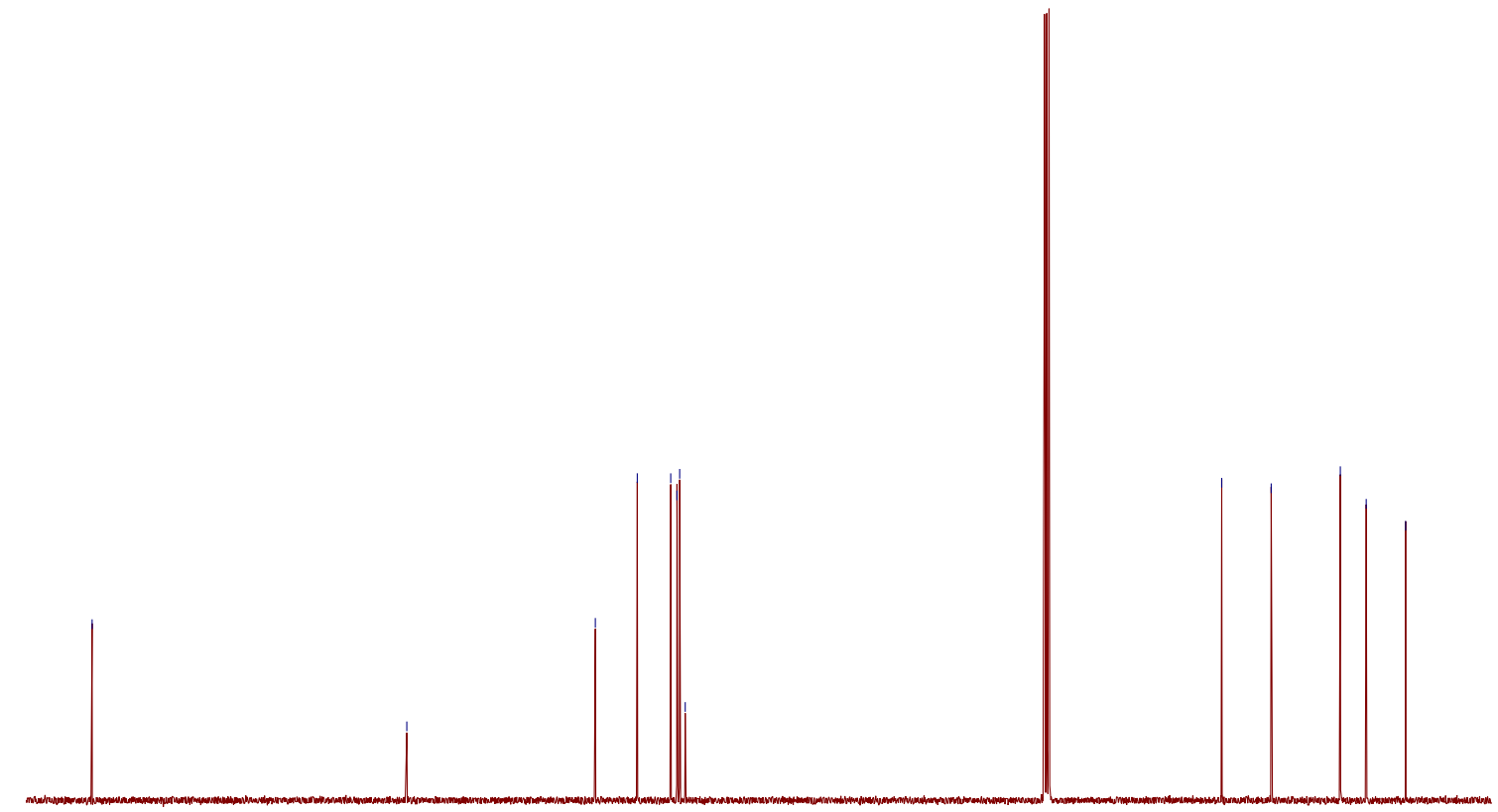

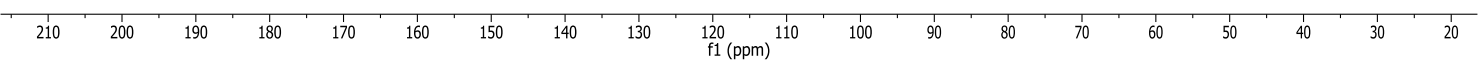



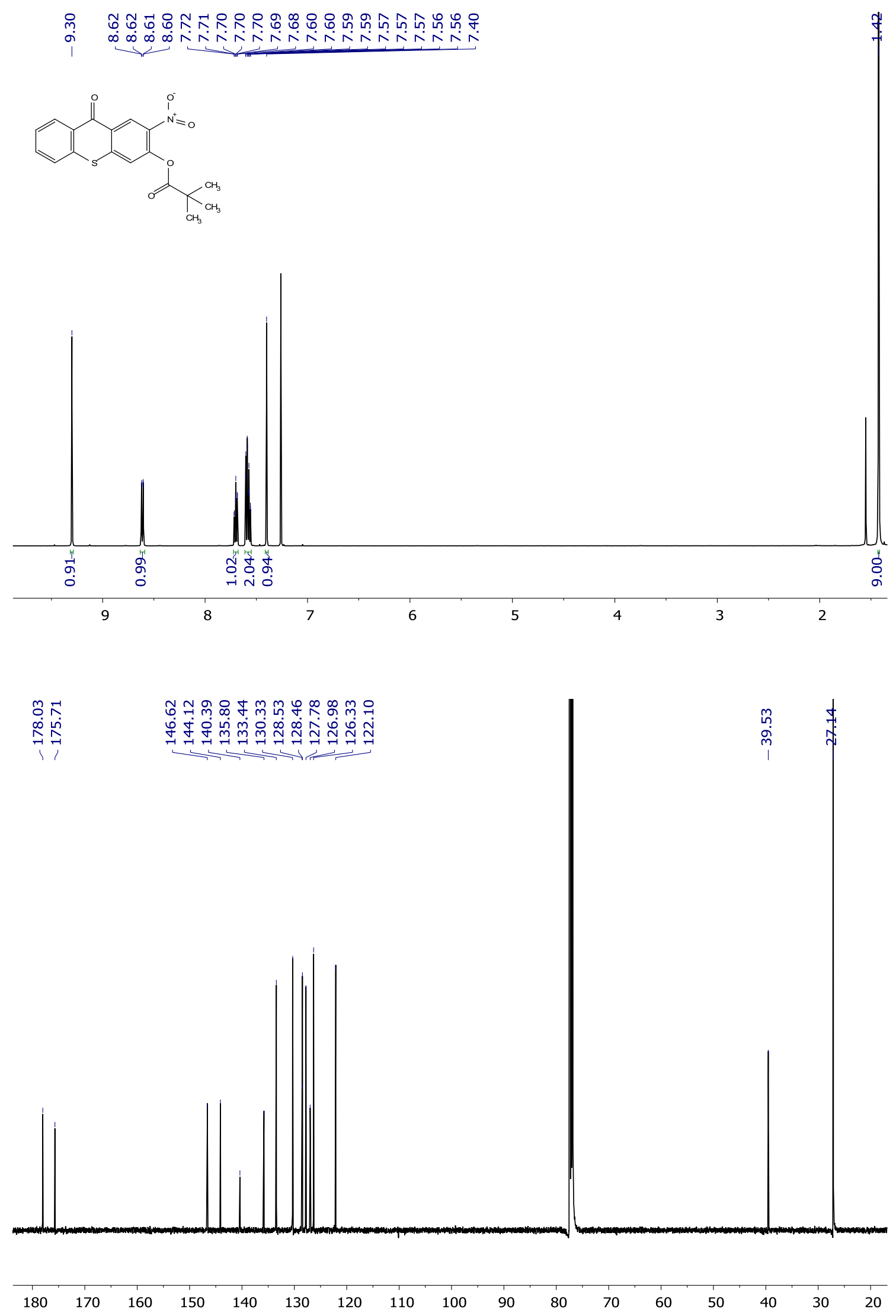


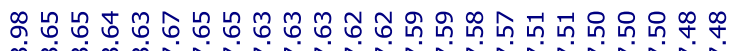

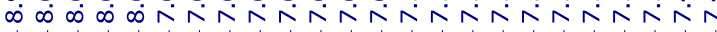
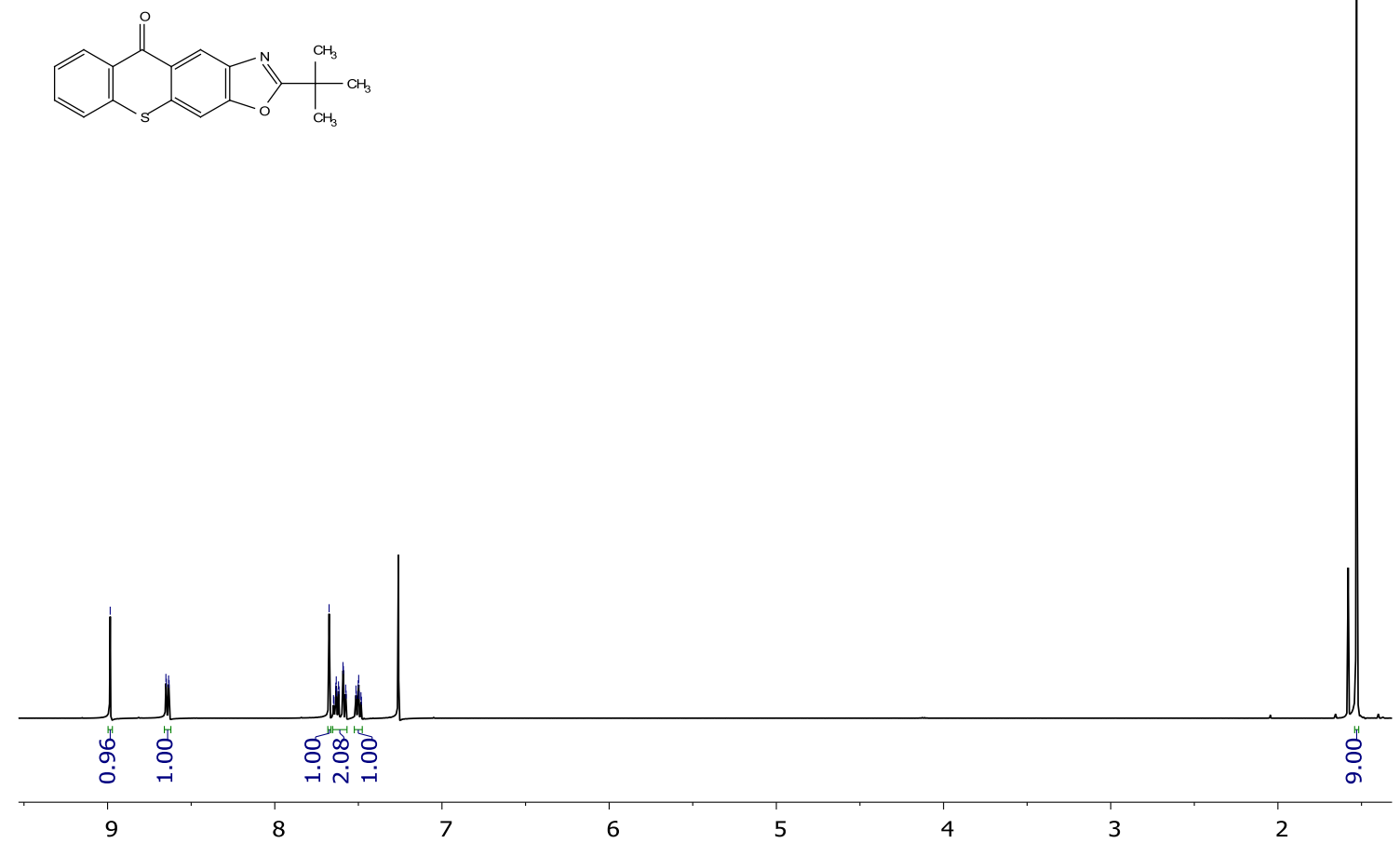

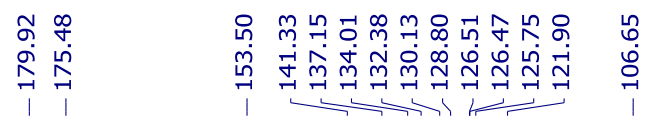

帒先 


\section{Representative HPLC/GC Traces}

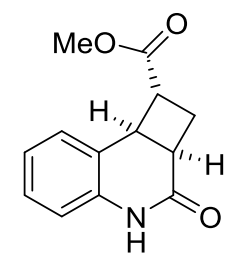

HPLC $($ AD-H, 250×4.6, $n$-hexane/iso-propanol $=90: 10)=\mathbf{4 a}, t_{\mathrm{R}}=15.2 \mathrm{~min}$; ent-4a, $16.8 \mathrm{~min})$.
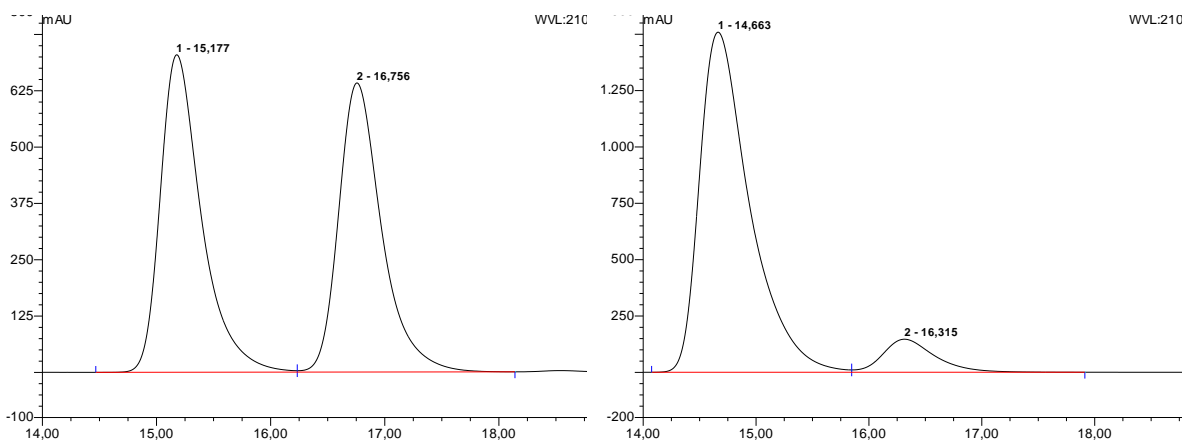

\begin{tabular}{|c|cc|cc|}
\hline & Area & Rel. Area $(\%)$ & Area & Rel.Area $(\%)$ \\
\hline$t_{\mathrm{R}}=15.2 \mathrm{~min}$ & 295.295 & 51.33 & 777.707 & 90.89 \\
\hline$t_{\mathrm{R}}=16.8 \mathrm{~min}$ & 279.945 & 48.67 & 77.978 & 9.11 \\
\hline
\end{tabular}<smiles>CCOC(=O)[C@H]1C[C@H]2C(=O)Nc3ccccc3[C@H]12</smiles>

HPLC (AD-H, 250×4.6, $n$-hexane/iso-propanol $=90: 10)=4 \mathbf{b}, t_{\mathrm{R}}=12.4 \mathrm{~min} ;$ ent-4b, $13.7 \mathrm{~min})$
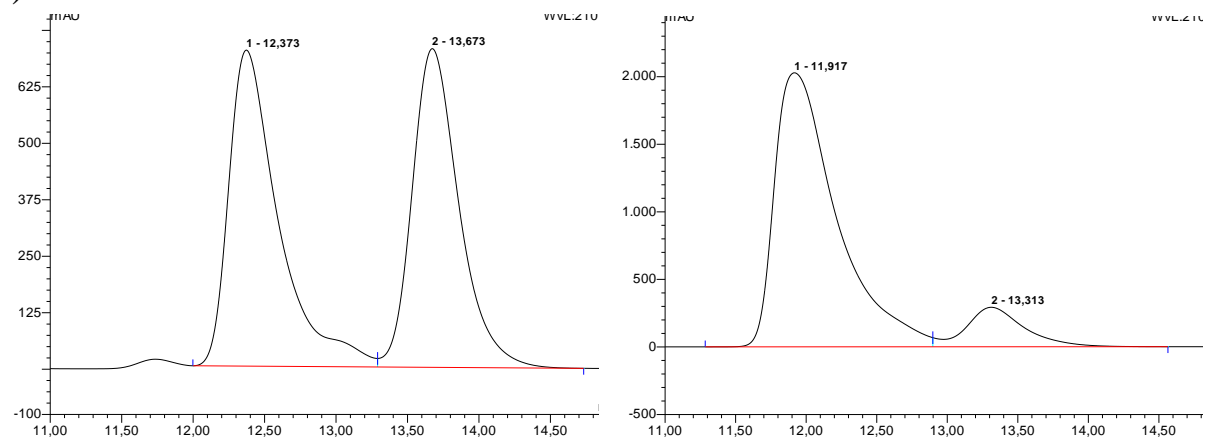

\begin{tabular}{|c|cc|cc|}
\hline & Area & Rel. Area $(\%)$ & Area & Rel. Area $(\%)$ \\
\hline$t_{\mathrm{R}}=12.4 \mathrm{~min}$ & 282.609 & 51.29 & 1030.052 & 88.04 \\
\hline$t_{\mathrm{R}}=13.7 \mathrm{~min}$ & 268.345 & 48.71 & 139.868 & 11.96 \\
\hline
\end{tabular}


HPLC trace of the $0.15 \mathrm{mmol}$ scale reaction of $4 \mathrm{~b}$

HPLC $\left(\right.$ AD-H, 250×4.6, $n$-heptane/iso-propanol = 90:10) $=\mathbf{4 b}, t_{\mathrm{R}}=12.9 \mathrm{~min} ;$ ent-4b, $14.5 \mathrm{~min})$.

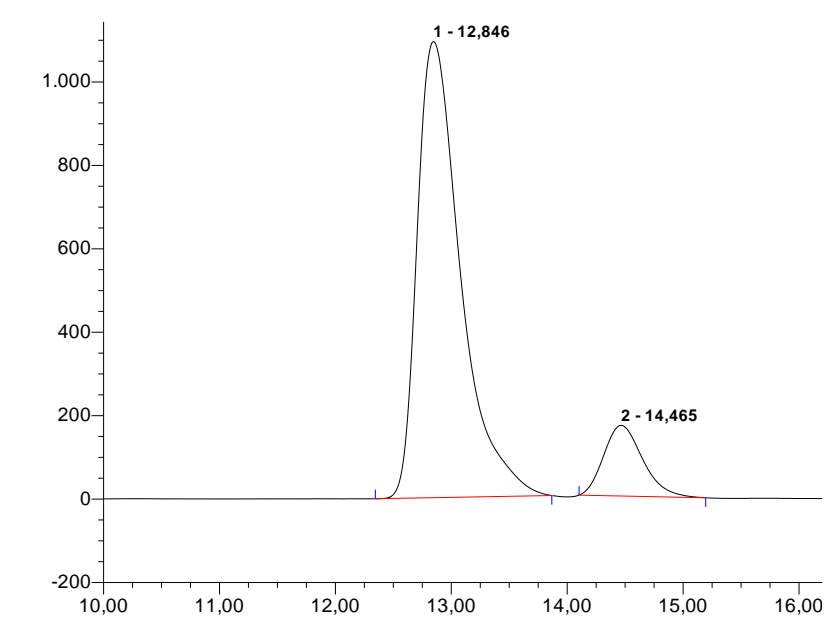

\begin{tabular}{|c|cc|}
\hline & Area & Rel. Area $(\%)$ \\
\hline$t_{\mathrm{R}}=12.9 \mathrm{~min}$ & 1093.542 & 87.79 \\
\hline$t_{\mathrm{R}}=14.5 \mathrm{~min}$ & 159.299 & 12.21 \\
\hline
\end{tabular}

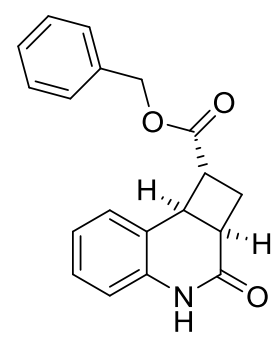

HPLC $(\mathrm{AD}-\mathrm{H}, 250 \times 4.6, n$-hexane/iso-propanol $=90: 10)=\mathbf{4 c}, t_{\mathrm{R}}=18.1 \mathrm{~min} ;$ ent-4c, $24.2 \mathrm{~min})$.
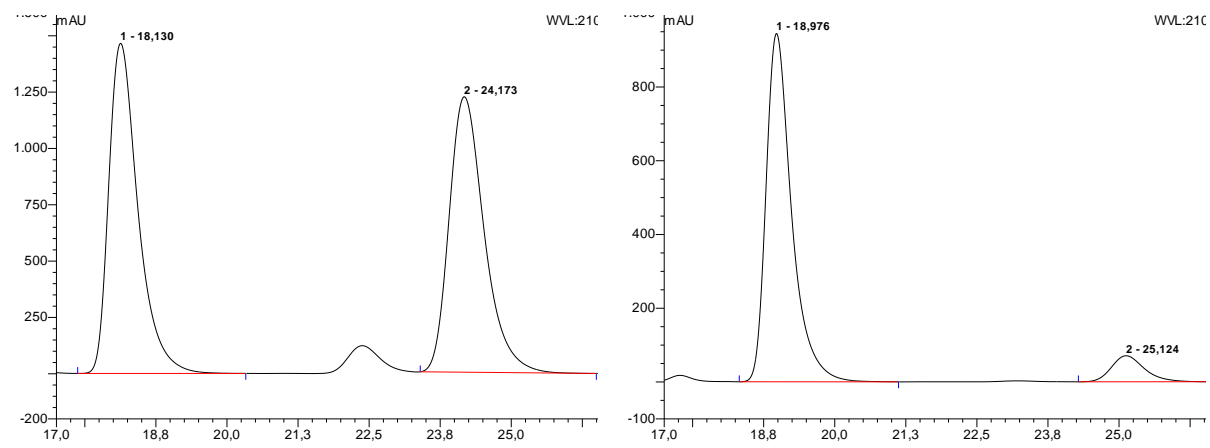

\begin{tabular}{|c|cc|cc|}
\hline & Area & Rel. Area $(\%)$ & Area & Rel. Area $(\%)$ \\
\hline$t_{\mathrm{R}}=18.1 \mathrm{~min}$ & 874.215 & 50.01 & 492.605 & 91.21 \\
\hline$t_{\mathrm{R}}=24.2 \mathrm{~min}$ & 873.870 & 49.99 & 47.473 & 8.79 \\
\hline
\end{tabular}




\section{HPLC trace of the $0.15 \mathrm{mmol}$ scale reaction of $4 \mathrm{c}$}

HPLC $($ AD-H, 250×4.6, $n$-heptane/iso-propanol $=90: 10)=4 \mathbf{c}, t_{\mathrm{R}}=18.6 \mathrm{~min} ;$ ent-4c, $25.4 \mathrm{~min})$.

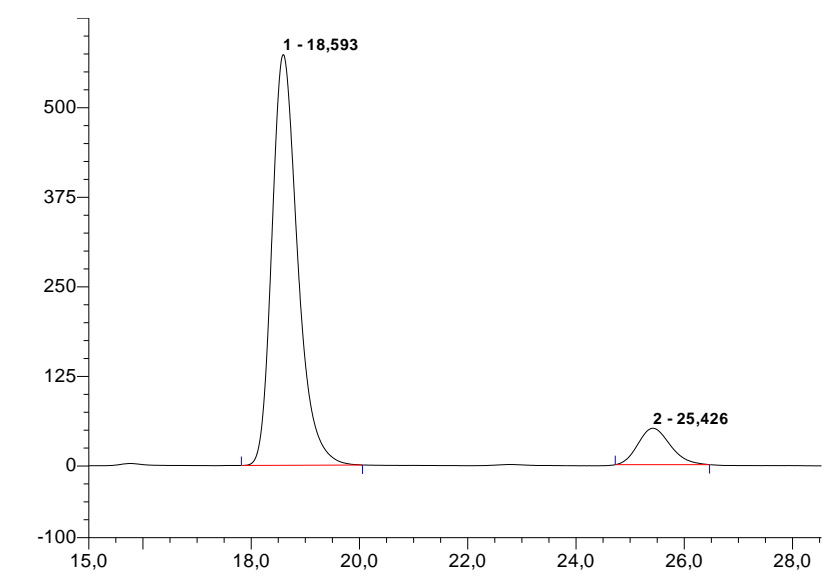

\begin{tabular}{|c|cc|}
\hline & Area & Rel. Area $(\%)$ \\
\hline$t_{\mathrm{R}}=18.6 \mathrm{~min}$ & 573.215 & 89.99 \\
\hline$t_{\mathrm{R}}=25 . \mathrm{min}$ & 50.792 & 10.01 \\
\hline
\end{tabular}

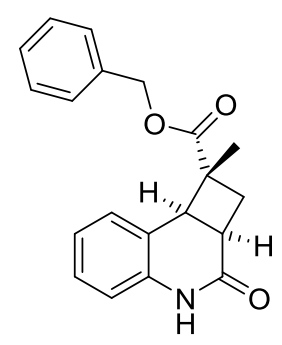

HPLC $($ AD-H, 250×4.6, $n$-hexane/iso-propanol $=90: 10)=\mathbf{4 d}, t_{\mathrm{R}}=14.4 \mathrm{~min}$; ent-4d, $19.6 \mathrm{~min})$.
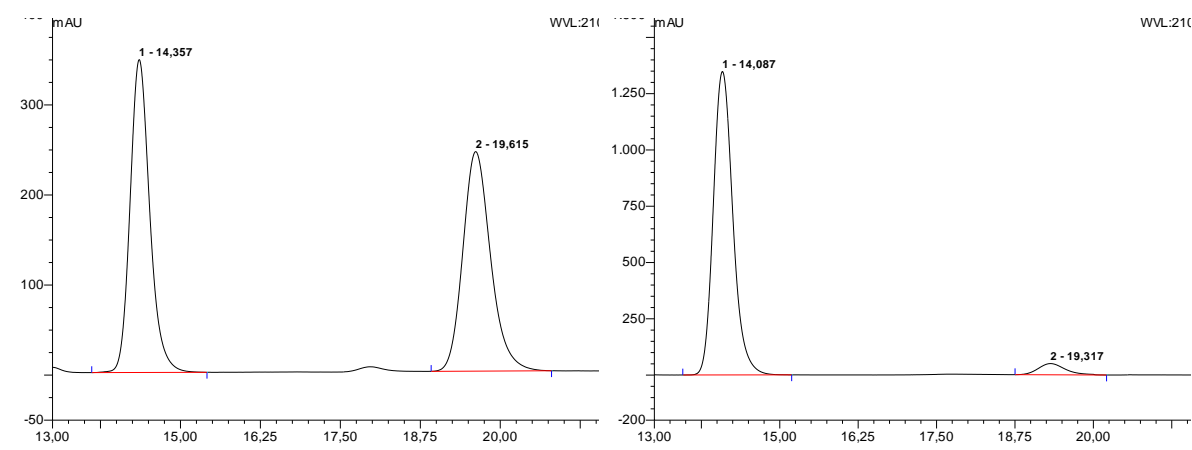

\begin{tabular}{|c|cc|cc|}
\hline & Area & Rel. Area $(\%)$ & Area & Rel. Area $(\%)$ \\
\hline$t_{\mathrm{R}}=14.4 \mathrm{~min}$ & 124.001 & 50.26 & 242.833 & 92.41 \\
\hline$t_{\mathrm{R}}=19.6 \mathrm{~min}$ & 122.735 & 49.74 & 19.931 & 7.59 \\
\hline
\end{tabular}


HPLC trace of the $0.15 \mathrm{mmol}$ scale reaction of $4 \mathrm{~d}$

HPLC $(A D-H, 250 \times 4.6, n$-heptane/iso-propanol $=90: 10)=\mathbf{4 d}, t_{\mathrm{R}}=13.0 \mathrm{~min} ;$ ent-4d, $18.9 \mathrm{~min})$.

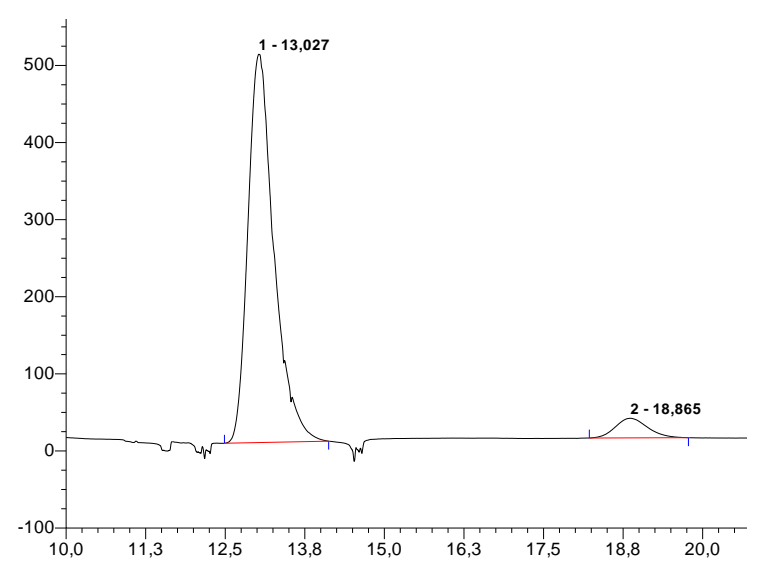

\begin{tabular}{|c|cc|}
\hline & Area & Rel. Area $(\%)$ \\
\hline$t_{\mathrm{R}}=13.0 \mathrm{~min}$ & 238.152 & 94.11 \\
\hline$t_{\mathrm{R}}=18.9 \mathrm{~min}$ & 14.908 & 5.89 \\
\hline
\end{tabular}

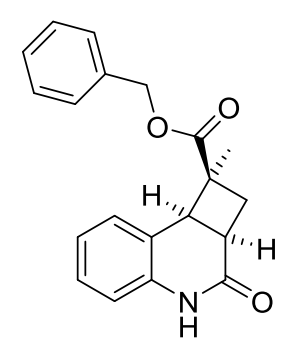

HPLC (AD-H, 250 $\times 4.6, n$-hexane/iso-propanol $=80: 20)=\mathbf{S 1 3}, t_{\mathrm{R}}=13.0 \mathrm{~min} ;$ ent-S13, $22.8 \mathrm{~min})$.
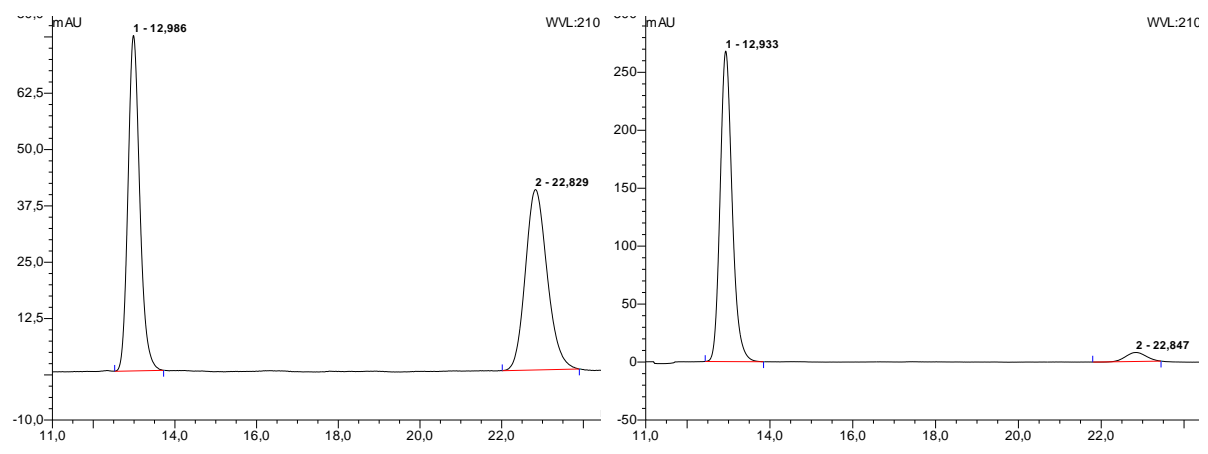

\begin{tabular}{|c|cc|cc|}
\hline & Area & Rel. Area $(\%)$ & Area & Rel. Area $(\%)$ \\
\hline$t_{\mathrm{R}}=13.0 \mathrm{~min}$ & 24.877 & 50.14 & 90.037 & 95.36 \\
\hline$t_{\mathrm{R}}=22.8 \mathrm{~min}$ & 24.743 & 49.86 & 4.377 & 4.64 \\
\hline
\end{tabular}


HPLC trace of the $0.15 \mathrm{mmol}$ scale reaction of S13

HPLC $($ AD-H, 250×4.6, $n$-heptane/iso-propanol $=80: 20)=\mathbf{S 1 3}, t_{\mathrm{R}}=12.8 \mathrm{~min}$; entS13, $22.5 \mathrm{~min})$.

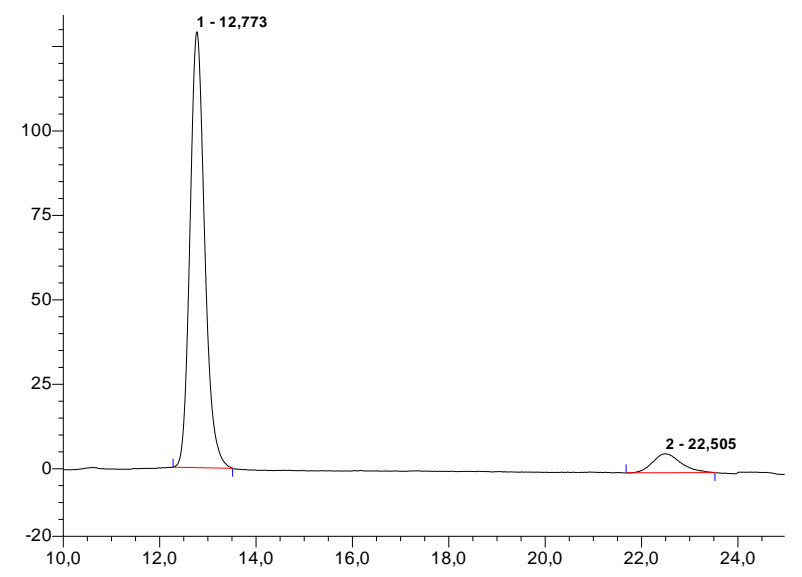

\begin{tabular}{|c|cc|}
\hline & Area & Rel. Area $(\%)$ \\
\hline$t_{\mathrm{R}}=12.8 \mathrm{~min}$ & 45.652 & 92.28 \\
\hline$t_{\mathrm{R}}=22.5 \mathrm{~min}$ & 3.818 & 7.72 \\
\hline
\end{tabular}

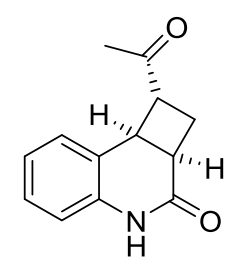

HPLC $(\mathrm{OJ}-\mathrm{H}, 250 \times 4.6, n$-hexane/iso-propanol $=80: 20)=e n t-4 \mathbf{e}, t_{\mathrm{R}}=17.5 \mathrm{~min} ; \mathbf{4 e}$, $20.9 \mathrm{~min})$.
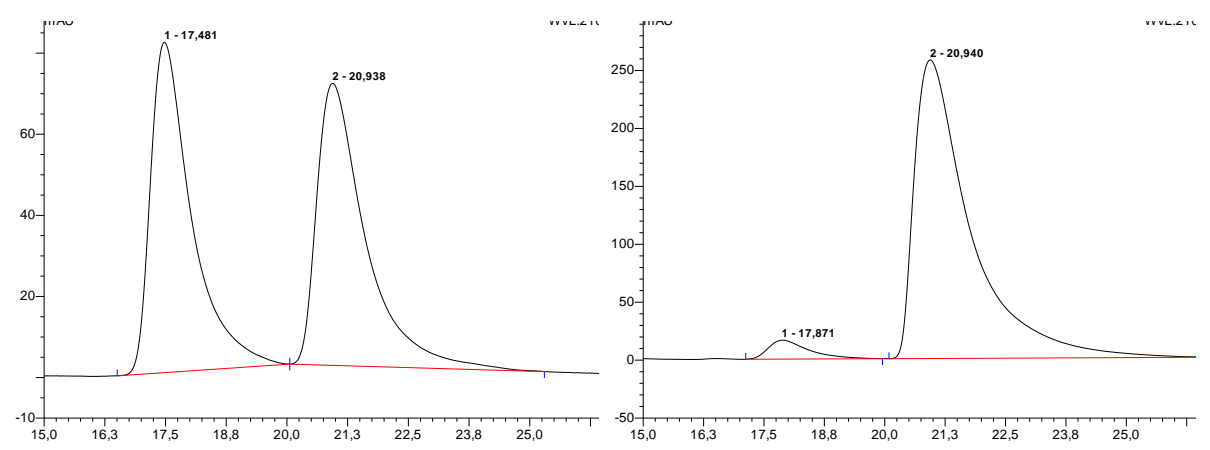

\begin{tabular}{|c|cc|cc|}
\hline & Area & Rel. Area $(\%)$ & Area & Rel. Area $(\%)$ \\
\hline$t_{\mathrm{R}}=17.5 \mathrm{~min}$ & 80.080 & 49.78 & 15.823 & 4.36 \\
\hline$t_{\mathrm{R}}=20.9 \mathrm{~min}$ & 80.803 & 50.22 & 347.064 & 95.64 \\
\hline
\end{tabular}




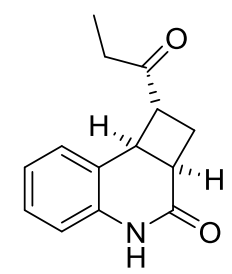

HPLC (AD-H, 250×4.6, $n$-hexane/iso-propanol = 90:10) $=\mathbf{4 f}, t_{\mathrm{R}}=15.4 \mathrm{~min}$; ent-4f, $16.9 \mathrm{~min})$.
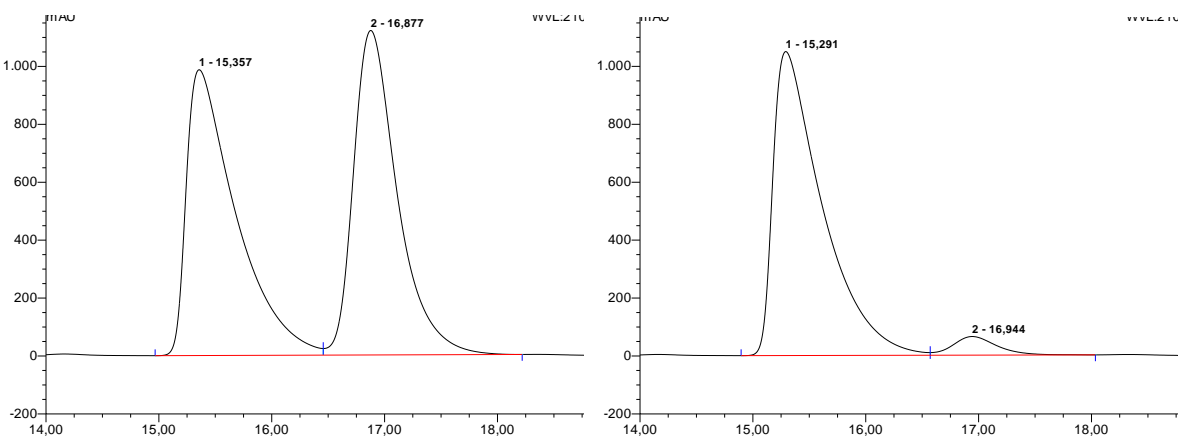

\begin{tabular}{|c|cc|cc|}
\hline & Area & Rel. Area $(\%)$ & Area & Rel. Area $(\%)$ \\
\hline$t_{\mathrm{R}}=15.4 \mathrm{~min}$ & 501.803 & 49.81 & 530.416 & 94.79 \\
\hline$t_{\mathrm{R}}=16.9 \mathrm{~min}$ & 505.639 & 50.19 & 29.149 & 5.21 \\
\hline
\end{tabular}

HPLC trace of the $0.15 \mathrm{mmol}$ scale reaction of $4 \mathrm{f}$

HPLC $(\mathrm{AD}-\mathrm{H}, 250 \times 4.6, n$-heptane/iso-propanol $=90: 10)=\mathbf{4 f}, t_{\mathrm{R}}=15.9 \min ;$ ent-4f, $17.3 \mathrm{~min})$.

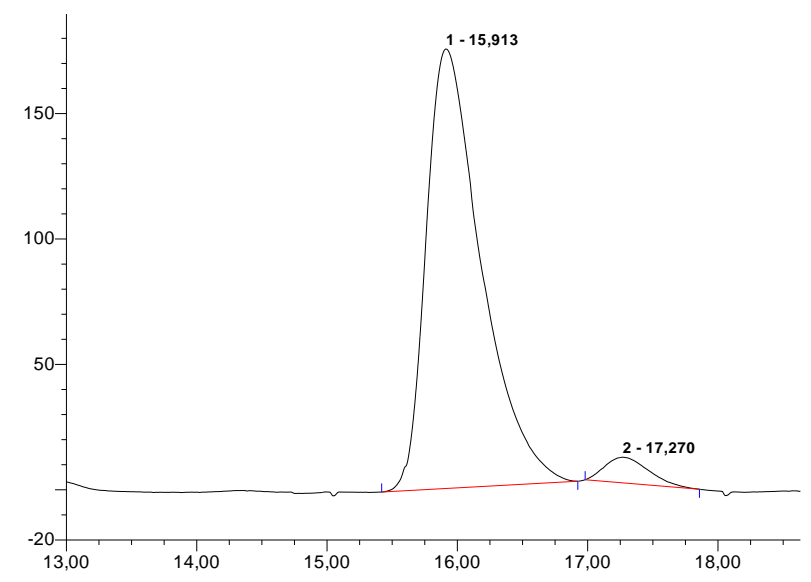

\begin{tabular}{|c|cc|}
\hline & Area & Rel. Area $(\%)$ \\
\hline$t_{\mathrm{R}}=15.9 \mathrm{~min}$ & 85.937 & 95.55 \\
\hline$t_{\mathrm{R}}=17.3 \mathrm{~min}$ & 4.003 & 4.45 \\
\hline
\end{tabular}




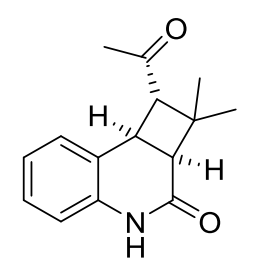

HPLC (AD-H, 250×4.6, $n$-hexane/iso-propanol = 90:10) $=$ ent-4g, $t_{\mathrm{R}}=16.7 \mathrm{~min} ; \mathbf{4 g}$, $30.1 \mathrm{~min}$ ).
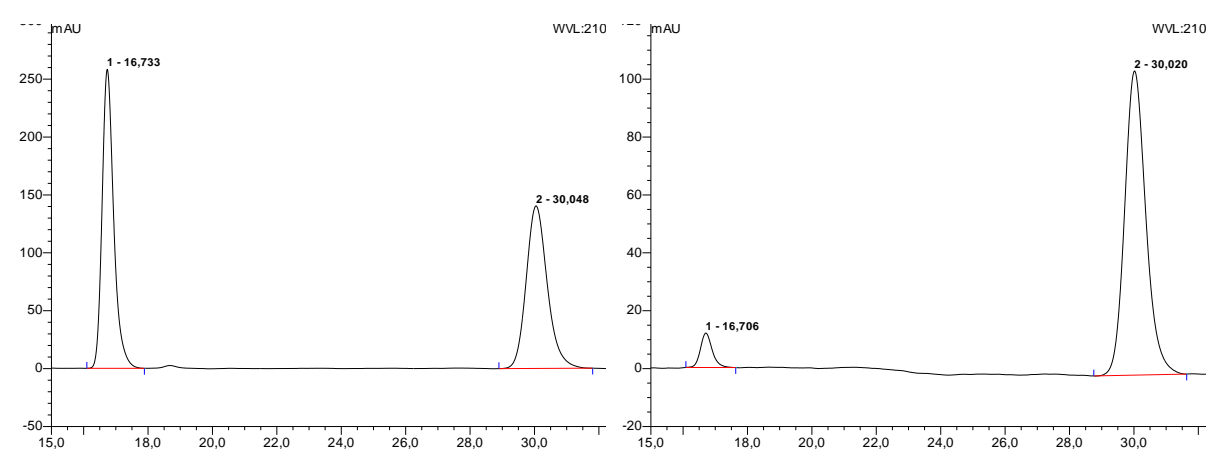

\begin{tabular}{|c|cc|cc|}
\hline & Area & Rel. Area $(\%)$ & Area & Rel. Area $(\%)$ \\
\hline$t_{\mathrm{R}}=16.7 \mathrm{~min}$ & 107.983 & 50.35 & 5.046 & 5.92 \\
\hline$t_{\mathrm{R}}=30.1 \mathrm{~min}$ & 106.463 & 49.65 & 80.256 & 94.08 \\
\hline
\end{tabular}

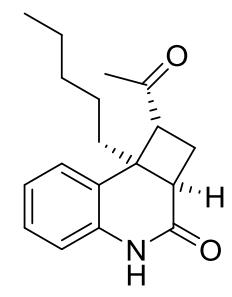

HPLC $\left(\mathrm{OJ}-\mathrm{H}, 250 \times 4.6, n\right.$-hexane/iso-propanol = 95:5) $=$ ent-4h, $t_{\mathrm{R}}=22.5 \mathrm{~min} ; \mathbf{4 h}, 25.0$ $\min )$.
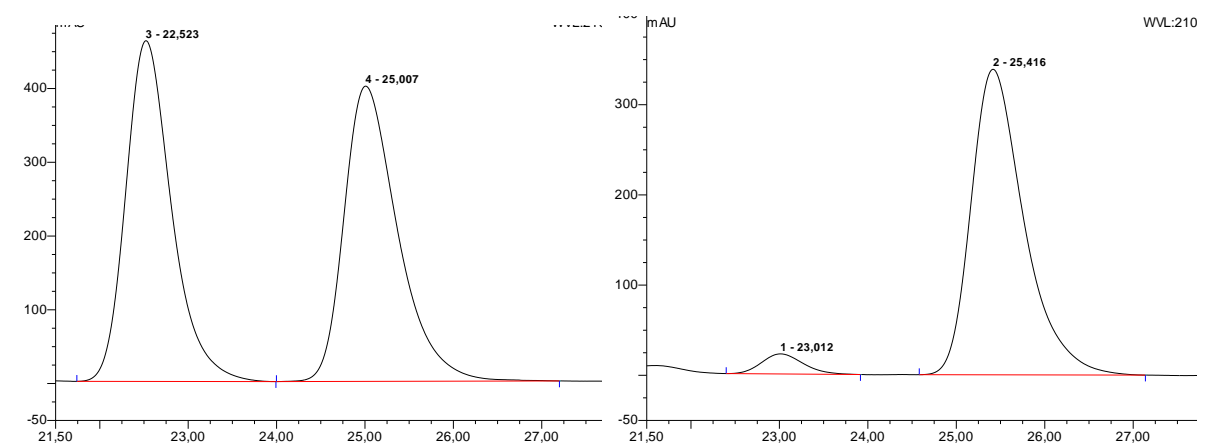

\begin{tabular}{|c|cc|cc|}
\hline & Area & Rel. Area $(\%)$ & Area & Rel. Area $(\%)$ \\
\hline$t_{\mathrm{R}}=22.5 \mathrm{~min}$ & 278.239 & 49.72 & 12.102 & 4.89 \\
\hline$t_{\mathrm{R}}=25.0 \mathrm{~min}$ & 281.343 & 50.28 & 235.231 & 95.11 \\
\hline
\end{tabular}




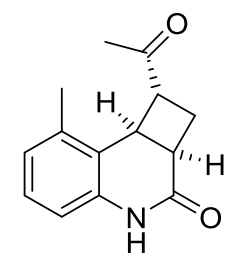

$\left.\mathbf{G C}\left[60{ }^{\circ} \mathrm{C}(1 \mathrm{~min}), 15{ }^{\circ} \mathrm{C} / \mathrm{min} \rightarrow 200{ }^{\circ} \mathrm{C}(30 \mathrm{~min})\right]=e n t-4 \mathbf{i}, t_{\mathrm{R}}=34.1 \mathrm{~min} ; \mathbf{4 i}, 34.8 \mathrm{~min}\right)$.
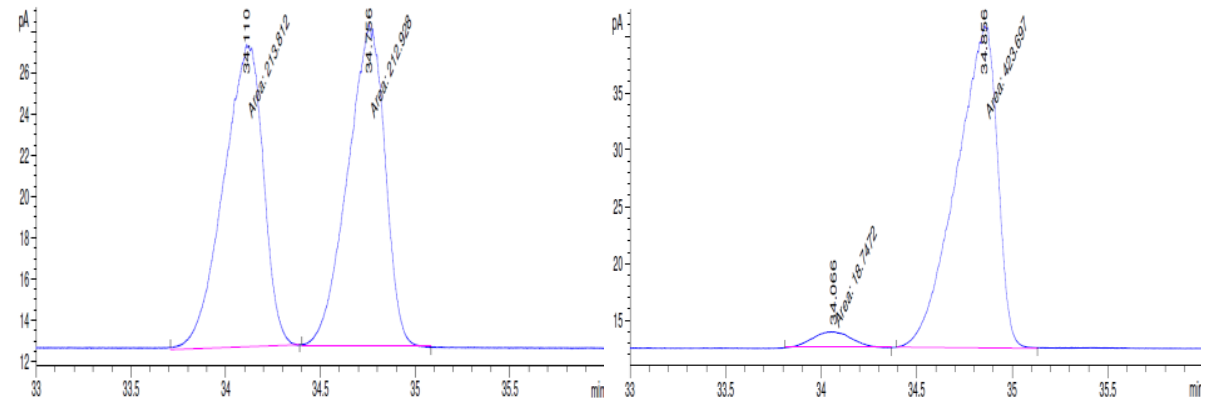

\begin{tabular}{|c|cc|cc|}
\hline & Area & Rel. Area $(\%)$ & Area & Rel. Area $(\%)$ \\
\hline$t_{\mathrm{R}}=34.1 \mathrm{~min}$ & 213.812 & 50.10 & 18.747 & 4.24 \\
\hline$t_{\mathrm{R}}=34.8 \mathrm{~min}$ & 212.928 & 49.90 & 423.697 & 95.76 \\
\hline
\end{tabular}<smiles>CC(=O)[C@H]1C[C@H]2C(=O)Nc3ccc(Br)cc3[C@H]12</smiles>

HPLC $(A D-H, 250 \times 4.6, n$-hexane/iso-propanol $=90: 10)=4 \mathbf{j}, t_{\mathrm{R}}=17.7 \mathrm{~min} ;$ ent-4j , $21.3 \mathrm{~min})$.
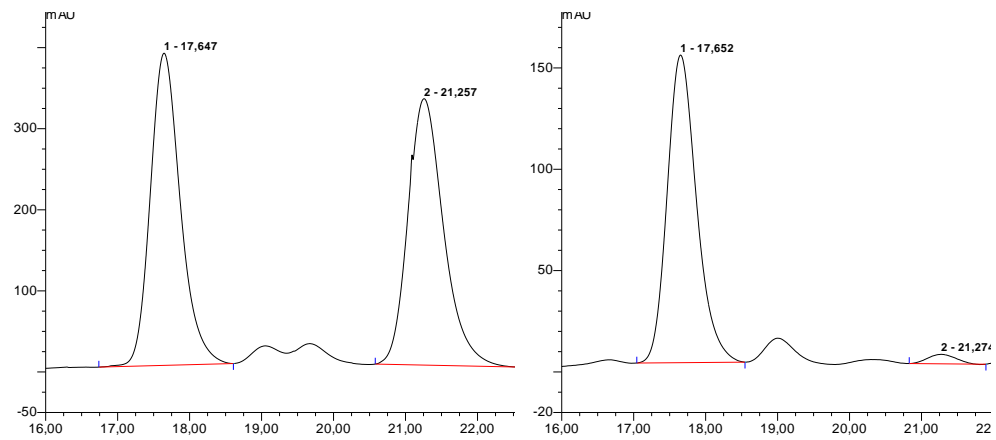

\begin{tabular}{|c|cc|cc|}
\hline & Area & Rel. Area $(\%)$ & Area & Rel. Area $(\%)$ \\
\hline$t_{\mathrm{R}}=17.7 \mathrm{~min}$ & 186.487 & 49.69 & 71.836 & 97.16 \\
\hline$t_{\mathrm{R}}=21.3 \mathrm{~min}$ & 188.783 & 50.31 & 2.099 & 2.84 \\
\hline
\end{tabular}




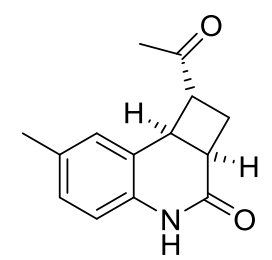

HPLC (AD-H, 250×4.6, $n$-hexane/iso-propanol $=95: 5)=4 \mathbf{k}, t_{\mathrm{R}}=35.7 \mathrm{~min} ;$ ent-4k, $44.3 \mathrm{~min})$.
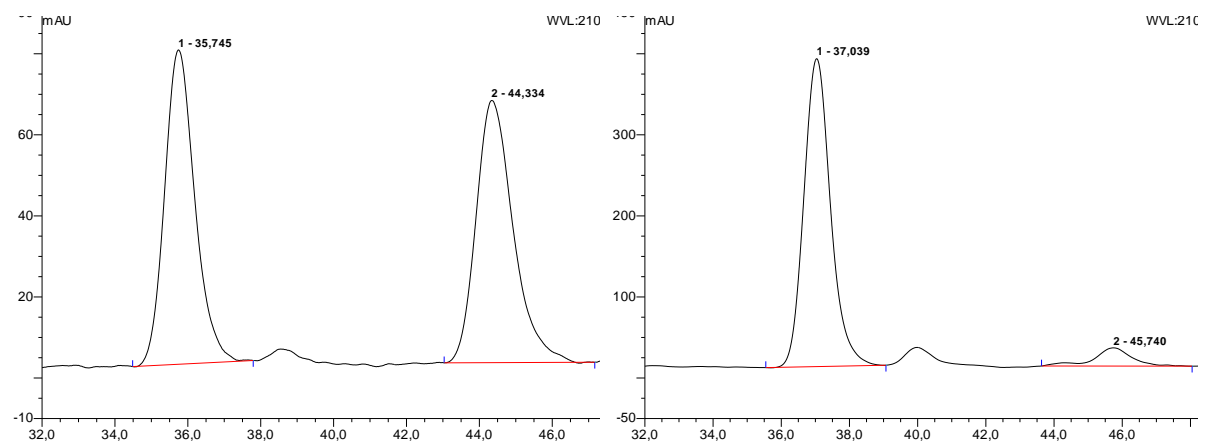

\begin{tabular}{|c|cc|cc|}
\hline & Area & Rel. Area $(\%)$ & Area & Rel. Area $(\%)$ \\
\hline$t_{\mathrm{R}}=35.7 \mathrm{~min}$ & 74.674 & 49.46 & 344.454 & 92.26 \\
\hline$t_{\mathrm{R}}=44.3 \mathrm{~min}$ & 76.317 & 50.54 & 28.908 & 7.74 \\
\hline
\end{tabular}

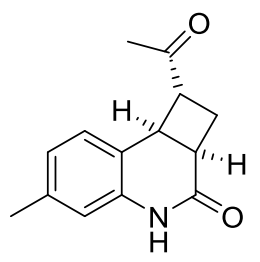

HPLC (AD-H, 250×4.6, $n$-hexane/iso-propanol = 90:10) = 4l, $t_{\mathrm{R}}=16.4$ min; ent-4l, $21.2 \mathrm{~min})$.
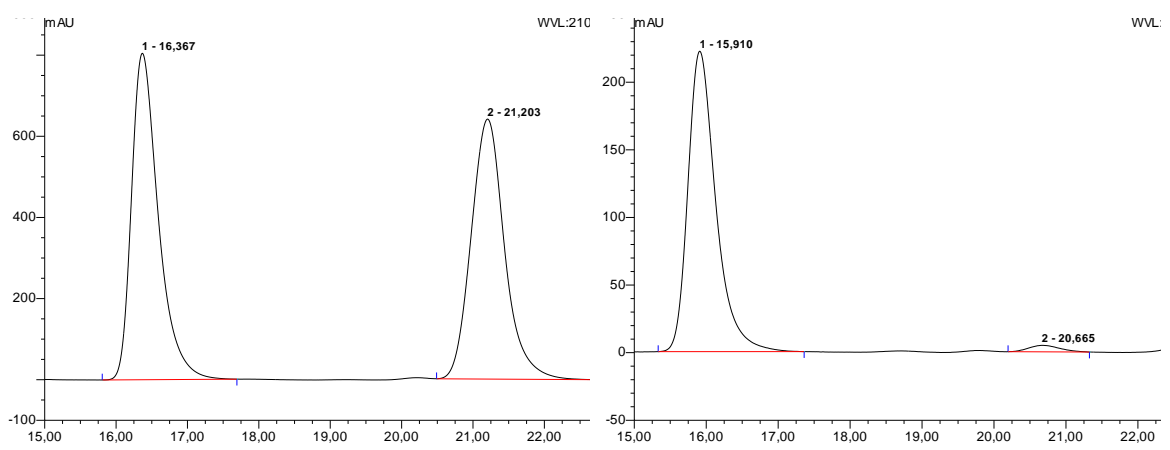

\begin{tabular}{|c|cc|cc|}
\hline & Area & Rel. Area $(\%)$ & Area & Rel. Area $(\%)$ \\
\hline$t_{\mathrm{R}}=16.4 \mathrm{~min}$ & 351.037 & 50.28 & 99.933 & 97.68 \\
\hline$t_{\mathrm{R}}=21.2 \mathrm{~min}$ & 347.185 & 49.72 & 2.377 & 2.32 \\
\hline
\end{tabular}




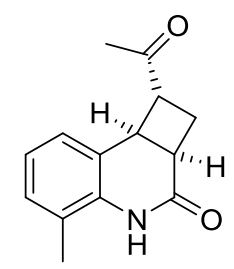

HPLC (AS-H, 250×4.6, $n$-hexane/iso-propanol $=70: 30)=\mathbf{4 m}, t_{\mathrm{R}}=11.6 \mathrm{~min}$; ent-4m, $14.7 \mathrm{~min})$.
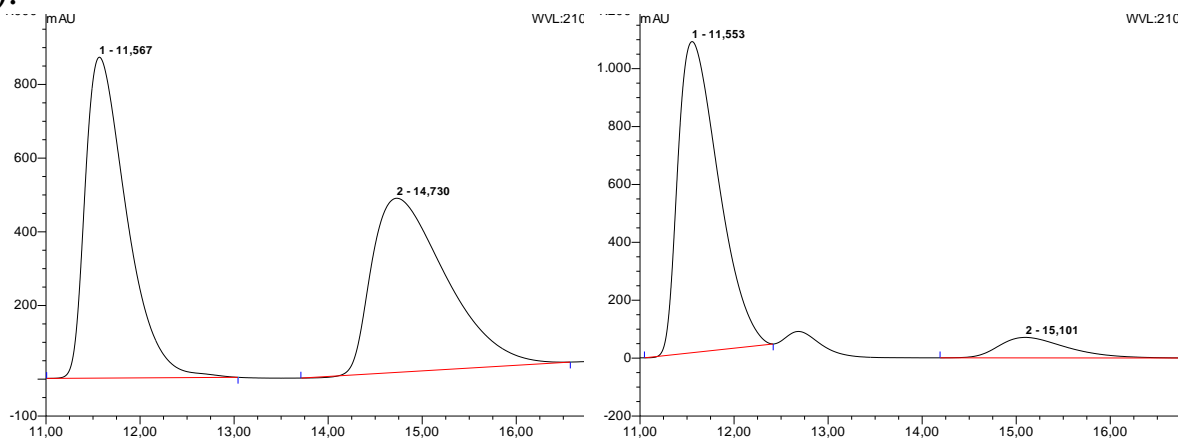

\begin{tabular}{|c|cc|cc|}
\hline & Area & Rel. Area $(\%)$ & Area & Rel. Area $(\%)$ \\
\hline$t_{\mathrm{R}}=11.6 \mathrm{~min}$ & 444.509 & 50.67 & 538.649 & 90.07 \\
\hline$t_{\mathrm{R}}=14.7 \mathrm{~min}$ & 432.785 & 49.33 & 59.403 & 9.93 \\
\hline
\end{tabular}

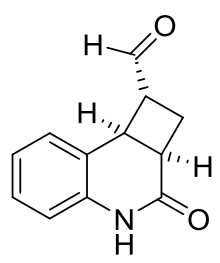

HPLC $(\mathrm{OJ}-\mathrm{H}, 250 \times 4.6, n$-hexane/iso-propanol $=90: 10)=e n t-\mathbf{4 n}, t_{\mathrm{R}}=19.4 \mathrm{~min} ; \mathbf{4 n}$, $22.6 \mathrm{~min})$.
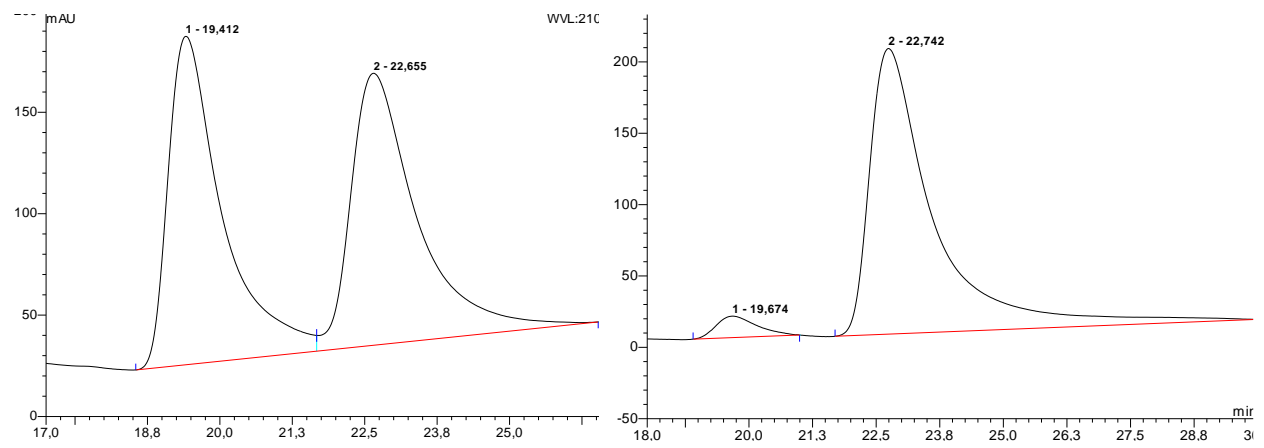

\begin{tabular}{|c|cc|cc|}
\hline & Area & Rel. Area $(\%)$ & Area & Rel. Area $(\%)$ \\
\hline$t_{\mathrm{R}}=19.4 \mathrm{~min}$ & 174.665 & 49.57 & 13.879 & 4.52 \\
\hline$t_{\mathrm{R}}=22.6 \mathrm{~min}$ & 177.728 & 50.43 & 293.261 & 95.48 \\
\hline
\end{tabular}




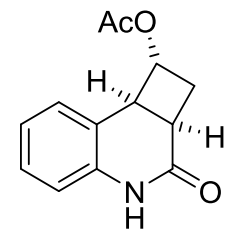

HPLC $($ AD-H, 250×4.6, $n$-hexane/iso-propanol $=90: 10)=40, t_{\mathrm{R}}=16.1 \mathrm{~min} ;$ ent-4o, $30.6 \mathrm{~min})$.
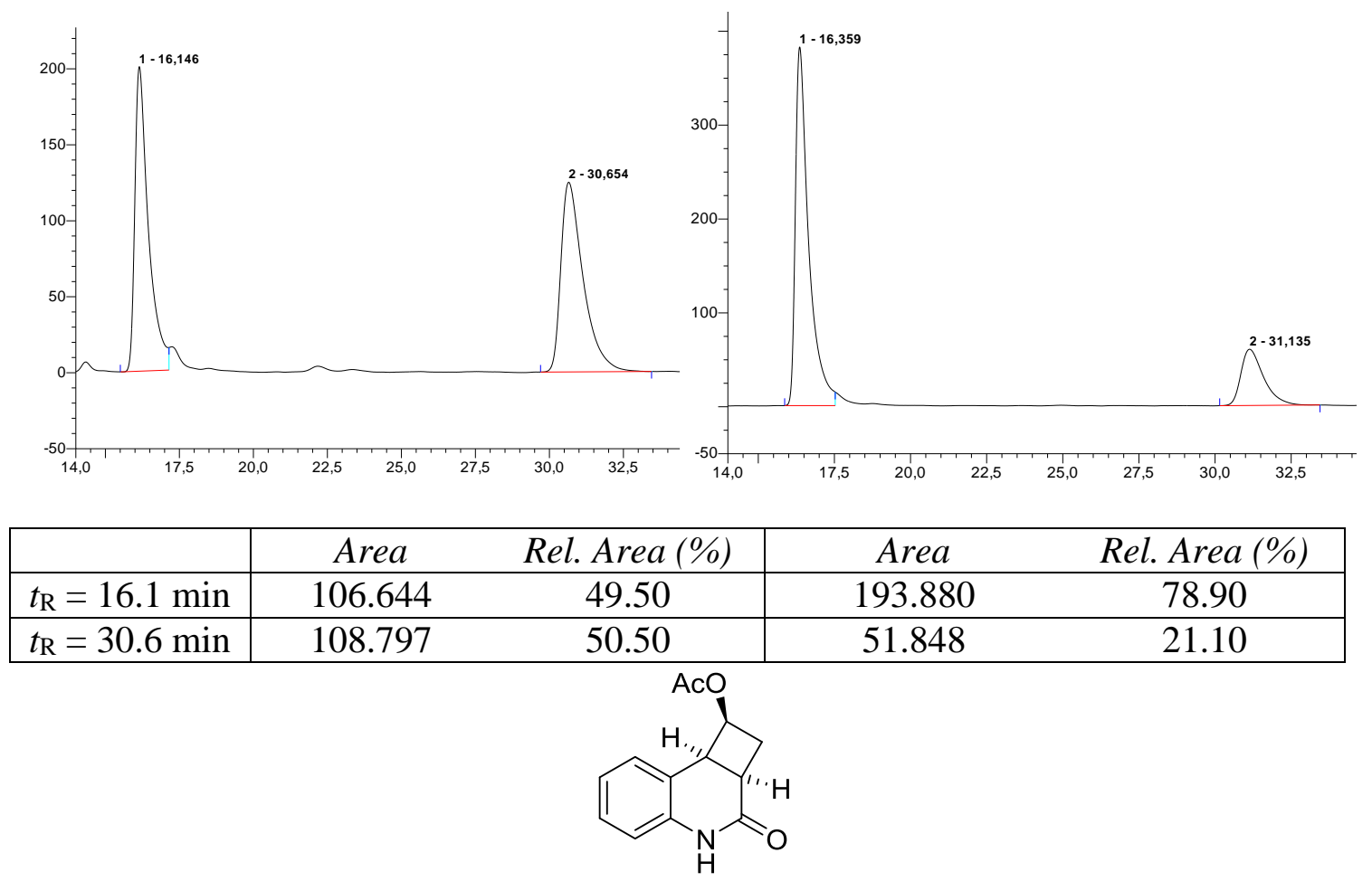

HPLC $\left(\right.$ AD-H, 250×4.6, $n$-hexane/iso-propanol = 95:5) $=\mathbf{S 1 7}, t_{\mathrm{R}}=33.9 \mathrm{~min} ;$ ent-S17, $36.2 \mathrm{~min})$.
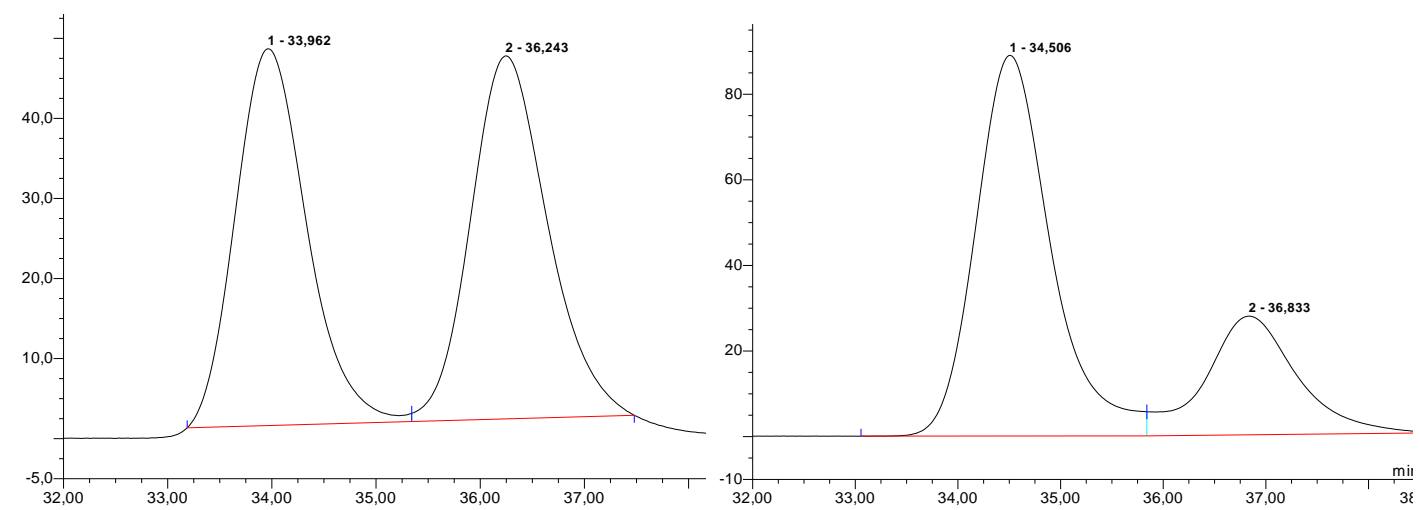

\begin{tabular}{|c|cc|cc|}
\hline & Area & Rel. Area $(\%)$ & Area & Rel. Area $(\%)$ \\
\hline$t_{\mathrm{R}}=33.9 \mathrm{~min}$ & 37.439 & 49.02 & 75.597 & 72.52 \\
\hline$t_{\mathrm{R}}=36.2 \mathrm{~min}$ & 38.942 & 50.98 & 28.651 & 27.48 \\
\hline
\end{tabular}




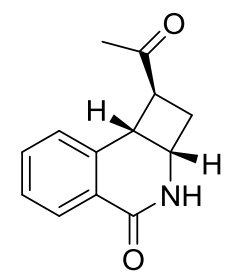

HPLC (AD-H, 250×4.6, $n$-hexane/iso-propanol = 90:10) $=$ ent-8, $t_{\mathrm{R}}=18.9 \mathrm{~min} ; \mathbf{8}, 29.4$ $\min )$.
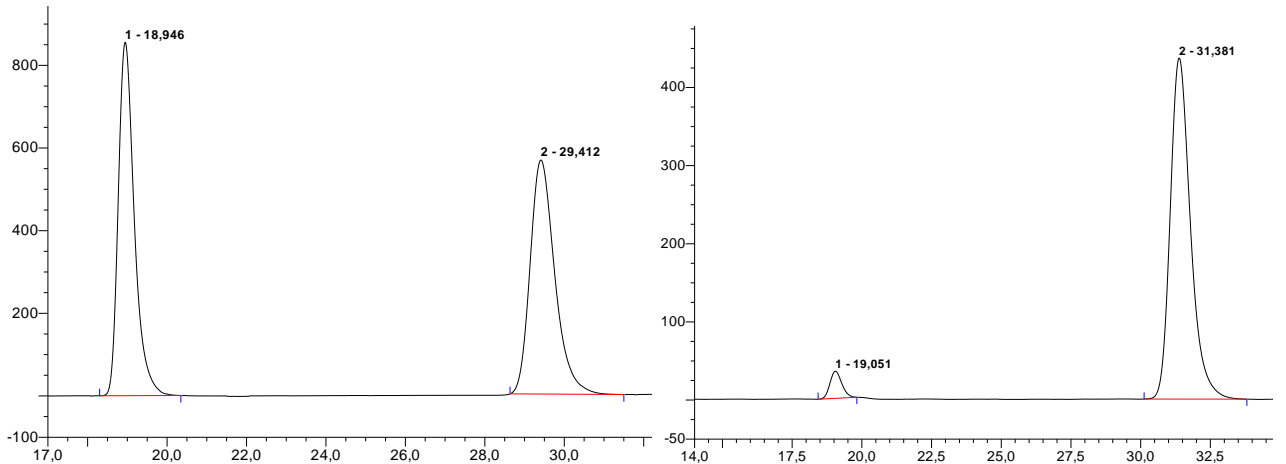

\begin{tabular}{|c|cc|cc|}
\hline & Area & Rel. Area $(\%)$ & Area & Rel. Area $(\%)$ \\
\hline$t_{\mathrm{R}}=18.9 \mathrm{~min}$ & 400.859 & 49.61 & 17.156 & 4.46 \\
\hline$t_{\mathrm{R}}=29.4 \mathrm{~min}$ & 407.086 & 50.39 & 367.140 & 95.54 \\
\hline
\end{tabular}

\section{HPLC traces of the solar irradiation experiment}

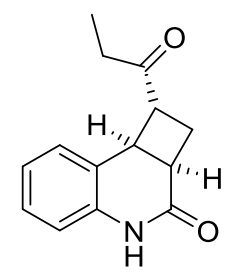

HPLC $($ AD-H, 250×4.6, $n$-hexane/iso-propanol $=90: 10)=\mathbf{4 f}, t_{\mathrm{R}}=15.4$ min; ent-4f, $16.9 \mathrm{~min})$.
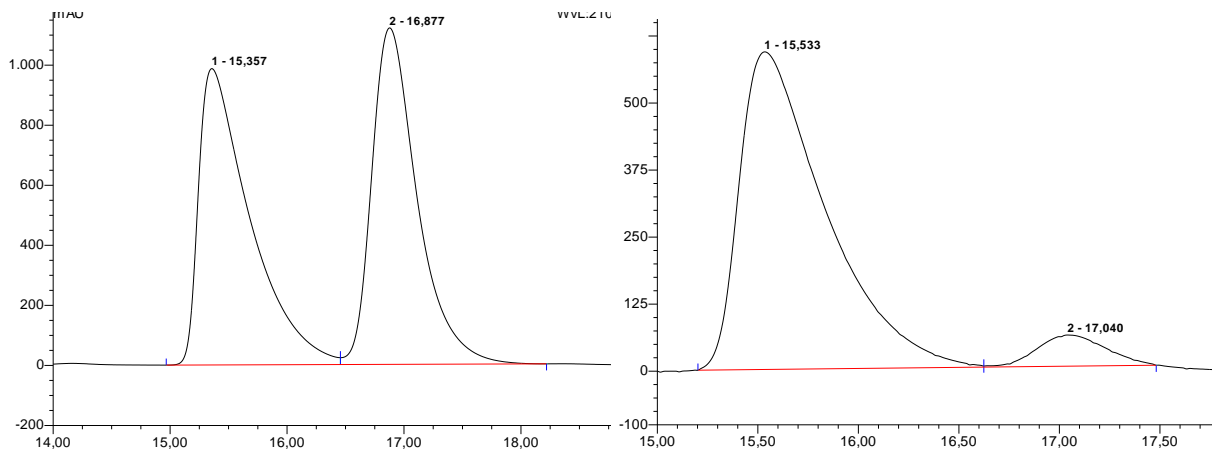

\begin{tabular}{|c|cc|cc|}
\hline & Area & Rel. Area $(\%)$ & Area & Rel. Area $(\%)$ \\
\hline$t_{\mathrm{R}}=15.4 \mathrm{~min}$ & 501.803 & 49.81 & 293.421 & 92.73 \\
\hline$t_{\mathrm{R}}=16.9 \mathrm{~min}$ & 505.639 & 50.19 & 23.013 & 7.27 \\
\hline
\end{tabular}

